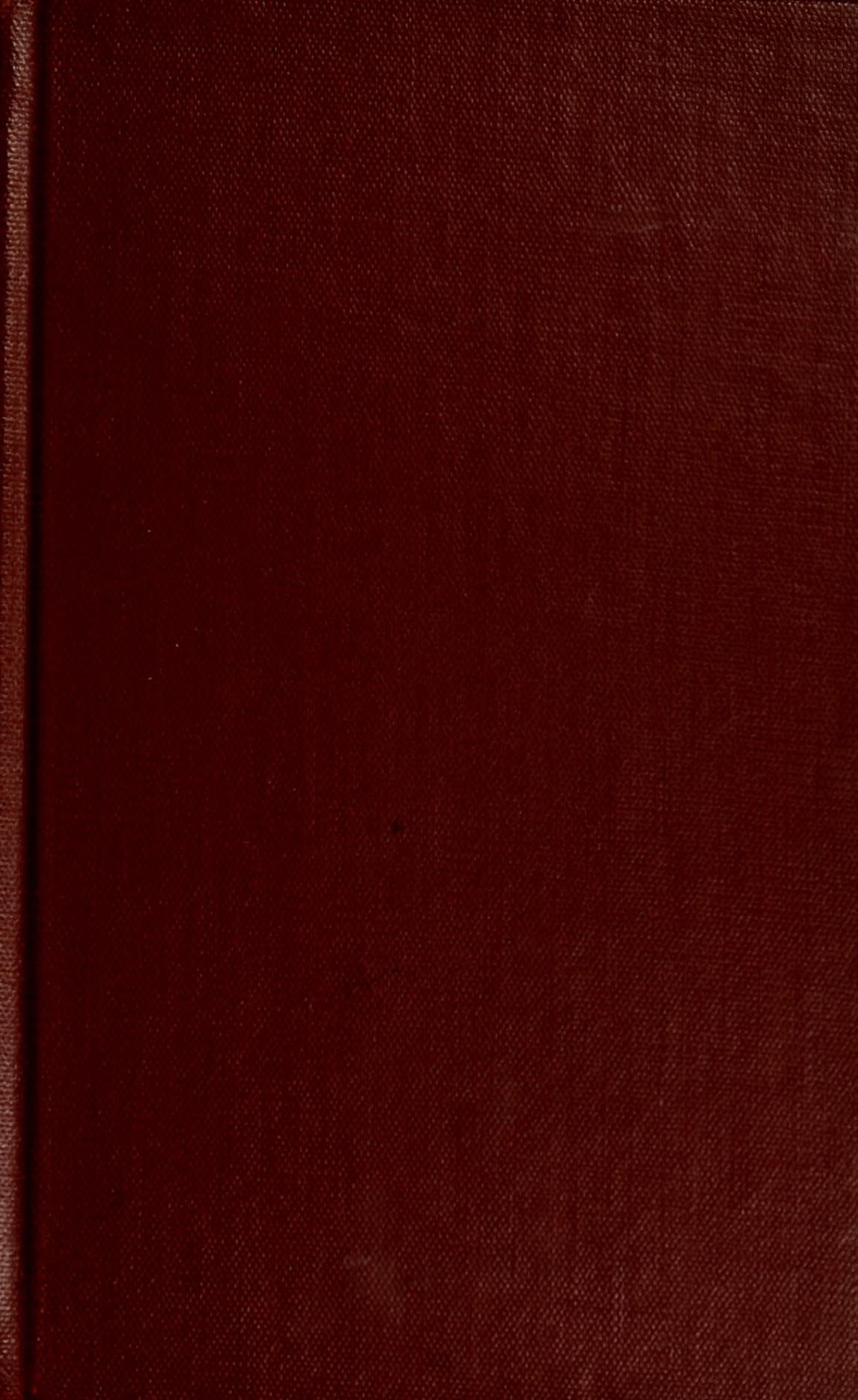




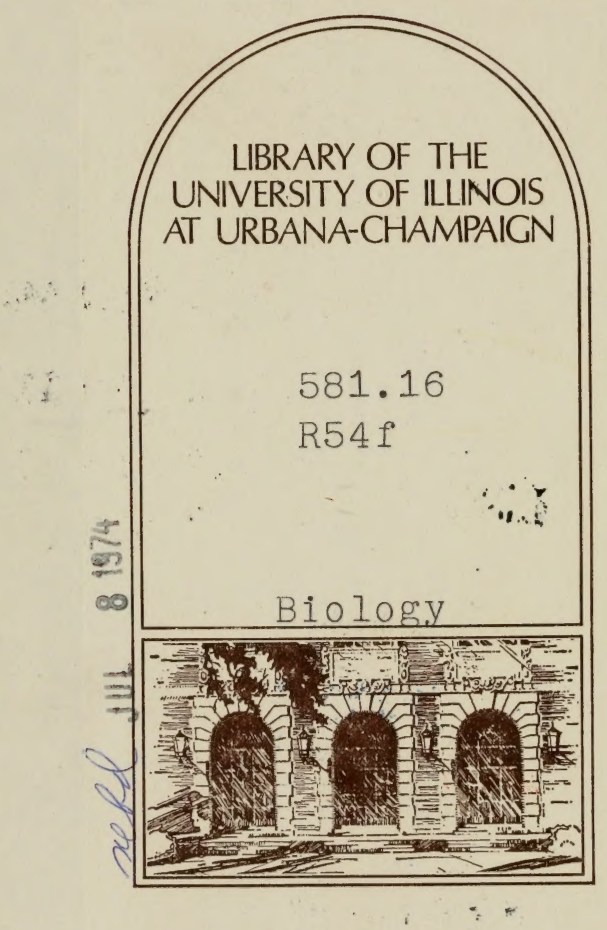


The person charging this material is responsible for its return to the library from which it was withdrawn on or before the Latest Date stamped below.

Theft, mutilation, and underlining of books are reasons for disciplinary action and may result in dismissal from the University.

To renew call Telephone Center, 333-8400

UNIVERSITY OF ILLINOIS LIBRARY AT URBANA.CHAMPAIGN

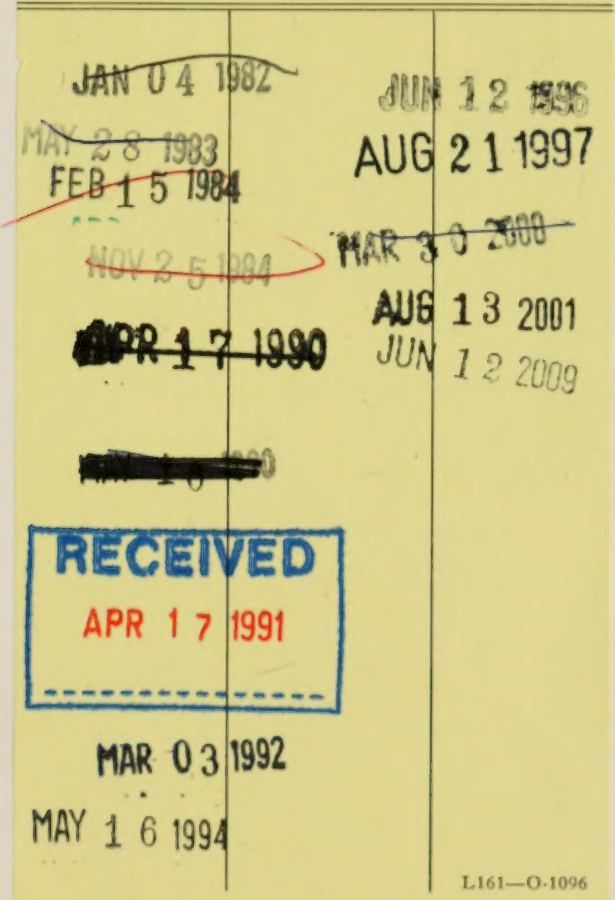



Digitized by the Internet Archive in 2011 with funding from University of Illinois Urbana-Champaign 



\title{
FLOWERS AND INSECTS
}

LISTS OF VISITORS OF FOUR HUNDRED AND FIFTY-THREE FLOWERS

\author{
By \\ CHARLES ROBERTSON
}


THE SCIENCE PRESS PRINTING COMPANY

LANCASTER, PA.

.

Copyrighted 1929

By Charles Robertson 


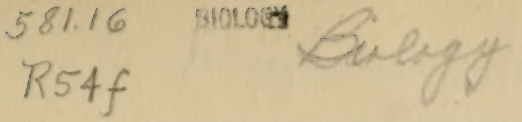

\section{PREFACE}

Beginning September, 1887, and ending July, 1899, the papers mentioned in the bibliography record 7,817 visits to 278 flowers. The present work records 15,172 visits to 441 flowers, nearly twice as many, excluding visits to 12 wind flowers. Mueller's "Fertilisation of flowers" records 5,231 visits, and his "Alpenblumen" gives 8,491 .

The observations were made within ten miles of Carlinville. The number of species of bees found on flowers at Carlinville is 296: compared with New York, 189; Connecticut, 231; New Jersey, 250. The list of visitors contains types of 232 new species of insects.

In identifying insects assistance has been afforded in Hymenoptera by W. H. Ashmead, Nathan Banks, J. C. Crawford, E. T. Cresson, R. A. Cushman, A. B. Gahan, W. J. Fox, L. O. Howard, Theo Pergande, and S. A. Rohwer; in Diptera by J. M. Aldrich, D. W. Coquillett, C. W. Johnson, J. R. Malloch, R. R. Parker, C. H. T. Townsend, and S. W. Williston; in Lepidoptera by G. H. French and C. A. Hart; in Coleoptera by C. A. Frost, C. A. Hart, Charles Liebeck, and Samuel Henshaw; in Hemiptera by C. A. Hart, H. M. Parshley, and P. R. Uhler; in Neuroptera by Nathan Banks and J. C. Crawford.

In 1887 names were rather readily obtained for Syrphidae, Bombyliidae, and Conopidae. In 1888 for 384 specimens of Diptera, estimated as 89 species, but certainly containing many more, only 17 specific determinations were received. It was hard to get names for Tachinidae until 1891-2 when Townsend supplied them. By Coquillett the Empididae were identified in 1894-5, and many Tachinidae and other Diptera in 1896-1901. For Sarcophagidae only a few names, some erroneous, were obtained until 1920 when they were determined by Parker.

In 1892-1901 many non-aculeate Hymenoptera were identified by Ashmead. Many manuseript names were supplied by him. Some of these have never been described, while others have been described under other names.

Particular difficulty was experienced in the ease of the bees, by far the most important for the investigation. Of the bees recognized only about 39.8 per cent are mentioned in Cresson's eatalogue of 1887 , and of those only about 46.6 per cent were identified for both sexes. At present I know both sexes in 81.7 per cent of the local bees. 
Generally, determinations of bees are unreliable except when both sexes of all of the species are known. When only one sex is known the other is probably mixed with that of another species. Formerly in Agapostemon four species had been distinguished in the females but the males were referred to another species, A. tricolor. There was little chance of separating another real species and this condition had the advantage of indicating five species instead of four. Sometimes the species are separated only in the males and the females ignored. Determinations of the females are twice as important as those of the males since they make twice as many flower visits.

On account of the impossibility of getting all of the bees identified it was necessary to describe the new species and match the sexes, thus making a digression which interrupted the study and involved an intrusion into a field which there was no inclination to cultivate.

Of all bees the bumblebees are the easiest to collect and identify. Eight species occur at Carlinville. Two species, Bombus americanorum and Bombias auricomus, had the females mixed under the name of Bombus pennsylvanicus and were referred to the male of Bombias auricomus, while the male of Bombus americanorum was referred to another genus as Apathus? elatus. The female of another species was identified for me as Bombus ridingsii. The worker was mentioned in 13, 579, 1891, and the male in 4, 65, 1891. But Bombus ridingsii turned out to be a sex name of Bombus bimaculatus, the prior name of the male of the same species.

From my experience I think that a specialist is competent to make determinations, or compare types, only at the time when he is making a careful study of a particular group. In 32 cases I received two different specific names for the same insect.

Your species monger describes the obvious and easy cases, the males and females as distinct species, and passes on the difficulties to some one else. North America is a large region and entomology, or even an order of insects, is a large subject. A specialist's reliability bears an inverse relation to the size of the region and the extent of the subject in which he affects authority. Students of bees describe species from all over North America, and even farther, but any one of them, and all of them together, can not identify both sexes of the bees of any locality.

All of the observations given here were made for the purpose of ascertaining the different kinds of insect visitors. Collectors' notes are worthless as data of anthecology because the collector is looking out for particular kinds of insects and not for all of the kinds which 
occur on the flowers. They are worthles as data of inseret habits berause the collector is eertain to seleet the flowers which are most favorable for observation and which are most aftrative to the kinds of insects he is after. Collectors notes almost invariably fail to indieate what the inserts are doing on the flowers and whether they affect pollination or not.

The following peroms made additions to the lomal lists of the plants mentioned-Prof. I. II. Benham: Blephilin hirsuln, Evupatorium perfoliatum. Teucrium canadense: George T. Palmer: Erigeron ramosus, Stachys pulustris; Roherton Palmer: lallisin nyctelan, Myporis hirsutn. Polemonium reptans, Sambucus canndensis, Scutellaria perrula: H. B. Parks: Achillon millefolium, Apocynum cannabinum, Blophilin cilintn. Cassin chamnarrista, Cianothus americunus, Cirula maculaln, C'ucurbita pepo, Eryngium yuccifolium, Eulophus amricanus, Lepachys pinnata. Monarda fistulosa, Pastinara saliza, Polygala sanguinea, Prunus amerieana, P. serotina, Pycnunthemum flexusum, P. pilosum, Rhus copallina, R. glabra, Rosa humilis, R. sotigera, Salix nigra, Sambucus canadensis, Stenanthium angustifolium, Trronica virginica.

In some eases the lists were based on the older nomenelatnre, so that I did not eare to rowrite and rearrange them. In the preliminary list some of the later sperifu names are given in parenthesis. After ehanging Danais archippus to Anosia plexippus it is a little tiresome to change it back to former name, and then to Danaus. Anyhow, biologiral nomenelatare is synonymical, not binomial. The older names usually have more important relation to antherologieal literature. Authors will have to use them whether they like them or not. Often the strnonymy is a subject of controversy. Of 455 plants mentioned here, about 70 have their names ehanged in Gray's Manual. 


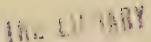

$$
\begin{aligned}
& \text { of उHE }
\end{aligned}
$$

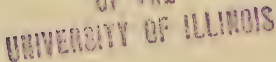




\title{
Flowers and Insects
}

\author{
Signs, Abbrevlations, Exc.
}

$a b=$ abundant $; e=$ collecting pollen; $e f=$ collecting and feerling on pollen; $(\mathrm{sp}=$ collecting stray pollen; eult = eultivated; expl $=\mathrm{ex}$ ploratury visit; $f=$ feeding on pollen; $f q=f$ requent ; $f$ s $=$ feeding on st ray pollen; $g n=$ gnawing; in eop $=$ in copula ; int = int roduced, not indigenous; $I_{p}=$ labial $p a l p s ; M_{a}=$ non-social long-tongued bee flower; Mas = social long-tongued bee flower; $\mathrm{Mi}=$ non-social shorttongued bee flower; Mis=social short-tongued bee flower; $m p=$ maxillary palps; $\mathrm{n}=$ non-pollinating; $\mathrm{O}=$ ornithophilous; $\mathrm{ol}=\mathrm{oli}$ golege; $P$ ol = polytropie $;$ pred = predaceous $; R=$ red and all dark eolors; $\mathrm{S}=$ sphingophilous; $\mathrm{se}=$ sucking nectar and collecting pollen; $\mathrm{si}=$ sucking nectar and feeding on pollen; $\mathrm{W}=$ white; $\mathrm{Y}=$ yellow, greenish to orange $; \hat{\sigma}=$ male $; q=$ female $; \not=$ worker.

When no other indications are used after a name, the iuseet is counted as sucking nectar legitimately and effecting pollination. Dates conneeted by a dash after "visitors observed" do not indicate the number of days, but only the period within which the observations were made. Signs in parentheses after a group name mean that the signs belong to each one of the insects mentioned in the group. For example, "Malict. ( $q$, e )" means that all of the following IIalictidae are females and all collecting pollen.

After family names the ending "idae" is not repeated, as If alict. for IIalictidae. When only one species of a genus was observed, the specific name is not repeated in the lists. Thus $A$ pis = A pis mellifera $\Varangle$. This aroids repeating mellifera 216 times. Four hundred and forty-four genera have only one species each. which it is not necessary to repeat.

The plant mames used are those of the Tth edition of Gray's Manual of Botany, 1908.

Numbers in parentheses, for example (12: 459), refer to the titles and pages in the bibliography, or to speeies and individuals.

\section{General Srnonymical List}

The following is a list of all the inserts taken on flowers. It eontains authors' names which are given here once for all and are not repeated in the special lists.

Names which occur often enough to justify it are abbreviated as follows: $-\mathrm{Al}=$ Aldrich, Ash. $=$ Ashmead. B. B. $=$ Brauer and Bergenstamm, B. L. = Boisdusal and Jeconte, $\mathrm{Br}=$ Brulle, $\mathrm{Bs} .=$ 
Boisduval, Bv. $=$ Beauvois, Cc. $=$ Cockerell, Cm. $=$ Cramer, Cq. $=$ Coquillett, Cr. $=$ Cresson, Dh. $=$ Dahlbom, Dg. $=$ De Geer, Dr. $=$ Drury, Ds. $=$ Desvoidy, Ed. $=$ Edwards, F. $=$ Fabricius, Fl. $=$ Fallen, Fr. = Forster, Gn. = Guenee, Gr. = Germar, Grv. = Gravenhorst, Hb. = Hübner, Hr. $=$ Herbst,$H . \quad$ S. $=$ Herrich Schaeffer,$L=$ Linnaeus, Lc. $=$ Le Conte, Lp. $=$ Lepeletier, Lw. = Loew, Mc. = Maequart, Mg. = Meigen, MI. $=$ Melsheimer, Nr. $=$ Norton, Nw. = Newman, Ol. $=$ Olivier, O. S. $=$ Osten Sacken, Pc. $=$ Packard, Pr. $=$ Provancher, Pt. $=$ Patten, Rb. = Robertson, Rl. = Riley, Sc. $=$ Scudder, Sm. $=$ Smith, Ss. $=$ Saussure, Tn. $=$ Townsend, $\mathrm{Wd} .=$ Wiedemann, Wk. $=$ Walker, Wl. $=$ Williston .

\section{BIRDS}

Trochilidae: Trochilus colubris L.

\section{LONG-TONGUED BEes.}

Anthophoridae: Amegilla walshii Cr., Anthemoessa abrupta Say, Anthophora ursina Cr., Clisodon terminalis Cr., Emphoropsis floridana Sm.; Apidae: Apis mellifera L.; Bombidae: Bombias auricomus Rb., type, scutellaris Cr., separatus Cr., Bombus americanorum F., consimilis Cr. (vagans Sm.), pennsylvanicus Dg., ridingsii Cr., (bimaculatus Cr.), impatiens Harris, Psithyrus laboriosus F., variabilis Cr.; Ceratinidae (females mixed); Ceratina dupla Say, Zadontomerus calcaratus Rb., type; Emphoridae: Emphor bombiformis Cr., Melitoma taurea Say; Epeolidae: Argyroselenis minimus Rb., type, Epeolus autumnalis Rb., type, bifasciatus $\mathrm{Cr}$., coreopsis $\mathrm{Rb}$. MS, interruptus $\mathrm{Rb}$., type, lectoides Rb., type, pusillus Cr., Triepeolus concavus Cr., concolor Rb., type, cressonii Rb., type, donatus Sm., helianthi Rb., type, lunatus Say, micropygius $\mathrm{Rb}$., type, nevadensis $\mathrm{Cr}$., pectoralis $\mathrm{Rb}$., type, remigatus F., simplex Rb., type; Euceridae: Anthedon compta $\mathrm{Cr}$., Cemolobus ipomoeae Rb., type, Epimelissodes atripes Cr., illinoensis Rb., type, obliqua Say, Florilegus condignus Cr., Melissodes agilis $\mathrm{Cr}$, asteris $\mathrm{Rb}$., type, autumnalis $\mathrm{Rb}$., type, bimaculata $\mathrm{Lp}$, boltoniae Rb., type, enici Rb., type, coloradensis $\mathrm{Cr}$., comptoides $\mathrm{Rb}$., type, coreopsis $\mathrm{Rb}$., type, nivea $\mathrm{Rb}$., type, pallida Rb., type, petalostemonis $\mathrm{Rb}$., type, simillima $\mathrm{Rb}$. , type, trinodis $\mathrm{Rb}$. , type, variabilis $\mathrm{Rb}$., type, vernoniana $\mathrm{Rb}$., type, vernoniae $\mathrm{Rb}$., type, Peponapis pruinosa Say, Tetralonia belfragei Cr., dilecta $\mathrm{Cr}$. (speciosa?), dubitata $\mathrm{Cr}$., illinoensis $\mathrm{Rb}$., type, robertsoni Cc., type, rosae Rb., type, Xenoglossa strenua Cr., Xenoglossodes albata Cr.; Megachilidae: Osmiinae, Osmiini: Alcidamea simplex Cr., truncata Cr., Ashmeadiella bucconis Say, Centrosmia bucephala Cr., Ceratosmia lignaria Say, Diceratosmia conjuncta Cr., Gnathosmia 
georgiea Cr., Hoplitis eylindrieus Cr., Leneosmia alhiventris Cr.. Monilosmia eanadensis Cr., Osmia atriventris C'r., collinsiae Rh.. type, cordata Rb., type, distineta Or., illinoensis Rh., type. pumila Cr.; Trypetini: Neotrypetes barbatus lib., type, productus Rib., type (9 mixed with that of the next), trumcatus Rh. MS, Prochelontoma philadelphi Rh., type: Megathilinate, Megachilini: Anthemois emtuneularis L., Chelostemoides rufimanus Rb., type, Cyphupgoa monlivaga Cr., Mrearhile addenda Cr., hrevis Say, generoma Cr., mendica Cr., petulans Cr., G-dentata Kb., typ., strophostylis Rh., type, Oligotropus campanulae Rh., type, Saromster georgira C'r., Sayajis pulliearis Say, fugnata Siay, sayi Gr.. Xanthosarus latimnne Say; Coelioxyini: Corelioxys alternata Say, grrmana C'r., modesta Sm., S-rlentata Say, ruftarsis Sm., sayi Rb.. type; Melectides: Melecta interrupta Cr., Dombomelereta thoraciea Cr.: Nomadidae: Centrias ameriranus Kirby, erigerontis Rh), lype, Cephen texanus Cr., Gnathias enneatus lib., type, ovatus Rb., type, xamthoparius Rb. MS, Heminomala obliterafa Cr., Holonomada aftabilis Cr.. plaeida Cr., superba Cr., vineta Say, Nomada eresonii lib., lype, denticulata Rb., type, illinoensis Rb., lype, parra Rh., lype, salieis Rb., tyye, sayi Rh., type, simplex Rh., type, vieima Cr.. Phor integer Rh., type, Xantbidium dentariae Rh., type, luteoloiles Rb., ty/u, luteolum OL.; Pusitidue: Holeopasites heliopsis Rh., type, illinoensis Rh., type: Stelididae: Anthidinae, Anthidini: Inthidium coenatum Cr., psoraleate Rb., tyfue, Dianthidium boreale Rb. tylue; Stelidini: Chelynia foederalis Sm., Microstelis lateralis Cr., Stelidium trypetinum lRb., type : Xolocopidat: Xylocopa virginica Dr.

\section{Siort-TONGUED BEES.}

Andrenidas: Andrena arabis Rh., type, earlini ('e. typu, romi Rh., type, dumningii ('e., erythrogastra Ash., erythronii Rh., ty/u, gemaii Rh., type, illinorensis Rh.. Iy/u, macoupinensis Rh., type, mamlibularis Rb.. type, nasonii Rb., type, nierae Rb., type, nothescorrli Rh., type, nulurula Sm., panieulatae Rh., MS, jatyparia Rb. (integra Sm.), pruni Rb., fype, salicacea Rb., type, salieis Rh., type. salietaria Rb., tyme, sayi Rb.. type, tridens Rb., tyme, Iomelissa violae Rh., type. Opandrena eressonii Rh., type, dubia Rh., typo. nisnrabilis Cr., personata Rh., tyle, serotina Rb., typt, zizine Rh.. type. Parandrena andrenoides Cr., Pterandrena aliciae Rb., ly asteris Rb., type, helianthi Rh., ty/u, Lrigiana Rib., typt, laumeea IRb., type, pul.hella Rb., type, rudbeckias Rh.. type, solidagin is Rb., type. Ptilandrena erigeniae Rb., typm, g. masulati Rh., type, polemonii Rb., tyme. Trachandrena elaytoniae Rb. type, eratareci Rb..

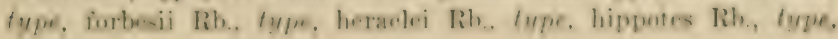


mariae Rb., type, nuda Rb., type, obscura Rb., type, quintilis Rb., type, rugosa $\mathrm{Rb}$., type, spiraeana $\mathrm{Rb}$., type; Colletidae: Colletes aestivalis $\mathrm{Pt}$., albescens Cr., americanus $\mathrm{Cr}$., armatus $\mathrm{Pt}$., brevicornis Rb., type, compactus Cr., eulophi Rb., type, inaequalis Say, latitarsis Rb., type, nudus Rb., type, productus Rb., type, robertsonii Dalla Torre, type, similis Rb., type, speciosus Rb., type, willistonii Rb., type; Dufoureidae: Halictoides marginatus Cr.; Halictidae: Agapostemon radiatus Say, splendens Lp., texanus Cr., viridulus F. (virescens F.), Augochlora fervida Sm., viridula Sm., Chloralictus albipennis Rb., type, caeruleus Rb., type, coreopsis Rb., type, cressonii $\mathrm{Rb}$., type, foveolatus $\mathrm{Rb}$., type, illinoensis $\mathrm{Rb}$., type, nymphaearum Rb., type, obscurus Rb., type, pilosus Sm., pruinosus $\mathrm{Rb}$., type, smilacinae Rb., type, sparsus $\mathrm{Rb}$., type, tegularis $\mathrm{Rb}$., type, testaceous Rb., type, versatus Rb., type, zephyrus Sm., Curtisapis coriacea Sm., forbesii Rb., type, fuscipennis Sm., Dialictus anomalus Rb., type, Evylaeus arcuatus Rb., type, foxii Rb., type, nelumbonis Rb., type, pectinatus Rb., type, pectoralis Sm., 4-maculatus Rb., type, truncatus Rb., type, Halictus lerouxii Lp., parallelus Say, Odontalictus ligatus Say, Oxystoglossa confusa Rb., type, pura Say, similis Rb., type, Paralictus eephalicus Rb., type, platyparius Rb., type, simplex Rb., type, Seladonia fasciata Nylander; Sphecodini: Dialonia antennariae Rb., type, Drepanium falciferum Pt., Machaeris illinoensis Rb., type, stygia Rb., type, Proteraner ranunculi Rb., type, Sphecodes arvensis Pt., clematidis Rb., type, heraclei Rb., type, minor Rb., type, Sphecodium cressonii Rb., type, pimpinellae Rb., type, pyenanthemi Rb., type, smilacinae Rb., type; Macropididae: Macropis steironematis Rb., type; Nomiidae: Paranomia nortonii Cr.; Panurgidae: Anthemurgus passiflorae Rb., type, Calliopsis andreniformis Sm., coloradensis Cr., Heterosarus parvus Rb., type, Perdita 8-maculata Say, Perditella boltoniae Rb., type, Pseudopanurgus albitarsis Cr., asteris Rb., type, compositarum $\mathrm{Rb}$., type, labrosiformis Rb., type, labrosus Rb., type, rudbeckiae Rb., type, rugosus Rb., type, solidaginis Rb., type, Verbenapis verbenae Cc., Zaperdita maura Ce.; Prosopididae: Prosopis crataegi Rb. MS, eulophi Rb., type, illinoensis Rb., type, nelumbonis Rb., type, pygmaea Cr., saniculae Rb., type, sayi Rb., type, thaspii Rb., type, ziziae Rb., type.

\section{Other Hymenoptera.}

\section{(367)}

Bembicidae: Bembix nubillipennis Cr., spinolae Lp., Bicyrtes 4-fasciata Say, ventralis Say, Megastizus brevipennis Walsh, Microbembex monodonta Say, Sphecius speciosus Dr., Stictia carolina F., Stictiella pictifrons Sm., Stizus unicinetus Say; Bethylidae: 
Goniozus cellularis Say; Braconidae (including Alysiidae and Vipionidae): Aenignostomus longipalpus Cr., Agathis areolata Ash., type, vulgaris Cr., Apanteles crassicornis $\mathrm{Pr}$., illinoensis Ash. MS, terminalis Gahan, Bassus sinctus Say, Braeon ashmeadi Morrisun, type, longieauta Pr., simplex Cr., trifolii Ash. (pyemaeus Pr.), vernoniae Ash. (mellitor Say), Cardiochiles tibiator Say, Chelomus serieeus Say, Darnusa flavieineta Ash., Earinus limitaris Say, Euphorus mellipes Cr., Inbrobraeon geleehiate Ash., Iphiaulax rugator Say, Marrodyetium apieatus Pr., Microbracon tortrieienla Ash. (variabilis Pr.), Mierodus simillimus Cr., Mirrogaster geleehiae Rl., Mieroplit is ceratomiae Rl., eroceipes Cr., gortynae Rl., Monogonogastra fuscipennis Ash. MS, Opius fuseipennis Gahan, rufieeps Pr. (provaneheri Dalla Torre), Orgilus rileyi Ash. (divisus $\mathrm{Cr}$.), Toxoneuron abdominale Cr., viator Say, Urosigalphus femoratus Ash., Vipio robertsonii Ash. IIS; Chalcididue (including Elasmidae, Eneyrtidae, Eulophidae, Eurytomidae, Leuenspidae, Perilampidae, Pteromalidae): Bruchophagus funebris Howard, Chaleis flavipes F. (ovata Say), Chrysocharis oseinidis Ash., Elasmus nigripes Howard, Eurytoma diastrophi Walsh, studiosa Say, Eutelus flavipes Wk., Eutrichosoma mirahilis Ash., Haltichella ornata Wk., xanticles Wk., Leueospis affinis Say, Pachyneuron mesograptae Ash., Perilampus eyaneus Br., fulvieornis Ash., hyalinus Say, platygaster Say, robertsoni Crawford, type, triangularis Say, Smicra debilis Say, torvina Cr., Syntomopus americanus Ash.; C'rysididue: Chrysis intricata Br., texana Gribodo, Gonnchrysis perpulchra Cr., Hedychridium dimidiatum Say, Mydychrum parvum Aaron, violaceum Br., wiltii Cr., Holochrysis hilaris Dh., verticalis Pt., Holopyga ventralis Say, Monochrysis parvula F., Notozus hyalinus Aaron, Tetrachrysis caerulans F., montana Aaron, nitidula F., nortonii Aaron, venusta Cr.; Crabronidae: Anacrabro oeellatus Pe., Blepharipus harringtonii Fox, Clytochrysus obeeurus Sm., Ectemnius atrieeps Cr., Entomognathus texanus Cr., Hypoerabro 10-maeulatus Say, Lindenius errans Fox, robertsoni Rohwer, type, Lophocrabro singularis Sm., Protothyreopus bigeminus Pt., rufifemur Pe., Pseudoerabro ehrysargirus Lp., Rhopalum pedieellatum Pe., scutellatum Say, Solenius interruptus Lp., Synothyreopus tumidus Pe., Xestocrabro 6-marulatus Say, texanus Cr., trifaseiatns Say; Eumenidat: Aneistrocerus campestris Ss., capra Ss., eatskillensis Ss., tigris Ss., unifasciatus S\&., Eumenes belfragri Cr., Praternus Say, Leionotus anormis Say, arvencis Ss., elypeatus Rb., type, dorsalis F., foraminatus Ss., fulvipes Se, fundatus Cr. histrionalis Ibb., type, illinoensis Rh., type, leueomelas Ss., megaema Lp., molestus Ss., perennis Ss., 4-sertus Say, scroplublaviae Rb. MS. 
vagus Ss., ziziae Rb. MS, Monobia 4-dens L., Pterochilus 5 -fasciatus Say, Symmorphus debilis Ss., walshianus Ss., Zendalia (MS) alcolhuus Ss., zendaloides Rb., type, Zethus spinipes Say; Evaniidae: Foenus incertus Cr., tarsatorius Say, Hyptia reticulata Say; Figitidae: Aspicera albihirta Ash., Cothonaspis stigmata Say, Eucoila mellipes Say, Eucoilidia canadensis Ash., Figites impatiens Say, 5-lineata Say, Psilodora erythropus Ash., impatiens Say, Trirhoptrasema ashmeadi Rohwer and Fagan, Xyalosema armata Say; Formicidae: Camponotus eastaneus Latr., herculanus L., Formica fusca L., rufibarbis F., schaufussi Mayr, Lasius niger L., Tapinoma sessile Say; Myrmicidae: Cremastogaster lineolata Say; Ichneumonidae: Agrothereutes limatus Cr., mundus Pr., Angitia obscura Cr., Bathycetes scutellaris Cr., Ceratosoma fasciata Cr. (ornata Say), Colpognathus helvus Cr., Cremastus epagoges Cushman, retiniae Cr., Cryptus exulans Cr., persimilis Cr., rufoannulatus Pr., Diplazon sycophanta Walsh, Eiphosoma texana Cr., Exetastes obscurus Cr., suaveolens Walsh, Exyston abdominalis Cr., Gambrus albonotatus Pr., apicatus Pr., Glypta rufiseutellaris Walsh, simplicipes Cr., Hemiteles gracilariae Ash., Ichneumon flavizonatus Cr., funestus Cr. (laetus Br.), jucundus Br., longulus Cr., succinctus Br., Idiolespa analis Grv. (limatus Cr.), Lampronota americana Cr., coxalis Ash. MS, varia Cr., Limneria eurycreontis Ash., fura Cr., Lissonota tricincta Ash. MS, Metopius polycinctorius Say, terminalis Ash. (seminiger Cr.), Ophion bifoveolatum Br., Pharsalia virginiensis Cr., Phygadeuon vulgaris Cr., Pimpla annulipes Br., marginata Pr., notanda Cr., novita Cr., Polyblastus citripes Ash. MS, Polysphincta slossonae Davis, Porizon albipennis Cr., conotracheli Rl., Proclitus mellipes Pr., Trogus vulpinus Grv., Tryphon affinis Cr.; Larridae: Astatus bicolor Say, unicolor Say, Larra analis F., Larropsis distineta Sm., Lyroda subita Say, Notogonidea argentata Bv., Tachysphex acutus Pt., belfragei Cr., mundus Fox, tarsatus Say, terminatus Sm., Tachytes aurulentus F., distinctus Sm., elongatus Cr., harpax Pt., maestus Mikel, mandibularis Pt., mergus Fox, obscurus Cr., pepticus Say, sericatus Cr.; Mutillidae $\left({ }^{\top}\right)$ : Mutilla hexagona Say, sayi Blake, scrupea Say, Pseudomethoca canadensis Blake, Sphaerophthalma macra Cr.; Myrmosidae: Myrmosa parvula Fox, type, unicolor Say; Nyssonidae: Alyson trianguliferus Pc., Hoplisoides nebulosus Pc., Hoplisus atricornis Pc., simillimus Sm., Nysson plagiatus Cr., rufiventris Cr., Zanysson aurinotus Say, plesia Rohwer, Pseudoplisus divisus Sm., phaleratus Say, Silaon inerme Cr.; Oxybelidae: Notoglossa americana $\mathrm{Rb}$., type, cressonii $\mathrm{Rb}$., type, emarginata Say, frontalis Rb., type, inomata Rb., type, Oxybelus laetus Say, 
niger Rb., type, packardii Rb., type, 4-notatus Say: Pomphredonilut: Cemonus inornatus Saty, Mimesal eresonnii Pe., P'sen tilialis Cr.; Philanthilae (ineludine Cereridae): Cereeris astarte Banks. bicomuta Guerin, elymata Dh., compateta Cr., compar C'r., echo Mickel, finitima Cr., fulvipes ( r. (fulvipeslienlata Rehlet.), fumipennis Say, insolita C'r.. kemuienttii Cr., prominns Panlis, raui Rohwer (irontata Say?), robertsonii Fox, colype, rufinoda Cr., Eucereris zonatus Say, Philanthus politus Say, punelatus Say, ventilabris F.: Pompilidae: Igeniella aceepta C'r., lomerula Cr. petiolata Cr., Anoplius illinoensis Rb., lypt, virginiensis Cr., Arachnoproctoms ferrugineus Say, Batizonus aleridus Sn., Coropales bipunetata say. eleugans Cr., fraterna Sm., fulvipes Cr., robinsonii Cr., Cryptocheilus agenioides Fox, enteus Say, fulgifrons ('r., Episyron biguttatus F., Lophopompilus atrox Dh., philadelphieus Lpr., Planieeps feralis Cr., nieer Cr., Poevilopompilus interruptus Say, narus Cr., Pompiloides amerieanus Bv., divisus Cr. marginatus Say, subviolaceus Cr., Proenenis arenatus Banks, Prioenemoides fulvieornis Cr., terminatus Say, unifasciatus Say, Psammochares pretiosa Banks, relativus Fox, colype, seelestus Cr., tunebrosus Cr., Pseudagenia mullipes Say, Soploropompilus ingenuus Cr.; Saplygidae: Sapyga centrata Say; Scclionidae: Prosaeantha illinoensis Ash. MS, Teleas lineaticeps Ash.; Scolizlae: Campsomeris eonfluenta Say, Flis interrupta Say, obseura F. thoraciea Fox, type, 5-cincta F., Scolia bicineta F., nobilitata F.; Sphecidae: Ammobia ichneumonea L., nuda Fernald, pennsylvaniea I., Chalybion eaeruleum L., Chlorion (aeruleum I)r., Isodontia apicalis Sm.. macrocephala Fox, Palmodes dimidiata Dr. Priononyx atrata Lp., thomae F., Seelipluron eementarius Dr., Sphex gracilis Lp., nierreans Dh., pictipennis Walsh, procera Klue, vulgaris Cr.; Tenthrolinidae (including Xrelidae): Dolerus aprilis Nr., arvensis say (unieolor Bv.), bienlor Bv., sericeus Say. Hylotoma humeralis Bv., macleayi Leach, Macroplya epinota Say, pleuricineta Nr. (nebraskensis Rolıwer), Maeroxyela ferruginea Say. Monophadnus medius Nr., Nematus lufeofergum Nr., vertebratus Say, Opisthoneura albidovariata Nr., Pseudosiobla exeavata Nr.: Thynnilat: Methoea stygia Say: Tiphiular: Tiphia elypeata Rb., type, illinoensis Rb., type, inoruata Say, punetata Rh., type, robertsoni Malloch, type, transversa Say, vulgaris Rh.. type: Trypoxylidae: Trypoxylon elavatum Say, frigidum Sim., triduntatum Pe.; Vespidat: Polistes ammularis L., pallipes Lp., rubiginosus L.p., variatus Cr., Tespula euneata F., germaniea F., maculata $L_{\text {. }}$, vidua Ss. 


\section{Diptera. (446)}

Agromyzidae: Agromyza aeneoventris Fl., latipes Mg., Aulacigaster rufitarsis Mc., Eusiphona mira Cq., Leucopis nigricornis Egger (americana Malloch), Lobioptera indecora Lw., Milichiella lueidula Beck, Phytomyza palpalis Cq. MS.; Anthomyidae: Anthomyia pluvialis L., radicum L., Anthomyiella pratincola Panzer, Calythea albicincta Fl., Coenosia antennalis Stein, antica Wk. (leucoprocta Wd.), fuscopunctata Mc. (ovata Stein), lacteipennis Mg., sexnotata Mg., Hammomyia paludis Johansen, Homalomyia canicularis L., prostrata Rossi (incisurata Zett.), Hydrophoria ambigua Fl., Hydrotaea houghi Malloch, Hyetodosia rufitibia Stein, Hylemyia dilatata Malloch, inornata Stein, juvenalis Stein, laevis Stein, plumosa Cq., type, Limnophora narona Wk., Lispa albitarsis Stein, consanguinea Lw., uliginosa Fl., Mydaea diruta Stein, fusea Stein, socialis Stein, Ophyra leucostoma Wd., Phorbia acra Wk., cinerella F., platura Mg., Proboscimyia siphonina Bigot; Bibionidae: Bibio albipennis Say, femoratus Wd., pallipes Say, Dilophus obesulus Lw., serotinus Lw., Scatopse notata L., pulicaria Lw., Bombyliidae: Aldrichia ehrmanii Cq., Anthrax alternata Say, fulvohirta Wd., halcyon Say, hypomelas Mc., palliata Lw., parvicornis Lw., sinuosa Wd., Argyramoeba albofasciata Mc., oedipus F., Bombylius atriceps Lw., azaleae Shannon, major L., pulchellus Lw., Exoprosopa decora Lw., emarginata Mc., fasciata Mc., fascipennis Say, Geron calvus Lw., rufipes Mc., Lepidophora lepidocera Wd., Oncodocera leucoprocta Wd., Phthiria aldrichi Johnson, cincta Cq. MS, sulphurea Lw., Sparnopolius fulvus Wd., Systoechus vulgaris Lw., Systropus macer Lw., Thlipsogaster ater Cq., Toxophora amphitea Wk., Borboridae: Borborus equinus Fl.; Chironomidae: Ceratopogon fusculus Cq., websteri Cq., Conopidae: Conops brachyrrhynchus Mc., sylvosus Wl., xanthopareus Wl., Dalmannia nigriceps Lw., Myopa pilosa Wl., vesiculosa Say, Oncomyia loraria Lw., Physocephala texana Wl., tibialis Say, Robertsonomyia palpalis Rb., type, Stylogaster biannulata Say, neglecta Wl., Zodion fulvifrons Say, nanellum Lw., obliquefasciatum Mc.; Culicidae: Aedes vexans Mg., Culex impiger Wk. (nigripes Zett.), stimulans Wk. (cantans Mg.); Dexiidae: Myocera cremides Wk., Ptilodexia abdominalis Ds., Scotiptera parvicornis Tn. MS; Drosophilidae: Drosophila varia Wk.; Empididae: Brachystoma robertsonii Cq., type, Empis avida Cq., type, compta Cq., distans Lw., humilis Cq., type, levicula Cq., type, loripedis Cq., type, nuda Lw., otiosa Cq., type, Pachymeria pudica Lw., Parempis (MS) clausa Cq., Rhamphomyia angustipennis Lw., exigua Lw., fumosa 
Lw., gilvipilosa $\mathrm{C}_{4} .$, type, irregularis Lw., limbata Lw., mutabilis Lw., piligeronis Cq., type, priapulus Lw., rava Lw., ravida Cq., type, sordida Lw., tersia Cq., Tachydromia pusilla lww, Ephydridue; Notiphila unicolor Lw., Oehthera lauta Wheeler, pred.; IItteroneuridue: Clusia lateralis Wk.; Le ptidae: Hilarimorpha mikii W1., type; Lonchatidue: Lonchaea parvieornis Mg., aberrans Malloch, cotype: Midnidue: Mislas elavatus Dr., tibialis Wd.; Muscidur: Compsomy ia macellaria F., Graphomyia americana Ds. (matulata Seopoli), Luvilia caesar L., sericata Mg., sylvarum Mg., Morellia mieans Me., Musea domestiea L., Museina assimilis Fl., Myospilat meditabunda F., Phormia regina Mrg., terraenovae Ds., Pollenia rudis F., Protocalliphora splendida Me., Pseudopyrellia cornicina F., Stomoxys ealeitrans L.; Mycetophilidue: Dynatosoma thoraciea Cq., type, Eugnoriste occidentalis Cq., Sciara atrata Say, inconstans Fiteh, vulgaris Fiteh; Nemestrinidue: Rhynchocephalus sackeni W1.; Ortulidue: Camptoneura pieta MIe., Chactopsis aenea Wd., Euxesta notata Wd., Rivellia pallida Lw., 4 -faseiata Mc., viridulans Ds., Seoptera eolon Lw.; Oscinidae: Chlorops approximatonervis Zett., assimilis Me., mellea Lw., proxima Say, pulverea Cq., versicolor Lw., Elachiptera estata Lw., flavida Wl., Hippelates flavipes Lw., plebejus Lw., pusio Lw., Meromyza amerieana Fiteh, Oscinis coxendix Fitch, soror Me., trigramma Lw., Siphonella cinerea Lw., inquilina Cq., oscinina Fl.; Phoridae: Phora fungicola Cq., Trineura aterrima F.; Pipunculidae: Chalarus spurius Fl.; Psilidae: Chyliza apicalis Lw.; Sapromyzidae: Sapromyza longipennis F., vulgaris Fiteh; Sarcophagidue (Calliphora and Cynomyia belong to Muscidae): Boetteheria eimbicis Tn., Calliphora erythrocephala Mg., vomitoria L., Cynomyia eadaverina Ds., Ifelieobia helieis Tn., 4-setosa Cq., Metoposareophaga pachyprocta Parker, Sareophaga alcedo Al., aldrichi Parker, assidua Wk., bullata Parker, eimbieis (pallinervis Thompson), dux sarracenioides Al., haemorrhoidalis Fl., hunteri Hough, impar Al., marginata Al., sinuata Mg., utilis Al.; Scutophagidae: Cordylura munda Lw., Seatophaga fureata Say; Scenopinidae: Scenopinus nubillipes Say; Sciomyzidue: Sciomyza humilis Lw., Tetanocera pietipes Lw. (umbrarum L.); Sepsidae: Nemopoda minuta Wd., Piophila easei L., Prochyliza xanthostoma Wk., Sepsis violacea Mg., Themira putris Lw.; Simuliidue: Simulium peeuarum Rl.; Strutiomyidue: Allognosta fuseitarsis Say, ohseuriventris Lw., Mierochrysa polita I., Nemotelus glaber Lw., Odontomyia eineta Ol., nigrirostris Lw., pubeseens Day, Sargus viridis Sity, Stratiomyia apieula Lw.. disealis Lw., meigenii Wd., quaternaria Lw., unilimbata Lw.; 
Syrphidae: Allograpta obliqua Say, Baccha aurinota Wk. (fascipennis 'Wd.), clavata F., fuscipennis Say, tarchetius Wk., Brachyopa vacua O. S., Brachypalpus frontosus Lw. (oarus Wk.), rileyi Wl. (metallifera Bigot), Ceria abbreviata Lw., Chalcomyia aerea Lw., Chilosia capillata Lw. (caltha Shannon), eyanescens Lw., pallipes Lw., petulea Wl., puctulata Hunter, Chrysochlamys dives O. S., Chrysogaster nigripes Lw., ontario Curran, Criorhina decora Mc., intersistens Wk., umbratilis Wl., Didea faseiata fuscipes Lw., Eristalis aeneus Scopoli, bastardi Mc., brousi Wl. (meigenii Wd.), dimidiatus Wd., flavipes Wk., latifrons Lw., tenax L., transversus Wd., vinetorum F., Helophilus divisus Lw., laetus Lw., latifrons Lw., similis Mc., Mallota cimbiciformis Fl., illinoensis Rb., type, posticata F. Melanostoma mellinum L., obscurum Say, Merapioidus villosus Bigot, Mesogramma geminata Say, marginata Say, polita Say, Milesia ornata F. (virginiensis Dr.), Myiolepta nigra Lw., strigilata Lw., varipes Lw., Orthoneura nitida Wd., pictipennis Lw., Paragus bicolor F., tibialis Fl., Pipiza femoralis Lw., festiva Mg. (albipilosa Wl.), nigribarba Lw. (apisaon Wk.), pistica :Wl., pubescens Lw., pulchella Wl. (Pipizella banksi Curran), Plátychirus hyperboreus Staeger, quadratus Say, Psilota buccata Míc., Pterallastes thoracicus Lw., Rhingia nasica Say, Sericomyia chrysotoxoides Mc., Sphaerophoria cylindrica Say, Sphegina campanulata Rb., type, rufiventris Lw., Spilomyia hamifera Lw., longicornis Lw., 4-fasciata Say, Syritta pipiens L., Syrphus americanus $\mathrm{Wd}$., arcuatus Fl., ribesii F., torvus O. S., Temnostoma trifasciata Rb., type, Teuchocnemis lituratus Lw., Tropidia albistylum Mc., mamillata Lw., quadrata Say, Volucella evecta Wk., fasciata Mc., vesiculosa F., Xanthogramma emarginata Say, felix O. S., flavipes Lw., Xylota angustiventris Lw., bicolor Lw., chalybea Wd., fraudulosa Lw. (baton Wk.); Tabanidae: Chrysops indus O. S., striatus O. S., Tabanus lineola F., Therioplectes illotus O. S.; Tachinidae: Actia pilipennis Fl., Alophora aeneoventris Wl., fumosa Cq., grandis Cq., cotype, pulverea Cq., Amobia distincta Tn., type, Apinops atra Cq., type, Araba tergata Cq., Archytas analis F., aterrima Ds., hystrix F., Belvosia bifasciata F., unifasciata Ds., Biomyia georgiae B. B., Blepharipeza leucophrys Wd., Brachycoma intermedia Tn., type, sarcophagina Tn., type, Celatoria diabroticae Shimer, Ceratomyiella conica Tn., type, Chaetogaedia analis Wulp (aeroglossoides Tn., type), crebra Wulp, Chaetophleps setosa Cq., Chaetoplagia atripennis Cq., Cistogaster occidua Wk., pallasii Tn. Clytiomyia flava Tn., type, Cryptomeigenia theutis Wk., Cyphocera fucata Wulp, Distichona auriceps Cq., varia W.ulp, Ennyomma clistoides Tn., type, globosa Tn., 
nigrifrons T'n., type, Epalpus signiferus Wk., Epigrimyia floridensis T'n., genieulata 'T'n., type, illinoensis lib., type, polita 'Tn., Enacemyia tibialis Cq., Eumyotheria illinoensis Tn., type, Euphorocera einerea Wulp, elaripennis Mc. (edwardsii WL.), Euryceromyia robertsonii Tn., lype, Euthera tentatrix Lw., Exorista confinis Fl., nigripalpis Tn., pyste Wk., Frontinat aletiae Ri., archippivora Wk., frenchii Wl., Gonia eapitata Dg., exul Wl., (iymuochaeta aleedo Lw., Gymnoprosopa clarifrons Tn., type, Gymnosoma fuliginosa Ds., Hilarella aristalis Cq., type, Hyalomyodes triangulifera Lw., Hyalurgus johnsoni Tn., Hypostena barbata $\mathrm{C}_{q}$., dumningii Cq., indecisa 'Tn., lype, variabilis Cq., Leskia analis Cq. (similis Tn.), Leskiomima prima Rb. MS, seeunda Rb., MS, Leueostoma atra T'n., type, neomexicana T'n., nigricornis 'Tn., siphonina Rh. MS, Linnaemyia compta Fl., Macquartia pristis Wk. (halisodotae Tn.), Masiphya brasiliana B. B., Metopia leueocephala Rossi, Neofischeria flava T'n., Ocyptera argentea 'Tn., nigra Al., dosiades Wk., lata Rb. MS (vulgaris $\mathrm{Al}$.), tenuis lib. MS (enchenor Wk.) Opsidia gonioides Cq., Pachyophthalmus floridensis Tn., signatus Mg. (mixed with distortus, aurifrons Tn., type), Panzeria radicum F. (aldrichi Tn.), Paradidyma singularis Tn., type, Peleteria robusta Wd., Phasioclista metallica T'n., type, Phorantha asteris Rb. MS, humeralis Rb., type, magna Rb. MIS, purpuraseens Tn., type, robertsonii Tn., type, Phorichaeta sequax Wl., Phyto elesides Wk. (anomala Tn.), Polidea areos Wk. (americana Tn., type), Senotainia rubriventris Mce, trilineata Wulp, Siphona geniculata De., Siphoplagia anomala Tn., flaceidirostris Rb. MS Spallanzania hesperidarum Wl., Sturmia distincta Wd., fraudulenta Wulp, inquinata $W_{1} u$, nigrita Tn., type, plyeciodis Cy., Tachina mella Wk., robusta Tn., rustica Fl., Trichopoda lanipes F., pennipes F., plumipes $\mathrm{F}$. (trifasciata Lw.), Triehophora rufieauda Wulp, Viviania lachnosternae Tn., Winthemia 4-pustulata F.. Xanthomelana arcuata Say; Tipulidue: Geranomyia eanadensis Westwood; Trypetidue: Euaresta acqualis Isw., bella Lw., Plagiofona obliqua Say, Trypeta eulta Wd., finalis Lw., humilis Lw., solaris Lw.

\section{Lepidoptera. (99)}

Agaristidne: Alypia oetomateulata F.; Aretioitat: Callimorphat fulvieusta Clemens (eolona Hb.), Utetheisa bella L.; Clenuchidat: Scepsis fulvicollis IIb. : Crambidae: Crambus lagneatellus Clemens.; Hespreriidue: Aehalarus lyeidas S. \& A., Amblyseirtes samuset (hemon) Se., Aneyloxypha numitor F., Atalopedes huron Ed. (campestris Ps.1, Atrytone delaware (logan) EA., zabulon B. L., 
Epargyreus tityrus F., Euphyes metacomet Harris (vestris Bs.), verna Ed., Hesperia montivaga Reakirt (syrichtus F.), Hylephila phylaeus Dr., Limochores manataaqua Sc., taumas F. (cernes B. L.), Pamphila dion Ed., hobomok Hr., Pholisora catullus F., hayhurstii Ed., Polites peckius Kirby, Thanaos brizo B. L., icelus Lintner, juvenalis F., martialis Sc., persius Se., Thorybes bathyllus (daunus Cm.), pylades Sc.; Lycaenidae: Chrysophanus thoe B. L., Cyaniris pseudargiolus B. L., Eupsyche m-album B. L., Everes comyntas Godart, Heodes hypophlaeas Bs., Strymon titus F., Thecla calanus Hb., sylvinus Bs. (acadica Ed.), Uranotes melinus Hb.; Noctuidae: Acontia candefacta Hb., Agrotis ypsilon Rott. Carneades velleripennis Grote, Drasteria erechtea Cm., erichto Gn. (crassiuscula Haw.), Feltia subgothica Stephens (ducens Wk.), Heliothis armiger Hb. (obsoleta F.), dipsaceus L. (phloxiphaga G. \& R.), Leucania albilinea Hb., Plusia precationis Gn., simplex Gn. (falcifera Kirby), Schinia jaguarina Gn., Spragueia leo Gn.; Nymphalidae: (including Danaidae, Satyridae, Libytheidae): Anosia plexippus L. (Danaus archippus F.), Argynnis cybele F. (mixed with aphrodite F.), Basilarchia archippus Cm., astyanax F., Cercyonis alope F., Charidryas nycteis D. \& H., Euphydryas phaeton Dr., Euptoieta claudia Cm., Euvanessa antiopa L., Hypatus bachmanni Kirt., Junonia coenia Hb., Neonympha eurytus F., Phyciodes tharos Dr., Polygonia comma Harris, interrogationis F., Speyeria idalia Dr., Vanessa atalanta L., cardui L., huntera F. (virginiensis Dr.) ; Papilionidae: (including Pieridae) : Callidryas eubule L., Euphoeades troilus L., Eurema lisa B. L. (euterpe Men.), Eurymus philodice Godart (mixed with eurytheme), Heraclides cresphontes $\mathrm{Cm}$., Iphiclides ajax L. (marcellus Cm.) Jasoniades glaucus L., Laertias philenor L., Nathalis iole Bs., Papilio asterias F. (polyxenes F.), Pieris repae L., Pontia protodice B. L., Xanthidia nicippe Cm., Zerene caesonia Stoll; Pyraustidae: Loxostege similalis Gn., Nomophila noctuella Voll.; Pyromorphidae: Harrisina americana Guerin; Sesiidae: Carmenta pyralidiformis Wk., Sesia pictipes G. \& R., 6-fasciata Ed. (bassiformis Wk.); Sphingidae: Chaerocampa tersa L., Deilephila lineata F., Hemaris axillaris G. \& R. (diffinis Bs.), thysbe F., Philampelus pandorus Hb. (satellitia Dr.), Protoparce celeus Hb. (5-maculata Haw.), Sphinx eremitus Hb.

\section{Coleoptera. (153)}

Anthicidae: Corphyra labiata Say, terminalis Say; Bruchidae: Bruchus bivulneratus Horn, eruentatus Horn, discoideus Say, exiguus Horn (horni Pic.), hibisci Ol., mimus Say, musculus Say, obsoletus Say (obtectus Say); Buprestidae: Acmaeodera culta 
Weber (tubulus F.), pulchella IIr., Agrilus difficilis Gory, egenus Gory; Calandryidae: Rhodobaenus 13-punetatus 111.; Carubidae: Callida punetata Le., Lebia viridis Say; Cerambycidue: Aemaerops nigripennis Le, direeta Nw., Batyle suturalis Say, Callimoxys fuseipemnis Le., sanguinieollis Ol., Cyllene decorus Ol., robiniae Fr., Euderees pieipes F., Leptura exigua Nw., pubera Say, vittata Gr., Molorehus bimaculatus Say, Strangalia famelica Nw., 'T'etrapes tetraphthalmus Fr., Typocerus badius Nw., lugubris Say, sinuatus Nw., relutinus Ol.; Chrysomelidue: Anomoea laticlavia Fr., Babia 4-guttata Ol., Cerotoma caminea F. (trifurcata Fr.), Chrysomela similis Rogers, Cryptocephalus insertus Hald., Diabrotica atripennis Say, 12-punetata F., longieornis Say, vittata F., Disonyeha limbicollis (pennsylvaniea) pallipes Crotch, Galeruca tubereulata Say, Glyptina spuria Le., Haltica carinata Gr., Lina lapponiea I., Luperaltiea fuseula Le., Mantura floridana Croteh, Orsodachna atra Ahrens, Pachybrachys atomarius .II., Rhabdopterus picipes Ol., Trirhabda tomentosa L.; Cleridae: Enoplium t-punctatum Say; Coccinellidue: Coecinella 9-notata Hr., sanguinea L., Hippodamea glacialis F., parenthesis Say, 15-maculata Mul. (eonvergens Guerin), 13-punctata L., Megilla maculata Dg. (fuscilabris Mul.), Seymnus consobrinus Le., terminatus Say; Curculionidae: Apion nigrum Hr., Centrinites strigicollis Casey, Centrinus perseillus Gyll., picummus IIr., seutellum-album Say, Idiostethus subealvus Le., tubulatus Say, Limnobaris prolixa Le., Listronotus eaudatus Say; Dermestidae: Anthrenus musacorum L. (eastaneae MIl.), serophulariae L., Attagenus piceus Ol., Cryptorhopalum haemorrhoidale Le., triste Le., Orphilus glabratus F. (ater Er.); Elateridne: Agriotes insanus Candeze, Limonius griseus Bv. (propexus ('andeze), Melanotus communis Gyll., Sericosomus silaceus Say; Erotylidat : Languria mozardi Latr.; Itisteridae: Hister amerieanus Paykull, subrotundus Say ; Lampyridae: Calopteron retieulatum F., Chauliognathus marginatus F., pennsylvanieus Der., Ditemuus bidentatus Say, Ellychnia corrusea L., Photinus pyralis I., Podabrus brumnicollis Le., rugulosus Le., tomentosus Say, Pyractomena angulata Say, Telephorus bilineatus Say, dentiger Le, flavipes Le., scitulus Say : Lutrididue: Corticaria distinguenda Comolli; Melachidae: Anthocomus erichsoni Le. Attalus scincetus Say, Collops 4-maculatus F.. Melyris eribrata Le.; M.landryidue: Canifa pallipes Ml.. Nothus varians Le.; Melvidue: Epieauta cinerea Fr, pennsylvaniea Dg., trichrus Pallas, vittata Fr., Macrobasis unicolor Kirby, demognatha vittigera Le., Pyrota germari Haldeman, mylabrina Cherrolat, terminata Le.; Mordellidue: Mordella marginata MIl, meláena (ir., s-punetata $F$., sentellaris $F$., triloba Say, Mordellistena 
aspersa Ml., biplagiata Hel., comata Lc., grammica Lc., limbalis Ml., lutea Ml., marginalis Say, ornata Ml., pubescens F., tosta Le., Pentaria trifasciata Ml.; Nitidulidae: Carpophilus brachypterus Say, Cercus abdominalis Erichson, Epurea labilis Erichson, truncatella Mannerheim; Oedemeridae: Asclera puncticollis Say, ruficollis Say, Oxacis thoracica F.; Rhipiphoridae: Myodites fasciatus Say, Rhipiphorus dimidiatus F., flavipennis Lc., limbatus F.; Rhynchitidae: Eugnamptus angustatus Hr.; Scarabaeidae: Euphoria fulgida F., sepulchralis F., Hoplia trifasciata Say, Macrodactylus angustatus Bv., Onthophagus pennsylvanicus Harold, Trichius affinis Gory, piger F., Valgus canaliculatus F.

HEMIPTERA.

Acanthiidae: Triphleps insidiosus Say; Berytidae: Corizus lateralis Say, Harmostes reflexulus Say; Capsidae: Calocoris rapidus Say, Lopidea media Say, Lygus pratensis L., Phytocoris scrupeus Say, Plagiognathus obscurus Uhler; Coreidae: Alydus eurinus Say, pilosulus H.S., Chariesterus antennator F.; Corimelaenidae: Corimelaena lateralis F., pulicaria Gr.; Cydnidae: Canthophorus einctus Bv.; Lygaeidae: Lygaeus turcicus F., Melanocoryphus bicrucis Say, Oncopeltus fasciatus Dallas, Ortholomus longiceps Stal; Nabidae: Coriscus ferus L.; Pentatomidae: Euschistus fissilis Uhler (euschistoides Vol.), ictericus L., variolarius Bv., Podisus spinosus Dallas; Phymatidae: Phymata wolffii Stal, pred.; Reduviidae: Sinea diadema F.

\section{NEUROPTERA}

Chrysopidae: Chrysopa plorabunda Fitch.

\section{VISITORS}

\section{Acanthaceae}

Dianthera americana $(5: 65-6)$.-Blooms June 8-August 23; 58 visitors observed, June 11-July 5 ; Mas, $R$.

Long-tongued Bees (24)-Ap.: Apis ab; Bomb.: Bombias separatus $\not, \mathrm{fq}$, Bombus americanorum $q$, impatiens $\not$; Ceratin.: Ceratina $\delta^{\top} q$, ab; Megachil.: Osmiini (q) : Alcidamea simplex sc, fq, Hoplitis sc, Osmia distincta; Megachilini : Cyphopyga ${ }^{\lambda}$, Megachile addenda $q$, se, brevis $\widehat{\sigma}$, generosa $\widehat{\sigma}$, Sayapis pugnata $\widehat{\sigma}$; Coelioxyini : Coelioxys 8-dentata $\delta^{\top} q$, sayi $\delta^{\gamma}$ ㅇ, fq; Nomad.: Centrias americanus $q$; Epeol.: Epeolus bifasciatus $\sigma^{\lambda}$, Triepeolus concolor $\delta$; Eucer.: Florilegus $\delta$, ab, Melissodes bimaculata $\delta \uparrow$, ab, variabilis $\widehat{c}$, Tetralonia dilecta $q$, rosae $\delta$; Anthophor.: Anthemoessa or $q$, fq. 
Short-tongued Bees (11) - Halict.: Agapustemon radiatus of $q$, viridulus $q$. Chloralietus pilosus $q$, e, sparsus $q$, se, versatus $q$, se, ab, Halictus lerouxii of ${ }^{+}, a b$, Odontalictus of ㅇ. Oxystoglossa enfusa

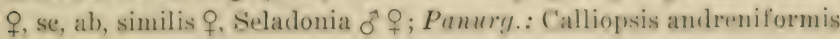
$\delta$ o, ab.

Other Hymenoptera (3)-Siphec: Sphex nigricans; Bembic: Bembix spinolae; Scoli.: Campsomeris.

Diptera (12) - Syrph.: Allograpta f, n, Eristalis teuax sf, Helophilus laetus $1, n$, Mesogramma marginata $f, n$, sphaerophoria $f, n$. Syritta, Tropidia quadrata; Bombyli.: Anthrax parvicornis. Pombylius azaleae, atriceps fq; Conop.: Physoeephala tibialis, Zodion fulvifrons.

Lepidoptera (8)-Nymphal.: Charidryas; Lycaen.: Cyaniris; Papilion.: Pieris; Ilesperi.: Epargyreus, Euphyes metacomet, Pholisora eatullus, Thanaos martialis, Thorybes bathyllus.

Ruellia ciliosa.-Blooms May 26-September 20; 10 visitors observed, May 29-August 2; Ma, R.

Long-tongued Bees (6)-Bomb.: Bombus americanorum $q$, impatiens $q$; Megachil.: Merachile brevis $q$, c, also eutting petals,

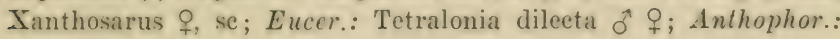
Amegilla $\sigma^{\top}$.

Short-tongued Bees (1) -II alict.: Agapostemon viridulus $q, \mathrm{c}, \mathrm{n}$.

Other IIymenoptera (1)-Eumen.: Leionotus dorsalis, perforating.

Diptera (2, f, n)-Syrph.: Allograpta; Bombyli.: Anthrax sinuosa.

Ruellia strepens.-Blooms May 22-September 13; 1 visitor observed, June 16 ; Ma, R.

Long-tongued Bees (1)-Eucer.: Tetralonia dileeta ㅇ.

\section{ACERACEae (anemophilous)}

Acer negundo.--One visitor observed, April 18, 24.

Long-tongued Bees (1)-Ap.: Apis c, ab.

Acer saccharinum.-One visitor observed, April 10.

Long-tongued Bees (1)-Ap.: A pis c, ab.

Acer saccharum.-April 4 and 13 the following 22 species were taken at exuding sap, 5 never taken on flowers.

Long-tongued Bees (1)-Ap.: Apis ab.

Short-tongued Bees (1)-Andren.: Trachandrena rugnsa of. 8.

Other IIymenoptera (2)-Bracon.: Earinus 7; Tenthredin.: Dolerus sericeus 2. 
Diptera (15) -Syrph.: Helophilus similis 1, Syrphus ribesii 3; Tachin.: Gonia eapitata fq; Sarcophag.: Calliphora erythrocephala 1, vomitoria 3, Cynomyia 1, Helicobia 4-setosa 1; Musc.: Lucilia caesar 4, Muscina 2, Pseudopyrellia 4; Anthomy.: Hydrotaea 3, Hyetodosia 25; Agromyz.: Aulacigaster 2; Scatophag.: Scatophaga 12 ; Seps.: Prochyliza 10.

Lepidoptera (3)-Nymphal.: Euvanessa 4, Polygonia comma 2, Vanessa atalanta 2.

\section{AtzoACEAE}

Mollugo verticillata (int, 5: 274).-Blooms June 25-October 12 ; 13 species and 29 individuals observed, July 16, August 11, 21; $\mathrm{Mi}, \mathrm{W}$.

Short-tongued Bees $(5: 13)$-Halict.: Chloralictus pilosus $\delta, 1$, sparsus $q$, se, 4 , tegularis $o^{\top} q, 3$, versatus $o^{\Uparrow} q$, se, 4 , Seladonia $\sigma^{\lambda}, 1$.

Other Hymenoptera (1:3)-Philanth.: Cerceris finitima 3.

Diptera (6:9)—Syrph.: Mesogramma marginata sf, 4, Paragus tibialis 1, Pipiza pulchella 1 ; Conop.: Zodion nanellum 1 ; Sarcophag.: Helicobia 4-setosa 1; Musc.: Pseudopyrellia 1.

Coleoptera (1:2)-Malach.: Collops 2.

\section{Alismaceae}

Alisma plantago aquatica.-Blooms July 4-August 13; 13 species and 101 individuals observed, July 20, 21, 1909; Mi, W.

Short-tongued Bees (6:52)-Halict.: Agapostemon radiatus $\hat{\sigma}$, 1 , Chloralietus coreopsis + , 1 , cressonii $\sigma^{1}, 3$, sparsus + , 8 , tegularis , , 31, Oxystoglossa similis,+ 8.

Diptera (7:49)-Syrph.: Mesogramma marginata 32, Paragus bicolor 2, tibialis 2, Sphaerophoria 10, Syritta 1; Tachin.: Gymnoprosopa 1 , Senotainia rubriventris 1 .

Sagittaria latifolia.-Blooms July 1-September 25; 122 individuals, belonging to 52 species, observed July 25-August 30; Mi, W.

On staminate flowers $(24: 42)$ :-

Long-tongued Bees (2:2, )-Ceratin.: Ceratina o, 1; Megachil.: Megachile brevis + , se, 1.

Short-tongued Bees $(5: 18, q$, sc) - Halict.: Chloralictus sparsus 5, versatus 7, Evylaeus nelumbonis 1, Odontalictus 4, Oxystoglossa confusa 1.

Other Hymenoptera (4:4)-Eumen.: Leionotus anormis 1, ziziae 1; Larr.: Tachytes aurulentus 1; Nysson.: Pseudoplisus phaleratus 1 . 
Diptera $(8: 12)-S y r p h:$ Mesogramma marginata 1, Orthoneura nitida f. 2. Rhingia 1 ; Empid.: Parempis 1 ; Bombyli.: Sparnopolius 2; Turhin.: Epigrimyia genieulata 1; Muse.: Lucilia serieata 1 ; Anthomy.: Limnophora 3.

Lepidoptera (3:3)-P'upilion.: Charidryas 1; Lycaen.: Chrysophanus 1; Hesperi.: Limochores taumas 1.

Coleoptera (2:3)-Chrysomel.: Diabrotica 12-punetata 1; Coccinell.: Megilla $f$, in cop, 2.

On staminate and pistillate flowers $(17: 66)$ :-

Long-tongued Bees $(2: 13)-A p .: A_{\text {pis }} 3$; Bomb.: Bombus amerieanorum $\not{q}$, se, 10.

Short-tongued Bees $(t: 18)$-Hulict.: Agapostemon viridulus of, 5. Chloralietus pilosus $q$, se, 3, Evylaeus pectoralis of 9 , se, 8, Oxystoglossa similis $q$, se, 2.

Other Hymenoptera $(3: 12)$-Scoli.: Elis interrupta 2, 5-eineta 7; Eumen.: Leionotus arvensis 3.

Diptera $(4: 10) \rightarrow$ Syrph.: Syrịta 2; Tuchin.: Archytas analis 3, Cyphocera 2; Musc.: Lucilia sylvarum 3.

Lepidoptera (3:9)-Tymphal.: Phyciodes 3; Hesperi.: Ancyloxypha 2 ; Ctenuch.: Scepsis 4.

Coleoptera $(1: 4)$-Lampyr.: Chauliognathus pennsylvanieus 4.

On pistillate flowers $(11: 14)$ :-

Short-tongued Bees (2:2)-Inulict.: Augochlora fervida o, 1, viridula + , 1.

Other Iywanoptera (3:4)-Sphec.: Priononyx atrata 1; Philanth.: Cerceris compacta 1 ; Vesp.: Polistes pallipes 2.

Diptera (5:7)-Tuchin.: Spallanzania 1, Trichophora :3; Sarcophag.: Helieobia helieis 1 , Sareophaga sinuata 1 ; Musc.: Lueilia caesar 1.

Lepidoptera (1:1)-Papilion.: Eurymus 1.

\section{Amarantiraceae (anemophilous)}

Amaranthus spinosus (int).-One visitor observed, July 19.

Long-tongued Bees (1) - A p.: Apis se, fq.

\section{A maryluidaceae}

Hypoxis hirsuta $(5: 69)$.-Blooms April 23-June 12 ; 16 visitors observed, May $10-19 ; \mathrm{Mi}, \mathrm{Y}$.

Long-tongued Biees (4,, , c)-Ceratin.: Ceratina ab; Megachil.: Osmia atriventris, illinoensis $\mathrm{f} q$, distincta.

Short-tongued Bees (9, \&. e)-Malict.: Chloralietus eressmnii, sparsus, tegularis, versatus, Curtisapis eoriaeea, Dialictus anomalus type. Evylacus peotoralis, Odontalietus, Oxystoglonsa similis ab. 
Diptera (2, f)-Syrph.: Mesogramma marginata, Sphaerophoria. Coleoptera $(1, \mathrm{f})-$ Buprest.: Acmaeodera culta.

\section{ANACARdiaceae}

Rhus canadensis $(9: 161-3)$.-Blooms April 4-May 2; 33 visitors observed, April 4-19; Mis, Y.

Long-tongued Bees (2)-Ceratin.: Zaodontomerus ô; Nomad.: Gnathias cuneatus o $q$, fq.

Short-tongued Bees (21)-Halict. () : Agapostemon texanus, Chloralictus cressonii, foveolatus, sparsus, zephyrus fq, Curtisapis forbesii fq, Evylaeus foxii fq, Odontalictus, Oxystoglossa confusa; Collet.: Colletes inaequalis $\sigma^{\wedge}$; Andren.: Andrena carlini $\delta^{1}+\mathrm{f}, \mathrm{fq}$, erythrogastra $q$, mandibularis $\delta \mathcal{f}, f q$, salictaria $q$, tridens $\delta$, Opandrena cressonii $\delta$, miserabilis $\delta q$, fq, Trachandrena claytoniae $\delta^{\lambda}+$, ab, forbesii $\delta^{\lambda}+, \mathrm{fq}$, mariae $\sigma^{\lambda}, \mathrm{fq}$, rugosa $\delta^{\lambda}+$, ab.

Other Hymenoptera (1)-Ichneumon: Lampronota coxalis.

Diptera (9)—Syrph.: Eristalis dimidiatus, Syrphus americanus fq, ribesii; Empid.: Rhamphomyia priapulus; Tachin.: Gonia capitata fq; Sarcophag.: Cynomyia; Musc.: Pseudopyrellia fq; Lonchae.: Lonchaea aberrans; Sciomyz.: Tetanocera.

Rhus copallina.-Blooms July 19-August 10; 74 visitors observed, July 26-28; Pol (Mis), Y.

On pistillate flowers:-

Long-tongued Bees (8)-Ap.: Apis ab; Bomb.: Bombias separatus ఫ̛; Megachil.: Trypetini: Neotrypetes truncatus ô; Megachilini : Megachile generosa $\delta^{\lambda}$, mendica $\delta^{\lambda}$; Coelioxyini : Coelioxys 8-dentata $\delta^{7}$ 우, sayi $\delta^{\top}$; Eucer.: Melissodes bimaculata $\delta^{7}$.

Short-tongued Bees (15)-Halict.: Agapostemon radiatus o 0 , Augochlora fervida $\delta^{\lambda}$, Chloralictus pilosus $\delta$, sparsus $q$, ab, versatus $\sigma^{\lambda} q$, ab, zephyrus $q$, fq, Halictus lerouxii $\delta^{\lambda} q$, ab, parallelus $o^{\lambda}+$, Oxystoglossa confusa $q$, similis $\delta^{\lambda}$, Seladonia $\delta^{\lambda}+$, ab; Sphecodini : Drepanium $q$, Sphecodes arvensis ô $q$, fq; Prosopid.: Prosopis illinoensis $q$, pygmaea $0^{\lambda}$.

Other Hymenoptera (29)—Sphec.: Ammobia ichneumonea, pennsylvanica, Isodontia apicalis, Sphex gracilis, nigricans, vulgaris; Scoli.: Elis interrupta, 5-cincta, Scolia bicincta; Philanth.: Cerceris fumipennis, Eucerceris, Philanthus punctatus fq, ventilabris; Vesp.: Polistes rubiginosus, variatus; Eumen.: Leionotus arvensis $\mathrm{fq}$, dorsalis, foraminatus fq, 4-sectus; Crabron.: Anacrabro, Lindenius errans, Solenius; Oxybel.: Notoglossa americana, frontalis, Oxybelus packardii ; Larr.: Tachytes distinctus ; Pompil.: Arachnoproctonus, Poecilopompilus navus; Chalcid.: Leucospis. 
Diptera (22) -Stratiomy.: Stratiomyia meigenii; Syrph.: Allograpta, Mesogramma marginata, Syritta fq; Conop.: Conops brachyrrynehus, Oneonyia, Physoeephala tibialis, Zodion nanellum; Tachin.: Archytas analis fy, Limnatemyia fy, Ocyptera lata, Phorantha purpuraseens, Senotainia trilineata fq. Trichopoda pennipes, Triehophora; Sarcophag.: Helieobia helieis, Sareophaga cimbieis; Muse.: Compsomyia fy, Lueilia sylvarum, Musear, Psendopyrellia $\mathrm{fq}$; Anthomy.: Calythea.

Rhus glabra (7: 111-12).-Blooms May $2 \overline{7}-\mathrm{July} 7 ; 114$ visitors observed, June 5-July 7; Mis, Y.

On staminate flowers (57):-

Long-tongued Bees (4)-Bomb.: Bombias separatus $\zeta$, se; Slelil.: Mierostelis $q$; Meguchil.: 'Trypetini: Veotrypetes truncatus $\delta َ$, se, fq; Coelioxyini: Coelioxys sayi $q$.

Short-tongued Bees (11) - Halict.: Curtisapis fuscipennis ${ }_{7}$ se,

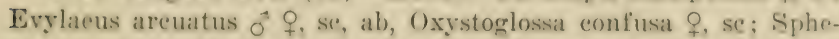
codini: Spheeodes arvensis of Prosopid.: Prosopis illinoensis of, ziziae of + , sf; Collet.: Colletes aestivalis of 을 nudus $\delta$; Andren.: Andrena platyparia $q$, se, Opandrena eressonii $q$, se, Trachandrena nuda $\delta$.

Other Hymenoptera (10)-Bombic:: Bicyrtes ventralis; Philunth.: Cerceris compar, fumipennis, insolita, Philanthus punetatus; Eumen.: Aneistrocerus eampestris, Leionotus arvensis, Monobia, Zethus; Crabron.: Solenius.

Diptera (29)-Stratiomy.: Stratiomyia apienla, meigenii. quaternaria; Syrph.: Baceha fuseipennis, Ceria, Eristalis dimidiatus, flavipes, tenax, transversus, Mallota cimbiciformis, posticata, Mesogramma geminata, Orthoneura nitida, Sphatephoria, Sphegina rufiventris, Syrphus americanus, ribesii; Empid.: Parempis fq, Bombyli.: Anthrax alternata, sinuosa f, Bombylius atriceps; Conop.: Conops brachyrrhynchus, Zodion fulvifrons, nanellum ; Tuchin.: Hypostena variabilis, Siphona; Muse.: Lneilia carsar, Morellia; Anthomy.: Limnophora.

Lepidoptera (3)-Nymphal.: Neonympha; Lycum.: Oyaniris. Theela ealanus.

On staminate and pistillate flowers (18):-

Long-tongued Bees (1)-Ceratin.: Ceratina 오. se.

Short-tongned bees (10)-Halict.: Agapostemon radiatus o, se, viridulus f. se, Chloralictus pilosus f, se, pruinusus if i, se,

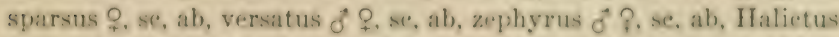
Jermuxii of 9 . se. fq; Collet.: Colletes eulophi of 8, ab; . Indren.: Opandrena serotina $\delta$, se, fq. 
Other Hymenoptera (3)-Sphec.: Sphex vulgaris; Philanth.: Cerceris robertsonii fq; Oxybel.: Notoglossa americana.

Diptera (4)-Syrph: Allograpta, Mesogramma marginata fq; Conop.: Physocephala tibialis; Tachin.: Ocyptera tenuis.

On pistillate flowers (39):-

Long-tongued Bees (3)-Ap.: Apis ab; Bomb.: Bombias auricomus + ; Ceratin.: Zaodontomerus $\delta^{7}$.

Short-tongued Bees (7, q)-Halict.: Chloralictus tegularis, Halictus parallelus, Oxystoglossa similis, Seladonia; Prosopid.: Proșopis pygmaea; Collet.: Colletes willistonii ab; Andren.: Trachandrena crataegi.

Other Hymenoptera (10)-Sphec.: Isodontia apicalis, Priononyx thomae, Sphex procera; Philanth.: Cerceris compacta fq; Vesp.: Polistes pallipes; Eumen.: Leionotus anormis fq; Oxybel.: Notoglossa frontalis; Pemphredon.: Mimesa; Pompil.: Pompiloides marginatus; Bracon.: Vipio.

Diptera (18)—Syrph.: Syritta; Tachin.: Archytas analis ab, aterrima, Belvosia bifasciata, Cyphocera, Pachyophthalmus floridensis, Peleteria, Spallanzania; Sarcophag.: Helicobia helicis, 4-setosa, Sarcophaga cimbicis, hunteri, sinuata; Musc.: Lucilia sylvarum, Pseudopyrellia, Stomoxys; Anthomy.: Phorbia acra, platura.

Coleoptera (1)-Mordell.: Mordella melaena.

June 17, 1913, the following 17 species and 51 individuals were taken on pistillate flowers of Rhus glabra:

Long-tongued Bees (3:19)—Bomb.: Bombias auricomus 1; Ceratin.: Ceratina 17, Zaodontomerus 1.

Short-tongued Bees $(10: 28)$-Halict.: Agapostemon radiatus 2 , viridulus 1 , Chloralictus pilosus 1 , sparsus 9, tegularis 1 , versatus 6, zephyrus 2, Oxystoglossa similis 2; Prosopid.: Prosopis pygmaea 1; Andren.: Opandrena serotina 3.

Other Hymenoptera $(1: 1)$-Sphec.: Sphex procera 1.

Diptera (3:3)-Syrph.: Allograpta 1, Syritta 1; Tachin.: Peleteria 1.

Rhus toxicodendron.-Blooms May 18-June 29; 1 visitor observed, June 8; Mis, Y.

Short-tongued Bees (1)-Andren.: Trachandrena crataegi + , sc.

\section{ANONACEAE}

Asimina triloba (16: 154-5).-Blooms April 10-May 27; 8 visitors observed, May $5 ; \mathrm{Mi}, \mathrm{R}$. 
Diptera (A) -syrph.: Syrphus amerieanus; sarcophay.: Cynomyia fq, Helieobia helieis, t-setosa, Sareophaga sinuata; Musc.: Phormia regina; Inthom!l.: Phorbia platuma; Scutophag.: Scatophaga.

\section{APOCYNACEAE}

Apocynum androsaemifolium.-Bloums May 10-July 28; 12 visitors observed, July 16,$25 ;$ Mis, W.

Long-tongued lioes (1)-Comad.: Centrias americanus \&. mp.

short-tongued Bees (5, no pul.) - Halict.: Chloralietus versatus +. zephyrus q. Oxystoglosia confusa of. similis of f; Proseprid.: Prosopis sayi $q$.

Other IIymenoptera (1)-Sphec.: Sphex vulgaris, no pol.

Diptera (5, n)-Tarhin.: Linnatemyia, Triehophlora: Musr.: Graphomyia, Lucilia sylvarum; Anthomy.: Phorbia acra.

Apocynum cannabinum (4:70).-Blooms May 18-August 12; 48 visitors observed, June $1-$ July 17 ; Mis, W.

With pollinia (9):-

Long-tongued Bees (3) - Ap.: Apis $1 p$; Nomud.: Centrias amerieanus $\delta q, \mathrm{fq}, \mathrm{mp}, \mathrm{lp}$; Holonomada affabilis $\delta, \mathrm{lp}$.

Short-tongued Bees $(3, \mathrm{mp})$ - Halict. (q) : Agapostemon radiatus, Oxystoglossa coufusa; Collet.: Colletes produetus of, type.

Other Hymenoptera (3) - Sphec.: Isodontia apicalis t; Bembic.: Bieyrtes ventralis mp ; Eumen.: Leionotus foraminatus t.

Without pollinia (39) :-

Long-tongued Bees (3)-Stelid.: Microstelis + ; Megachil.: Megachilini: Chelostomoides $q$; Coelioxyini: Coelioxys s-dentata c우.

Short-tongued Bees (6)-Malict.: Augoehlora viridula ${ }^{\circ}$. Chloralietus versatus $\delta$ $q$. Seladonia ${ }_{+}$; Prosopid.: Prosopis ziziae 7; Collet.: Colletes aestivalis oै; Macropid.: Macropis d. fq.

Other Hymenoptera (4) - Sphec.: Priononyx atrata, thomae, Sphex vulgaris; Larr.: Astatus unicolor.

Diptera (21, n)-Mycetophil.: Sciara atrata; Syrph.: Allograpta, Eristalis dimidiatus, Helophilus laetus, Sphaerophoria, Tropilia mamillata, quadrata; Empid.: Parempis; Bumbyli.: Anthrax alternata, Bombylius atriceps; Tachin.: Archytas analis. f'istogaster oceidua, Linnaemyia, Oeyptera truuis, Sjallanzania, Trichophora; Surcophag.: Helienbia helieis: Muse : Compsomyia, Phormia regina; Anthomy.: Limnophora, Phorbia acra.

Lepidoptrra (2, n)-Nymphal.: Argyunis; Lyeacn.: Theda calanus. 
Coleoptera (1)-Scarabae.: Trichius piger $\mathrm{n}$.

Hemiptera $(2, \mathrm{n})$-Caps.: Lygus ; Lygae.: Lygaeus.

\section{Araceae}

Arisaema triphyllum.-Blooms April 27-July 1; 1 visitor observed, May 11, also twenty specimens belonging to about seven species of Nematocera; Mi, Y.

Hemiptera (1) -Nab.: Coriscus.

\section{Aristolochiaceae}

Aristolochia tomentosa.-Blooms May 9-June 24; 5 visitors observed, May 15-June 13; Mi, Y.

Diptera (4)-Chironom.: Ceratopogon websteri; Mycetophil.: Sciara atrata, vulgaris; Phor.: Phora.

Coleoptera (1)-Melandry.: Canifa.

\section{AsClepiadaceae}

According to the situation of the pollinia the insects carrying them are marked: $\mathrm{c}=$ claws, $\mathrm{emp}=$ empodium, $\mathrm{f}=$ face, $\mathrm{g}=$ glossa, $\mathrm{h}=$ tarsal hairs, $\mathrm{l}=$ labrum, $\mathrm{lp}=$ labial palpi, $\mathrm{mp}=$ maxillary palpi, $\mathrm{p}=$ pulvillus, $\mathrm{sp}=$ tibial spur, $\mathrm{t}=$ proboseis, usually palpi, $\mathrm{vs}=$ hairs of ventral surface.

Acerates floridana $(1: 245-7 ; 13: 576-7)$.-Blooms June 24August 16; 18 visitors observed, July 5-August 16; Mas, W.

With pollinia (10):-

Long-tongued Bees (6:21)-Ap.: Apis vs; Bomb.: Bombias

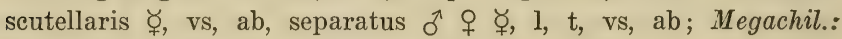
Megachile generosa + , vs, mendica + , vs.

Other Hymenoptera (4)-Sphec.: Priononyx atrata vs; Bembic.: Bembix nubillipennis 1 , ab; Philanth.: Cerceris bicornuta vs, f, t; Pompil.: Priocnemoides unifasciatus vs, $t$.

Coleoptera (1)-Scarabae.: Trichius piger vs.

Without pollinia (8):-

Long-tongued Bees (1)-Megachil.: Megachile brevis $q$.

Other Hymenoptera (5)-Scoli.: Elis 5-cincta; Philanth.: Cerceris compar; Vesp.: Polistes variatus ; Eumen.: Leionotus arvensis; Bracon.: Orgilus.

Lepidoptera (2)—Lycaen.: Chrysophanus ; Ctenuch.: Scepsis fq.

Acerates viridiflora (1:247-8).-Blooms June 8-July 29; 5 visitors observed, June 14-July 29 ; Mas, Y.

Long-tongued Bees $(3, \mathrm{~h})$-Bomb.: Bombias scutellaris $\Varangle$, separatus $\not{\gamma}, \mathrm{fq} ;$ Eucer.: Anthedon $\sigma^{\top}$. 
Other Hymenoptera (2)-Larr.: Tachytes mandibularis h; Eumen.: Leionotus arvensis, no pollinia.

Asclepias incarnata $(1: 209 ; 13: 571-2)$.- Blooms July 5-September 2 ; 112 visitors observed, July 22-September 2 ; Pol (Mas), R.

With pollinia $(88)$ :-

Long-tongued Bees (12)-Ap.: Apis h, t, ab, one dead; lBomb.:

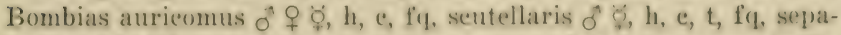

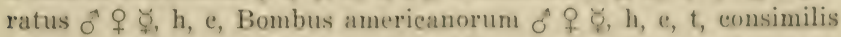

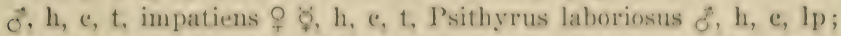
Eucer.: Epimelissodes atripes o, h, Melissodes eomptoirles $\hat{o}$, h, trinodis q, h ; Emphor.: Melitoma ơ, h.

Short-tongued Bees (6) - Ilulict.: Augochlora fervida of $q, \mathrm{~h}$. fq. Curtisapis eoriacea $\hat{\delta}, h, t$. Halictus lerouxii $\hat{d}, p$, Oxystoglossa similis 8 , t. Seladonia $q . h$; Collet.: Colletes latitarsis $q, \mathrm{~h}, \mathrm{e}, \mathrm{t}$, dead.

Other Ilymenoptera (42)-Sphec.: Ammobia iehneumonea h, nucla h, pennsylvanica h, e.t, fq. Isodontia apiealis h, macroeephala h, Priononyx atrata h, fq. thomate h, fq. Seeliphron h, one dead; Bembic. (h) : Bembix nubillipeunis f(1, spinolae, Megastizus, Sphecius fq, Śtictia; ścoli.: Elis interrupta h, t, obscura h, j-eincta h, e, t. fq. Seolia bieineta h; Philanth.: Cereeris bieornuta h, e, t, fq, elypeata h, e, eompacta h, e, finitima t, fumipennis p; Vesp.: Polistes pallipes h, e, rubiginosus h, c, variatus h, e, Vespulat euneata h. e, t, fy. germanica h. maeulata h, vidua h, e, t; Eumen.: Leionotus arvensis h. e. t, ab; Crabron.: Protothyreopus rufifemur c; Orylbel.: Oxybelus packardii e, $\mathrm{t}$; Larr.: Tachytes aurulentus h, distinctus h, mandibularis h, t, fq, pepticus h; Nysson.: Psendoplisus phaleratus h; Pompil. (h) : Ageniella aceepta, Arachnoproctonus, Episyron, Lophopompilus atrox, Prioenemoides unifasciatus.

Diptera (8)-Stratiomy.: Stratiomyia meigenii e; Mirla.: Midas clavatus h, t, tibialis p; Conop.: Comops xanthopareus p, fq. Physoeephala tibialis h; Tachin.: Cistogaster oecidua t, n, P'anzeria c, Trichopoda lanipes h.

Lepidoptera (15)-Nymphal.: Anosia h, Argynnis h, Basilarehia arehippus h, Hypatus h, Speyeria h, Vanessa atalanta h, e; Papilion.: Euphoeades h, Eurymus h, Heraclides h. Jasoniades h, e, Laertias h, I'apilio h, e, Pieris h, e; Hesperi.: Achalarus h, Limochores taumas $\mathrm{h}$.

Coleoptera (3) - Lampyr.: Chauliognathus pumsylvanicus h, e, t, ab; Scarabue.: Euphoria sepulchralis, h, c, t. ab. Trichius piger h.

Hemiptera (2)-Lygae.: Lygaeus h, Oncopeltus h.

Without pollinia (24) :-

Birds (1)-Trochil.: Trochilus n. 
Long-tongued Bees (1)-Megachil.: Megachile brevis 01 ㅇ.

Short-tongued Bees (1)-Halict.: Chloralictus versatus 0 , n.

Other Hymenoptera (3)-Eumen.: Eumenes fraternus; Oxybel.: Notoglossa americana; Pompil.: Priocnemoides fulvicornis.

Diptera (9)-Bombyli.: Sparnopolius $\mathrm{n}$; Conop.: Conops brachyrrhynchus n, Stylogaster biannulata $\mathrm{n}$; Tachin.: Gymnoprosopa n, Ennyomma globosa n, Masiphya n, Opsidia n, Phasiocliata $n$, Spallanzania.

Lepidoptera (6)-Nymphal.: Phyciodes; Hesperi.: Epargyreus, Euphyes verna, Pholisora catullus, Polites; Ctenuch.: Scepsis.

Coleoptera (3, n)-Buprest.: Acmaeodera pulchella; Cerambyc. Tetraopes gn; Melo.: Epicauta vittata gn, fq.

Asclepias purpurascens $(1: 213 ; 13: 576)$.-Blooms May 27July 15; 36 visitors observed, June 2-28; Mas, R.

With pollinia (14) :-

Long-tongued Bees (5)-Ap.: Apis h; Megachil.: Megachile addenda $q, \mathrm{~h}$, mendica $\delta$, h; Eucer.: Tetralonia rosae $q, \mathrm{~h}$; Anthophor.: Anthemoessa $\delta^{\wedge}+\mathrm{h}, \mathrm{p}, \mathrm{fq}$.

Diptera (2)-Conop.: Physocephala tibialis h; Tachin.: Spallanzania $\mathrm{p}$.

Lepidoptera (5, h)-Nymphal.: Argynnis fq, Euphydryas, Speyeria, Vanessa atalanta, huntera; Hesperi.: Thorybes pylades.

Hemiptera (1)-Lygae.: Oncopeltus h.

Without pollinia (28):-

Birds (1) - Trochil.: Trochilus n.

Long-tongued Bees (3, 우)-Bomb.: Bombias separatus, Bombus americanorum, consimilis; Anthophor.: Anthophora.

Short-tongued Bees (4, $q, \mathrm{n})$-Halict.: Augochlora fervida, Chloralictus versatus, Evylaeus pectoralis, Oxystoglossa confusa, similis.

Other Hymenoptera (1)—Sphec.: Priononyx atrata.

Lepidoptera (13)-Nymphal.: Anosia; Lycaen.: Chrysophanus, Everes, Thecla calanus, sylvinus; Papilion.: Euphoeades, Eurymus, Heraclides, Jasoniades, Laertias; Hesperi.: Euphyes verna, Limochores manataaqua, taumas, Pamphila hobomok, Polites.

Coleoptera (1)-Cerambyc.: Tetraopes gn, $\mathrm{n}$.

June 19-28, the following 22 species and 58 individuals were taken on the flowers:-

With pollinia $(4: 14)$ :-

Long-tongued Bees (2:5)-Ap.: Apis 4; Anthophor.: Anthemoessa ${ }^{\prime}, 1$.

Lepidoptera (2:9)-Nymphal.: Argynnis 6, Vanessa huntera 3. 
Without pollinia $(20: 44)$ :-

Birds (1:1)-Trochil.: 'Troehilus 1.

Long-tongued Bees $(3: 9, q)$-Bumb.: Bombias separatus 1; Anthophor.: Anthemoessa 7, Anthophora 1.

Short-tongued Bees (4:7, 7 )-Uuliet.: Augochlora fervida 2; ('hloralietus versatus 1, Evylaeus pectoralis 3. Oxystoglossa similis 1 .

Lepidoptera (9: 2:3)-Nymphal.: Argynnis 1; Papilion.: Euphoeades 3. Ieraclides 1, Jasoniades 5. Laertias $2 ;$ IJesprri.: Euplyyes verna 1, Limochores taumas 2, Polites 2, Thorylos pylades 5 .

Diptera (2:3)-Tuchin.: Trichophora 2, Spallanzania 1.

Hemiptera (1:1)-Lygae.: Oneopeltus 1.

Asclepias sullivantii $(1: 210-12 ; 13: 571-5)$.-13lnoms Jume 10-Aurust 23; 90 visitors observed. June 19-August 20; Mas, R.

With pollinia (25):-

Long-tongued Bees (11) - Bomb.: Bombias aurieomus of ఫ̈. e, p),

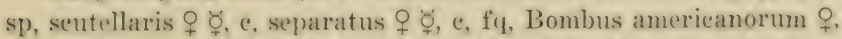
h. e, P'sithyrus variabilis $q$, e, sp, h; Megachil.: Megachile petulans

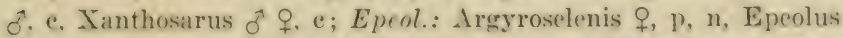
hifasciatus ô. 1, n; Eucer.: Florilegus ô, 1, Melissodes coreopsis f, p.

Other Hymenoptera (4)-Sphec.: Priononyx thomae h, Seeliphron e; Bembic.: Bembix nubillipennis e Iehneumon.: Ichneumon flavizonatus, on antenna, $\mathrm{n}$.

Diptera (3)-Stratiomy.: Stratiomyia meigenii c, emp ; Connp.: Physocephala tibialis p, Zodion obliquefasciatum p.

Lepidoptera ( 7$)$-Nymphal.: Anosia e, h, sp, Areymis e; Lyear n.: Strymon h, n; Papilion.: Eurymus c, Papilio c; IIesperi.: Epargyreus c, Limochores manataaqua c.

Without pollinia, (46) :-

Birds (1)-Trochil.: T'rochilus.

Long-tongued Bews (4)-Cratin.: Ceratina 9: Megmhil.: Megachilini: Megachile generosa of o. ab, mendiea $\hat{f}$ : Coelinxyini : Coelioxys 8-dentata $\sigma^{\star}$ 우, fq.

Short-tonewed Bees (9) - Mulict.: Aneochlora viridula?, Chloralictus pilosus 9. sparsus o. tegularis of. versatus of ?. Oxystogrlussa

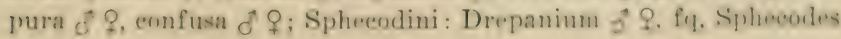
arvensis .

Ofher IIymenoptera (12)-Sphec.: Priononys atrata: Rembie.: Bembix spinolar, Microbembex fy; Scoli.: Elis n-eineta: Corer.: Cereeris elypeata, finitima; Philenth.: I'hilanthus punctatus; 
Eumen.: Leionotus arvensis; Crabron.: Protothyreopus rufifemur; Oxybel.: Notoglossa americana; Larr.: Lyroda, Tachytes mandibularis fq.

Diptera (7)-Syrph.: Tropidia quadrata; Tachin.: Chaetogaedia analis (acroglossoides type), Linnaemyia, Phorantha humeralis, Senotainia rubriventris, trilineata; Sarcophag.: Helicobia helicis.

Lepidoptera (10)-Nymphal.: Phyciodes, Vanessa atalanta; Lycaen.: Chrysophanus, Everes, Uranotes; Hesperi.: Euphyes metacomet, Limochores taumas, Thorybes pylades; Sphing.: Hemaris axillaris ; Sesi.: Sesia 6-fasciata.

Coleoptera (1)-Curculion.: Centrinus scutellum album fq.

Hemiptera (2)-Caps.: Calocoris; Pentatom.: Podisus, pred.

The following insects were found entrapped and dead on the flowers (19):

Long-tongued Bees (6)-Ap.: Apis 671 ఫ, 1 ô; Megachil.: Anthemois $\sigma^{\lambda} q, \mathrm{c}, \mathrm{p}$, Megachile addenda $\delta$, brevis $\sigma^{\lambda} q$, c, Sayapis pugnata $\sigma^{\wedge}$; Eucer.: Peponapis $\sigma^{\top}$.

Short-tongued Bees $\left(3, \delta^{1}\right)$-Halict.: Halictus lerouxii c, p, Odontalictus, Seladonia.

Other Hymenoptera (3)-Sphec.: Isodontia apicalis h, c, Sphex pictipennis c; Larr.: Astatus bicolor.

Diptera (4)—Syrph.: Eristalis aeneus c, Syritta; Bombyli.: Sparnopolius; Musc.: Pseudopyrellia.

Lepidoptera (2)-Hesperi.: Polites p ; Ctenuch.: Scepsis.

Coleoptera (1)—Scarabae.: Trichius piger c.

The following 22 species and 140 individuals were taken on the flowers, June 19, 20, 1911:

With pollinia $(6: 9)$ :-

Long-tongued Bees (2:5)—Bomb.: Bombus americanorum 3; Megachil.: Xanthosarus 2.

Diptera (1:1)-Conop.: Physocephala tibialis 1.

Lepidoptera (3:3)-Nymphal.: Argynnis 1; Hesperi.: Epargyreus 1 , Limochores manataaqua 1.

Without pollinia $(20: 131)$ :-

Long-tongued Bees (4:22)-Megachil.: Megachile brevis 18, generosa 1, mendica 1, Xanthosarus 2.

Short-tongued Bees (4:86)-Halict.: Chloralictus pilosus 1, tegularis 1 , versatus 83 , Oxystoglossa confusa 1 .

Other Hymenoptera (4:7)-Sphec.: Priononyx atrata 1; Bembic.: Bembix nubillipennis 1, spinolae 1; Larr.: Tachytes mandibularis 4 . 
Lepidoptera (8:16)-Nymphal.: Argynnis 2. Phycionles 1; Lycaen.: Uranotes 1; II speri.: Epargyreus 1, Euphyes metacomet 1, Limolores manataayua 8 , Thorybes pylades 1 ; Sphing.: Hemaris axillaris 1 .

Asclepias syriaca $(1: 20 !)-10: 13: 5 \%-4)$. Blooms Jume 1August 15; 99 visitors ohserved. Jume 7 -. Iumust 7 ; Mas, R.

With pollinia $(60)$ :-

Loug-tongued Bees (15)-Bomb.: liombias separatus $q \not \phi, t$; Megachil.: Megachilini: Megachile generosia of, $t$, petulans \&, 1 ; Corlinxyini: Coelioxys S-rlentata oै o, e, n: Epcol.: Epeolus bifasciatus $\delta$, p, n, Triepeolus remigatus of, p; Eucr.: Florilecrus

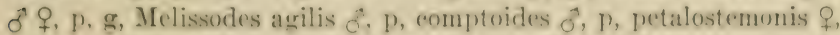
p. Peponapis ô $q, h, p, t, f q$, T'etralonia dilecta $q, \mathrm{p}$, rosae $\&, \mathrm{~h}$, Xenoglossa + , p; Anthophor.: Anthomoessa ơ, p.

Short-tongued Bees (6)-Ilalict. (n) : Agapostemon radiatus of, p. Halietus lerouxii of, p, Seladonia o, e; Sphecorlini : Proteraner $\delta$, p; Collet.: Colletes latitarsis $\sigma, \mathrm{p}, \mathrm{lp}$, nudus $\mathrm{q}^{7} \mathrm{p}$.

Other Hymenoptera (12) - Sphec: Ammobia ichneumenea h, Primonyx atrata $\mathrm{p}, \mathrm{fq}$, thomat $\mathrm{h}$; Bembie.: Bembix nubillipennis h. Stietia p; Scoli.: Campsomeris h. «. Elis obsirura p, 5-eineta h, Seolia bieineta h: Philanth.: Cerceris bieornuta h; Eumen.: lecimotus arvensis h; larr.: Tachytes mandibularis h.

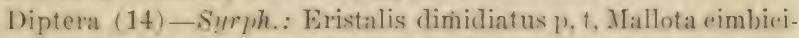
formis p. t, illinoensis p. Syritta p. Tropidia mamillata p, quadrata h: Mida.: Midas elavatus t; Conop.: Physuceplaala tezana p; Tuhin.: Arehytas analis p, Belvosia bifasciata p, mifasciata $p$. Limaemyia p, Trichopoda plumipes p, $t$; Anthom!. : IIyctodosia p.

T.epidoptera (8)-Nymphal.: Anosia h, Argymis h, Euvanessa h, e, Polygonia interrogationis h, t, Vanessa atalanta h; IIesperi.: Arhalarus h: Sphing.: Choerorampa h: Ctenuch.: Seepsis h.

Coleoptera (1)-Lampyr.: Photinus h.

Iremiptera (4)-Lygae.: Lygaeus h, p, e. Oncopeltus h: T'entutom. : Euschistus variolarius h, l'odisus p, pred. n.

Without pollinia (31) :-

Long-tungued Bees (4) - Bomb.: Iombias aurieomus 8 . Iombus amerieannrum \&: Megachil.: Megachile brevis ?: Eucr.: Epimelissodes obliqua $\sigma^{\top}$.

Short-tonerued Bues (1)--Ifalicl.: Augochlora viridula ?. n.

Other IIymenoptera (2) - Sphec.: Seeliphron; Philanth.: Cerceris elypeata.

Diptera (5, n)-Culic.: Culex stimulans: Stratiomy.: Odontomyia cincta; Bombyli.: Inthrax sinuosa: Conop.: Stylneaster. biannulata ; Tachin.: Cistogaster oceidua. 
Lepidoptera (13)-Nymphal.: Basilarchia archippus, Speyeria; Lycaen.: Chrysophanus, Thecla calanus; Papilion.: Eurymus, Laertias, Papilio; Hesperi.: Epargyreus, Limochores taumas, Pholisora catullus, Polites; Pyraust.: Loxostege.

Coleoptera (4, n)-Cerambyc.: Tetraopes gn; Elater.: Melanotus gn; Scarabae.: Macrodactylus gn, Trichius piger.

Hemiptera $(2, \mathrm{n})$-Caps.: Lygus; Reduvi.: Sinea.

The following visitors were found dead on the flowers (8):-

Long-tongued Bees (1)-Ap.: Apis ab, p, t.

Diptera (4)-Tachin.: Spallanzania p; Sarcophag.: Sarcophaga cimbicis p; Musc.: Pseudopyrellia, Stomoxys.

Lepidoptera (3)-Arcti.: Callimorpha p; Noctu.: Drasteria erechtea h, Agrotis.

Asclepias tuberosa $(1: 212-13 ; 13: 575-6)$.-Blooms June 6September 5; 29 visitors observed, June 14-August 17; Mas, Y.

With pollinia (19):-

Long-tongued Bees (5)-Ap.: Apis h; Stelid.: Anthidium cognatum $\sigma^{\lambda}$, h; Megachil.: Coelioxys 8-dentata $\uparrow$, h, c; Eucer.: Melissodes petalostemonis $q, \mathrm{~h}$, variabilis $\sigma^{\lambda}, \mathrm{h}$.

Short-tongued Bees (1)-Halict.: Augochlora fervida o, c, fq.

Other Hymneoptera (4, h)—Sphec.: Ammobia ichneumonea, Priononyx thomae, Sphex nigricans, procera ab.

Diptera (1) - Tachin.: Spallanzania h.

Lepidoptera $(8, \mathrm{~h})$-Nymphal.: Argynnis, Speyeria; Papilion.: Euphoeades, Eurymus, Laertias, Papilio, Pontia; Ctenuch.: Seepsis.

Without pollinia (10):-

Birds (1)-Trochil.: Trochilus n.

Long-tongued Bees (2)-Megachit.: Cyphopyga $q$, Megachile brevis $\sigma^{\pi}$ ㅇ.

Other Hymenoptera (1)—Sphec.: Priononyx atrata.

Lepidoptera (6)-Nymphal.: Anosia, Phyciodes; Lycaen.: Chrysophanus, Everes, Strymon; Papilion.: Jasoniades.

Asclepias verticillata $(1: 207-8 ; 13: 569-71)$.-Blooms June 26 -September 4 ; 131 visitors observed, June 26-August 21; Pol, W.

With pollinia (71) :-

Long-tongued Bees (10)-Ap.: Apis h;Bomb.: Bombias separatus $\sigma^{\top} q \not{q}, \mathrm{~h}, \mathrm{fq}$, Bombus impatiens $\not, \mathrm{h}$, Psithyrus laboriosus; ㅇ, h; Ceratin.: Ceratina + , h, c, p, fq ; Megachil.: Trypetini : Neotrypetes barbatus $q$, p, type; Megachilini: Megachile brevis 0 , h, t, fq; Coelioxyini : Coelioxys 8-dentata $\sigma^{\lambda}, \mathrm{h} ;$ Epeol.: Triepeolus concolor $\sigma^{\uparrow}, \mathrm{h}$; Eucer.: Melissodes bimaculata $\delta^{\lambda}+, \mathrm{h}, \mathrm{t}, \mathrm{fq}$. 
Short-tongued Bees (10)-Hulict.: Augrochlora fervida of f, h, Chloralietus pilosus $q, \mathrm{mp}$, versatus of $q, \mathrm{p}$, Curtisapis forbesii $\mathcal{o}^{\text {, }}$ Ip, Evylaeus pectoralis,$+ \mathrm{h}$, Halictus lerouxii of $q, \mathrm{~h}, \mathrm{c}, \mathrm{t}$, Seladonia $o^{2} q, \mathrm{~h}, \mathrm{p}, \mathrm{ab}$; Spheeodini : Machaeris stygia $q, \mathrm{~h}$, Spheeodes arvensis $\mathrm{o}^{2}, \mathrm{~h}, \mathrm{t} ;$ Prosopid.: Prosopis ziziae $q, \mathrm{p}$.

Other Hymenoptera (32)-Sphec. (h) : Ammobia iehneumonea fq, pennsylvaniea, Isodontia apicalis, Priononyx thomae; Bembic: Bembix numbilipennis h; Scoli.: Elis obscura h, 5-eineta h, t, fil, Seolia bicineta h; Philunth.: Cerceris bicornuta h, ab, elypeata h, e, fq, compacta h, e, fq, raui h, fulvipes h, robertsonii h; Vesp.: Polistes variatus h; Eumen.: Eumenes frateruus h, e, t, Leionotus anormis $h, t, a b$, arvensis $h, f(q$, foraminatus $h$; Crabron.: Anaerabo e, p, Protothyreopus rufifemur h, p, fq; Larr. (h) : Astatus unicolor, Tachytes distinetus, elongatus, mandibularis, peptieus fq; Pompil. (h) : Anoplius illinoensis, Batizonus, Lophopompilus philadelphieus, Prioenemoides fulvicornis, terminatus; Tenthredin.: Hylotoma humeralis $\mathrm{h}$.

Diptera (16)—Syrph.: Eristalis latifrons h, Syritta e, Tropidia quadrata h; Mida.: Midas clavatus h ; Conop.: Physocephala texana $\mathrm{h}$, tibialis $\mathrm{h}$; Tachin.: Arehytas analis h, Clytiomyia h, t, Linnaemyia h, t, Oeyptera tenuis h, t, Spallanzania h, e, p, t, Xanthomelana $\mathrm{t}, \mathrm{n}$, Senotainia trilineata $\mathrm{t}, \mathrm{f}(\mathrm{l}, \mathrm{n} ;$ M use.: Compsomyia $\mathrm{e}, \mathrm{t}$, Phormia regina c, Pseudopyrellia h, e, t.

Lepidoptera (3, h)-Nymphal.: Anosia ; Hesperi.: Pholisora hayhurstii, Polites.

Without pollinia (60) :-

Long-tongued Bees (1)-Bomb.: Bombus americanorum $\Varangle$.

Short-tongued Bees (4)-IIalic.: Agapostemon radiatus $q$, Chloralictus albipennis $\hat{\sigma}$, sparsus $q$; Sphecodini: Sphecodium cressonii $\sigma^{\top}$.

Other Hymenoptera (9)-Sphec.: Priononyx atrata, Seeliphron, Sphex nigrieans; Scoli.: Elis interrupta ; Philanth.: Cereeris finitima; Crabron.: Solenius; Oxybel.: Notoglossa frontalis; Formici.: Formica rufibarbis; Chrysid.: Holopyra.

Diptera (29 n)-Syrph.: Allograpta, Eristalis transversus, Mesogramma marginata, polita, Orthoneura nitida, Paragus bicolor, Sphaerophoria ; Empid.: Parempis; Bombyli.: Exoprosopa fasciata, Systoechus; Conop.: Conops brachyrrhynchus; Tachin.: Cistogaster oceidua, Exorista confinis, Gymnoprosopa, Ennyomma globosa, Paradidyma, Phorantha purpurascens, Senotainia ruhriventris, Siphoplagia anomala, flacedirostris, Trichopoda pennipes, Triehophora; Sarcophag.: Helicobia helicis, Sarcophaga sinuata; 
Musc.: Graphomyia, Lucilia sylvarum; Anthomy.: Calythea, Limnophora; Seps.: Sepsis.

Lepidoptera (13)-Nymphal.: Argynnis, Phyciodes, Vanessa atalanta; Lycaen.: Chrysophanus, Everes; Papilion.: Eurymus, Heraclides, Pieris; Hesperi.: Achalarus, Euphyes verna, Limochores taumas, Pholisora catullus; Ctenuch.: Scepsis.

Coleoptera (3n)-Cerambyc.: Typocerus sinuatus; Rhipiphor.: Rhipiphorus limbatus; Scarabae.: Trichius piger.

Hemiptera (1)-Phymat.: Phymata, pred, n.

Gonolobus laevis (8:108-10)-Blooms July 12-August 23; 22 visitors observed, July 14-August 23; Mis, W.

With pollinia on proboscides (14):-

Long-tongued Bees (1)-Eucer.: Epimelissodes atripes $q$.

Short-tongued Bees (4, o $q$ )-Chloralictus sparsus ab, tegularis, versatus ab, zephyrus ab.

Other Hymenoptera (4)-Sphec.: Ammobia nuda; Scoli.: Elis interrupta, 5-cincta fq; Vesp.: Polistes variatus.

Diptera (5)-Bombyli.: Anthrax fulvohirta; Conop.: Conops xanthopareus; Tachin.: Alophora aeneoventris, Panzeria; Musc.: Lucilia sericata.

Without pollinia (8) :-

Short-tongued Bees (6)-Halict.: Augochlora viridula ô $q$, Chloralictus illinoensis $\delta$, Paralictus cephalicus $q$, platyparius $q$; Prosopid.: Prosopis illinoensis + , pygmaea $\delta^{7}$.

Other Hymenoptera (1)-Eumen.: Leionotus ziziae.

Diptera (1)-Empid.: Parempis n.

\section{Balsaminaceae}

Impatiens biflora (2:300-1)-Blooms June 19-September 20; 11 visitors observed, July 30-September 12 ; Ma, R.

Birds (1)-Trochil.: Trochilus fq.

Long-tongued Bees (6)-Ap.: Apis sc; Bomb.: Bombus ameri-

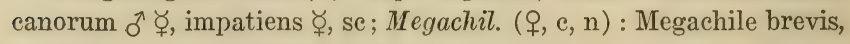
mendica; Eucer.: Melissodes bimaculata ${ }^{\circ}$.

Short-tongued Bees $(2, q, \mathrm{c}, \mathrm{n})$-Halict.: Chloralictus versatus, Oxystoglossa confusa.

Lepidoptera (1)-Papilion.: Euphoeades.

Coleoptera (1)-Chrysomel.: Diabrotica 12-punctata gn, $\mathrm{n}$.

Impatiens pallida (2:301-2)-Blooms June 11-September 17; 6 visitors observed, July 23-September 17; Ma, Y.

Birds (1)_Trochil.: Trochilus. 
Long-tongued Bers (4)-Bomb.: Bombus amerieanorum of ab, impatiens $\varnothing$, se, ab; Megachil.: Megachile brevis $7, r, n$.

Short-tongued liees (1)-Muliet.: Chloraliefus versatus o, u.

Diptera (1)-Syrph.: Rhingia sf, $n$.

\section{Berberidaceaf}

Caulophyllum thalictroides $(9: 154-5)$.-Blooms April 23May 7 ; 29 visitors observed, April 2:-May 3 ; Mi, Y.

Long-tongued Bees $(3, f)$-Bomb.: Bombias separatus, Bombus impatiens, ridingsii.

Short-tongued Bees (\&, o)--Halict.: Augochlora viridula, ('hloralieus sparsus se, versatus se, Curt isap is eoriacea, Evylarus 4-maenlatus, Halietus lerouxii, Oxystoglossa confusa, pura.

Other Hymenoptera (6)-Scelion.: Prosacantha n; Bracon.: Bracon trifolii, vernoniae, Dacnusa, Mierogastor ab, Opius mifeps.

Diptera (9)-Myeetophil.: Dynatosoma thoracicum n, lype; Syryh.: Chilosia petulea, Melanostoma obseurum, Rhingia; Empid.: Rhamphomyia piligeronis; Musc.: Morellia; Inthomy.: Hylrmyia plumosa, type, Mydaea socialis; Oscin.: Chlorops assimilis.

Coleoptera (3) - Inthic.: Corphyra terminalis; Curculion.: Inlostethus subealvus; Mordell.: Mordellistena biplagiata n.

Podophyllum peltatum $(9: 155-6)$.-Blonms April 14-May 21; 5 species and 8 individuals observed, A pril 25-May 14; Ma, W.

Long-tongued Bees (5) - Ap.: Apis e, 1; Bomb. (q) : Bombias separatus 1, Bombus americunorum 3, impatiens 1 ; Eucre.: Tetralonia dilecta $\sigma \vec{\sigma}, 2$.

\section{BigNONIACEAE}

Tecoma radicans.--Blooms June 8-Siptember 10 ; 3 visitors observed, July 21, August 6; $\mathrm{Ma}(0), \mathrm{R}$.

Birds (1)-Trochil.: Trochilus ab.

Long-tongued Bees (1)-Bomb.: Bombus amerieanorum 6.. $\mathrm{ab}, \mathrm{n}$.

Short-tongued Bees (1)-Halict.: Chloralictus aphyrus 9, e, n.

Extru-flural necturies.-Sixteen visitors observed, July 20, 21.

Ants $(4,8)$-Formir.: Formica fusea 13, schanfussi 3, Tapinoma 2; Myrmic.: Cremastogaster ab, day and night.

Short-fongued Bees (3)-Halict.: Chloralietus sparsns 9. 6. versatus $q, 20$, zephyrus $\delta^{7}+, 7$.

Diptera (9)--Syrph.: Syritta 1: Sarcophey.: Helieobia helieis 1, Sareophaga assilua 1, eimbieis 2 ; . I use.: Lueilia srieata 1, Musea 1, Stomoxys 1: Ayromyz.: Miliehiella 11; Ortal: Camptonura fy. 


\section{BoranginaCEAE}

Cynoglossum officinale (int).--One visitor observed, June 11; $\mathrm{Ma}, \mathrm{R}$.

Long-tongued Bees (1)-Megachil.: Megachile brevis q.

Lappula virginiana.-Blooms July 5-September 9, 4 visitors observed, July 15, 22, 27 ; Mi, W.

Short-tongued Bees $(3$, $)$-Halict.: Chloralictus sparsus, versatus, Seladonia.

Diptera (1)-Syrph.: Syritta fq.

Lithospermum canescens (8:144-6).-Blooms March 18-June 12 ; 37 visitors observed, April 18-June 5; Ma, Y.

Long-tongued Bees (17)-Ap.: Apis; Bomb. (q) : Bombias scutellaris, Bombus americanorum ab, impatiens; Ceratin.: Ceratina

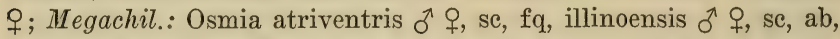
cordata $\delta$, distincta $q$, sc ; Nomad.: Gnathias ovatus $q$, Heminomada

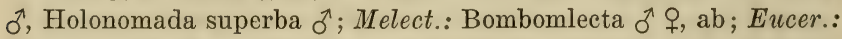
Tetralonia belfragei $\delta^{\lambda}$, dilecta $\delta \uparrow, a b$, in cop, illinoensis $\delta^{\lambda}$, type; Anthophor.: Anthophora $\delta$, fq.

Diptera (2)-Bombyli.: Bombylius atriceps, major fq.

Lepidoptera (18)-Nymphal.: Anosia fq, Charidryas, Phyeiodes, Vanessa atalanta, cardui, huntera; Lycaen.: Chrysophanus; Papilion.: Eurymus ab, Iphiclides fq, Papilio, Pieris; Hesperi.: Pholisora catullus, Thanaos brizo, icelus fq, juvenalis, martialis, persius; Noctu.: Plusia simplex.

Mertensia virginica (13:580-1).-Blooms March 31-May 25; 28 visitors observed, April 8-May 13; Ma, R.

Birds (1) -Trochil.: Trochilus.

Long-tongued Bees (16)-Ap.: Apis c; Bomb. (q): Bombias auricomus, separatus, Bombus americanorum fq, consimilis, ridingsii ; Megachil.: Centrosmia $\hat{\sigma}+$, sc, fq, Ceratosmia $\delta^{\lambda}+$, Osmia atriventris $\delta^{\lambda} q$, se, pumila + , se; Nomad.: Xanthidium dentariae $\sigma^{\tau}$; Eucer.: Tetralonia belfragei $\delta$, sc, fq, dilecta $\delta q$, fq, in cop, dubitata $\sigma^{\lambda}$, fq ; Anthophor.: Anthemoessa $\sigma^{\top}+$, Anthophora $\sigma^{\lambda} q, f q$.

Short-tongued Bees $(2,+\circ, \mathrm{n})-$ Halict.: Chloralictus versatus, Oxystoglossa confusa.

Diptera (3)—Syrph.: Rhingia sf, Teuchocnemis $\mathrm{f}, \mathrm{n}$; Bombyli.: Bombylius major.

Lepidoptera (6) - Nymphal.: Anosia, Vanessa atalanta; Papilion.: Euphoeades; Hesperi.: Thanaos juvenalis; Sphing.: Deilephila, Hemaris thysbe. 
Myosotis virginica.-Blooms April 27-June 9; 2 visitors observed, May 21, 24; Mli, W.

Short-tongued Bees (1)-Hulirt.: Oxystoglossa confusa f.

Lepidoptera (1)-Papilion.: Pontia.

\section{Campanutaceae}

Campanula americana $(4: 70)$.-Blooms July 1-Oetober $6 ; 20$ visitors observed, July 10-October 4 ; Ma, R.

Long-tongued Bers (9) - 1 p.: Apis; Bomb.: Bombias sejaratus $\hat{o}$, Bombus americanorun of $\delta$, fy, impatiens of 8 ; Wuguhil.: Megachilini: Megachile brevis of f. petulins o. e, Oligotropus eampamulae $\mathcal{d}$ o se, oligolegre, type; Coelioxyini : Coelioxys nodrota of; Eucer.: Melissodes bimaeulata $0^{\top}$ ㅇ․

Short-tongued Bees (7) - Halict.: Agrapostemon radiatus of ${ }^{\circ}$ Choralietus sparsus $q$, e, n, Curtisapis eoriacea $\hat{o}$ o. Halietus lerouxii of, Oxystoglossa confusa $q$. c. n ; Prosopid.: Prosupis ziziae ㅇ, $\mathrm{f}, \mathrm{n}$; Collet.: Colletes latitarsis $q, \mathrm{n}$.

Other Hymenoptera (1)-Scoli.: Elis 5-cineta.

Diptera (1) -Syrph.: Pipiza nigribarba f, $\mathrm{n}$.

Lepidoptera (2)-Nymphal.: Vanessa cardui; Hesperi.: Pholisora hayhurstii.

Specularia perfoliata.-Blooms May 18-June 29; 17 visitors observed, May 29-June 29; Mi, R.

Long-tongued Bees $(3, q)-$ Bomb.: Bombias separatus, Bombus americanorum; Ceratin.: Ceratina.

Short-tongued Bees (6)-Halict. (q): Augochlora fervida, Chloralietus versatus se, Oxystoglossa confusa, similis se. fy. Seladonia; Collet.: Colletes brevicornis oै ${ }^{+} . \mathrm{se}$, ab, oligolege.

Other Hymenoptera (1)-Eumen.: Leionotus foraminatus.

Diptera (4)—Syrph.: Mesogramma marginata $1, n$; Bombyli.: Anthrax sinuosa $\mathrm{f}, \mathrm{n}$; Comop.: Oneomyia, Stylogaster bianuulata.

Lepidoptera (3)-Lycaen.: Everes; Papilion.: Pieris ; Ifesperi.: Pholisora hayhurstii.

\section{Caprifoltaceae}

Lonicera sempervirens (eult, $10: 241$ ).- Мa (O), $\mathrm{R}$.

Birds (1)-Trochil.: Trochilus ab.

Lonicera sullivantii $(10: 241-4)$.- Blooms May 15-June $i ; .5$ visitors observed, May 17-27; Ma, Y.

Birds (1) -Trochil.: 'Trochilus fq.

Long-tongued Boes (3. f)-Bomb.: IBombus americanurum, impatiens fq; Anthophor.: Anthophora se, fq.

Diptera (1)—Syrph.: Pipiza femoralis $\mathbf{f}, \mathbf{n}$. 
Sambucus canadensis $(5: 274-5)$.-Blooms May 27-August 9; 32 visitors observed, June 7-July 11; Pol (Mis), W. All visits are for pollen.

Long-tongued Bees (2, c).-Ap.: Apis fq; Ceratin.: Ceratina $q$.

Short-tongued Bees $(6, q, \mathrm{c})-$ Halict.: Chloralictus sparsus ab, versatus ab, zephyrus ab, Evylaeus arcuatus, Halictus parallelus; Andren.: Trachandrena claytoniae.

Diptera (19, f) -Syrph.: Allograpta fq, Baccha fuscipennis, Eristalis dimidiatus, tenax, Mallota cimbiciformis fq, Mesogramma geminata, marginata, Orthoneura nitida ab, Spilomyia hamifera fq, Syritta, Syrphus americanus, ribesii fq, Xylota fraudulosa; Bombyli.: Anthrax sinuosa, Argyramoeba oedipus; Musc.: Musca, Pseudopyrellia; Anthomy.: Phorbia acra, platura.

Coleoptera (5, f)-Cerambyc.: Euderces; Dermest.: Attagenus fq; Malach.: Anthocomus; Mordell.: Pentaria; Scarabae.: Euphoria fulgida.

108 individuals taken on the flowers, July 8-10, 1920, showed:

Long-tongued Bees $(4, \mathrm{c})$-Apidae.

Short-tongued Bees $(68, q, \mathrm{c})$-Halictidae.

Diptera (32, f)-Syrphidae 31, Anthomyidae 1.

Coleoptera $(4, \mathbf{f})$.

Symphoricarpus orbiculatus $(16: 173-4)$.-Blooms July 6September 10 ; 120 visitors observed, July 8-August 30; Mis, Y.

Long-tongued Bees (10)-Ap.: Apis ab; Bomb.: Bombias separatus $q \Varangle$, Bombus americanorum $\Varangle$, se, consimilis $\not$, se, impatiens ㅇ s, $\Varangle$ sc, fq; Megachil.: Megachilini: Megachile mendica $\delta^{\pi} q$, Oligotropus $\delta^{\pi}$; Coelioxyini: Coelioxys 8-dentata $\delta^{\pi}$, sayi $\delta^{\pi} q$; Eucer.: Melissodes bimaculata ${ }^{\top}$.

Short-tongued Bees (27)-Halict.: Agapostemon radiatus ô 우 ab, Augochlora fervida $q$, viridula $\sigma^{\lambda} q, a b$, Chloralictus coreopsis $q$, cressonii $\delta^{\lambda} q, \mathrm{f} q$, obscurus $\delta^{\lambda} q, \mathrm{ab}$, sparsus $\delta^{\lambda} q, \mathrm{sc}, \mathrm{ab}$, tegularis $\delta^{1} q, \mathrm{fq}$, versatus $q, \mathrm{se}, \mathrm{ab}$, zephyrus $\delta^{\uparrow}+, \mathrm{ab}$, Curtisapis coriacea $\sigma^{\lambda} q$, forbesii $\delta$, Evylaeus foxii $q$, pectoralis $\delta$, fq, 4-macu-

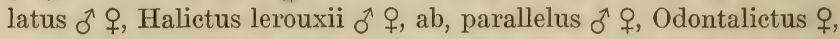

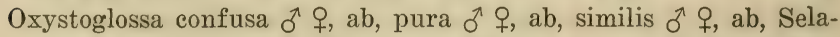
donia $\sigma^{\lambda} q$; Sphecodini: Sphecodes arvensis $\delta^{\lambda} q$, ab, heraclei $\delta^{\top} q$; Prosopid. ( $\sigma^{\top}+$ ) : Prosopis sayi, ab, ziziae fq; Collet.: Colletes nudus 0 \% $q$.

Other Hymenoptera (43)-Sphec.: Ammobia ichneumonea, nuda, pennsylvanica, Isodontia apicalis, macrocephala, Sphex gracilis, nigricans $\mathrm{fq}$, vulgaris $\mathrm{fq}$; Bembic.: Bicyrtes 4-fasciata, Megastizus; Scoli.: Elis obscura, 5-cincta fq, Scolia bicincta; Philanth.: 
Cerceris elypeata fig, eompacta, fumiponnis, Philanthus punetatus fq; Vesp.: Polistes anmularis, pallipes, variatus: Eumen.: Aucistro. cerus tigris, Eumenes fraternus ab, Leionotus anormis, arvensis. dorsalis, foraminatus ah, fulvipes, histromalis, illinoeusis fq. megatera, perennis, 4-sectus, serophuluriap, ziziae. Monobin, Zethus: Orybel.: Notoglossa frontalis; Larr.: Notogonidea, Tarliytes auru. lentus; Pompril.: Episyrou, Lophopompilus philadelphiens fip. Poecilopompilus navus, Prioenemoides mifasciatus.

Diptera (37)-Ciulic.: Ä̈les; Strationy.; Nemotelus. Stratio. myia meigenii: Syrph.: Eristalis tenax, transversus, Helophilus lartus, Mallota eimbieifurmis, Mesorramma reminata ab, marerinata, Milesia, Orthoneura nitida ah, Pipiza nigribarba, Spilomsia lonereornis, Syritta ah, Tropidia albistylum, quadrata; Emprid.: P'arempis ab; Bombyli.: Exoprosopa fasuipenuis: Comop.: Conops xamtho. pareus, Oneomyia: Twhin.: Arehytas analis fq, ateprima. Fulvoia bifaseiata, unifaseiata, Cyphoeera, Linnaemyia, Oeyptera dosiades, tenuis, Phoranthat magna, siphona, Syallanzania, Trichopoda pennipes. Trichophora, Xanthomelana; Musc.: Compsomyin, Lsucilia eaesar, Pseudopyrellia fq.

Lepidoptera (1)-Lycaen.: Cyaniris.

Coleoptera (2)-Mordell.: Mordella marginiata, Mord.llist-na pubeseens.

On suveu days, July 1:3-27, 1909, the following 87 species and 1,052 individuals were taken on the flowers:

Loug-tongued Bees $(6: 21)-1 p$.: Apis 12; Bomb.: Bombias separatus 1, Bomhus eonsimilis 1, impatiens 5; Mrgachil.: Oligotropus 1; Eucer.: Melissodes bimaculata 1.

Short-tongued Bees (25:781)-Maliet.: A gapostemon raliatus 26. Augochlora fervida 1, viridula 73, Chloralietus coreopsis 1. cressonii 7, obecurus 29. sparsus 200, tegularis 6, versatus 69. aephyrus 17. Curtisapis forbesii 2. Evylaeus foxii 1, pectoralis 9, 4-maculatus 3, Halietus lerouxii 15, paralellus 4, Oxystoglema enufusa 84. pura 22. similis 16:3, Seladonia 2: Spheeodini : Spheorles arvenais 2, heradei 1 ; Prosopid.: P'rosopis sayi 16 , riziae 6 ; Collet : Colletes nudus 2.

Other Hymenoptera $(30: 104) \rightarrow$ Spher.: Ammobia iehmeumonea 2, penusylvaniea 1. Isodontia apienlis 1. Sphex graeilis 2., nigrieans 4, vulgaris 4: Scoli.: Rlis olnamra 1, iecineta 3, Senlia hielaeta 3: Philanth.: Cereeris clypeata 5, compacta ‥ fumipennis 1. Philanthus punctatus 3; Verp.: Polistes pallipes 2. variatus 1; Rumen.: Ancistronerus tigris 1. Eumenes frateruus 10, Leionotus arvensis 3. foraminatus 31. fulvipes 3 , histrionalis 1. purennis 1. 4-ectus :2. serophulariae 1, Monubia 2, Yethus 2; Rarr.: Notogonidea 1, 
Tachytes aurulentus 1; Pompil.: Episyron 1, Lophopompilus philadelphicus 4.

Diptera (24:141)-Stratiomy.: Nemotelus 1; Syrph.: Eristalis tenax 1, transversus 1, Helophilus laetus 1, Mesogramma geminata 15, marginata 3, Milesia 1, Orthoneura nitida 20, Pipiza nigribarba 1, Spilomyia longicornis 1 , Syritta 10, Tropidia albistylum 1, quadrata 2; Empid.: Parempis 54; Conop.: Oncomyia 1; Tachin.: Archytas analis 6, Cyphocera 1, Siphona 1, Spallanzania 1, Trichopoda pennipes 4, Trichophora 2; Musc.: Compsomyia 4, Lucilia caesar 4, Pseudopyrellia 5.

Coleoptera (2:5)-Mordell.: Mordella marginata 4, Mordellistena pubescens 1 .

Triosteum perfoliatum (4:65).-Blooms May 3-June 17, 7 visitors observed, May 18, 23, June 3; Ma, R.

Long-tongued Bees (5)-Bomb. ( $($ ) : Bombus americanorum, consimilis ab, ridingsii; Anthophor.: Anthemoessa ô; Ceratin.: Ceratina $q, \mathrm{n}$.

Short-tongued Bees (2, $q, \mathrm{n})$-Halict.: Halictus lerouxii c, Oxystoglossa confusa se.

Viburnum prunifolium (10:237-8).-Blooms April 9-May 16; 83 visitors observed, April 24, May 8; Mis, W.

Long-tongued Bees (11)-Ap.: Apis sc; Ceratin.: Ceratina ô; Megachil.: Ceratosmia + , Osmia pumila + ; Nomad.: Gnathias cuneatus $\delta$, ovatus $\delta$, Heminomada $\delta$, Nomada cressonii $\delta$, fq, illinoensis $\delta$, sayi + , fq ; Eucer.: Tetralonia dilecta $\delta^{\lambda}$ ㅇ.

Short-tongued Bees (27)-Halict. ( $)$ : Agapostemon radiatus, Chloralictus eressonii, pilosus se, sparsus sc, ab, tegularis se, versatus se, ab, Evylaeus foxii se, pectoralis se, ab, Halictus lerouxii se, Oxystoglossa confusa se, fq; Sphecodini: Machaeris stygia, Proteraner, Sphecodes arvensis fq, Sphecodium cressonii; Collet.: Colletes inaequalis $\delta^{\top}$; Andren.: Andrena carlini $q$, dunningii $q$, erythrogastra $\delta^{\lambda}$, pruni $\delta^{\lambda} q, \mathrm{sc}, \mathrm{fq}$, in $\mathrm{cnp}$, sayi $\delta^{\lambda}+, \mathrm{sc}, \mathrm{fq}$, Opandrena cressonii $\delta^{\lambda} q$, se, fq, miserabilis $\delta^{\lambda} q$. se, ab, personata $\delta^{\lambda} q$, $\mathrm{ab}$, Trachandrena claytoniae $q$, sc, forbesii $q$, sc, hippotes $q$, sc, rugosa ${ }^{\pi}$.

Other Hymenoptera (2)-Vesp.: Polistes pallipes; Eumen.: Ancistrocerus tigris.

Diptera (36)-Stratiomy.: Stratiomyia quaternaria fq ; Syrph.: Brachyopa, Chilosia capillata fq, petulca, punctulata, Chrysogaster ontario, Criorhina decora, umbratilis, Eristalis aeneus fq, dimidiatus, transversus, Helophilus latifrons, Mesogramma geminata, marginata, Myiolepta strigilata $\mathrm{fq}$, Orthoneura pictipennis, Paragus 
bicolor, I'silota, Syritta ; Empid.: Empis nuda, otiosa fq, Rhamphomyia mutabilis, priapulus, sordida ; Bombyli.: Bombylius major, pulehellus; Conop.: Myopa vesieulosa fq, in eop; Tuchin.: Arehytas analis, Gonia capitata, Gymnosoma, Peleteria fq; Musc.: Graphomyia, Lueilia caesar, serieata fq, Phormia regina, Pseudopyrellia.

Lepidoptera (7)-Nymphal.: Charidryas, Vanessa huntera; Lycuen.: Cyaniris, Uranotes; IIesperi.: Thanaos juvenalis fị, martialis; Sphing.: Hemaris thysbe.

Viburnum pubescens $(10: 236-7 ; 16: 171-2)$.-13looms May 225; 71 visitors observed, May 4-20; Mis, W.

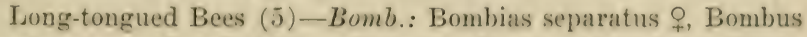
amerieanorum $q$; Ceratin.: Ceratina $q$, se; Megachil.: Osmia pumila $q$; Eucer.: Tetralonia dilecta 0 .

Short-tongued Bees (21)—Inlict. (q): Chloralictus eressonii se, versatus se, Curtisapis coriacea, se, Evylaeus foxii se, pectoralis sc, fq. 4-maculatus, Oxystoglossa confusa se; Prosopid.: Prosopis

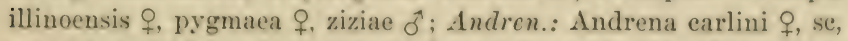
mandibularis $q$. se, fq, salietaria $q$, sayi $q$, se, fq, Opandrena eressonii $\sigma^{3}+$. se, $a b$, personata $\sigma^{*}, \mathrm{fq}$, serotina $q$, se, fq, Trachandrena elaytoniae $q$, se, ab, forbesii + , se, nuda $q$, se, rugosa $q$, se, fq.

Diptera (26)-Syrph.: Chilosia capillata, Criorhina deeora, umbratilis fq. Mesogramma geminata, Syritta, Volueella vesiculosa, Iylota chalybea; Empid.: Empis avida, compta, distans $\mathrm{fq}_{\mathrm{q}}$, humilis fq. levieula, nuda, otiosa, Pachymeria fq, Rhamphomyia angustipennis, exigua, mutabilis $\mathrm{f} q$, priapulus $\mathrm{fq}$, sordida ; Conop.: Myopa resieulosa ; Tachin.: Epigrimyia floridensis, Siphona; Muse.: Lucilia eaesar; Oscin.: Chlorops proxima, Iippelates plebejus.

Lepidoptera (2)-Papilion.: Iphiclides; IIesperi.: Thanaos juvenalis.

Coleoptera (17)-Bruch.: Bruchus hibisci; Cerambyc.: Callimoxys sanguinicollis, Euderees, Molorehus ab; Chrysomel.: Diabrotica vittata, Disonycha; Dermest.: Anthremus musaeorum iq, Cryptorhopalum triste, Orphilus ab ; Lampyr.: Telephorus dentiger; Mordcll.: Mordellistena aspersa $\mathrm{fq}$, biplagrata $\mathrm{fq}$, grammica; Oedemer.: Asclera ruficollis; Scarabae.: Euphoria fulgida, Hoplia $\mathrm{fq}$, Valgus gn, $\mathrm{n}$.

\section{Caryopuyluaceae}

Cerastium nutans.-Blooms April 16-May 31; 16 visitors observed, May 1-3; Mi, W.

Long-tongued Bees (1)-Ap.: $\Lambda$ pis fq.

Short-tongued Bees (4, \%)-Hulict: Chloralictus sparsus, versatus se, Halictus lerouxii, Seladonia se, fq. 
Diptera (9)-Syrph.: Eristalis bastardi, Helophilus latifrons fq, similis fq, Melanostoma mellinum, Mesogramma marginata $f$, Sphaerophoria; Bombyli.: Bombylius major; Tachin.: Peleteria; Musc.: Lucilia caesar.

Lepidoptera (2)-Nymphal.: Phyciodes; Papilion.: Eurymus.

Cerastium vulgatum (int).-Blooms May 6-September 24; 15 species and 28 individuals observed, May 19, 21, 28, June 5, 11; Mi, W.

Long-tongued Bees (4:9)-Ap.: Apis 5; Bomb.: Bombus americanorum,+ 1 ; Ceratin.: Ceratina,+ 2 ; Stelid.: Microstelis $q, 1$.

Short-tongued Bees $(4: 8, q)$-Halict.: Oxystoglossa similis 5, Seladonia 1; Prosopid.: Prosopis pygmaea 1; Andren.: Opandrena personata 1 .

Other Hymenoptera (1:1)-Eumen.: Leionotus illinoensis 1.

Diptera (6:10)—Syrph.: Mesogramma marginata 2, Sphaerophoria 4; Bonıbyli.: Toxophora 1; Conop.: Zodion fulvifrons 1; Tachin.: Cistogaster pallasii 1, Peleteria 1.

Saponaria officinalis (int).-Blooms June 1-October 7;2 visitors observed, August 24; Ma, R.

Lepidoptera (2)-Papilion.: Euphoeades, Pontia.

Silene nivea.-Blooms June 10-July 9; 1 visitor observed, June 6; Mi, W.

Short-tongued Bees (1)-Halict.: Chloralictus pilosus $q$.

Silene stellata.-Blooms June 18-August 7; 1 visitor observed, June 23 ; Ma, W.

Long-tongued Bees (1)—Bomb.: Bombus americanorum ఫ̧.

Stellaria longifolia.-Blooms May 7-June 26; 13 species and 31 individuals observed, May 14, 18, 22, 24; Mi, W.

Long-tongued Bees (3:3)-Megachil.: Diceratosmia \&, 1; Nomad.: Holonomada superba $\sigma^{\lambda}, 1 ;$ Eucer.: Tetralonia dilecta $\sigma^{\lambda}, 1$.

Short-tongued Bees $(6: 17)$ - Halict. (q): Chloralictus sparsus 3, versatus se, 1, Oxystoglossa similis 5, Seladonia 5; Prosopid.: Prosopis ziziae ô, 2; Andren.: Opandrena personata $q$, sc, 2.

Diptera (4:11)-Mycetophil.: Sciara atrata 2; Syrph.: Helophilus laetus 1, Mesogramma marginata 2; Tachin.: Cistogaster pallasii 6.

Stellaria media (int, 6:267-8).-Blooms March 16-December 1; 61 species observed, March 25-May 8 and October 15; Mi, W.

Long-tongued Bees (6)-Ap.: Apis; Ceratin.: Ceratina ${ }^{1}$; Megachil.: Ceratosmia $\delta^{\lambda}$; Nomad.: Gnathias ovatus $\delta^{\lambda}+$, Xanthid. ium luteoloides ơ, luteolum ơ. 
Short-tongued Bees (19)-IIulict.: Chloralietus obseurus of,

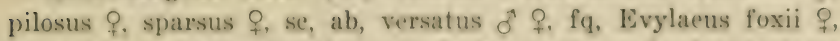
4-maculatus of. Halietus lerouxii of, Odontalietus $\mathcal{f}_{\text {, Oxystoglossa }}$ confusa + , ab, similis + . Seladonia $q$; Collet.: Colletes inaequalis of; Andren.: Andrena illinoensis $q$, sayi \&. Opandrena eressonii of ?, se, in cop, type, miserabilis of q. personata $d ?$ ? ab, Paraudrena $d$, Trachandrena forbesii $q$.

Other Hymenoptera (4)-Sapyg.: Sapyera: Chalcid.: Smicra torvina; Ichneumon.: Pimpla novita ; Tenthredin.: Dolerus arvensis.

Diptera (29)-Mycetophil.: Seiara atrata; Syrph.: Brachypalpus frontosus, Chilosia eapillata, Eristalis aeneus, dimidiatus, tenax, Melanostoma obscurum, Mesogramma geminata, marginata fq. Platyehirus quadratus, Syritta, Syrphus amerieanus, ribesii; Tachin.: Gonia capitata ab, exul fq. Siphona ; Sarcophag: ('ynomyia fq; Musc.: Lueilia caesar, sericata, sylvarum, Musca, Myospila, Phormia terraenovae, Pollenia, Pseudopyrellia ; Anthomy.: Phorlia acra, platura; Berbor.: Borborus; Scatophag.: Seatophaga.

Lepidoptera (2)-Nymphal.: Vanessa huntera; Lycaen.: Cyaniris.

Hemiptera (1)-Lygae.: Lygaeus.

May $6,8,1916$, the first 100 individuals, representing 19 species, taken on the flowers are as follows:

Long-tmgned Bees $(1: 2)$-Nomal.: Gnathias ovatus 2.

Short-tongued Bees (7:73)-Malict.: Chloralictus sparsus 40, versatus 5. Evylaeus 4-maculatus 2, Oxystoglosal similis 2; Andren.: Opandrena cressonii 2, miserabilis 1, personata 21.

Other Hymenoptera $(1: 1)$-Sapyg.: Sapyga 1.

Diptera $(10: 24)$-Syrph.: Mesogramma geminata 2, marginata 6. Syritta 1; Tackin.: Siphona 3; Muse.: Lneilia eaesar 1, serieata 1, sylvarum 4. Pollenia 1; Anthomy.: Phorbia acra 4, platura 1.

\section{Celastraceae}

Celastrus scandens.-Plooms May 21-Jume :3 6 species and 15 individuals observed, May 24; Mi (Mis), Y.

Long-tongued Bees (1:1)-Megachil.: Hoplitis ?. 1.

Short-tongued Bees (4:13) - II alict.: Aurochlora fervida ?. 1 .

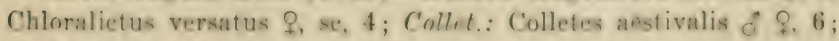
Andren.: Opandrena eressonii 9 , se, 2.

Other 11 ymenoptera $(1: 1)$ - Eumen. : Leionotus foraminatus 1 .

Evonymus atropurpureus $(16: 1.55)$ - Blooms May $25-J$ Jue 23 : 22 species and 51 individuals observed, Jume 7. 8. 11, 15; Mi, R.

Short-tougued Bees (5:11. 9)-Malint.: Chloralietus sparsus 5. versatus 3 , zephyrus 1 , Oxystoglossa confusa 1 , pura 1 . 
Diptera (15:34)-Syrph.: Allograpta 2, Baceha tarchetius 1, Mesogramma marginata 1, Syrphus ribesii 1; Sarcophag.: Boettcheria 4, Helicobia helicis 1, 4-setosa 4, Sarcophaga cimbicis 1, sinuata 1; Musc.: Myospila 4, Phormia regina 3, Pseudopyrellia 6; Anthomy.: Phorbia acra 3, platura 1; Ortal.: Seoptera 1.

Coleoptera (2:6)-Chrysomel.: Rhabdopterus 1; Mordell.: Mordellistena ornata 5 .

\section{Commelinaceae}

Tradescantia brevicaulis.-Blooms April 29-June 15; 13 visitors observed, May 8-24; Ma, R.

Long-tongued Bees $(6, \mathrm{c})-A p .:$ Apis $\mathrm{fq} ;$ Bomb.: Bombias separatus $q$, Bombus americanorum $q$; Ceratin.: Ceratina $q$; Megachil.: Megachile brevis $q$; Eucer.: Tetralonia dilecta + , fq.

Short-tongued Bees (7, $q, \mathrm{c})-$ Halict.: Chloralictus tegularis, versatus, Evylaeus pectoralis, 4-maculatus, Oxystoglossa similis, Seladonia; Andren.: Opandrena cressonii.

Tradescantia pilosa.-Blooms June 8-September 27; 3 visitors observed, July 1; Ma, R.

Long-tongued Bees $(2, \not{+}, \mathrm{c})-$ Bomb.: Bombus americanorum, impatiens.

Short-tongued Bees (1)-Halict.: Oxystoglossa pura + , c.

Tradescantia virginiana $(5: 71)$.-Blooms May 8-August 11 ; 18 visitors observed, June $3-17$; Ma, $\mathrm{R}$.

Long-tongued Bees (7, e)-Ap.: Apis; Bomb.: Bombias auricomus $q$, separatus $q \not$, Bombus americanorum $q$, consimilis $\Varangle$, se, impatiens $q$; Ceratin.: Ceratina $q$; Eucer.: Tetralonia dilecta $q$.

Short-tongued Bees (2, ㅇ, c)-Halict.: Agapostemon viridulus, Chloralictus pruinosus.

Diptera (8, f, n)-Syrph.: Allograpta, Eristalis dimidiatus, tenax, Mesogramma marginata, Sphaerophoria, Syrphus americanus, ribesii, Tropidia mamillata.

Coleoptera (1)-Curculion.: Centrinites $f, n$.

\section{Compositae}

Achillea millefolium.-Blooms May 18-August 10; 47 visitors observed, May 28-June 28; Mis, W.

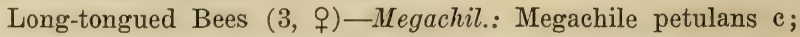
Bomb.: Bombias auricomus e; Nomad.: Gnathias euneatus.

Short-tongued Bees $(4, q)$-Halict.: Chloralictus pilosus se, versatus sc, ab, Odontalictus se, Paralictus simplex.

Other Hymenoptera (8)-Crabron.: Protothyreopus rufifemur; Oxybel.: Notoglossa americana, frontalis, Oxybelus packardii; 
Philunth.: Cererris compaeta, rufinoda ; Eumen.: Leionotus foraminatus, fulvipes.

Diptera (34) - Tuban.: Chrysopss striatus; Memestrin.: Mhynchocephalus; Bombyli.: Oneodoceria, 'Toxuplora; Syrph.: Allograpta, Eristalis aeneus, dimidiatus, tenax, transversus Mallota cimbieiformis, Mesogramma marginata, Orthoneura nitida, Paragus tibialis, Sphaerophoria, Syritta, Syrphus americanus, ribesii; Conop.: Conops brachyrrhynehus, Oncomyia; Tuchin.: Apinops, Arehytas analis, Cistogaster oecidua, pallasii, Epigrimyia polita, Gymnosoma, Senotainia rubriventris, trilineata; Sarcophag.: Helieobia helicis, Sareophaga eimbieis; Musc.: Lucilia sylvarum, Pseudopyrellia; Anthomy.: Phorbia acra, platura ; Sapromyz.: Sapromyza vulgaris.

Coleoptera (1)-Mordell.: Mordella melaena.

Actinomeris alternifolia.-Blooms August 15-Oetober 4, 12 visitors observed, August 17-September 12; Mas, Y.

Long-tongued Bees (6)-Bomb.: Bombias separatus $\delta$, Bombus

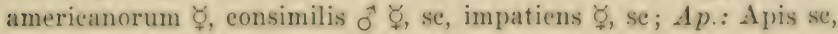
fq; Epeol.: Triepeolus remigatus + .

Short-tongued Bees (2)-Malict.: Curtisapis coriacea of ; Panurg.: Pseudopanurgus labrosiformis ${ }^{+}, \mathrm{c}$.

Other Hymenoptera (1) -Scoli.: Scolia bicineta.

Diptera (1)—Syrph.: Syrphus ribesii. ..

Lepidoptera (1)-Lycaen.: Cyaniris.

Coleoptera (1)-Lampyr.: Chauliognathus pennsylvanieus, in cop.

Ambrosia trifida (anemophilous).-Two species and 33 individuals observed, August 27, September 1.

Long-tongued Bees $(1: 31)-A p .:$ Apis e, 31 .

Other Hrmenoptera $(1: 2)$-Vesp.: Vespula germaniea, expl., 2.

Antennaria plantaginifolia $(15: 462-3)$ - - Blooms $\Lambda$ pril 4-May $21 ; 72$ visitors observed, April 14-May 5; Mis, W.

On staminate flowers (25):

Long-tongued Bees (3)-Megachil.: Osmia pumila do ; Bomb.: Bombus amerieanorum $f$; Nomad.: Nomada eressonii of.

Short-tongued Bees (2, f)-Mulict.: Evylaeus foxii se; Sphecodini : Dialonia antennariae type.

Diptera $(14)$-Empil.: Empis otiosa type; Syrph.: Eristalis aeneus, Mesogramma geminata, Orthoneura nitida, Paragus tibialis. Platyehirus hyperboreus, quadratus: Tochin.: Pachyophthalmus signatus; Sarcophag.: Cynomyia, Helicobia belicis, Sareophaga 
cimbicis; Musc.: Morellia; Anthomy.: Limnophora; Oscin.: Chlorops versicolor.

Lepidoptera (4)-Nymphal.: Vanessa huntera; Hesperi.: Thanaos juvenalis; Noctu.: Drasteria erechtea ; Pyraust.: Loxostege.

Coleoptera (2, f)-Dermest.: Anthrenus scrophulariae; Oedemer.: Asclera ruficollis.

On staminate and pistillate flowers (26):-

Long-tongued Bees (5)-Ceratin.: Ceratina $\delta$, ab, Zaodontomerus $\sigma^{\lambda}$; Ap.: Apis; Nomad. ( $\left.\sigma^{\lambda} \uparrow, \mathrm{fq}\right)$ : Gnathias ovatus, Nomada sayi.

Short-tongued Bees (12)-Halict. () : Chloralictus pilosus se, sparsus se, ab, versatus se, ab, zephyrus, Halictus lerouxii ab, Odontalictus sc, ab, Oxystoglossa confusa fq, similis se, fq, Seladonia se, ab; Sphecodini: Sphecodes minor; Andren.: Parandrena $q$, Trachandrena claytoniae $\delta$.

Diptera (7)—Empid.: Rhamphomyia priapulus; Syrph.: Mesogramma marginata in cop, fq, Sphaerophoria fq; Tachin.: Gonia capitata ab; Musc.: Lucilia caesar, Pseudopyrellia; Anthomy.: Phorbia platura.

Lepidoptera (2)-Lycaen.: Cyaniris; Papilion.: Eurymus.

On pistillate flowers (21) :-

Long-tongued Bees (3)-Nomad.: Heminomada $\sigma^{\star}$, Phor $\sigma^{\star}$, Xanthidium luteolum $q$.

Short-tongued Bees (11)-Halict. (q): Chloralictus pruinosus, Evylaeus arcuatus, pectoralis; Sphecodini: Sphecodium cressonii; Andren.: Andrena erythrogastra $\delta^{\lambda}$, illinoensis $\delta^{\lambda}+, \mathrm{fq}$, nigrae $q$, salictaria $\delta$, ab, sayi $q$, Opandrena miserabilis $\delta$, Trachandrena forbesii $\delta$.

Other Hymenoptera (1)-Chrysid.: Holochrysis hilaris.

Diptera (5)-Bombyli.: Bombylius major; Syrph.: Paragus bicolor; Tachin.: Euphorocera claripennis, Gonia exul; Anthomy.: Phorbia acra.

Lepidoptera (1)-Hesperi.: Thanaos brizo.

Anthemis cotula (int).-Blooms May 17-October 24; 7 visitors observed, June 21, 22 ; Mis, W.

Short-tongued Bees (5)-Halict.: Chloralictus sparsus $q$, sc; Halictus lerouxii $q$, Odontalictus $q$, se, fq, Oxystoglossa similis $q$, sc, Seladonia $\delta$ ․

Diptera (2)-Empid.: Parempis; Conop.: Oncomyia.

Arctium minus (int).-Blooms July 7-October 24; 19 species and 44 individuals observed, July 21-September 8; Mas, R. 
Long-tongued Beres $(9: 26)$-Megechit: Antheruois P, ac, 1 ,

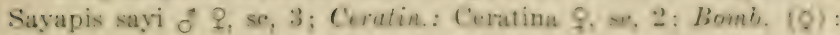
Bombus amerieanorum se, 4. consimilis 1 , impatiens on. 2; $A$ p.: $A$ pis 7; Eucer.: Melisodes bimaeulata of 2, 3, semonians of, 3.

Short-tongued Bees $(4: 11)$ - Ueliet: I Irapuetemon radiatun

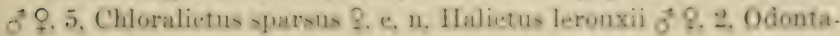
lietus ㅇ, 2.

Diptera (2:2)-Bonbyli.: Exoprosopa faseiata. 1; Syrph.: Eristalis tenax 1.

Lepidoptera $14: 5)$-Popilion. Eurymus 1. Pieris 2: IIspreri: Hesperia 1, Limochores taumas 1.

Aster anomalus.-Blooms August 2.-Xovember 8; 34 visiter observed, August 22-October 9; Mas, R.

1.me-tongued Bees (10)-Meguchil.: Megachile brevis o, oc. Xanthosarus f. se; Ceratin. Ceratina 9 , se: Bomb.: Bombus annicanorum of $\$$. fq. impatiens o $\$$. Psithyrus variabilis d; Ap. Apis se: Eucre: Melisodes autumnalis of 9 , se, simillima 9 , se, trinodis $q$, se.

Short-tongued Bess (4)-IIulict.: Chloralietus pilosus d. Ery-

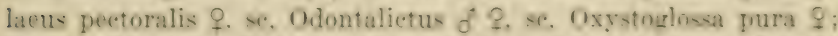
Collet. (q. se) : Colletes amerieanus. eorppactus; Andren. (Q. se): Pterandrena asteris solidaginis.

Other Hymenoptera (1)-Bracon.: Chelonus.

Diptera (8)-Bombyli: Anthrax haleyon, Geron rufipes. Sirarunpelius: Empril.: Parempis; Syrph.: Eristalis transversus, Syrphus areuatus; Tachin.: Siphoplagia anomala; Muss.: Componyia.

Lepidoptera (5)-Nymphal.: Phreiodes: Popilim.: Callidryss, Pieris, Pontia, Zerene.

Coleoptera (1)-Melo.: Epieauta pennsylvaniea, n.

Hemiptera (1) - Lygae: Lygaeus.

netuber $6,1909,2.2$ speris and 66 indiviluals sem taken $\mathrm{m}$ the flowers, as follows:

Long-tungued Bees $\langle 7: 4: 3\rangle$-Meguchil.: Megachile brevis 1: Xanthesarus 9: Bomb.: Bombus americanorum 10; impatiers 5: Ap.: Apis 8 ; Eurr.: Melissoules autumnalis 9; trinodis 1.

Short-tongrued Bees $(6: 12\}$-Halict.: Olontalietus 1: Oxystoglowa purs 1 ; Collet.: Colletes americanus 2: compactus 6; . A sirco.: Pterandrena asteris 1 ; solidaginis 1 .

Other Hymenoptera $(1: 1)$-Bracon.: Chelonus 1.

Diptera (4:5)-Bombyli.s Spurnopolius 2: Empid.: Parewpis 1; Syrph.: Syrphus arcuatus 1; Musc.: Compsomyia 1.

Lepidoptera 2:3)—Papilion.: Pieris 1; Pontia 2. 
Coleoptera (1:1)-Melo.: Epicauta pennsylvanica 1. Hemiptera (1:1)-Lygae.: Lygaeus 1.

Aster ericoides villosus (16: 175-6).-Blooms August 30-November 8; 302 visitors observed, August 30 -November 8; Mis, W.

Long-tongued Bees (37)—Stelid.: Stelidium o; Megachil.: Trypetini: Neotrypetes productus $\hat{\sigma}$, truncatus + , se; Megachilini : Megachile brevis $q$, se, generosa $q$, mendica $q$; Sayapis sayi $q$, se, Xanthosarus $\delta \hat{q}$, se; Coelioxyini: Coelioxys 8-dentata $\delta^{\lambda} q$, fq, rufitarsis $q$, sayi $q$; Ceratin.: Ceratina $\delta$ $q$, fq, Zaodontomerus $\delta^{\wedge}$,

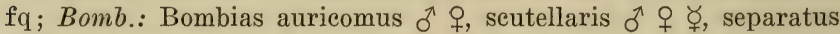

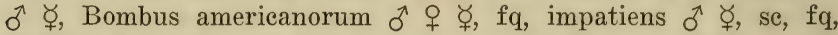
Psithyrus variabilis of, $\mathrm{fq} ; A p .:$ Apis se, $\mathrm{fq}$; Nomad.: Holonomada

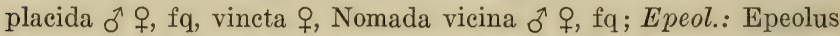
autumnalis $\delta$, bifasciatus $q$, pusillus $\delta$, $q$, fq, Triepeolus concolor $q$, cressonii $\delta^{\tau} q$, donatus $\sigma^{\top}+$, fq, micropygius $q$, type, pectoralis $o^{\lambda}$ q, fq, type; Eucer.: Epimelissodes obliqua $\sigma^{\lambda}$, Melissodes asteris

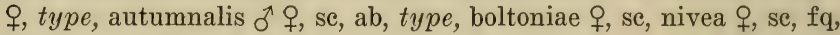
simillima $\sigma^{\tau}+$, se, fq.

Short-tongued Bees (53)-Halict.: Agapostemon radiatus $\delta$, fq, splendens $\sigma^{\lambda} q$, texanus $\sigma^{\lambda} q$, se, viridulus $\delta^{\lambda} q, \mathrm{se}, \mathrm{fq}$, Augochlora fervida $\delta^{\lambda} q$, se, viridula $\sigma^{\lambda} q$, Chloralictus coreopsis $\hat{\sigma} q$, foveolatus $\delta^{\lambda}$, type, illinoensis $\delta$, se, fq, obscurus $\delta$, fq, pilosus $\delta$, fq, pruinosus $\delta^{\lambda}$, smilacinae $\delta$, sparsus $\delta$, se, tegularis $\delta^{\lambda} q$, versatus $\delta^{\lambda}+, \mathrm{se}, \mathrm{ab}$, zephyrus $\delta^{\tau}+, \mathrm{fq}$, Curtisapis coriacea $\delta^{\lambda}$, forbesii $\delta^{\tau}+, \mathrm{se}$, fuscipennis $\delta$, fq, Dialictus $\delta \uparrow, f q$, Evylaeus arcuatus $q$, foxii $q$, pectoralis $\delta$, se, fq, 4-maculatus $q$, Halictus lerouxii $q$, se, parallelus $\delta$, Odontalictus $\delta$, se, ab, in cop, Oxystoglossa confusa $\delta^{\lambda}+, \mathrm{se}, \mathrm{ab}$, pura $\delta^{\lambda}+$, similis $\delta^{\lambda}+, \mathrm{fq}$, Paralictus platyparius $q$,

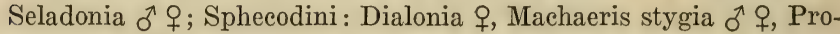
teraner $\hat{\sigma} q$, Sphecodium cressonii $\hat{\sigma}$ ㅇ, fq; Prosopid.: Prosopis illinoensis + , pygmaea $q$; Collet. $\left(\sigma^{\lambda}+, \mathrm{sc}\right)$ : Colletes americanus $\mathrm{fq}$, armatus, compactus, fq, eulophi ; Andren.: Andrena nubecula $\delta^{\pi}+$ se, $f q$, Pterandrena asteris $\sigma^{\top} q, \mathrm{se}, \mathrm{f} q$, in cop, pulchella $q$, solidaginis $\widehat{\delta}+$, se, fq; Panurg.: Calliopsis andreniformis $q$, se, Heterosarus $q$, se, Perdita $q$, se, Pseudopanurgus albitarsis $\delta^{\lambda}$, asteris $\sigma^{\lambda}+$, sc, ab, in cop., type, compositarum $\sigma^{\top}+$, se, ab, in cop.

Other Hymenoptera (54)-Crabron.: Hypocrabro, Protothyreopus rufifemur, Solenius; Philanth.: Cerceris astarte, bicornuta, clypeata, fulvipes, Philanthus punctatus, ventilabris; Pemphredon.: Mimesa; Sphec.: Ammobia ichneumonea, Priononyx atrata, fq, Sphex gracilis in cop, nigricans, pictipennis, procera, vulgaris; Larr.: Larropsis, Notogonidea, Tachytes obscurus; Bembic.: Bi- 
cyrtes ventralis, Stizus; Pompil.: Anoplins illinomsis, Ceropales iraterna, Lophoponpilus philudelphieus, Planiens feralis: Vesp.: Polistes annularis, pallipes, variatus, Vespula euneata. germaniea i1]. maeulata; Eumen.: Aneistroeerus capra, campetris, tigris fq, Eumenes fraterms, lecionotus anomis iq, arvensis, elyjostus, dorsalis, foraminatus, fulvipes, fundatus, histrionalis, Zendalia zendaloides; scoli, : Elis interrupta, 5-cineta, Scolia bicineta; Chrysid.: Gonochrysis: Chalcid.: Chaleis; Ichneumon. Lxetastes obseurus, Metopius polyeinctorius; Bmeon.: Agathis vulgaris, Chelinus.

Diptera (119)-Tipul.: Cieranomyia; Bibion: Dilophus serotinus; Bombyli.: Anthrux alternata fo, hypomelas, Areyramoeba albofasciata f, n, Exoprosoya decora, fasciata, Luseignenuis, Geron rufipes fq, Sparnopolius fq. Systropus fig, Toxophora; Empid.: Parempis; Syrph.: Allograpta iç. Baceha clavata fọ, Chilosia punctulata, Eristalis aeneus, bastardi, dimidiatus fq, flavipes, latifrons, tenax fq, transversus, Helophilus latifrons, similis fq, in eip, Mesogramma geminata, marginata ab, Milesia, Orthoneura nitida, Paragus bicolor, tibialis, Pipiza nigribara, pulchella, Platychirus quadratus, Sphaerophoria $\mathrm{f} q$, in eop. Spilomyia longieornis $\mathrm{fq}, 4$-faseiata, Syritta fq, Syrphus amerieanus fq. ribesii fq, Tropidia albistylum, mamillata, Xanthogramma emarginata; Cunop.: Conops xanthopareus, Oncomyia iq. Physocephala texana, tibialis, Robertsonomyia in cop. Stylogaster biannulata, Zodion fulvifrons $\mathrm{f} q$, nanellum fq; Tachin.: Actia, Alophora aeneoventris fq. pulverea, Apinops, Arehytas analis iq. aterrima. Belvosia bifasciata, unifasciata, Celatoria. Chaetogaedia crebra, Cistogaster oceidua fq, Clytiomyia, Distichona varia fq. Epigrimyia floridensis, Euphoweers elaripennis, Euthera, Gymnosima, Hypostena harbata. Leucustoma atra. neomexieana, nigrieornis, Linnaemyia fị, Macquartia, Ennyomma globosa, Oeyptera lata, tenuis, Paradidyma, Peleteria \&f. Phorantha asteris. magna, pruinosa, purpuraseens, Senotainia rubriventris, trilineata, Siphona fq. Siphoplagia anomala, flaecidirostris, Spallanzania fo. Sturmia phyeiodis. Trichopoda pennipes, Trichophom, Winthemia, Xanthomelana; Deri.: Ptiledexia; Sarcophay: Oynomyia, Helieohia helieis, 4-setosa, Sareophaga usoidua, dimbieis. hunteri; Musc.: Compsomyia íg. Graphomyia, Laeilia caestr, sericata fiq. Musca, Myospila, Preadopyrellin foq, Stomoxys fig: Anthomy.: Calythea, Coenosia anteunalis, antiea, 6-notata, Hylaemyia laevis, Limnophora. Phorbia acra fị. platura: Ortal.: Chastopuis: Trypet.: Trypeta humilis.

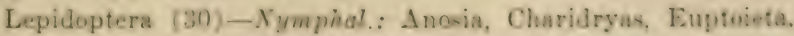
Iypatus, Junonia, Phyciodes fy, Vanes a candui, Auntera : Lyce n. : 
Chrysophanus, Everes, Uranotes; Papilion.: Callidryas, Eurema, Eurymus fq, Nathalis, Pieris, Pontia fq, Xanthidia, Zerene; Hesperi.: Atalopedes fq, Epargyreus, Hesperia fq, Limochores taumas, Polites; Ctenuch.: Scepsis fq; Noctu.: Drasteria erechtea, erichto, Feltia, Heliothis armiger, Plusia simplex.

Coleoptera (6)-Cerambyc.: Cyllene robiniae f, n; Chrysomel. $(f, n)$ : Diabrotica 12-punctata, longicornis, vittata; Lampyr.: Chauliognathus pennsylvanicus fq; Melo.: Epicauta pennsylvanica $f, n$.

Hemiptera (3)-Caps.: Lygus; Lygae.: Lygaeus fq; Beryt.: Harmostes.

September 28-November 6, 1909, the following 125 species and 1,129 individuals were taken on the flowers:

Long-tongued Bees (16:168)-Megachil.: Megachile brevis 2, Sayapis sayi 1, Xanthosarus 2; Ceratin.: Ceratina 10; Bomb.: Bombias auricomus 1, scutellaris 1, separatus 2, Bombus americanorum 33, impatiens 32 , Psithyrus variabilis $12 ;$ Ap.: Apis 52; Epeol.: Triepeolus donatus 1; Eucer.: Melissodes autumnalis 9, boltoniae 3 , nivea 3 , simillima 4 .

Short-tongued Bees (25:385)-Halict.: Agapostemon radiatus 3 , texanus 1 , Chloralictus coreopsis 3 , illinoensis 15 , obscurus 6 , pilosus 3 , sparsus 65 , tegularis 1 , versatus 166 , zephyrus 5 , Curtisapis fuscipennis 12, Evylaeus pectoralis 5, Odontalictus 18, Oxystoglossa confusa 13 , pura 4 , similis 13 , Paralictus platyparius 8; Sphecodini: Machaeris stygia 3; Collet.: Colletes americanus 18, compactus 1, eulophi 1 ; Andren.: Andrena nubecula 1, Pterandrena pulchella 1, solidaginis 4; Panurg.: Pseudopanurgus compositarum 15.

Other Hymenoptera (13: 29) - Philanth.: Philanthus punctatus 1; Sphec.: Ammobia ichneumonea 1; Vesp.: Polistes annularis 5; pallipes 3, variatus 4, Vespula germanica 1, maculata 5; Eumen.: Ancistrocerus capra 2, tigris 1, Leionotus anormis 2, arvensis 1, clypeatus 2 , foraminatus 1 .

Diptera (53:382)-Bibion.: Dilophus serotinus 4; Bombyli.: Sparnopolius 33; Syrph.: Allograpta 3, Eristalis latifrons 1, tenax 3, transversus 1, Helophilus similis 2, Mesogramma geminata 3, marginata 74, Sphaerophoria 14, Spilomyia longicornis 4, 4-fasciata 2, Syritta 10, Syrphus americanus 3; Conop.: Oncomyia 3, Zodion fulvifrons 1, nanellum 3; Tachin.: Alophora aeneoventris 2, pulverea 1, Archytas analis 10, aterrima 2, Belvosia unifasciata 1, Celatoria 1, Chaetogaedia crebra 1, Cistogaster occidua 7, Distichona varia 5, Euphorocera claripennis 2, Gymnosoma 3, Leucostoma atra 
1, Limnamyia 6. Paradidyma 4. Peleteria 2. Phorantha asteris 1. purpurascens 2. Senotainia rubriventris 1. Siphoplagia anomala 1. Spallanzania 5, Sturmia phyeiodis 2, Winthemia 1; Sarcophag.: Cynomyia 3. Itelieobia helieis 4, 4-setosa 2, Sareophaga assidua 3. cimbicis 6; Muse. Compsomyia 6. Lucilia earsar 8, serieata 23. Musea 4, Myospila 1. Pseudopyrellia 73. Stomoxys 6; Anthomy.: Phorbia acra 12 , platura 6.

Lepidoptera $(13: 88)$-Nymphal.: Phyciodes 15, Vanessa eardui 1, huntera 2; Lycuen.: Everes 1; Pupilion.: Eurymas 31, Pontia 1; Hesperi.: Atalopedes 2..2. Hesperia 3, Limochores taumas 1, Polites 3; Ctemuch.: Seepsis 6; Noctu.: Drasteria erechtea 1, Plusia simplex 1.

Coleoptera (5:75)-Chrysomel.: Diabrotiea 12-punetata 34. longieornis 5, vittata 3 ; Lampyr.: Chauliognathus pennsylyanieus 18; Melo.: Epicauta pennsylvanica 17.

Aster lateriflorus.-Blooms September 17 -Oetuber $30 ; 57$ visitors observed, September 17-Oetober 15 ; Mis, W.

Long-tongued Bees (9)--Megachil.: Megachile mendica f. se, Xanthosarus o. se; Cerulin.: Ceratina f. se, Zaodontomerus of; Bomb. (o f) : Bombus amerieanorum se, consimilis, impatiens se. ab; $A$ p.: Apis se, fy: Eurer.: Melissodes simillima $q$. se.

Short-tongued Bees (15)-Malict.: Agapostemon viridulus $q$. se, Augochlora viriclula $\delta$ f $q$, Chloralietus versatus $\delta$, Curtisapis coriacea $\delta$ f fuscipemis $\delta$, Oxystorlossa confusa $q$, pura $q$, similis १. Paralietus platyparius ôे; Collet.: Colletes americanus $q$. se.

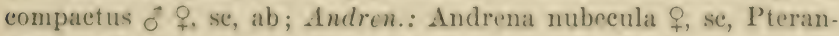
drena solidaginis $\&$; Panurg.: Pseudopanurgus asteris $f$, se, eompositarum of $\delta^{x}$, se.

Other Hymenoptera (14)-Crabon.: Svnothyreopus; Sphec: Sphex procera; Vesp.: Polistes annularis, ab, pallipes, fq, Vespula germanica, maculata; Eumen.: Aneistrocerus eapra, campestris, tigris, Eumenes fraternus, Leionotus anormis, foraminatus, strophulariae, Symmorphus debilis.

Diptera (17) - Syrph.: Baceha Pusripennis, Eristalis transversus, Helophilus simili, Suricomyia, Spilomyia longicornis, 4-fasciata, Syrphus americamus, torvus. Xanthogramma emarginata; Tachin.: Areliytas analis, aterrima, fig, Cistogaster oceidua, Cypho. cera, Leskiomima prina, 'Trichophora; Muse: Lueilia caesar, ah. Pseudopyrellia.

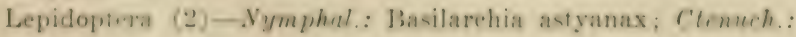
Scepsis.

September 2 - October 11, the following 37 species and $172 \mathrm{in}$. dividuals were taken as they came to the flowers: 
Long-tongued Bees (6:25)-Megachil.: Megachile mendica 2, Xanthosarus 2; Ceratin.: Ceratina 3; Bomb.: Bombus americanorum 3 , consimilis 1 , impatiens 14 .

Short-tongued Bees (11:37) — Halict.: Agapostemon viridulus 1, Augochlora viridula 5, Chloralictus versatus 1, Curtisapis coriacea 1 , fuscipennis 1 , Oxystoglossa confusa 2 , pura 1 , similis 5 ; Collet.: Colletes americanus 1, compactus 18; Panurg.: Pseudopanurgus asteris 1 .

Other Hymenoptera (8:50)-Sphec.: Sphex procera 1; Vesp.: Polistes annularis 18, pallipes 10, Vespula maculata 2; Eumen.: Ancistrocerus capra 7, tigris 4, Eumenes fraternus 6, Leionotus foraminatus 2 .

Diptera (10:57)-Syrph.: Eristalis transversus 1, Helophilus similis 3, Syrphus torvus 1; Tachin.: Archytas analis 1, aterrima 10, Cistogaster occidua 1, Cyphocera 1, Trichophora 1; Musc.: Lucilia caesar 36, Pseudopyrellia 2.

Lepidoptera (2:3)-Nymphal.: Basilarchia astyanax 1; Ctenuch.: Scepsis 2.

Aster multiflorus.-Blooms September 9-October 22; 43 species and 124 individuals taken on October $6,15,17$; Mis, W.

Long-tongued Bees (5:33)-Ap.: Apis 4; Bomb.: Bombus americanorum $\delta^{\lambda} \underset{+}{\phi}, 6$, impatiens $\delta^{\lambda}+, 17$, Psithyrus variabilis $\delta^{\lambda}, 2$; Eucer.: Melissodes simillima + , sc, 4.

Short-tongued Bees (8:44)-Halict.: Chloralictus illinoensis $q$, se, 1 , obseurus $q$, sc, 1 , pilosus $\uparrow, 1$, versatus $\sigma^{\top} \uparrow$, se, 33, Paralictus platyparius $q, 1$; Collet.: Colletes americanus $\hat{\jmath} \uparrow$, se, 5 , compactus ๆ, 1; Panurg.: Pseudopanurgus compositarum + , se, 1.

Other Hymenoptera $(11: 17) \longrightarrow$ Sphec.: Sphex gracilis 1, procera 1; Vesp.: Polistes annularis 3, variatus 4, Vespula maculata 1; Eumen.: Ancistrocerus catskillensis 2, Leionotus anormis 1, clypeatus 1; Crabron.: Solenius 1; Pompil.: Anoplius illinoensis 1, Lophopompilus philadelphicus 1 .

Diptera (13:21)-Bombyli.: Sparnopolius 2; Syrph.: Eristalis tenax 2, Mesogramma geminata 1, marginata 3, Paragus tibialis 2, Sphaerophoria 4, Syritta 1, Syrphus americanus 1; Tachin.: Archytas aterrima 1, Spallanzania 1; Sarcophag.: Helicobia helicis 1; Anthomy.: Coenosia antica 1, Limnophora 1.

Lepidoptera (2:2)-Papilion.: Nathalis 1, Pieris 1.

Coleoptera (1:4)—Chrysomel.: Diabrotica 12-punctata 4.

Hemiptera (3:3)-Core.: Alydus eurinus 1, pilosulus 1; Reduvi.: Sinea 1. 
Aster novaeangliae (15:459).-Blnous July 24-November 4 ; 41 visitors observed, July $2 \pm$-October 10 ; Mas, R.

Long-tongued Bees (13)-Megachil.: Merachile brevis f. se. fq. Xanthosarus do 9. se, fq; Corulin.: Ceratina 9. se; Romb.: Bumbias seutellaris $\delta$, separatus $\delta$, Bombus amerieanorum of $f \circ$. se. fq. impatiens of: Ap.: Apis se: Eucer. $(q)$ : Melisodes agilis se. autumnalis se, boltoniae se, nivea, simillima se.

Short-tongued Bees (7)-Halict.: Chloralietus enrespis 7 . e, n, Halictus parallelus $\hat{o}$, Odontalictus of; Collet.: Culletes americanus ‥ e, n: Andren.: Pterandrena helianthi P. e. solidaginis P. e. n; Panurg.: Pseudopanurgus compositarum $q, \mathrm{c}, \mathrm{n}$.

Diptera (11)-Bombyli.: Exoprosopa fascipennis, Sparnopolius. Systoechus; Syrph.: Eristalis dimidiatus, latifrons, tenax sf, transversus, Helophilus similis, Syrphus amerieanus f, n. Tropidia mamillata $\mathrm{f}, \mathrm{n}$; Conop.: Stylogaster biannulata fq.

Lepidoptera (10)-Nymphal.: Anosia, Phyciodes, Vanesa cardui; Papilion.: Eurymus fq, Pontia, Zerene; Hesperi: Hylephila, Polites; Noctu.: Ilelinthis armiger. Plusia simplex.

Aster paniculatus $(15: 460)$.-Blooms September 20-Oetober 17: Mis, W. The following 101 speeies and 229 individuals were taken as they came.

Long-tongued Bees (10:25)-Ap.: Apis 10; Bomb.: Bombus americanorum $\not$, . 1. impatiens $\delta$. 6; Ceratin.: Ceratina o. 1 ; Meguchil.: Trypetini: Neotrypetes trumeatus 9. 1; Megachilini: Megachile brevis of 9 , se, 2 . Xanthosarus 9,1 ; Coelioxyini : Coelioxys S-dentata . . 1; Eucer.: Melissodes boltoniae $q$. sc. 1. similima $q$. se, 1.

Short-tongued Bees (20):62)-Malict.: Agapostemon radiatus of,

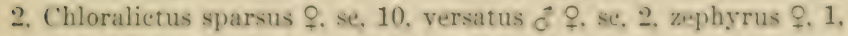
Curtisapis eoriacea of, 4, forbesii d, 1. Evylacus peetoralis ?. 1. Halietus lerouxii $q .1$, Odontalictus $f$. se, 22, Oxystoglossa enn-

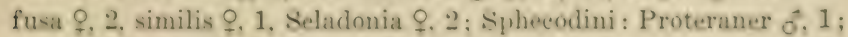
Prosopid.: Prosopis pyrmaca $\%, 1$; Collet.: Colletes americamus $q .1$. eompactus f, se, 2; Andren.: Andrena nubeeula f. 2, Pterandrena asteris $d$ P. se. 3. type, solidaginis 9. se. 1: Penurg.: Psoudopamurgus compositarum 오, se, 1.

Other Hymenoptera $(27: 51)$ - Philanth.: Cereeris prominens 1. Eucerceris 1. I'hilanthus politus 1, munetatus 1 ; Sphec.: Isolontia apicalis 2. Sphex gracilis 1 , nigricans 1 . procera 3 , vulearis 2 ; Larr.: Notogonidea 1: Pompil.: Episyron 1: Verp.: Polistes pallipes 3, rubiginosus 13, variatus 2. Viespula germunica 1. maculata 2: Eumen.: Ancistrocerus capra 1. eatulilleusis 1. tieris 1. Eumeues 
fraternus 2, Leionotus anormis 2 , arvensis 1 , foraminatus 2, scrophulariae 1; Scoli.: Elis 5-cincta 1; Ichneumon.: Lampronota varia 1; Bracon.: Bracon longicauda 1.

Diptera (28:67)-Syrph.: Eristalis aeneus 1, dimidiatus 1, tenax 4, transversus 4, Mesogramma geminata sf, 1, marginata 2, Sphaerophoria 1, Spilomyia 4-fasciata 1, Syritta 10, Syrphus americanus 1, ribesii f, 4; Conop.: Oncomyia 1; Tachin.: Alophora aeneoventris 1, Arehytas aterrima 3, Linnaemyia 1, Senotainia trilineata 1, Spallanzania 1, Trichopoda pennipes 1; Dexi: Ptilodexia 1; Sarcophag.: Sarcophaga cimbicis 1; Musc.: Compsomyia 5, Graphomyia 3, Lucilia caesar 1, sericata 6, Phormia regina 1, Pseudopyrellia 7, Stomoxys 2; Ortal.: Chaetopsis (s?) 1.

Lepidoptera $(12: 12)$-Nymphal.: Phyciodes 1, Vanessa cardui 1, huntera 1 ; Lycaen.: Cyaniris 1 ; Papilion.: Eurymus 1, Pieris 1, Pontia 1; Hesperi.: Atalopedes 1, Polites 1, Thanaos martialis 1; Ctenuch.: Scepsis 1; Noctu.: Plusia simplex 1.

Coleoptera (3:12)-Cerambyc.: Cyllene robiniae f, 1, $\mathrm{n}$; Lampyr.: Chauliognathus pennsylvanicus 10 ; Scarabae.: Euphoria sepulchralis $\mathrm{f}, 1, \mathrm{n}$.

Hemiptera (1:1)-Core.: Alydus eurinus 1.

Aster sagittifolius.-Blooms August $12-$ November $4 ; 40$ visitors observed, August 27-October 26; Mas, R.

Long-tongued Bees (11)-Ap.: Apis fq; Bomb. (ठ‡) : Bombus americanorum ab, consimilis, impatiens se, ab; Megachil. (q) : Megachile brevis se, mendica, Xanthosarus se; Epeol.: Epeolus pusillus ơ Eucer. (q) : Melissodes autumnalis se, nivea se, simillima.

Short-tongued Bees (8)-Halict.: Augochlora viridula + , se,

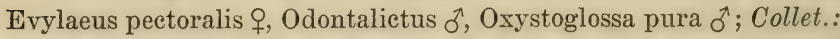
Colletes americanus $q$, sc, compactus + , se, ab ; Andren.: Pterandrena asteris + ; Panurg.: Pseudopanurgus asteris $\widehat{\sigma}+$, se, in cop.

Other Hymenoptera (3)—Sphec.: Sphex procera; Vesp.: Vespula germanica, maculata.

Diptera (11)—Syrph.: Mesogramma geminata, marginata, Spilomyia longicornis, 4-fasciata, Syrphus ribesii; Empid.: Parempis; Bombyli: Exoprosopa fascipennis; Sarcophag.: Cynomyia; Musc.: Compsomyia, Lucilia caesar, sericata.

Lepidoptera (3)-Papilion.: Callidryas; Hesperi.: Hesperia; Ctenuch.: Scepsis.

Coleoptera (4)-Chrysomel.: Diabrotica longicornis, vittata; Lampyr.: Chauliognathus pennsylvanicus; Melo.: Epicauta pennsylvanica $f, n$.

October 8, 21, 1909, the following 29 species and 81 individuals were taken as they came to the flowers: 
Loug-tongued Bees $(6: 33)-1 /$. Apis 3 ; Bumb. : liombus amerieanorum 10, impatiens 12; Megachil.: Xanthosarus 1 ; Eurr.: Melissodes autumnalis 6 , simillima 1 .

Short-tongued Bees (7:20)-Muliet.: Evylaeus puturalis 1. Odontalietus 3. Oxystoglossa pura 1: Collel.: Colletes americamus $\ddot{-}$ eompactus 11 ; Andren.: P'eramlrema asteris 1; P'anury.: I'sedulo. panurgus asteris 1.

Other Hymenoptera (3: 5)-Splice: Sples procera 1; Vesp.: Vespula germaniea 2, maculata 2.

Diptera $(7: 11)$ - Syrph.: Mesogramma geminata 1, marginata 1. Spilomyia longieornis 2. Syrphus ribusii 3; Empirl.: l'arempis 1: Musc.: Lucilia caesar 2, serieata 1.

Lepidoptera (2:4)- Hesperi.: Hesperia 1; ('tenurh.: Serpsis :3.

Coleoptera (4:8)-Chrysomel.: 1)iabroticat longieornis :3, vit. tata 1; Lamplr.: ('hauliognathus pennsylvanieus 2); Melo.: Epieauta pennsylvanica 2.

Aster salicifolius.-13looms September 2:3-0ctober :30; 44 species and 1:21 indiviluals taken on the flowers, Oefober 10-22; Mas, IR.

Loug-tongued Bees (10: 56) - Ap.: Apis 5; Bomb.: Bombias

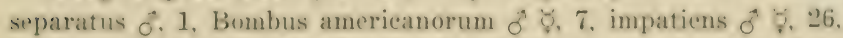
Psithyrus variabilis of, 10; Comlin.: Ceratina f. 1; Megachil.: Megarhile brevis $q, 1$. Xanthosarus $q$, se, 2); Eutr.: Melissodes agilis $q, 1$, simillima $q$, se, 2.

Short-toneued Bees ( $7: 13)$--Halict.: Agapostemon radiatus c Q. se, 2, Augochlora fervida $\delta, 1$, Chloralietus versatus $d, 2$, Odontalietus of 9 , se, 2 ; Andren.: Pterandrena asteris ${ }_{+}$, se, 2, solidaginis 9. se, 2; Panurg.: Pseudopanurgus compositarum $\&, 1$.

Other Hymenoptera (2:4)_Sphec.: Sphex proeera 3: Eumen: Leionotus arveusis 1.

Diptera $(16: 26)$ - syrph.: Eristalis tenax 1. 2. Helophilus similis 1 , Syritta 1, Syrphus amerieanus f, 1, ribesii $\stackrel{2}{-}$ Pomingli.: Sparuopolins 2; Conop.: Physocephala texana 1, Stylogaster biannulata 3: Tuchin.: Alophora auneoventris 1. Arehytas aualis 3. Spallanzania 1: Musc.: Compsomyia 3, Graphomyia 1. Lueilia earear 1, sylvarum 1, Pseudopyrellia 2.

Iepidoptrra $(8: 20)-N y m p h a l$ : Anoxia 1. Euptoieta 1, Vanena cardui 1. huntera 1; P'apilion.: Eurgums 12; Hesperi.: Atalopedos 2, Mesperia 1, Iylephila 1.

Coleoplera $(1: 2)$-Chrysumel.: Diabrotiea 12-pundata 2.

Aster turbinellus.- Bloms August 21-November 1; 26 spee ims

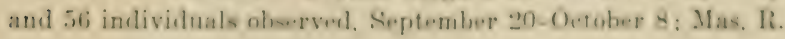


Long-tongued Bees (11:32)-Ap.: Apis se, $6 ;$ Bomb.: Bombias

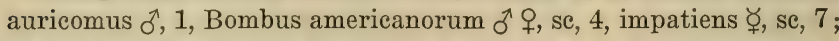
Megachil.: Megachilini: Megachile brevis $q$, se, 1, Xanthosarus $q$, c, 1; Coelioxyini : Coelioxys sayi ô, 1 ; Nomad.: Holonomada placida $q$, 1; Eucer. (q) : Melissodes autumnalis se, 8, nivea se, 1, simillima 1.

Short-tongued Bees (3:3)-Halict.: Agapostemon viridulus $0 \hat{\text {, }}$ 1, Curtisapis fuscipennis ô, 1; Collet.: Colletes compactus + , se, 1.

Diptera (8:13)—Syrph.: Helophilus similis 1, Mesogramma marginata 1, Pterallastes f, 1, Syritta 2, Syrphus arcuatus 1; Bombyli.: Anthrax halcyon 1, Sparnopolius 2, Systropus 3.

Lepidoptera (2: 6)-Nymphal.: Phyciodes 3 ; Hesperi.: Atalopedes 3.

Coleoptera (2: 3)-Chrysomel.: Diabrotica 12-punctata 2; Lampyr.: Chauliognathus pennsylvanicus 1.

Bidens aristosa (15: 473-4).-Blooms August 6-October 2; 133 visitors observed August 26-October 2; Mas, Y.

Long-tongued Bees (37)-Ap.: Apis ab; Bomb.: Bombias auricomus $\delta$, scutellaris $\widehat{\delta} \not{q}$, separatus $\widehat{\delta} \not{q}$, fq, Bombus americanorum $\delta^{\lambda} \not$, se, ab, impatiens $\delta^{\lambda}$ 우 $\not$, se, ab, Psithyrus variabilis $q$; Ceratin.: Ceratina + , se; Megachil.: Trypetini: Neotrypetes productus $\delta$, truncatus $\delta q$, se; Megachilini: Megachile brevis $\delta$, se, $f q$, mendica $q$, se, petulans $\delta \uparrow$, se ; Coelioxyini: Coelioxys 8-dentata $q$, sayi $\delta^{\lambda}$; Nomad.: Holonomada vineta $\delta^{\lambda}$, Nomada vicina $\delta^{\top}$; Epeol.: Epeolus autumnalis $\sigma^{\lambda}$, bifasciatus $q$, coreopsis $\sigma^{\lambda}$, type, pusillus $\delta^{\lambda}+$, $\mathrm{fq}$, Triepeolus concavus $\delta^{\lambda}$, concolor $\delta^{\lambda}+$, cressonii $\delta^{\lambda} q, f q$, donatus $\sigma^{\top} q$, lunatus $q$, pectoralis $\sigma^{\lambda}$, remigatus $\sigma^{\lambda}$; Eucer.: Epimelissodes atripes $q$, obliqua $\delta^{\lambda} q$, se, Melissodes agilis $\delta \uparrow, f q$, autumnalis $\widehat{\delta^{\lambda}}+$, se, bimaculata $q$, boltoniae $q$, se, ab, nivea $\hat{\delta}$, simillima $\widehat{\delta}$, trinodis $\delta$, $q$, se, $f q$.

Short-tongued Bees (22)-Halict.: Agapostemon radiatus $q$, sc, viridulus $\sigma^{\lambda} q$, se, Augochlora viridula + , se, Chloralictus pruinosus $o^{\lambda}$, Curtisapis coriacea $o^{\pi}$, Odontalictus $q$, se, Oxystoglossa pura $q$; Collet.: Colletes armatus $q$, compactus $\delta^{\lambda}+$, fq, eulophi $q$, sc; $D u$ foure.: Halictoides $\sigma^{\top}$; Andren.: Pterandrena aliciae $q$, se, helianthi $\delta$, pulchella $\delta$,, se; Panurg.: Calliopsis andreniformis + , coloradensis $\delta$, sc, in cop, Perdita $\delta$, sc, ab, in cop, Pseudopanurgus compositarum $\delta^{\lambda}$, labrosiformis $q$, se, rudbeckiae $\delta^{\lambda} \uparrow, \mathrm{se}$, ab, rugosus ㅇ, se, solidaginis $\sigma^{\top}$.

Other Hymenoptera (18)-Philanth.: Cerceris prominens, fulvipes; Sphec.: Sceliphron, Sphex gracilis, nigricans; Bembic.: Bembix nubillipennis, spinolae, Bicyrtes 4-fasciata, ventralis; Nysson.: Pseudoplisus divisus; Vesp.: Vespula germanica; Eumen.: 
Ancistrocerus tieris, Leionotus histrionalis; Scoli.: Flis J-cincta, Scolia bicincta ab), Ichneumom.: Ceratosomat, Exetastes suatreolens fq; Bracon.: Aenignostomus.

Diptera (3t) - Syrph.: Eristalis dimidiatus fy, latifrons, transversus, vinetorum, Mesogramma polita i, n, Milesia, ()rthoneura nitida f, n, syrphus americanus i, n; bombyli.: Anthrax haleyon, Exoprosopal decora, fisceiata fip, fascipennis fy, bepidophora, Sparnopolius ab, Systropus in eop., Toxophora; Conop.: Physocephala texana, Robertsonomyia, Zodion fulvilrous; Trachin.: Aetia, Apinops, Arehytas aterrima fy, Leskiomima secunda, Oeyptera tenuis, Peleteria fq, Senotainia trilineata, Siphoplagia anomala fq, SpalIanzania; Dexi.: Ptilodexia, Seotiptera fq; Musc.: Compsomyia, Incilia catesar; Anthomy.: Phorbia platura, Proboscimyia.

Lepidoptera (15) - Vymphal.: Anosia fip, Argynis, Basilarehia archippus, astyanax, Phyciodes, Vanessa eardui fq; Papilion.: Eurymus, Laertias, Nathalis, l'apilio, Pieris; Hesperi.: Epargyreus, Limochores tamas, Thanaos juvenalis; Noctu.: INeliothis armiger.

Coleoptera (6)-Calandry.: Rhodobaenus gn, n; Chrysomel. (f, n) : Chrysomela, Diabrotica 12-punctata; Lampyr.: Chauliognathus pemsylvanicus ab, in eop; $\operatorname{Mclo}(\mathrm{f}, \mathrm{n})$ : Epicauta cinerea, pennsylvanica.

Ilemiptera (1)-Pentatom.: Euschistus variolarius.

Bidens bipinnata.-Blooms August 17-Octoloer 26; 11 visitors observed, August 29 and September 12; Mas, Y.

Long-tongued Bees (6)-Ap.: Apis se, fq ; Bomb.: Bombus amerieanorum ఫ̈; Megachil.: Trypetini: Neotrypetes truncatus q; Megachilini: Anthemois $q$, se, sayapis sayi $q$; Coelioxyini : Coelioxys sayi $\sigma^{\circ}$.

Diptera (4)-Syrph.: Syritta ; Empid.: Parempis; Tachin.: Siphona, Trichophora.

Lepidoptera (1)-Papilion.: Pieris.

Bidens connata.-Blooms September 3-26; 2 visitors observed, September 22 ; Mas, Y.

Long-tongued Bees (2)-Bomb.: Bombus americanorum $\hat{\sigma} \phi$ fq, impatiens $\varnothing$.

Bidens frondosa.-Pluoms Aurust 27-Oetoler 2: 11 visitors observed, August 27 and September 11; Mas, Y.

Long-tongued Pees (2)-Ap).: Apis; Bomb.: Bombus impatiens $\not$.

Short-tonerued Bers (3)-Mulict.: Olontalictus \&. Oxystoglossas confusa $q$; Collet.: Colletes compactus $\widehat{\delta}$. 
Other Hymenoptera (1)—Sphec.: Sphex vulgaris.

Diptera (4) -Syrph.: Tropidia albistylum; Bombyli.: Exoprosopa decora; Tachin.: Peleteria, Spallanzania.

Lepidoptera (1)-Nymphal.: Charidryas.

Bidens laevis (15: 474).-Blooms August 26-October 17; 52 visitors observed, September 20, 22 ; Mas, Y.

Long-tongued Bees (15)-Ap.: Apis ab; Bomb.: Bombias scutellaris $\widehat{\sigma}$, separatus $\widehat{\sigma}$, Bombus americanorum $\widehat{\sigma} \not{\phi}, \mathrm{fq}$, impatiens $\Varangle$; Ceratin.: Ceratina $q$; Megachil.: Megachile brevis $\delta$ q, sc, fq, mendica ơ, petulans ơ, Xanthosarus of, sc; Epeol.: Epeolus autumnalis $q$, type; Eucer.: Melissodes agilis $q$, boltoniae $q$, se, fq, simillima $\sigma$, trinodis ․

Short-tongued Bees (4)-Halict.: Chloralictus versatus $q$, sc, Odontalictus $\sigma^{\lambda}$; Collet.: Colletes compactus $\sigma^{\lambda}$ ㅇ, sc, fq; Andren.: Pterandrena aliciae q, sc, type.

Other Hymenoptera (5)_Vesp.: Polistes annularis, pallipes; Eumen.: Eumenes fraternus; Scoli.: Scolia bicincta fq; Ichneumon.: Exetastes suaveolens.

Diptera (12) - Syrph.: Eristalis dimidiatus ab, transversus ab, Helophilus similis, Mesogramma marginata; Bombyli.: Anthrax halcyon, Sparnopolius fq, Systoechus; Tachin.: Archytas aterrima fq, Siphoplagia anomala; Musc.: Compsomyia fq, Pseudopyrellia fq; Anthomy.: Hydrophoria.

Lepidoptera (14) - Nymphal.: Anosia ab, Basilarchia archippus, Phyciodes, Vanessa atalanta, huntera; Lycaen.: Chrysophanus; $P$ pilion.: Eurymus fq; Hesperi.: Atalopedes, Hylephila; Ctenuch.: Scepsis; Noctu.: Feltia, Heliothis armiger, dipsaceus, Plusia simplex.

Coleoptera (2)-Chrysomel.: Diabrotica 12-punctata $f, n$; Lampyr.: Chauliognathus pennsylvanicus ab.

Boltonia asteroides (15 : 458-9).-Blooms August 8-October 4; 138 visitors observed, August 25-October 4; Mis, W.

Long-tongued Bees (21)-Ap.: Apis se, ab; Bomb.: Bombias

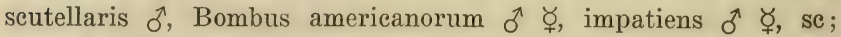
Megachil.: Trypetini: Neotrypetes truncatus $q$, se; Megachilini: Megachile brevis $\delta$, sc, fq, mendica $q, 6$-dentata $q$, se, Xanthosarus $\uparrow$, se; Coelioxyini: Coelioxys 8-dentata $\sigma^{\wedge}$; Nomad.: Holonomada placida $\sigma^{\Uparrow}+$; Epeol.: Epeolus bifasciatus $q$, pusillus $\delta^{\Uparrow}+$, Triepeolus cressonii ơ $q$, fq, pectoralis $\delta q$, fq; Eucer.: Epimelissodes obliqua $q$, se, Melissodes asteris $q$, se, autumnalis $\delta$, se, boltoniae $q$, sc, fq, type, nivea $q$, sc, simillima ơ $q, \mathrm{sc}, \mathrm{ab}$. 
Short-tongmed Bees (20)-Malich: Agapostemon radiatus of Chloralietus obscurus $\delta$, pruinosus $\delta \&$, se, sparsus $f$, se, versatus

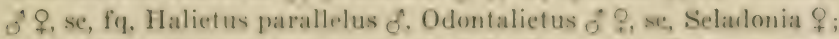
Spherodini : Spheodium eressonii of o; Prosopril.: Prusopis pygmaea of $q$. ab, ziziate of: Collet.: Colletes americamus of $q$, se, ab. eompactus of eulophi $q$; Andren.: P'teraudrena solidaginis \& 9. se, fi], in eop; Penury.: Calliopsis eoloratensis of f. sc, ah, in cop, Perdita of 9. se. fq. Perditella boltoniae of \&. sc. oligolege. type, Pseudopanurgus compositarum of $f$, se, ab, type, solidaginis $f$, se.

Other Hymenoptera (12)-Spher.: Priononyx thomate, Sulhex nigricans, pietipemnis; Bembic.: Bembix spinolae: Scoli.: Seolia bieineta; Philanth.: Cereeris fulvipes; Vesp.: Polistes variatus; Eumen.: Leionotus anormis, elypeatus; Lurr.: Astatus unicolor, Larropsis; Ichneumon.: Ceratosoma.

Diptera $(60) \rightarrow$ Syrph.: Baecha elavata, Eristalis dimidiafus s? latifrons, Mesogramma geminata, marginata sf, pulita f, n, Paragus tihialis sf, Sphaterophoria si, Spilomyia longionmis, 4-fasciata, Syritta sî, ab, syrphus americanus si: Empil.: Parempis; Bumbyli.: Anthrax fulvohirta, Sparnopolius ab, Systocehus, Systropus, Toxophora; Conop.: Oneomyia, Zodion fulvifrons, nanellum, Rolertsonomyia in eop; Tuchin.: Alophora aeneoventris, Arehytas analis, Cistogaster oecidua, pallasii, Epigrimyia floridensis, Euacemyia, Gymnosoma, Hypostena variabilis, Leueostoma atra, neomexieana, Enmyonma globusa, nigrifrons, Oeyptera dosiales. Patehyophthalmus signatus, Paradidyma. Peleteria, Phorantha humeralis, masna, purpuraseens fq. Senotainia rubriventris, trilineata fip, Siphoplagia anomala, Spallanzania li) Deri: Myocera: Surcophay.: Helicohia helicis, Sarcophaga hunteri, sinuata; Musc.: Componyia, Graphomyia, Lneilia sericata, Psondopyrellia, Stomoxys; Anthomy.: Anthomyirlla, Calythea, Limnophora, Probuseimyia; Seps.: Sepsis; Trypet.: 'Trypeta humilis ab.

lepidoptera (20)-Nymphal.: Anosia. Jumonia. Phyciodes, Vanesa eardui, huntera: Lycain.: Chrysophanus. Everes. IIeodes, Uranotes; Puprilion.: Eurymus fu. Pieris. Poutia; Hesperi.: Aneyloxypho. Hesperia, Mylephila, Limolonres taumas, Polites: Arcli.: Utetheisa ; Cenuch.: Seepsis: Noctu.: Heliothis armiger.

Culeoptera $(1)$-Chrysumel. (f, fq. n) : Diabrotien 12-pumetata, longieornis: Coucinell.: Hippodamea parenthesis f, n: Lampyr.: Chauliognathus pennsylvanicus fq.

Iemiptera (1)-Caps.: Lygus fq.

Outuber 15, 17, the following 40 spewies and 186 indiviluals were taken as they eame to the flowers: 
Long-tongued Bees ( $8: 66)-A p .:$ Apis 3; Megachil.: Megachile brevis 3 ; Epeol. Epeolus pusillus 1, Triepeolus cressonii 1; Eucer.: Melissodes asteris 2, boltoniae 2, nivea 3, simillima 51 .

Short-tongued Bees (5:20)-Halict.: Chloralictus versatus 13; Panurg.: Calliopsis coloradensis 1, Perdita 3, Pseudopanurgus compositarum 2, solidaginis 1 .

Other Hymenoptera (1:1)-Eumen.: Leionotus anormis 1.

Diptera (17: 79)—Syrph.: Eristalis latifrons 3, Mesogramma geminata 1, marginata 2, polita 2, Syritta 7; Bombyli.: Sparnopolius 39; Conop.: Zodion fulvifrons 1; Tachin.: Cistogaster occidua 1 , Phorantha humeralis 5, magna 1 , Senotainia rubriventris 5, trilineata 2, Siphoplagia anomala 1, Spallanzania 2; Sarcophag.: Helicobia helicis 1; II usc.: Graphomyia 1; Anthomy.: Limnophora 5.

Lepidoptera (6:9)-Nymphal.: Anosia 1, Phyciodes 3; Papilion.: Eurymus 2; Hesperi.: Hylephila 1, Limochores taumas 1, Polites 1.

Coleoptera (3: 11)-Chrysomel.: Diabrotica 12-punctata 1, longicornis 1 ; Lampyr.: Chauliognathus pennsylvanicus 9 .

Brauneria pallida (15: 465-6).-Blooms May 25-June 30; 38 visitors observed, May 25-June 21; Mas, $R$.

Long-tongued Bees (14)-Ap.: Apis se; Bomb. (q) : Bombias auricomus, separatus, Bombus americanorum, Psithyrus variabilis; Ceratin.: Ceratina $q$, se; Megachil.: Megachilini: Cyphopyga $\delta$, Megachile brevis $\delta+\%$, mendica $q$, Sayapis pugnata $\delta$; Coelioxyini: Coelioxys 8-dentata $\delta^{\top}$; Xylocop.: Xylocopa $q$; Nomad.: Holonomada affabilis $\delta$, superba $q$.

Short-tongued Bees (9)-Halict. ( $($ ) : Agapostemon texanus se, viridulus se, Chloralietus pilosus, pruinosus, versatus se, Odontalictus se, Oxystoglossa confusa, similis; Panurg.: Pseudopanurgus albitarsis 07 , se.

Other Hymenoptera (1)—Sphec.: Sphex nigricans.

Diptera (1)_Tachin.: Spallanzania.

Lepidoptera (12)-Nymphal.: Anosia, Basilarchia archippus, Charidryas, Vanessa atalanta, huntera; Lycaen.: Chrysophanus; Papilion.: Eurymus, Papilio, Pontia ; Hesperi.: Limochores taumas, Polites; Sphing.: Hemaris thysbe.

Coleoptera (1)-Cerambyc.: Typocerus sinuatus $\mathrm{f}, \mathrm{n}$.

Brauneria purpurea (15:466).-Blooms June 16-September 26; 48 visitors observed, July 8-September 14; Mas, R.

Long-tongued Bees (16)-Ap.: Apis fq;Bomb.: Bombias auricomus $\sigma^{\lambda} \not{q}$, seutellaris $\not{q}$, separatus $\delta^{\lambda} \not{q}$, Bombus americanorum 
d. G. impatiens Q. Psithyrus variabilis 9: Coralin. Cerstins Q. se; Meguchil.: O-mini: Ashmeadiella Q. e: Mrgmehilini: Mrcachile

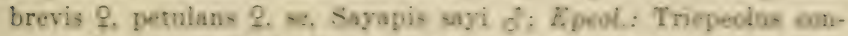

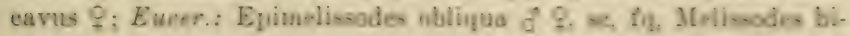
maculata $\sigma^{*}$ \% , enici $ᄋ$.

Short-tongned Bees (7)-Helict.: Apupontemon viridulas of $\mathrm{Q}$. se, Chloralietıs pilisus $9, s c$, sparsd $f, \approx$, vernatus 2 . Bvylarus

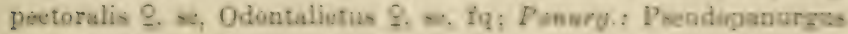
albitarsis $ᄋ$, se.

Other Hymenoptera (3)-Sphec.: Spher niemicus, provera: Larr.: Tachytes aurulentus.

Dipters (9) - Syrph.: Voliaclla vesieulos; Fmpil.: Parempis; Bombyli: Anthrax haleyon, parvicomis, Exoprosopa decora, fas.

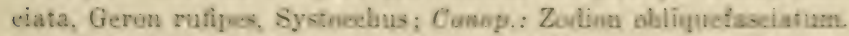

Lepidoptera (13)-Nymphal.: Anoik, Argynnis. Charidryses. Euptoieta, Vanesa cardui, huntera: Popilion.: Euphosades, Eurymus, Jasoniades, Pieris. Pontia: Hesperi: Henperia, Thorybes bathyllus.

Cacalia atriplicifolia.-Blows July 26-5rptember 20; 10 vis:tors observed, August 4 ; Mis, W.

Short-tongued Bees (2. 2, e)-Haliet.: Chloralietils gyarses. Odontalictus.

Other Hymenoptera (7)-Crobron.: Solenius: Orybel.: Nanglosia americana. Oxybelus 4-notatus; Philumth.: Philnothus gunetatus: Sphcc.: Sphex vulgaris; Eumen.: Eumenes fraternus; Soub: Scolia bicincta.

Diptera (1)-Empid.: Parempis ab.

Cacalia reniformis.-Blowms June 25-July 21 ; 39 visitors 6 served, June 25-July 16; Jis, W.

Long-tongued Bees (5)-Ag.: Apis; Cenatin.: Ceratina ?. se. Zaodontomerus d: Megechil.: Neotrypetes truneatus 9, as: Reorr.: Melissodes bimaculata ㅇ.

Short-teugued Bees (11)-Hulet.: Agapnstemon radiatas 6 . Augoehloes viridula 69 . fol. Chloralietus urenonii 6 . sparsns 9 .

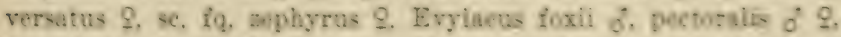
Cxystoglosea yura 9. fq; Presugid.: Prooplis pysuass d. sayi $d$.

Other Hymenopters (11)-Sphec: Splirx macilis nigrieaus. vulearis: Eumen.: Ancistrocerus tigris, fl. Eumces frotemus. Leienotus dorsalls, fundatus fq, perennis, 4-anctus biadoe; Creiren : Solenius.

Diptera (9)-Tigwl: Beranomyis: Syrye: Splanrophoria, 
Syritta $\mathrm{f} q$; Empid.: Parempis fq ; Conop.: Oncomyia, Zodion nanellum; Tachin.: Epigrimyia floridensis, Senotainia trilineata, Siphona fq.

Lepidoptera (1) —Sesi.: Sesia 6-fasciata.

Hemiptera (2)-Caps.: Lopidea fq; Lygae.: Melanocoryphus.

Cacalia tuberosa.-Blooms June 17-July 17 ; 5 visitors observed, July 4 ; Mis, W.

Short-tongued Bees (1)-Halict.: Sphecodes arvensis $0^{1}$.

Other Hymenoptera (1)-Scoli.: Elis 5-cincta.

Lepidoptera (2)-Lycaen.: Chrysophanus ; Ctenuch.: Scepsis.

Hemiptera (1)—Lygae.: Lygaeus.

Chrysanthemum leucanthemum (int.). - Blooms May 24 August 16; 22 visitors observed, May 28-June 21; Mis, W.

Long-tongued Bees (6)-Ceratin.: Ceratina o, se; Stelid.: Chelynia $\delta$, Mierostelis $\delta$, fq; Megachil.: Neotrypetes truncatus $q$, se, Alcidamea simplex $\delta^{\lambda}$; Nomad.: Centrias americanus $\delta^{\lambda} q$.

Short-tongued Bees (3)-Halict. ( $q$, sc, ab): Odontalictus, Oxystoglossa similis; Panurg.: Calliopsis andreniformis $\hat{\jmath}$, ab.

Other Hymenoptera (1)-Eumen.: Leionotus ziziae.

Diptera (11)-Syrph.: Eristalis latifrons, Sphaerophoria, Syritta, Tropidia mamillata; Empid.: Parempis fq, type; Conop.: Zodion fulvifrons; Tachin.: Distichona varia, Leucostoma atra, Phorantha purpurascens ab; Musc.: Pseudopyrellia; Anthomy.: Phorbia platura.

Coleoptera (1)—Scarabaé: Trichius piger $\mathrm{f}$.

Cirsium altissimum (15: 475).-Blooms July 23-October 23; 23 visitors observed, August 14-October 23; Mas, R.

Long-tongued Bees (13)-Ap.: Apis se; Bomb.: Bombias auri-

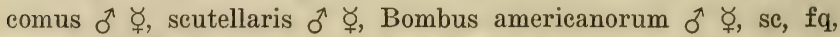
consimilis $\delta^{\lambda} \phi$, se, impatiens $\delta^{\lambda} \not$, se, Psithyrus variabilis $\delta^{\lambda}$; Ceratin.: Ceratina $q$; Megachil.: Neotrypetes truncatus $q$, sc, n; Epeol.: Triepeolus donatus $\sigma^{1}+$; Eucer.: Epimelissodes obliqua $q$, c, Melissodes agilus $\sigma^{\uparrow}$, enici $\delta^{\lambda}+$, se, fq.

Short-tongued Bees (2)-Halict.: Agapostemon viridulus $\delta$, Chloralictus sparsus + , c, n.

Lepidoptera (8)-Nymphal.: Argynnis, Basilarchia archippus, Vanessa atalanta; Papilion.: Euphoeades, Heraclides, Jasoniades; Sphing.: Deilephila, Hemaris thysbe.

Cirsium discolor (51:475).-Blooms July 29-October 20;31 visitors observed, August 21-October 20; Mas, R. 
Long-tongued Bees $(14)-B o m b$.: Bombias auricomus of $q \not \not$, se, fq, seutellaris $\sigma^{8}$. se, separatus of + . Bombus americanorum

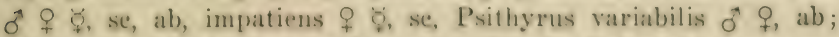
Megachil.: Xanthosarus of $q$, se, iq; Epul.: 'Triepreolus eoneavus

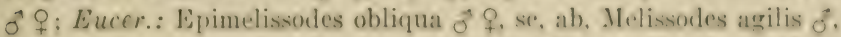
enirei of $q, \mathrm{se}, \mathrm{ab}$, coloradencis ?, se, variabilis $d$; Emphor.: Melitoma $q$.

Short-tonerued Bees (5, ․, e. n)-Ilalict.: (holoraliefus pruinostus, sparsus, versatus, Odontalietus: Collet.: Colletes eulophi.

Diptera (2)-Bombyli.: Exuprosopa iasciata, Systocehus.

Lepidoptera (9)-Nymphal.: Anosia, Aregnnis, Speyeria, Vanessa carelui : P'apilion.: C'allihlyas. Heraclides. Papilio: Sphing.: Deilephila, Hemaris thysbe.

Culeoptera (1)-Chrysomel.: Diabrotica loneicomis $\mathrm{i}, \mathrm{n}$.

Cirsium lanceolatum (int, 15: 475-6i).-. Blooms June 24-Octoher 27: 48 visitors observed, July 3-Oetober 27 ; Mas, R.

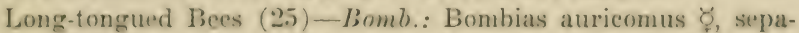

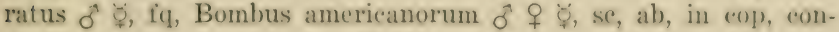

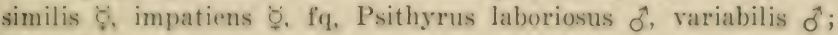
Meguchil.: Megachile 6-dentata of, type, Xanthosarus $q$, se, fq; Epul.: Triepeolus concarus $\vec{o}$. conrolor $\vec{o}$ o. fq, donatus $\vec{\delta}$, neva-

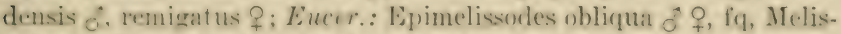

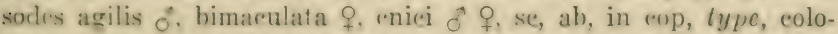

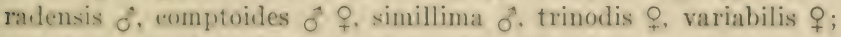
Emphor.: Emphor $\delta^{\star} ;$ Anthophor.: Amegilla $\delta^{\dagger}$.

Short-tongued Bees (6)-Maliel.: Agopostemon radiatus d.

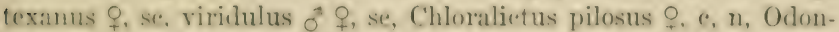
talictus + , e, n N Nomi.: Paranomia $q$.

Diptera (3)-Bombyli.: Exoprosopa iasciata, systoechus fq: Conop.: Physoeephala tibialis.

Lepirloptera (14)-Nymphal: Anosia, Phyciodes, Sipereria: Pupilion.: Euphoeades, Eurymus, Jasoniades, Pappilio, Puntia, Tanthidia; Hesperi: Atrytone zabulon, Limochores taumas $\mathrm{f} q$. Polites, Thorybes bathyllus; Ctenuch.: Seepsis.

Cirsium pumilum-Blooms May 25...June 29: 17 visiturs whserved, May 29, June 26; Mas, R.

Long-tongued liees (4, Q)-Bomb.: Bombus ameriranorum. Psithyms variabilis: Meguchil.: Cyphopyga se, Sayapis juguata se.

Short-tongued Bees (1)-IIalict.: Evylaeus pectoralis 9, c, n.

Lepidoptera (9)- Nymphal.: Anosia, Aregnnis ab, Speyeria: Pupilion.: Euphomales, Euryuus, Heraclides, Laertias; Hesperi.: Polites, Thorybes bathyllus. 
Coleoptera (2, f, n)-Cerambyc.: Typocerus sinuatus; Scarabae.: Trichius piger gn, in cop, ab.

Hemiptera (1)-Lygae.: Lygaeus.

Coreopsis palmata $(15: 472)$.-Blooms June 3-July 24; 116 visitors observed, June 6-July 2 ; Mas, $\mathrm{Y}$.

Long-tongued Bees (33)-Ap.: Apis se; Bomb.: Bombus americanorum $q$ ६; Ceratin.: Ceratina $\uparrow$, se, fq; Stelid.: Anthidium cognatum $0^{\pi}$, Microstelis $q$; Megachil.: Trypetini: Neotrypetes truncatus $\delta^{\lambda} q$, se; Osmiini: Alcidamea simplex $q$, Ashmeadiella $\delta^{\lambda} q$, Gnathosmia + , sc; Megachilini: Chelostomoides $\delta$, Cyphopyga $\delta$, Megachile addenda $\delta$, brevis $\delta$, se, ab, in cop, mendica $\delta^{\lambda}+$, petulans $\sigma^{\lambda}, 6$-dentata $\delta^{\lambda}+$, sc, Sayapis pollicaris $\delta^{\lambda}+$, se, pugnata $\delta^{\lambda}+, \mathrm{sc}$, fq, Xanthosarus $q$; Coelioxyini: Coelioxys 8-dentata $\hat{\delta}$ 이 ab, sayi $\delta q$; Nomad.: Centrias americanus $q$, erigerontis $\delta \uparrow$, Holonomada superba $q$; Pasit.: Holcopasites illinoensis $\sigma^{\tau} q$, fq; Epeol.: Epeolus bifasciatus $\delta^{\lambda}$, interruptus $\delta^{\pi} q$; Melect.: Melecta $\delta^{\lambda}$; Eucer.: Melissodes agilis $\sigma^{\lambda}$, boltoniae $q$, se, coreopsis $\sigma^{\lambda}+, \mathrm{sc}, \mathrm{fq}$, oligolege, type, petalostemonis + , ab, trinodis $q$, sc.

Short-tongued Bees (21)-Halict.: Agapostemon radiatus $\uparrow$, se, texanus $q$, viridulus $q$, Augochlora fervida $q$, Chloralictus coreopsis $\delta^{\lambda} q$, se, fq, type, obscurus $\delta^{\lambda}$, pilosus $\delta^{\lambda}+$, se, fq, pruinosus $\delta^{\lambda} \uparrow, \mathrm{se}$, tegularis $\delta$, versatus $\delta$, se, ab, Evylaeus areuatus $q$, se, foxii $\delta$, pectoralis $\sigma^{\lambda} q$, se, Halictus lerouxii $\sigma^{\lambda}+$, se, Odontalictus $\sigma^{\lambda} q, \mathrm{se}, \mathrm{fq}$, Oxystoglossa confusa $\delta^{\lambda} q$, similis $\delta^{\lambda} q$, se, Seladonia $\delta^{\lambda} q$, se; Andren.: Pterandrena rudbeckiae $\sigma^{\tau}+$; Panurg.: Calliopsis andreniformis $\sigma^{\lambda} q, a b$, Pseudopanurgus albitarsis $\delta^{\lambda}+$.

Other Hymenoptera (14)-Sphec.: Priononyx atrata, thomae, Sphex pictipennis, nigricans, procera, vulgaris; Bembic.: Bembix spinolae, Bicyrtes ventralis, Stictiella; Philanth.: Cerceris prominens; Eumen.: Leionotus anormis, arvensis, histrionalis; Bracon.: Toxoneuron abdominale.

Diptera (30)—Stratiomy.: Odontomya nigrirostris; Syrph.: Eristalis flavipes, latifrons, tenax, transversus, Helophilus latifrons, Mesogramma marginata, Sphaerophoria, Syrphus ribesii; Bombyli.: Anthrax parvicornis, Exoprosopa decora, emarginata, Geron rufipes, Systoechus, Thripsogaster fq, Toxophora; Conop.: Oncomyia, Physocephala tibialis, Zodion fulvifrons; Tachin.: Archytas analis, Euphorocera claripennis, Linnaemyia, Ocyptera lata, Peleteria, Spallanzania; Musc.: Lucilia caesar, Pseudopyrellia, Stomoxys; Anthomy.: Proboscimyia; Agromyz.: Eusiphona.

Lepidoptera (14) - Nymphal.: Argynnis, Phyciodes, Vanessa huntera; Lycaen.: Uranotes; Papilion.: Euphoeades, Eurymus, 
Pieris, Pontia; IIesperi.: Atrytone zabulon, Limochores taumas, Pholisora eatullus, Thanaos juvenalis; Ctenuch.: Seepsis; Noctu.: Plusia simplex.

Coleoptera (4)-Cerambye.: Typoeerus sinuatus I, n; Mclo.: Epieauta cinerea $\mathrm{f}, \mathrm{n}$; Mordell.: Mordella marginata, seutellaris $\mathrm{f}, \mathrm{n}$.

Coreopsis tripteris $(15: 472-3)$.-Blooms July 23-September 26 ; 53 visitors and 185 individuals observed, August 12-September 3 ; Mas, Y.

Long-tongued Bees $(18: 83)-A p .:$ Apis se, $1 ; B o m l$.: Bombas amerieanorum ô $\phi, 13$, impatiens $\phi, 1$; Ceratin.: Ceratina + , se, 5 , Megachil. (q) : Cyphopyga 1, Megachile brevis se, 1, petulans se, 9; Epeol.: Epeolus pusillus $q, 1$, Triepeolus concavus $q$, 1, donatus ‥ 1, lunatus of 1 ; Eucer.: Epimelissodes obliqua $q$, se, 32, Melissoles agilis $\hat{\delta}+2,2$, autumnalis $\hat{\delta}, 2$, coloradensis $q$, se, 1, nivea $\delta, 1$, simillima of $q, 4$, trinodis $\delta, 6$.

Short-tongued Bees (12:31)-Halict.: Augochlora fervida ô, 1, Chloralietus eoreopsis $q$, se, 1 , pilosus $q, 1$, tegularis $q$, se, 1 , versatus $q$, sc, 1 , Curtisapis eoriacea $\delta^{2}, 2$, Evylaeus pectoralis $q, 1$, Odontalictus $q$, se, $13 ;$ Andren.: Pterandrena pulchella of $q$, se, 4; Panurg.: Calliopsis coloradensis $q$, se, 1 , Pscudopanurgus labrosiformis $\widehat{\sigma}+$, type, 2, solidaginis $\hat{\delta}, 3$.

Other Hymenoptera (10:32)-sphec.: Sphex gracilis 3, nigricans 2, pietipennis 2, procera 7 , vulgaris 2 ; Bembic.: Bieyrtes ventralis 2; Scoli.: Seolia bicineta 9; Philanth.: Philanthus punctatus 1; Eumen.: Leionotus anormis 1, arvensis 2.

Diptera $(9: 34)$-Syrph.: Pipiza nigribarba f, 1, Syritta 2; Bombyli.: Anthrax haleyon 4, Lepidophora 1, Sparnopolius 18, Systweehus 4; Conop.: Zodion obliquefasciatum 1; T'achin.: Epigrimyia geniculata 1 , Siphoplagia anomala 2.

Lepidoptera (3:4)-Nymphal.: Anosia 1, Phyciodes 1; IIesperi.: Limochores taumas 2.

Coleoptera (1:1)-Lampyr.: Chauliognathus pennsylvanieus 1.

Erechthites hieracifolia.-Blooms August 20-September 25; 13 visitors observed, August 29-September 16; Mis, Y.

Long-tongued Bees (2) -Ap.: Apis ab; Megachil.: Megachile brevis ㅇ.

Short-tongued Bees (1) - II ulict.: Agapostemon radiatus .

Other Hymenoptera (8)-Sooli.: Elis 5-cineta; Vesp.: Polistes pallipes, variatus, Vespula germanica; Eumen.: Aneistroeerus capra, Ireionotus arvensis, Zethus; Pompil.: Arachnoprortonus. 
Diptera (1) - Tachin.: Archytas aterrima.

Lepidoptera (1)-Lycaen.: Chrysophanus.

Erigeron annuus.-Blooms May 26-September 15; 2 visitors observed, June 2 and 15; Mis, W.

Long-tongued Bees (1)-Megachil.: Neotrypetes truncatus q.

Diptera (1) - Tachin.: Apinops.

Erigeron canadensis.-Blooms August 16-October 29; 18 visitors observed, September 1, October 29; Mis, W.

Long-tongued Bees (1)-Epeol.: Epeolus pusillus ơ.

Short-tongued Bees (1)-Halict.: Chloralictus versatus + , sc.

Other Hymenoptera (7)-Philanth.: Cerceris clypeata, Eucerceris; Vesp.; Polistes variatus; Eumen.: Ancistrocerus tigris; Crabron.: Anacrabro; Larr.: Tachytes elongatus; Ichneumon.: Ceratosoma.

Diptera (9)—Syrph.: Eristalis tenax, Mesogramma marginata, Syritta; Conop.: Oncomyia; Tachin.: Ocyptera lata, Senotainia trilineata; Sarcophag.: Helicobia helicis; Musc.: Musca, Pollenia, Pseudopyrellia.

Erigeron philadelphicus (15:461).-Blooms April 23-June 13 ; 64 visitors observed, May $14-J u n e ~ 5$; Mis, W.

Long-tongued Bees (12)-Ap.: Apis; Bomb.: Bombias separatus + ; Ceratin.: Ceratina $\delta^{\lambda}+$, sc, ab, Zaodontomerus $\delta^{\lambda}, \mathrm{fq}$; Stelid.: Microstelis 0 , fq; Megachil.: Trypetini : Neotrypetes truneatus $\delta^{\lambda}$; Osmiini: Alcidamea simplex ô $q$, Gnathosmia $q$, se; Megachilini: Megachile brevis $\delta$; Nomad.: Centrias americanus $\delta^{\pi}+$, ab, Holonomada superba $\delta^{\lambda}$, Nomada cressonii $\delta^{\lambda}$.

Short-tongued Bees (14)-Halict. (q) : Agapostemon viridulus, Chloralictus albipennis, pilosus se, sparsus, versatus se, fq, Curtisapis coriacea sc, Evylaeus pectoralis sc, fq, Odontalictus sc, ab, Oxystoglossa confusa, similis se, Seladonia se, fq; Sphecodini: Sphecodium eressonii; Prosopid.: Prosopis pygmaea $\sigma^{\lambda} ;$ Andren.: Trachandrena mariae .

Other Hymenoptera (5)-Sphec.: Sphex vulgaris; Eumen.: Leionotus anormis ab, clypeatus ab, foraminatus, illinoensis type.

Diptera (20)—Syrph.: Mesogramma marginata, Paragus bicolor, tibialis, Sphaerophoria; Bombyli.: Aldrichia f, fq, Conop.: Oncomyia, Zodion fulvifrons fq; Tachin.: Apinops atra type, Cistogaster occidua ab, pallasii, Epigrimyia floridensis, Leucostoma atra, Phorantha purpurascens, Siphona; Sarcophag.: Helicobia helicis, Sarcophaga cimbicis, sinuata; Musc.: Pseudopyrellia; Anthomy.: Calythea, Phorbia platura. 
Lepidoptera (7)-Nymphal.: Phyeioles: Lycann.: Chrysophanus; Pupilim. : Xanthidia; IIespuri.: Aneyloxypha, Limochores taumas, Polites; Ctenuch.: Seepsis.

Coleoptera (3, f, n)-Chrysomel.: Diabrotiea 12-punctata; Lampyr.: Telephorus flavipes; Scarubue.: 'Trielius piger ab.

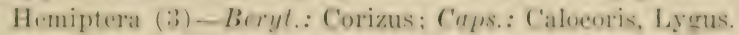

Erigeron pulchellus.-Bloums April 27-June 7 ; 5 visiturs observed, May 4; Mis, W.

Long-tongued Bees (2)-Crratin.: Ceratina of Nomad.: Centrias americanus $q$.

Short-tongued Bees (1)-Mulicl.: Odontalictus ?. se.

Lepidoptera (2)-Lycaen.: Everes; Hesperi.: Thanaos juvenalis.

Erigeron ramosus $(15: 461-2)$.- Blooms May 17-September 15 ; 78 visitors observed, May 31-July 13; Mis, W.

Long-tomgued Bees (12)-Ap.: Apis; Ceratin.: Ceratina of $q$. se, in, Zaodontomerus of stelid.: Microstelis of of iq, Steliclium of. $\mathrm{fq}_{\mathrm{f}}$ Megachil.: Neotrypetes productus of, truncatus of $q$, se, ab: Nomurl. (q) : Centrias americanus, erigeront is, type, Nomada denticulata; Pasil.: Holenpasites illinoensis of $\mathrm{f}$. fy; Ejpol.: Eproolus bifasciatus $\widehat{\sigma}$.

Short-tongurd bees (16)-- Mulirt.: (hloralietus albipennis $q$.

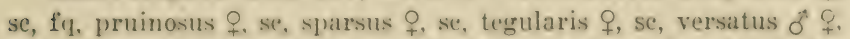
se, zephyrus of. Evylaeus pectoralis q. se, Odontalictus q, se, fq. Oxystoglossil similis O. se, Soladonia . se. $\mathrm{fq}$; Spheeodini : Machaeris stygia 으. Sphecodium eressonii $q$ : Prosopia.: Prosopis eulophi $\hat{o}$. pygmaea , ziziae $q$; Collet.: Colletes eulophi $\sigma^{\star}$.

Other Hymenoptera (10) - Sphec.: Sphex pietipennis; Philanth.: Cereeris elypeata; Eumon.: Leionotus anormis fq. elypeatus, histrionalis, ziziae: Orybel.: Notoglossa frontalis; Chrysid.: Tetraehrysis montana; Chalcid.: Leneospis; Bracon.: Bracon longieauda.

Diptera (36)-Syrph.: Eristalis dimidiatus, latifrons, Mrsogramma geminata, marginata fq. Paragus bieolor, tibialis, Siphaterophoria, Syritta. Tropidia mamillata; Empid.: Parempis: Bombyli.: Bombylius atriepps, Exoprosopa Cascipennis, Onrouluera. Toxoptura: Comop.: Zodion fulvifrons; Tachin.: Apinops, Cistugaster oceidua, pallasii, Epigrimyia polita, Gymnonoma, Leucostoma atra, Oryptera nigra, tenuis, Pachyophthalmus floridensis, Phorantha humeralis $\mathrm{fq}$. purpuraseens, Senotainia rubriventris fq. trilineata, Siphona; Sarcophuy.: Helicohia helieis, Saroophaga eimbicis: Musc.: Psondopyrellia; Anthomy.: Calythen, Limnophora, Phorbia platura; Agromyz.: Lobioptera. 
Lepidoptera (1)-Lycaen:: Chrysophanus.

Coleoptera (2, f, n) -Malach.: Collops ; Mordell.: Mordellistena comata.

Hemiptera (1)-Caps.: Lygus ab.

Eupatorium altissimum.-Blooms July 22-October 5; 79 visitors observed, August 25-September 24; Mis, W.

Long-tongued Bees (9)-Ap.: Apis fq; Bomb.: Bombias scutellaris $\widehat{\sigma}$, Bombus americanorum $q$, impatiens $\widehat{\gamma} \not$; Ceratin.: Ceratina $q$; Nomad.: Nomada vicina $\delta \uparrow$, ab; Epeol.: Triepeolus donatus $\delta$, pectoralis $\delta^{\lambda}$, fq ; Eucer.: Melissodes simillima $\sigma^{\lambda}$ ㅇ, fq.

Short-tongued Bees (13)-Halict.: Augochlora fervida $\sigma^{\lambda}$, viridula $\delta$, Chloralictus illinoensis $\delta$, sparsus $\delta$, versatus $\delta$, zephyrus $\widehat{0}$, Odontalictus $\sigma^{\lambda}+$, Oxystoglossa similis $q$, Seladonia + ; Prosopid.: Prosopis ziziae $q$; Collet.: Colletes americanus $\sigma^{\lambda} ;$ Andren.: Pterandrena asteris $\sigma^{\lambda}+$, fq; Panurg.: Perdita $\sigma^{\lambda}$.

Other Hymenoptera (25)—Sphec.: Isodontia apicalis, Sphex nigricans fq, procera; Bembic.: Bicyrtes ventralis, Megastizus; Scoli.: Elis interrupta, 5 cincta, Scolia bicincta fq; Philanth.: Eucerceris, Philanthus ventilabris; Vesp.: Polistes annularis, pallipes, variatus; Eumen.: Ancistrocerus campestris, tigris, Eumenes fraternus, Leionotus foraminatus, Zethus; Crabron.: Protothyreopus bigeminus; Oxybel.: Oxybelus packardii; Larr.: Larropsis fq ; Pompil.: Lophopompilus philadelphicus, Prioenemoides fulvicornis; Chrysid.: Hedychrum wiltii; Ichneumon.: Ceratosoma.

Diptera (25)—Syrph.: Milesia, Spilomyia longicornis, Syritta; Empid.: Parempis; Bombyli.: Anthrax hypomelas, Exoprosopa fasciata, Sparnopolius, Toxophora; Conop.: Conops xanthopareus, Oncomyia; Tachin.: Actia, Alophora fumosa, Archytas analis, aterrima, Cistogaster pallasii, Leskiomima secunda, Linnaemyia, Ennyomma globosa, Phorantha asteris, Senotainia trilineata, Spallanzania; Musc.: Compsomyia, Graphomyia, Morellia, Pseudopyrellia.

Lepidoptera (6)-Nymphal.: Charidryas, Euphydryas, Phyciodes; Papilion.: Pontia; Hesperi.: Limochores taumas, Polites; Ctenuch:: Scepsis.

Coleoptera (1) -Scarabae.: Euphoria sepulchralis.

Eupatorium coelestinum.-Blooms July 4-September 22; 26 species and 80 individuals observed, September 16, 17, $22 ;$ Mas, R.

Long-tongued Bees (6:11)-Ap.: Apis 1; Bomb.: Bombus americanorum $\Varangle$, se, 3 , Psithyrus variabilis ${ }^{\lambda}, 1$; Ceratin.: Ceratina ㅇ, 1; Megachil.: Megachile mendica $\$$, sc, $3 ;$ Eucer.: Melissodes bimaculata 오, se, 2. 
Short-tongued Bees $(5: 7)$-Maliet.: Angoehlora viridula ?. se. 1, Chloralietus sparsus \&, 1, Qulontalietus of fo, se, 3 , Oxystuglonsat confusa $\sigma^{\top}, 1$, similis $q$, se, 1 .

Other Hymenoptera (1:2)--Sphre.: Sphex nigrieans 2.

Diptera (5:12)-Syrph.: Mesogranma geminata 1 , 4 ; Bombyli.: Sparnopolius 1; Tuchin.: Siphoplagia anomala 5; Deri.: Ptilodexia 1; Muse.: Stomoxys 1.

Lepidoptera $(8: 47)$-Nymphal.: Anosia 3, Basilarehia archippus 1, Phyciodes s; P'apilion.: Eurymus 1:; Mesperi.: Euphyes metacomet 12, Limochores tammas 2. Polites 5; Ctenuch.: Scepsis 4.

Coleoptera (1:1)-Lampyr.: Chauliognathus penusylvanicus 1.

Eupatorium perfoliatum $(15: 453)$.-Blooms July $16-$ Sejtember $23 ; 98$ visitors observed, August $13-$ September $11 ;$ Mis. W.

Long-tougued Bees (6)-Ap.: Apis fq; Bumb.: Bombias seutel-

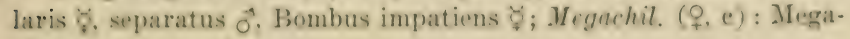
ehile brevis, mendiea.

Short-tongued Bees (8)-Malict.: Augochlora fervida d. Malietus lerouxii of, Odontalietus $\odot$, se, fq, Oxystoglossa similis $q$; Spheeo. dini : Sphecodium pimpinellae o ; Prosopid.: Prosopis pygmaea of. ziziae ơ ; Collet.: Colletes eulophi $\sigma^{\widehat{T}}$.

Other Hymenoptera (4.4)-Sphec.: Ammobia ichneumonea. nuda, Isodontia apicalis, Seeliphron, Sphex gracilis, nigricaus fy, procera, vulgaris; Bembic.: Megastizus; Scoli.: Elis interrupta ab, 5-cineta fq. Seolia bieineta ab; Philunth.: Cerceris elypeata, prominens, Eucerceris ab, Philanthus punctatus; Vesp.: Polistes aunularis, pallipes, rubiginosus, variatus, Tespula germaniea, mateulata ; Eumen.: Ancistrocerus campestris, eapra, tigris ab, Eumenes fraternus, Leionotus anormis, foraminatus; Crabron.: Anaerabro, Hspoerabro, Protothyreopus bigeminus, rufifemur, Solenius fq. Synothyreopus; Oxybel.: Notoglossa americana, frontalis; Larr.: Larropis ab, Tachysphex acutus, Tachytes elongatus: Chrysid.: Iledychrum violaceum, wiltii; Chalcid.: Perilampus cyaneus; Ichneumon.: Ceratosoma, Porizon albipennis.

Diptera (27)-Stratiomy.: Nemotelus; Syrph.: Chilosia punetulata, Mesogramma marginata, polita, Spilomyia longieornis, Syrphus ribesii ; Empril.: Parempis; Bombyli.: Exoprosopa fasriata, Sparnopolius; Conop.: Conops xanthopareus, Oneomyia; Tachin.: Arrhytas analis fq, aterrima, hystrix, Cistogaster awidua, Clytionyia, Eunyomma glohosa, Siphoplagia anomala, Xanthomelana ; Sarcophug.: Ilelieoba helicis, Sarcophaga asidua; Muse.: Compsomyia, Lucilia caesar, Phormia regina, Pseudopyrellia, Stumuxys; Anthomy.: Lispa albitarsis. 
Lepidoptera (7)-Nymphal.: Anosia, Phyciodes; Papilion.: Eurymus; Hesperi.: Aneyloxypha, Pamphila dion; Ctenuch.: Scepsis; Noctu.: Drasteria erechtea.

Coleoptera (6)-Chrysomel.: Ceratoma fq; Curculion.: Centrinus scutellum album; Lampyr.: Chauliognathus pennsylvanicus; Melo.: Epicauta pennsylvanica $\mathrm{f}, \mathrm{n}$; Rhipiphor.: Rhipiphorus limbatus; Scarabae.: Euphoria sepulchralis.

Eupatorium purpureum (15:452).-Blooms July 17-September $3 ; 20$ visitors observed, August 4-September 9 ; Mas, $R$.

Long-tongued Bees (5)-Ap.: Apis $\mathrm{fq} ;$ Bomb.: Bombias auricomus $\not$, Bombus impatiens $\measuredangle$, fq; Eucer.: Melissodes agilis ${ }^{7}$, bimaculata $q$.

Other Hymenoptera (2)-Sphec.: Sphex procera; Vesp.: Polistes rubiginosus.

Diptera (3)—Syrph.: Volucella vesiculosa f ; Bombyli.: Exoprosopa fasciata, Systoechus.

Lepidoptera (10)-Nymphal.: Anosia, Charidryas, Vanessa huntera; Papilion.: Eurymus, Jasoniades, Laertias, Pieris, Pontia; Hesperi.: Pholisora hayhurstii, Polites.

Eupatorium serotinum (15:452-3).-Blooms August 2-October $6 ; 132$ visitors observed, August 14-September 19; Mis, W.

Long-tongued Bees (21)-Ap.: Apis se, ab; Bomb.: Bombias

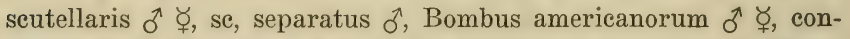
similis $q$, se, impatiens $\delta^{\uparrow}+$, se, fq, Psithyrus variabilis $\delta^{1}$; Megachil.: Trypetini: Neotrypetes truncatus $\delta$ o, se, fq; Megachilini: Megachile brevis $\delta$, mendica $q$, se, Xanthosarus $q$; Coelioxyini : Coelioxys 8-dentata $\delta^{7}$, sayi $\delta$; Epeol.: Epeolus bifasciatus + , Triepeolus cressonii $\delta^{\lambda}+$, lunatus $q$, pectoralis $\sigma^{\lambda}$; Eucer.: Melissodes autumnalis $\sigma^{\top} q$, boltoniae $q$, se, nivea $q$, se, simillima $o^{\top}$.

Short-tongued Bees (11)-Halict.: Agapostemon radiatus $q$,

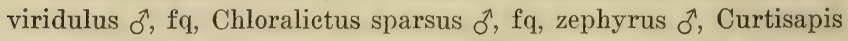
coriacea $\delta$, se, Evylaeus pectoralis $\delta$, Odontalictus $\delta$, Oxystoglossa confusa + ; Sphecodini : Sphecodium pimpinellae $\sigma^{\top}$; Collet.: Colletes compactus $\sigma^{\lambda} q$, eulophi $\sigma^{\lambda} q$, se, fq.

Other Hymenoptera (44) - Sphec.: Isodontia apicalis, macrocephala, Sceliphron, Sphex gracilis, nigricans fq, pictipennis, procera, vulgaris; Bembic.: Bicyrtes 4-fasciata; Scoli.: Elis 5-cincta, Scolia bicincta; Philanth.: Cerceris fulvipes fq, prominens, Eucerceris, Philanthus punctatus, ventilabris; Vesp.: Polistes annularis, pallipes, rubiginosus, variatus, Vespula germanica ; Eumen.: Ancistrocerus campestris, capra, tigris, Eumenes fraternus, Leionotus 
arvensis, dorsalis, foraminatus, serophulariae, Monobia ; Crabron.: Hypocrabro, Protothyreopus bigeminus, P'seudorrabro, Synothyreopus; Larr.: Larropsis ab; Pompil.: Anoplius illinormsis, Pompiloides marginatus; Tiphi.: Tiphia robertsoui; Ohrysid.: Gonochrysis, Hedychrum violaceum, wiltii; Ichneumon.: Cerato. soma, Porizon albipennis; Braeon.: Toxoneuron viator.

Diptera (34)-Tubun.: Chrysopss striatus; Syrph.: Chilusia punctulata, Eristalis tenux, Milesia, Orthomeura nitida, Spilomyia longieornis: Bombyli.: Anthrax alternata, Exoprosopa facriata, faseipennis, Geron rufipes, Sparuopolius, Systreehus, Systropus; Conop.: Conops xinthopareus, Physoeephala trxana, tibialis, stylogaster biannulata; Tachin.: Arehytas aterrima. Belvosia hifasciata, Cistogaster oecidua, Emphoroeera daripennis, Ciymnosnma, Jaskia, Ennyomma nigrifrons, Neofiseheria, Ocyptera tenuis, Siphuplagia anomala, Spallanzania, Trichopoda pennipes, Santhomelana: Sarcophug.: Helicobia helicis; Muse.: Compsomyia, Mnsra, I'sendopyrellia.

Lepidoptera (15)-Nymphal.: Argymis, Phyeiodes, Vamma cardui; Lycuen.: Chrysophanus, Everes; Papilim.: Eurymus. Pieris, Pontia; Hesperi.: Atalopedes, Epargyrens, Limoehores taumas fq; Ctenuh .: Scepsis; Noctu.: Feltia, Spragueia ; Sexi.: Carmenta.

Coleoptera (6)-Cerambyc. (i, n) : Cyllene decorus, robiniae; Chrysomel.: Diabrotica 12-punctata; Lampyr.: Chauliognathus pennylvanicus; Rhipiphor.: Rhipiphorus limbatus; siarahat.: Euphoria sepulchralis.

Hemiptera (1)-Pentatom.: Euschistus variolarius.

Eupatorium sessilifolium.-Blonms August 2-september 11; 8 visiturs observed, August 25, September 11; Mis, W.

Uther Ilynonoptera (6) -Sphec.: Sphex proeera : Scoli.: Seolia bicineta : Philanth.: Philanthus pumetatus fy: Vesp.: Pulistes rubiginosus; Eumen.: Eumenes fraternus, Monobia.

Diptera (1)-Tachin.: Arehytas analis.

Lepidoptera (1)-Lycaen.: Everes.

Eupatorium urticaefolium (15:453).--Bhums July 31-(1.tober $13 ; 23$ visitors observed, September 2-17; Mis, W.

Long-tungued Bees (3) - Ap.: Apis se, ab: Megarbil.: Mecachile mendiea $q$, se, Xanthosarus $q$, e.

Shurt-tongued Bews (4) - IIalicl.: Chloralietus sparsus ?. Odontalietus of P. so. Iq. Oxystoglomsa eunfusa Q. Seladonia o.

Othre Hymenoptera (J)—Seoli,: Soolis bicineta; Vesp.: Vis. fula Germanica, maeulata; Eumen.: Aneistroemrus tigris: L.arr.: Larropsis. 
Diptera (9)—Syrph.: Allograpta, Rhingia, Spilomyia longicornis, Syrphus ribesii; Bombyli.: Exoprosopa fasciata; Tachin.: Cistogaster occidua, Paradidyma; Dexi.: Ptilodexia; Musc.: Pseudopyrellia.

Lepidoptera (2)-Lycaen.: Cyaniris; Ctenuch.: Scepsis.

Gnaphalium polycephalum.-Blooms August 8-October 17; 70 species and 196 individuals observed, September 20-29; Mis, W.

Long-tongued Bees $(1: 1)-A p .:$ Apis 1.

Short-tongued Bees (13:64)-Halict.: Augochlora viridula $q$, 1 , Chloralictus obscurus $\delta^{\top}, 4$, pilosus,+ 1 , sparsus + , 3 , versatus $0^{1}+, 15$, Odontalictus $\delta^{1} q$, se, 11 , Oxystoglossa confusa $q, 1$, similis $\sigma^{\uparrow}+, 8$, Paralictus platyparius ơ $q$, 9, Seladonia + , 2; Prosopid.: Prosopis ziziae,+ 2 ; Collet.: Colletes americanus ô ${ }^{\dagger}, 3$; Andren.: Pterandrena solidaginis ot +4.

Other Hymenoptera (30:63)—Sphec.: Sphex gracilis 1, pictipennis 1 , vulgaris 1 ; Philanth.: Cerceris clypeata 1, compacta 1 , finitima 3, fulvipes 1; Vesp.: Polistes annularis 2, pallipes 1; Eumen.: Leionotus anormis 1, arvensis 1, clypeatus 17, histrionalis 3, illinoensis 1, molestus 1; Crabron.: Ectemnius 1, Solenius 2, Xestocrabro texanus 2, trifasciatus 1; Oxybel.: Notoglossa frontalis 1 ; Larr.: Larropsis 1 ; Pompil.: Lophopompilus philadelphicus 2, Pompiloides americanus 3, marginatus 1, subviolaceus 2; Chrysid.: Gonochrysis 1, Holopyga 3, Tetrachrysis venusta 2; Ichneumon.: Metopius polycinctorius 3; Bracon.: Chelonus 2.

Diptera (22: 54)—Syrph.: Paragus tibialis 1, Syritta 1; Conop.: Oncomyia 1; Tachin.: Distichona varia 3, Euphorocera claripennis 1, Frontina archippivora 1, Gymnosoma 1, Hypostena barbata 1, Ocyptera lata 1, Pachyophthalmus signatus 2, Paradidyma 1, Senotainia rubriventris 1, Siphoplagia anomala 1, Spallanzania 3, Sturmia phyciodis 2, Trichophora 2; Sarcophag.: Helicobia helicis 20, Sarcophaga cimbicis 1; Musc.: Compsomyia 1, Lucilia caesar 2, Musea 1, Pseudopyrellia 6.

Lepidoptera (3:9)-Nymphal.: Phyciodes 3; Hesperi.: Atalopedes 3 ; Ctenuch.: Scepsis 3.

Coleoptera (1:5) -Lampyr.: Chauliognathus pennsylvanicus 5 .

Helenium autumnale (15:474-5).-Blooms August 16-October 22; 35 visitors observed, September 15-23; Mas, Y.

Long-tongued Bees (15)-Ap.: Apis sc; Bomb.: Bombias scutellaris $\delta$, separatus + , Bombus americanorum $\widehat{\delta} \not{\phi}$, fq, impatiens $\sigma^{\lambda} \not{q}, \mathrm{ab} ;$ Megachil. (ㅇ) : Megachilini: Megachile brevis se, ab, mendica se, Xanthosarus; Coelioxyini: Coelioxys 8-dentata; Epeol.: 
Triepeolus cressonii + , ab; Euct.: Epimelissodes obliqua + , Melissodes agilis $\delta$, boltoniae $q$, se, ab, simillima $q$, se, type, trinodis $q$, se.

Short-tongued Bees (3)-IIalict:: Agapostemon viridulus है, Seladonia + , se; Collet.: Colletes compactus $q$, se.

Other Hymenoptera (7)-Sphec: Priononyx atrata, Sphex nigrieans, procera; Bembic.: Bembix spinolae, Bieyrtes ventralis fq ; Vesp.: Polistes variatus, Vespula germaniea.

Diptera (4) -Syrph.: Syrphus areuatus; Bombyli.: Anthrax haleyon, Sparnopolius.

Lepidoptera (4)-Nymphal.: Phyciodes; Pupilion.: Eurymus; IIesperi.: Limochores taumas; Ctenuch.: Seepsis.

Coleoptera (2)-Lampyr.: Chauliognathus pennsylvanicus ab) Melo.: Epicauta pennsylvanica $\mathrm{f}, \mathrm{n}$.

Hemiptera (1)-Pentatom.: Euschistus fissilis.

Helianthus annuus (cult).-Blooms July 10-September 11; 42 species and $4: 34$ individuals observed, July $11-$ September $6 ;$ Mas, I.

Long-tongued Bees $(19: 311)-A p .:$ p is se, $65 ;$ Bomb.: Bombias separatus $\hat{\delta}, 12$, Bombus americanorus ô $q \hat{q}, \mathrm{qe}, 117$, eon-

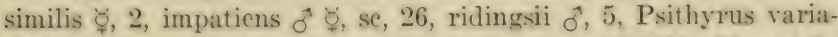

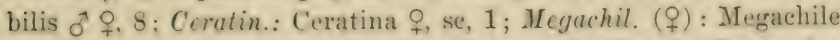
mendiea se, 1, Sayapis sayi 1 , Xanthosarus 1 ; Epeol.: Triepeolus concavus 9,1 ; Eucer.: Epimelissodes obliqua ô $\sigma^{\circ}$, se, 6, Melissodes

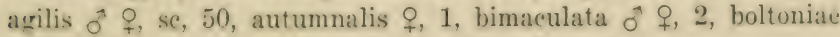
$\sigma^{7}+$, se, 6 , coloradensis $q$, se, 2 , trinodis $\sigma^{7} q$, se, 4 .

Short-tongued Bees (9:69)-Iulict.: Agapostemon viridulus

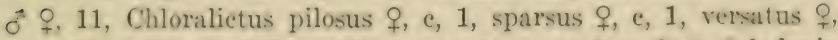
c. 2 , Odontalictus $\delta \mathcal{O}^{\circ}$, se, 50 , Oxystoglossa confusa $q, 1$, Seladonia c. 1 ; Andren.: Pterandrena helianthi ${ }^{*}$, 1 , pulchella $q, 1$.

Dipfera $(5: 36)-$ Syrph.: Eristalis brousi 1, transversus 1, Syritta i, 1, n; Bombyli.: Anthrax alternata 1, 1, u, Sparnopolius 32.

Lepidoptera (4:10)-Nymphal.: Anosia 1, Argynnis 3; Papilion.: Pieris 2; Hesperi.: Limochores taumas 4.

Coleoptera $(4: 5)$-Chrysomel. ( $\mathrm{f}, \mathrm{n})$ : Diabrotima 12-punctata 1, longienrnis 1 , vittata 1 ; Lampyr.: Chauliognathus pennsylvanicus 2.

Hemiptera (1:3)-Caps.: Lygus 3.

Helianthus divaricatus $(10: 244-5)$. Blooms July 22-September $15 ; 75$ visitors observed, July 22-September 10; Mas, Y.

Long-tongued Bees (38)-Ap.: Apis; Bomb.: Bombias setutellaris 8 . se, separatus $\mathcal{C}$, Bombus americanorum of 7. ab; Ceretin.: 
Ceratina $\sigma^{\lambda} q$, se; Megachil.: Trypetini: Neotrypetes truncatus $q$, se; Osmiini: Ashmeadiella $q$, se; Megachilini : Megachile brevis $q$, mendica $\sigma^{\lambda}$, petulans $\delta^{7}+$, sc, fq, 6-dentata + , se, Sayapis pugnata $q$, se, sayi $q, \mathrm{se}, \mathrm{fq}$, Xanthosarus $q$, se; Coelioxyini : Coelioxys alternata + , germana $\sigma^{\lambda}+$, 8-dentata $\sigma^{\lambda}$, sayi $\sigma^{\lambda}$; Epeol.: Epeolus autumnalis $\delta$, $\mathrm{fq}$, bifasciatus $\delta$, pusillus $\delta$, Triepeolus concavus $\delta \phi$, con-

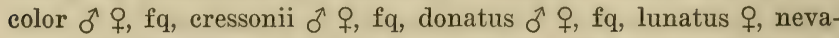
densis $q$, remigatus $\sigma^{\lambda}$, simplex $q$; Eucer.: Epimelissodes obliqua $\delta^{\lambda}+$, se, ab, Melissodes agilis $\delta^{\lambda} q$, se, autumnalis $\delta^{\lambda}+$, se, fq, boltoniae $q$, se, coloradensis $\delta \hat{q}$, sc, fo, nivea $\delta$, fq, simillima $\delta$, trinodis $\hat{o} q, \mathrm{se}, \mathrm{fq}$, vernoniana $\hat{\sigma} q$.

Short-tongued Bees (15)-Halict.: Chloralictus illinoensis $\delta$, pilosus $\sigma^{\lambda}+$, se, versatus $\sigma^{\lambda}$, Evylaeus pectoralis $q$, c, fq, Odontalictus $\delta \uparrow, \mathrm{se}, \mathrm{fq}$, Oxystoglossa confusa $\sigma^{\lambda}+, \mathrm{se}, \mathrm{fq}$; Collet.: Colletes americanus $\sigma^{\top}+$; D D foure.: Halictoides $\sigma^{\lambda}+$, se, fq ; Andren.: Pterandrena helianthi $\delta^{\lambda}$, pulchella $\delta$ o , se, fq; Panurg.: Pseudopanurgus albitarsis $\sigma^{\lambda} q$, se, fq, labrosiformis $\sigma^{\lambda} q$, se, fq, labrosus $\delta^{\lambda}+$, se, fq, rudbeckiae $\delta$, rugosus $\delta$, $\$$ se, fq.

Other Hymenoptera (4)-Sphec.: Sphex nigricans; Bembic.: Stictiella; Eumen.: Leionotus arvensis; Cercer.: Cerceris rufinoda.

Diptera (13)-Syrph.: Eristalis transversus, Milesia f, n; Bombyli.: Anthrax alternata f, n, halcyon, palliata, Exoprosopa decora, Geron rufipes, Sparnopolius ab, Systoechus; Conop.: Zodion fulvifrons fq, Robertsonomyia fq, in cop; Anthomy.: Proboscimyia; Agromyz.: Eusiphona.

Lepidoptera (4)-Nymphal.: Charidryas; Papilion.: Callidryas, Eurymus; Hesperi.: Limochores taumas.

Coleoptera (1)-Lampyr.: Chauliognathus pennsylvanicus fq.

August 27 the following 16 species and 55 individuals were taken on the flowers:

Long-tongued Bees (8:33)-Ap.: Apis 1; Bomb.: Bombus amerieanorum 11; Megachil.: Xanthosarus 4; Epeol.: Triepeolus concavus 1; Eucer.: Epimelissodes obliqua 11, Melissodes agilis 2, coloradensis 1 , trinodis 2 .

Short-tongued Bees (2:2)-Halict.: Chloralictus pilosus 1, Odontalictus 1.

Other Hymenoptera $(1: 1)$-Sphec.: Sphex nigricans 1.

Diptera (2:8)-Bombyli.: Sparnopolius 5, Systoechus 3.

Lepidoptera (2:4)-Papilion.: Eurymus 2; Hesperi.: Limochores taumas 2 .

Coleoptera (1:7)-Lampyr.: Chauliognathus pennsylvanicus 7 .

Helianthus grosse-serratus (15:469-70).-Blooms August 19October 15; 72 visitors observed, August 27-October 15; Mas, Y. 
Long-tongued bees $(29)-A p$. Apis ab: $B(m b)$.: Fombias aurieomus $\delta \phi$, seutellaris $\& \delta$. fq. separatus of $\sigma$, Bombus amerieanorum of $\delta$, ab, consimilis $d$. impatiens $? \&$ Psithyrus variabilis d. fq: Meguchil.: Megachilini : Megachile brevis \& \&. se, wenerosa 9. Sayapis sayi ?. se, Xanthosarus d 9 . se, ab: Corlioxyini : Coelioxys rufitarsis $q$, fq ; Nomud.: Holonomada vineta 6 \& fy; Epeol.: Triepeolus eoneavus \&. fq. eressonii \&. fq, donatus \& \&. helianthi d 2. ab, type; Eucer.: Epimelissodes obliquai of ? se, fq, Melissodes agilis of ?, sc, ah, autumnalis of F, se, fq, bimaculata P. se, boltoniae 9. se. fq, eoloradensis \&, se. fq, nivea of ?, pallida \&. type, simillima $\delta$, trinodis ơ $q, \mathrm{se}, \mathrm{fq}$, type; Anthophor.: Amegilla + .

Short-tongued Bers (3)-Maliet.: Agapostemon radiatus of ? se, texanus of viridulus of, Chloralictus versatus of e, Odontalictus

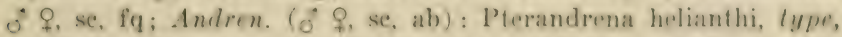
pulehella; Punurg.: Pseudopanurgus solidagiuis of $q$, se, $f(q$; Dufoure.: Halictoides ơ o, se, fq.

Other Hymenoptera (3) - Sphec.: Priononyx atrata; Bembic.: Bembix nubillipennis, spinolae.

Diptera (14)-Syrph .: Eristalis latifrons ab, tenax, transversus ab. Ifelophilus latifrons. similis. Syrphus americanus, ribesii; Bombyli.: Exoprosopa decora, Sparnopolius ab, Systoechus fq; Conop.: Robertsonomyia; Tuchin.: Maequartia; Musc: P'seudopyrellia; Anthomy.: Proboscimyia fq.

Lepidoptera (13) - Nymphal.: Anosia fq, Argynnis, Euptoieta, Speyeria, Vanessa cardui fq, huntera ; Pupilion.: Eurymus, Laert ias, Zerene; Hesperi.: Hespuria : Arcti.: Utetheisa ; Clenuch.: Suepsis; Noctu.: Plusia simplex.

Culeoptera (3)-Chrysomel. (f, n): Diabrotica 12-punctata. lonericornis; Lampyr.: Chauliognathus pennsylvanicus.

Hemiptera (1) - Phymat.: Phymata, pred.

Helianthus laetiflorus.-Blooms August 1-September 15; 26 visitors observed, August 19, 20, 28; Mas, Y.

Long-tongned Bees (9)-Bomb.: Bombias seutellaris 6 , separatus $\delta$. Bombus americanorum $\varnothing ;$; Meguchil.: Megachile brevis of, Sayapis sayi \&. se. Xanthosarus \&: Epeol.: Triepeolus concavus A 9 ; Eucer.: Ep pimelissodes ohliqua d? se, Melisodes agilis d ?.

Short-tongued Beess (4. P, se)-Halict.: Chloralietus pilosus. versatus, Evylacus pectoralis, Odontalictus ab.

Other Hymenoptera (2)-Sphec. : Sphex nigrienus: Ieh neumon. : Ceratosoma.

Diptera (6)-Syrph.: Eristalis latifrons sf, transwersus i: Bombyli: Exoprosopia fasciath, Sparopgolius ab, Systoechus: Tachin.: Siphoplagia anomala. 
Lepidoptera (3)-Nymphal.: Phyciodes; Hesperi.: Pholisora catullus; Ctenuch.: Scepsis.

Hemiptera (2)-Caps: Calocoris, Lygus.

Helianthus mollis (15:469).-Blooms July 9-September 25; 27 visitors observed, July 29-August 23; Mas, Y.

Long-tongued Bees (12)-Bomb.: Bombus americanorum $\delta^{\lambda} \not{\phi}$, fq; Megachil.: Megachilini: Megachile brevis ${ }^{\lambda}$, petulans + , 6-dentata $\delta^{\lambda}$, Xanthosarus $\delta^{\lambda}+$, sc; Coelioxyini: Coelioxys 8-dentata $\delta^{\lambda}$; Epeol.: Triepeolus concavus $q$, cressonii $q$; Eucer.: Epimelissodes

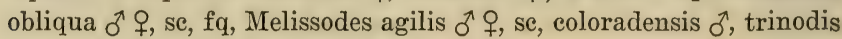
ㅇ, sc.

Short-tongued Bees (7)-Halict.: Agapostemon viridulus $\delta^{7}$, Chloralictus pilosus $q$, se, versatus $q$, se, Odontalictus $q$, se, fq, Oxystoglossa similis $ᄋ$, se; Panurg. ( $\sigma^{\pi}+$, se) : Pseudopanurgus albitarsis in cop, rugosus.

Diptera (6)-Syrph.: Eristalis latifrons, Tropidia mamillata; Bombyli: Anthrax halcyon, Exoprosopa decora, Systoechus fq.

Lepidoptera (2)-Nymphal.: Phyciodes; Papilion: Eurymus.

Helianthus scaberrimus.-Blooms August 2-September 9; 12 visitors observed, August 9-September 9; Mas, Y.

Long-tongued Bees (5)-Bomb.: Bombus americanorum $\Varangle$; Megachil.: Xanthosarus $q$; Eucer.: Epimelissodes obliqua $\delta^{\lambda} q$, Melissodes agilis $q$, se, coloradensis $q$, se.

Short-tongued Bees (3)-Dufoure.: Halictoides + , sc ; Andren.: Pterandrena helianthi $\delta^{7}+$, se, fq, pulchella + , se, type.

Diptera (2)-Syrph.: Eristalis transversus; Bombyli.: Sparnopolius.

Coleoptera (1)-Lampyr.: Chauliognathus pennsylvanicus.

Hemiptera (1)-Caps.: Calocoris.

Helianthus strumosus $(15: 470)$.-Blooms July 6-September 7 ; 14 visitors observed, August 22, 24 ; Mas, Y.

Long-tongued Bees (6)-Bomb.: Bombias separatus ô'; Ceratin.: Ceratina \%; Megachil.: Megachile petulans ô; Epeol.: Triepeolus concavus + , cressonii $\sigma^{\star}$; Eucer.: Epimelissodes obliqua $\sigma^{\lambda}+$, se, fq.

Short-tongued Bees $(2, q)-$ Andren.: Pterandrena pulchella fq ; Panurg.: Pseudopanurgus labrosiformis.

Other Hymenoptera (1)—Sphec.: Sphex nigricans.

Diptera (3)-Bombyli.: Anthrax halcyon, Sparnopolius, Systoechus.

Lepidoptera (2)-Nymphal.: Charidryas; Papilion.: Eurymus.

Helianthus tuberosus $(15: 471)$.-Blooms July 15-September 28; 53 visitors observed, August 13-September 28; Mas, Y. 
Long-tongued Bees $(17)-B$ omb.: Bombias auricomus ti sepa-

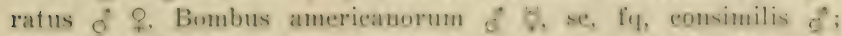
Meguchil. (f): Megachile brevis, Sayapis sayi, se, Xanthosarus; Vomud.: Holonomada vineta $f$; Epeol.: Epeolus autumnalis of ?.

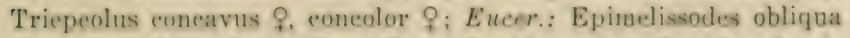
f. se, ab, Melissodes agrilis of $f$. se, fq, bimateulata ?. eoloradensis

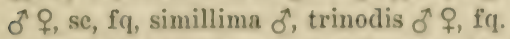

Short-tongued Bees (15)-Haliet. (R): Chloralietus pilosus, sparsus, e, n, zephyrus e, Curtisapis eoriacea se, Evylaeus peetinatus type, Odontalictus se; Collut.: Colletes compactus \& se; In foure: IIalictoides, f; Andren.: Pterandrena alieiae of, se, helianthi \&, pulchella of $q, \mathrm{se}, \mathrm{ab}$; Ponurg.: Pseudopanurgus labroxiformis of $\rho$, se, $\mathrm{f} q$, labrosus $\delta \%, \mathrm{se}, \mathrm{ab}$, in eop, type, rugosus $\delta \%$, se, ab, in erp, solidaginis $\sigma^{\top}$.

Other Hymenoptera (3)-Scoli.: Scolia bicineta; Philun'h.: Cereeris kennieottii; Cralron.: Protothyreopus bigeminus.

Diptera (9)-Syrph.: Eristalis tenax fs, transversus; Bombyli.: Anthrax haleyon fq. Exoprosupa decora, faseipennis fq, Sparnopolius ab, Systoechus fq; Muse.: Pseudopyrellia; Trypet.: Trypeta finalis $\mathrm{n}$.

Lepidoptera (4)-Nymphal.: Phyeiodes: Lycaen.: Eupsyche; Papilion.: Eurymus; IIesperi.: Pholisora catullus.

Coleoptera (4)-Chrysomel.: Diabrotical longicornis $f, n$; Lempyr.: Chauliognathus pennsylvanieus; Melo.: Epieauta pennsylvanica $\mathrm{f}, \mathrm{n}$; Mordell.: Mordella seutellaris $\mathrm{f}, \mathrm{n}$.

Hemiptera (1)-Caps.: Lygus.

Heliopsis helianthoides $(16: 177)$.-Blooms June 22-1)ctuber 2 ; 51 visitors observed, July 1-October 2; Mas, Y.

Long-tongued Pers $(19)$-Bomb.: Bombus amerieanorum d. impatiens \$: Cerutin.: Ceratiua क; Megachil.: Megachilini: Megaehile brevis of fq, 6-dentata of; Coelioxyini : Coelioxys germana of 8-dentata of Q. sayi of Pasit.: Holeopasites heliopsis o. type; Epeol.: Epeolus bifaseiatus $\delta$. fq. Triepeolus eoneavus of $q$, concolor $\delta$ ? fq. cressonii of lunatus f; Euctr.: Epimelissodes obliqua of f. sc. Melissodes coloradensis of. simillima of, trinodis of q. $^{2}$. vernoniana $\widehat{\sigma}$.

Short-tongued Bees (11)-Malict. (९): Chloralietus chiseurus. pilosus se, pruinosns se, sparsus e. n, Evylaens peeteralis e, n. Odontalietus, Oxystoglossa coufusa, pura; Andren.: Pterandrena aliciae \&: Ponurg.: Psoudopanurgus labrosiformis $\delta$ ?, se, rugusus $\widehat{o}, \mathrm{fq}$.

Other Hymenoptera (4)-Sipher.: Sphers nigrieans, vulgaris; Bembic.: Bembix spinolae; Scoli.: Seolia bieineta. 
Diptera (11)—Syrph.: Eristalis transversus; Empid.: Parempis fq; Bombyli.: Anthrax halcyon, Exoprosopa fasciata, fascipennis, Geron calvus fq, rufipes, Sparnopolius, Systoechus; Conop.: Stylogaster biannulata; Agromyz.: Eusiphona.

Lepidoptera (4)-Nymphal.: Phyciodes; Lycaen.: Uranotes; Papilion.: Pontia; Hesperi. : Pholisora catullus.

Coleoptera (1)-Lampyr.: Chauliognathus pennsylvanicus.

Hemiptera (1) - Lygae.: Lygaeus.

Krigia amplexicaulis $(15: 476)$.-Blooms May 1-June 19;68 visitors observed, May 10-June 15; Mis, Y.

Long-tongued Bees (17)-Ap.: Apis sc, ab;Bomb. () : Bombias scutellaris, separatus, Bombus americanorum; Ceratin.: Ceratina ơ ㅇ, se, ab; Stelid.: Microstelis ô; Megachil.: Osmiini : Alcidamea simplex $\delta$ \& fq, Gnathosmia $q$, se, Osmia pumila $q$; Megachilini: Megachile brevis $\sigma^{\lambda}$; Coelioxyini: Coelioxys 8-dentata $\delta^{\lambda}$ q ; Nomad.: Centrias americanus $\delta \mathcal{\delta}, \mathrm{fq}$, Holonomada affabilis $\delta^{\lambda}+$, fq, superba o ab, Nomada illinoensis + ; Epeol.: Epeolus interruptus ơं; Eucer.: Tetralonia dilecta $\sigma^{\wedge}+$, fq.

Short-tongued Bees (16)-Halict. (q): Agapostemon viridulus se, Chloralictus albipennis se, ab, coreopsis se, pilosus se, ab, versatus se, ab, Evylaeus pectoralis se, Halictus lerouxii, parallelus, Odontalictus se, ab, Oxystoglossa confusa se, ab, similis se, ab,

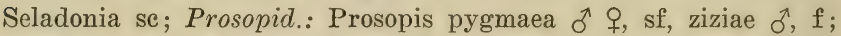
Collet.: Colletes aestivalis $\delta$ o; Andren.: Pterandrena krigiana $\delta$ ㅇ, se, ab, type, oligolege.

Other Hymenoptera (9)—Sphec.: Sphex pictipennis, vulgaris; Eumen.: Leionotus anormis ab, clypeatus, foraminatus, histrionalis, scrophulariae, ziziae; Crabron.: Solenius.

Diptera (17) -Syrph.: Eristalis dimidiatus ab, latifrons, tenax, transversus, Helophilus latifrons, similis, Mesogramma marginata, Sphaerophoria, Tropidia mamillata; Conop.: Oncomyia, Zodion fulvifrons; Tachin.: Cistogaster occidua, pallasii, Ocyptera tenuis; Musc.: Lucilia sylvarum, Pseudopyrellia; Anthomy.: Phorbia acra.

Lepidoptera (5)-Nymphal.: Anosia, Phyciodes; Papilion.: Eurymus, Papilio; Hesperi.: Polites.

Coleoptera (3, f, n)-Chrysomel.: Diabrotica 12-punetata fq; Coccinell.: Megilla; Erotyl.: Languria fq.

Hemiptera (1)—Lygae.: Lẏgaeus.

May 18 and 25, the following 33 species and 202 individuals were taken on the flowers:

Long-tongued Bees $(5: 17)-A p .:$ Apis $11 ;$ Bomb.: Bombias separatus 1, Bombus americanorum 1; Ceratin.: Ceratina 3 ; Eucer.: Tetralonia dilecta 1 . 
Short-tongued Beres $(13: 109)$ - Maliet.: Agapustemon viridulus 3. Chloralietus eoreopsis 1, pilosus 18, versatus 32, Evylarus jeetoralis 2. Halictus Lerouxii 3 , parallelus 1 , Odontalietus 10. Oxystoglossa confusa 10, similis 13; P'rosopill.: I'rnsupis pygruaea 1; Collel.: Colletes aestivalis 4; Andren.: Pterandruna Erigiana 11.

Diptera (11: 72 )-Syrph.: Eristalis dimidiatus 8 , latifrons 1. Helophilus latifrons 1 , similis 1, Mesogramma marginata 34. Sphateroporia 3: Comop.: Onenmyia 2, Zodion fulvifrons 2-: Tuchin.: Cistugaster pallasii 1; Muse: Lucilia sylvarum 1; Anthomy.: Phorbia acra 18.

Lepidoptera (2: 2)-Nymphal.: Plıyeiodes 1; Papilion.: Eurymus 1 .

Coleoptera (1:1)-Chrysomel.: Diabrotica 12-punctata 1.

IIemiptera $(1: 1)$ - Iyygae.: Lygaeus 1.

Lactuca canadensis.-Blooms Jume 2--S-September 6; 1 visitor observed, August 30; Mas, $\mathrm{Y}$.

Long-tongued Bees (1) -Meguchil.: Xanthusitus ․ se.

Lactuca floridana.-Blooms July 25-Oetober 6; 15 visitors observed, August 17-October 6; Mas, R.

Long-fongued Bees (S)-Ap.: Apis se, fq; Bomb.: Bombias aurieomus $\varnothing$. Bombus impatiens $\zeta$, se; Ceratin.: Ceratina $q$; Megachil.: Megachile brevis $\sigma^{\circ} \rightarrow$, se, Xanthosarus ${ }_{+}$; Eucer. $(q$, se) : Epimelissodes obliqua fq, Melissodes boltoniae.

Short-tongued Bees $(6, q)$ - Malicl.: ('hloralictus pilosus c, n, sparsus e, n, Evylaeus peetoralis e, n, Odontalietus se; Prosopid.: Prosopis ziziae, $\mathrm{f}, \mathrm{n}$; Collet.: Colletes americanus.

Coleoptera (1)-Lampyr.: Chauliognathus pemsylvanious iq.

Lepachys pinnata $(15: 46-9)$.-Blooms June 19-August 29; 67 visitors observed, July 1 -August 21 ; Mas, Y.

Long-tongued Bees $(27)$-Bomb.: Bombias sentellaris f; Megrehil.: Usmiini: Mleidamea simplex o; Merachilini: Merachile

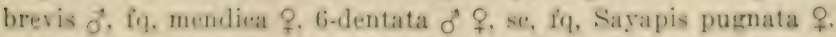
sayi $d$ \&. ab, Xanthosarus of $\&$; Coelioxyini: Coelioxys alternata d f. molesta 6. 8-dentata of f; Eprol.: Epreolus bifasciatus 6, iq. Triepoolus roucavus of $q$. ab, coneolor of $q$, ab. eresonii of $q$, iq. lunatus of \&. fq. remigatus of \&, ab: Eucr.: Epimeli-wodes illinoensis of ?. se, type, obliqua of ?. st, ab, Melissodes agilis $d$ ?. fq. bimaculata of $q$, ab, holtoniae $f$, comptoides $\delta$ ? nivea $\delta$, simillima

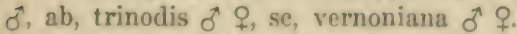

Short-tongued Fees (11)-Matiel.: Agapostemon radiatus $d$.

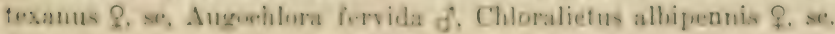


pruinosus $\delta \mathcal{q}$, versatus $q$, se, Curtisapis forbesii $q$, Halictus lerouxii $q$, se, Odontalictus $\delta^{\lambda} q$, se, ab; Andren.: Pterandrena rudbeckiae + , sc; Panurg.: Pseudopanurgus albitarsis $q$.

Other Hymenoptera (16)-Sphec.: Priononyx atrata, thomae, Sphex nigrieans, pictipennis; Bembic.: Bembix nubillipennis ab, spinolae, Bicyrtes ventralis fq, Stictiella fq; Vesp.: Polistes variatus; Eumen.: Leionotus anormis, arvensis, clypeatus, fundatus, Pterochilus; Crabron.: Anacrabro, Entomognathus.

Diptera (8)-Empid.: Parempis; Bombyli.: Exoprosopa decora, Geron rufipes, Sparnopolius, Systoechus; Conop.: Zodion obliquefasciatum fq, in cop; Tachin.: Cistogaster occidua; Agromyz.: Eusiphona.

Lepidoptera (3)-Nymphal.: Phyciodes; Lycaen.: Everes, Uranotes.

Coleoptera (2)-Buprest.: Acmaeodera pulchella f, fq; Cerambyc.: Typocerus sinuatus $f, n$.

Liatris pycnostachya (15:454).-Blooms July 7-August 23; 34 visitors observed, July 24-August 10 ; Mas, $\mathrm{R}$.

Long-tongued Bees (14)-Ap.: Apis ab; Bomb.: Bombias separatus $\delta^{\lambda} \not{\psi}, \mathrm{fq}$, Bombus americanorum $\delta^{\lambda} \not{\zeta}, \mathrm{se}, \mathrm{fq}$, consimilis $\not{\psi}$, impatiens $\not$, se ; Ceratin.: Ceratina $q$, fq ; Megachil.: Sayapis sayi $q$, se, Xanthosarus $\sigma^{\top}+$, fq; Epeol.: Triepeolus concavus $\sigma^{\lambda} q$, concolor, + , remigatus $q$; Eucer.: Epimelissodes obliqua $\hat{\delta}$ ㅇ, se, fq, Melissodes agilis $\sigma^{\pi} q, \mathrm{fq}$, coloradensis $\delta^{\pi}$.

Short-tongued Bees (2)-Halict.: Agapostemon viridulus $\delta$, se, fq, Odontalictus $q$, c, fq, $\mathrm{n}$.

Diptera (6)-Syrph.: Eristalis latifrons, tenax; Bombyli.: Exoprosopa fasciata ab, Systoechus; Conop.: Physocephala texana, Stylogaster biannulata.

Lepidoptera (12)-Nymphal.: Anosia, Basilarchia archippus, Speyeria; Papilion.: Eurymus fq, Laertias, Papilio, Pieris; Hesperi.: Epargyreus, Euphyes metacomet, Limochores taumas fq, Polites fq; Ctenuch.: Scepsis.

Liatris scariosa.-Blooms July 27-October 6; 9 species observed, August 22-September 3; Mas, R.

Long-tongued Bees (2)-Bomb.: Bombus americanorum $\delta$ ఫ; Eucer.: Melissodes nivea ô.

Other. Hymenoptera (1)—Sphec.: Sphex vulgaris.

Diptera (1)-Bombyli.: Exoprosopa fasciata.

Lepidoptera (5)—Nymphal.: Anosia; Papilion.: Euphoeades, Eurymus, Zerene; Hesperi.: Polites. 
Parthenium integrifolium (15: 46t-5).-Bleoms June 6-July 30 ; 55 visitors observed, June 21, 27, July 11 ; Mis, W.

Long-tongued Bees (4)-Ceratin.: Ceratina o, se; Megachil.: Neotrypetes productus o ${ }^{3}$. truneatus o. se; Cyphopyga \&, c.

Short-tongued Bees (7)-Hulict.: Augoehlora fervida f, Chlor-

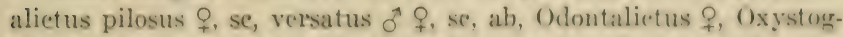
lossa similis $q$, se, fq. Seladonia $q$, se; Prosopid.: Proposis pygmaca $\sigma^{\top}$.

Other Hymenoptera (15) - Sphec: Sphex nigrieans; Scoli.: Elis 5-eincta; Philenth.: Cereeris compacta, robertsonii; Eumru.: Leionotus anormis, elypeatus, histrionalis, ziziae; Crabron.: Solenius; Orybel.: Notoglossa amerieana, frontalis fq ; Chrysid.: Hedychrum parvum; Bracon.: Agathis vulgaris, Vipio; Tenthredin.: Hylotoma humeralis.

Diptera (20)-Syrph.: Mesogramma geminata, marginata, Sphaerophoria, Syritta; Empid.: Parempis; Conop.: Zodion fulvifrons: Tachin.: Cistogaster oceidua, Macquartia, Oeyptera tenuis, Phorantha purpuraseens, Senotainia rubriventris, trilineata $\mathrm{fq}$; Sarcophag.: IIelicobia helieis, Sareophaga hunteri; Muse.: Lueilia sylvarum, Pseudopyrellia, Stomoxys; Anthomy.: Anthomyiella, Calythea; Agromyz.: Lobioptera.

Coleoptera (6)-Ccrambyc.: Typocerus sinuatus fq; Chrysomel.: Diabrotiea atripennis, 12-punetata f; Mordell.: Pentaria; Rhipiphor.: Rhipiphorus limbatus; Scarabae.: Trichius piger sf, fu.

Hemiptera (3)-Caps.: Calocoris, Lygus fq; Corimelaen.: Corimelaena pulicaria.

Prenanthes aspera.-Blooms August 29-Oetober 6; one visitor observed, September 13; Mas, W.

Long-tongued Bees (1)-Bomb.: Bombus amerieanorum $\delta$.

Prenanthes crepidinea.-Blooms August 20-September 25; five visitors observed, August 25, September 6, 13; Mas, Y.

Long-tongued Bees (2)-Bomb.: Bombus amerieanorum $\delta$, impatiens $\varnothing$, se.

Short-tongued Bees (1)-Halict.: Oxystoglossa confusa f. e, n.

Coleoptera (2)-Chrysomel.: Diabrotica longicornis f, fq, 1 ; Lampyr.: Chauliognathus pennsylvanicus $\mathrm{fq}$.

Rudbeckia hirta (15:466-7).-Blowms June 1-September 16; 84 visitors observed, June 9-August 22; Mis, Y.

Long-tongued Bees (14)-Ap.: Apis: Coratin.: Crratina o. sc. fq; Mrgachil.: Trypetini : Neotrypetes truncatus \& se; Megaehilini : Cyphopyga f, se, Megachile brevis of of 6-dentata fi, se, Sayapis 
pugnata $\uparrow$, se; Coelioxyini: Coelioxys 8-dentata $0^{\wedge}$; Nomad.: Cen-

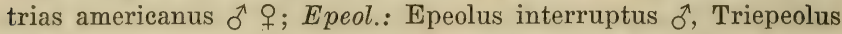
concolor $\delta^{\top}$; Eucer.: Epimelissodes obliqua $\delta^{\lambda}$, Melissodes boltoniae ㅇ, se, fq, trinodis $q$.

Short-tongued Bees (13)-Halict.: Agapostemon radiatus $\delta^{\lambda}$, viridulus $q$, se, Chloralictus coreopsis + , c, pilosus $\sigma^{1}+$, se, pruinosus $\delta^{\lambda}$, versatus $\delta^{\lambda}+$, se, Evylaeus pectoralis $\sigma^{\lambda}+, \mathrm{sc}, \mathrm{fq}$, Odontalictus \%, se, Oxystoglossa confusa + , se ; Collet.: Colletes eulophi + , sc; Andren.: Pterandrena rudbeckiae $\delta^{\lambda}+$, sc, ab, in cop, type; Panurg. $\left(\sigma^{\uparrow} q, \mathrm{se}, \mathrm{ab}\right)$ : Pseudopanurgus albitarsis in cop, rudbeckiae.

Other Hymenoptera (7)-Sphec.: Priononyx thomae, Sphex nigricans fq, pictipennis; Bembic.: Stictiella; Eumen.: Leionotus anormis, arvensis ; Bracon.: Aenignostomus fq.

Diptera (31)—Stratiomy.: Odontomyia nigrirostris; Syrph.: Allograpta, Eristalis aeneus, dimidiatus, latifrons ab, transversus ab, Helophilus latifrons, Mesogramma marginata ab, Paragus tibialis sf, Sphaerophoria, Syritta, Tropidia mamillata, Volucella fasciata; Empid.: Parempis; Bombyli.: Anthrax parvicornis, Exoprosopa decora, emarginata, fascipennis, Geron calvus $f q$, Phthiria cincta fq, sulphurea, Sparnopolius; Conop.: Zodion fulvifrons; Tachin.: Archytas analis, Ocyptera argentea, tenuis ab, Spallanzania fq, Trichophora; Anthomy.: Coenosia antica, Proboscimyia; Sapromyz.: Sapromyza vulgaris.

Lepidoptera (12)-Nymphal.: Argynnis, Basilarchia archippus, Charidryas, Phyciodes; Lycaen.: Chrysophanus, Thecla calanus; Papilion.: Eurymus, Pieris; Hesperi.: Limochores taumas, Polites; Ctenuch.: Scepsis; Noctu.: Plusia simplex.

Coleoptera (6)-Buprest.: Acmaeodera pulchella f; Cerambyc.: Typocerus sinuatus sf, fq; Chrysomel.: Diabrotica 12-punctata f; Curculion.: Centrinus scutellum-album f; Mordell.: Mordella scutellaris $\mathrm{f}$; Melo.: Macrobasis $\mathrm{f}$.

Hemiptera (1)-Caps.: Lygus.

Rudbeckia laciniata (16:177-8).-Blooms July 17 -October 3 ; 52 visitors observed, August 13-October 3; Mas, Y.

Long-tongued Bees (18)-Ap.: Apis se, ab;Bomb.: Bombias

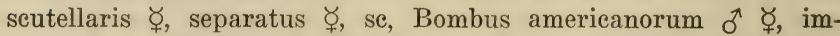
patiens $\delta \not$, Psithyrus variabilis $q$; Ceratin.: Ceratina $q$, Zaodontomerus $\sigma^{\lambda} ;$ Megachil.: Neotrypetes truncatus $\sigma^{\lambda}$; Megachile petulans $q$, se; Epeol.: Epeolus autumnalis $\delta$ o fq, Triepeolus donatus $\delta$, pectoralis $\delta^{\gamma}+$, fq; Eucer.: Epimelissodes obliqua + , sc, Melissodes autumnalis $\hat{\sigma}$, coloradensis $\hat{\sigma}$, simillima $\hat{\sigma}$, fq, trinodis $\hat{\sigma} q$, se, fq.

Short-tongued Bees (8)-Halict.: Chloralictus pilosus + , Odontalictus ơ $q$, se, fq ; Collet.: Colletes compactus ơ $q$, se, fq ; Andren.: 
Pterandrena aliciae f. se, fq; Panurg.: Pseudopanurgus albitarsis

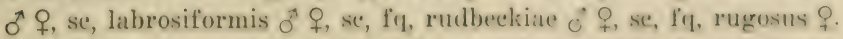

Other Hymenoptera (5) -Sphee.: Sphex gracilis fq, nigrieans. vulgaris: Bembic.: Bembix spinolate; Ścoli.: Scoulia bicincta.

Diptera (16)-Syrph.: Eristalis dimidiatus, transversus Syrphus ribesii f, n; Empid.: Parempis fq; Bombyli.: Anthrax alternata, haleyon, Exoprosopa decora, fasciata, Sparnopolius, Systoechus; Conop.: Zodion fulvifrons; Tuchin.: Arehytas aterrima. Cyphoeera, Peleteria fq. Senotainia trilineata, Spallanzania.

Lepidoptera (5)-Nymphal.: Charidryas, Vanessa huntera; Lycuen.: Cyaniris; Hesperi.: Limochores tammas; Ctenuch.: Serpsis.

Rudbeckia subtomentosa.-Blooms July 19-September 16; 97 visitors observed, July 30-September 2; Mis, Y.

Long-tongued Bees $(13)-A p .:$ Apis; Ceratin.: Ceratina f, se, ab; Megachil.: Megachile brevis of $q$, fq, petulans $q$, se, fq, 6-rlentatia q, Oligotropus $q$, Sayapis sayi $q$, se $;$ Epeol.: Epeolus bifasciatus of. Triepeolus eoncolor $q$; Eucer.: Epimelissodes illinoensis $q$, se.

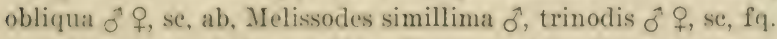

Short-tontrued Bees (1t)-Malict.: Chloralietus albipemis of,

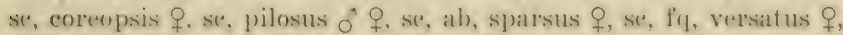
se, ab, zephyrus $q$, Curtisapis coriacea of, Evylaeus pectoralis of $q$, se, ab, IIalietus parallelus $\hat{\sigma}$, Odontalietus $\hat{\sigma} \hat{q}, \mathrm{se}, \mathrm{ab}$, Seladonia $\delta$ 을 se, fq; Panurg.: Pseudopanurgus albitarsis ${ }_{+}$, se, rudbeckiae $\delta$, se, fq, solidaginis $\delta^{\top} q$, se, ab.

Other Hymenoptera (16)-Sphec.: Priononyx atrata, thomae, Sphex gracilis, nigricans $\mathrm{fq}$, pietipennis $\mathrm{fq}$, vulgaris; Bembic.: Bembix nubillipennis, Bieyrtes ventralis ab, Stietiella; Scoli.: Elis 5-cineta, seolia bicincta fq; Eumen.: Leionotus anormis ab, arvensis fq, molestus, Pterochilus; Ichneumon.: Ceratosoma.

Diptera (35)-Syrph.: Eristalis latifrons fq, transwersus ab, Mesogramma geminata, murginata sf, ab, polita. Orthomeura nitiala, Sphaerophoria, Syritta ; Empid: Parempis ab; Bombyli.: Exopmsopa deeora, faseipennis fy. Lepidophora, Phthiria aldrichi, cinnta fq, Sparnopolius ab, Systoechus ab, Toxophnra; Conop.: Oncomyia, Zodion obliquefasciatum; Tachin: Cistogastrer oceidua fq. Eprigrimyia floridensis, Leuenstoma atra, siphonina, Oeyptera lata. tenuis, Peleteria, Phorantha humeralis, purpurasuens, Senotainia trilineata, Siphona, Siphoplaria anomala ab, Spallanzania ab: Musc.: Psembloyrelliat Agromyz.: Eusiphona ab; Oscin.: Siphonella cinerea.

Lepidoptera (12) - Nymphal.: Basilarchia archippus. Charidryas, Phyciodes ab; L.ycarn.: Chrysophamus, Cyaniris, Everes, 
Uranotes; Papilion.: Eurymus, Pontia ; Hesperi.: Hesperia, Limochores taumas; Ctenuch.: Scepsis.

Coleoptera (3)-Cerambyc.: Batyle f; Curculion.: Centrinus scutellum-album f; Lampyr.: Chauliognathus pennsylvanicus.

Hemiptera (4)—Caps.: Calocoris, Lygus ; Pentatom.: Euschistus ictericus; Beryt.: Harmostes.

The following 76 species and 669 individuals were taken on the flowers, July 30-August 27, 1909 :

Long-tongued Bees $(11: 44)-A p .:$ Apis 1 ; Ceratin.: Ceratina 11; Megachil: Megachile brevis 2, petulans 5, Oligotropus 1, Sayapis sayi 1; Epeol.: Epeolus bifasciatus 1; Triepeolus concolor 1; Eucer.: Epimelissodes illinoensis 1, obliqua 15, Melissodes trinodis 5 .

Short-tongued Bees (13:271)-Halict.: Chloralictus albipennis 1 , coreopsis 1 , pilosus 99 , sparsus 7 , versatus 56 , zephyrus 1, Curtisapis coriacea 1, Evylaeus pectoralis 28, Halictus parallelus 1, Odontalictus 69, Seladonia 5; Panurg.: Pseudopanurgus albitarsis 1 , solidaginis 1 .

Other Hymenoptera (12:59)—Sphec.: Priononyx atrata 2, Sphex gracilis 1, nigricans 6, pictipennis 1; Bembic.: Bembix nubillipennis 1, Bicyrtes ventralis 21, Stictiella 1; Scoli.: Elis 5cincta 3, Scolia bicincta 6 ; Eumen.: Leionotus anormis 9, arvensis 7 , molestus 1.

Diptera (28:247) -Syrph.: Eristalis latifrons 2, transversus 20, Mesogramma geminata 2, marginata 8, polita 2, Orthoneura nitida 2, Syritta 1; Empid.: Parempis 12; Bombyli.: Exoprosopa decora 1 , fascipennis 6 , Lepidophora 2, Phthiria aldrichi 5 , cincta 5, Sparnopolius 33, Systoechus 6, Toxophora 1; Conop.: Zodion obliquefasciatum 1; Tachin.: Cistogaster occidua 1, Epigrimyia floridensis 1, Ocyptera tenuis 5, Peleteria 2, Phorantha humeralis 1, Senotainia trilineata 2, Siphona 1, Siphoplagia anomala 49, Spallanzania 57; Musc.: Pseudopyrellia 1; Agromyz.: Eusiphona 18.

Lepidoptera (9:41)-Nymphal.: Basilarchia archippus 2, Charidryas 1, Phyciodes 32; Lycaen.: Chrysophanus 1, Everes 1; Papilion.: Eurymus 1, Pontia 1; Hesperi.: Limochores taumas 1; Ctenuch.: Scepsis 1.

Coleoptera $(3: 7)$-Cerambyc.: Batyle 2 ; Curculion.: Centrinus scutellum-album 3; Lampyr.: Chauliognathus pennsylvanicus 2.

Rudbeckia triloba (15:467-8).-Blooms July 19-October 26; 107 visitors observed, July 23-October 1; Mas, Y.

Long-tongued Bees (23)-Ap.: Apis se; Bomb.: Bombias auri-

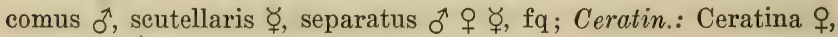
sc, ab; Stelid.: Stelidium ô; Megachil.: Trypetini: Neotrypetes 


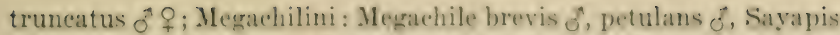
sayi + , se; Coelioxyini : Coelioxys alternata of of s-dentata $q$, sayi o; Epeol. Triepeolus concolor \&, eressunii of of. fq, helianthi of; Eucer.: Epimelisodes obliqua of of, se, ah, Melissodes agilis of $q$, fq, bimaculata of $q$, boltmiae $q$, e, simillima $f$, trinodis $f ?$, se, $\mathrm{fq}$, vernoniana $\delta$ f.

Short-tongued Bees (25)-IIalict.: Agapostemon radiatus d.

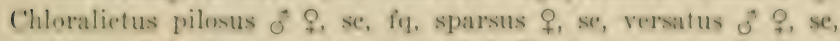
aphyrus of $f$. Curtisapis coriacea of $f$. Lyylueus pectoralis ?. se. ab, Halictus lerouxii of, Odontalictus of $f$, se, ab, Oxystoglassa confusa $q$, se, pura $q$, se, similis $q$, se, Seladonia of of Collet.: Colletes amerieanus $q$, se, eompaetus $q$, se; Andren.: Pterandrena alieiae

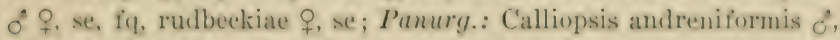
coloradensis of $\%$, se, Pseudopanurgus asteris $q$, se, compositarum $d$. labrosiformis $q$, se, labrosus of $q$, se, $a b$, rudbeckiae of $q$, se, $a b$, in cop, type, rugosus $\delta^{\top}+$, se.

Other Hymenoptera (12) - Sphec.: Sphex gracilis, nigrieans; Bembic.: Bembix spinolae, Bieyrtes ventralis, Stietiella; Scoli.: Elis interrupta, 5-cincta, Scolia bicincta ; Vesp.: Polistes variatus; Eumen.: Leionotus arvensis; Oxybel.: Notoglossal americana; Ichneumon.: Exetastes suaveolens.

Diptera (37)-Syrph.: Eristalis latifrons, transversus fy, Mallota cimbiciformis, Spilomyia longieornis, Volucella fasciata; Empid.: Parempis $\mathrm{fq}$; Bombyli.: Anthrax fulvohirta, haleyon $\mathrm{fq}$, Argyramoeba oedipus $f, n$, Exoprosopa decora, fasciata, faseipennis, Geron rufipes, Lepidophora, Phthiria cincta, Sparnopolius fq. Systoechus, Systropus, Toxophora; Conop.: Conops brachyrrhynchus. Oneomyia, Zodion obliquefaseiatum; Tachin.: Apinops, Arehytas analis, Cistogaster oecidua, Epigrimyia geniculata ab, illinoensis type, Leskiomima secunda, Ocyptera temuis, Peleteria fy, Senotainia trilineata, Siphoplagia anomala ab, Spallanzania, Triohophora; Dexi.: Ptilodexia; Muse.: Pseudopyrellia; Agromyz.: Eusiphona.

Lopidoptera (7)-Nymphal.: Basilarehia arehippus, Charidryas fq. Phyciodes fq: Lycaen.: Everes; Papilion.: Eurymus, Pontia : Hesperi.: Polites.

Coleoptera (3)-Lumpyr.: Chaulioguathus penusylvanieus ab; Molo.: Epicauta pennsylvanica f, n; Mordell.: Mordalla marginata $f, n$.

The followine 23 visitors and $1: 39$ individuals were talien on the flowers on August 20, 24:

Long-tongued bees $(5: 7)-B o m b$.: Bombias anrieomus 1: Crutin.: Ceratina 1; Meguichil: Mrgachile petulans 1; Eucer: Epimelissodes obliqua 2, Melissodes boltoniae 2. 
Short-tongued Bees (6:44)-Halict.: Chloralictus pilosus 2, versatus 1, Evylaeus pectoralis 2, Odontalictus 36, Oxystoglossa similis 1; Panurg.: Pseudopanurgus rudbeckiae 2.

Other Hymenoptera (2:3)—Sphec.: Sphex nigricans 1; Eumen.: Leionotus arvensis 2.

Diptera (6:34)-Syrph.: Eristalis transversus 6; Bombyli.: Sparnopolius 3, Systoechus 1; Tachin.: Cistogaster occidua 1, Siphoplagia anomala 22; Musc.: Pseudopyrellia 1.

Lepidoptera (2:7)-Nymphal.: Basilarchia archippus 1, Phyciodes 6.

Coleoptera (2:43)-Lampyr.: Chauliognathus pennsylvanicus 42 ; Epicauta pennsylvanica 1.

Senecio aureus.-Blooms May 7-June 5; 18 visitors observed on May 15, June 2 ; Mis, Y.

Long-tongued Bees (4)-Ceratin.: Ceratina ô; Nomad.: Centrias americanus $\delta^{\top}$, Holonomada superba $\delta \uparrow$, fq; Eucer.: Tetralonia dilecta 0 , fq.

Short-tongued Bees (5, ९)-Halict.: Chloralictus pilosus se, ab, pruinosus se, Odontalictus se, ab, Oxystoglossa confusa, se, similis.

Other Hymenoptera (1)-Eumen.: Leionotus clypeatus.

Diptera (8)—Syrph.: Eristalis latifrons, Mesogramma marginata, Sphaerophoria, Tropidia mamillata; Conop.: Zodion fulvifrons; Tachin.: Cistogaster occidua, Epigrimyia floridensis fq, Peleteria.

Silphium integrifolium (15: 463).--Blooms June 10-September $20 ; 24$ visitors observed, July 7-September 16; Mas, Y.

Long-tongued Bees (18)-Ap.: Apis se; Bomb.: Bombus con-

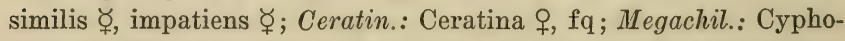
pyga $\delta^{\lambda}$, Megachile brevis $\sigma^{\lambda}$, mendica $q$, petulans $\delta$, Sayapis pol-

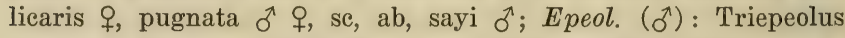
concavus, remigatus, simplex; Eucer.: Epimelissodes obliqua $\delta$, Melissodes agilis $\sigma^{\lambda}, \mathrm{fq}$, coloradensis $\sigma^{\lambda} q$, se, trinodis $\sigma^{\lambda}$, fq.

Short-tongued Bees (3)-Halict.: Agapostemon radiatus $\delta$, se, Odontalictus $q$; Andren.: Pterandrena pulchella $\$$.

Diptera (3)-Bombyli.: Systoechus; Conop.: Physocephala tibialis; Tachin.: Cistogaster occidua.

Silphium laciniatum $(15: 463-4)$.-Blooms June 21-August 30 ; 29 visitors observed, July 16 -August 6 ; Mas, Y.

Long-tongued Bees (13)-Ap.: Apis; Bomb.: Bombias separatus

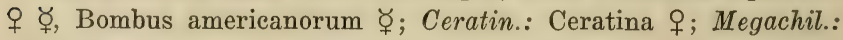
(o) : Megachile brevis, 6-dentata, Sayapis pugnata, sc; Epeol.: Tri- 
epeolus eoucavus of, concolor of $q$; Eucer.: Epinelissodes ubliqua of, Melissodes agrilis $\vec{\delta} q$, ab, bimaculata $\vec{\sigma}$, coloradensis of $f, s c$, lip.

Short-tongued Bees $(5,7)$ - Maliel.: Arapostemon texamus se, viridulus se, Chloralietus pilosus e, n, sparsus e, n, Odontalietus c, $x$.

Diptera (9)-Syrph.: Illorrapta, Eristalis latifroms, transwersus, Tropidia mamillata; Bombyli.: Anthrax altermata f, n, Geron rufipes, Systuechus; Conup.: Zudion fulvifrons, obliquefa-ciatum.

Lepieloptera (2) - Nymphal.: Anosia : Paprition.: Eur!mus iq.

Silphium perfoliatum (16:176-7).-Blooms July 9-September 12; 75 visiturs observed, July 2:3-septemher 12; Mas, Y.

Long-tongued Bees $(26)-A p$.: Apis se, fif Bomb.: Bombias

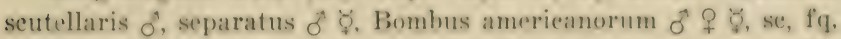
eonsimilis $\varnothing$, se, fq, impatiens $\not$, se, fq ; Cratin.: Ceratina $q$, se, fq; Meyachil.: Megachilini: Megachile brevis of $q$, se, mendica $\delta^{2}$ q, se,

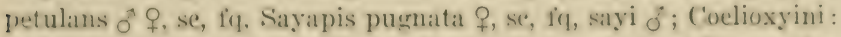
Coelioxy sermana of; Eivol.: Triepeolus eonearus of f. eoneolor

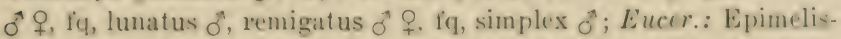

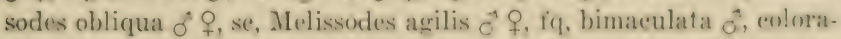

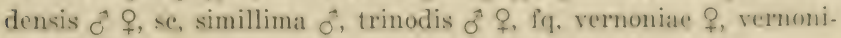
ana $\delta$, fq.

Short-tongued Bees (14) - Malict.: Agapostemon radiatus of f.

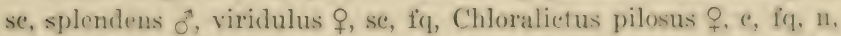
sparsus $q, e, f q, n$, versatus $\%, e, n$, Evylaeus pectoralis $q, e, n$, Halietus lerouxii $q$, Odontalietus $q$, se, Oxystoglossa cónfusa $q ; A n$ -

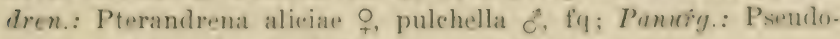
panurgus labrosiformis $\bar{\sigma}, \mathrm{fq}$, rugosus $\delta^{\Uparrow}$ ㅇ.

Other IIymenoptera (3)-Sphrc.: Sphex procera; Sculi.: Seolia bicineta; Eumen. : Leionotus dorsalis.

Diptera (12) Siyruh.: Mllograpta f, n, Eristalis tenax, transverous. Milodia i, n ; Bombyli.: Anthrax alternata, i. n, halryon, Exoprosopa fasciata, Geron rufipes, Sparmopmlius, Systoechus fy; Conop.: Zodion obliquefasciatum; Tachin.: Arehytas aterrima.

Lepidoptera (20)-Xymphal.: Anosia, Bavilarehia archippus, astyanax, Charidryas, Polyeonia interrogationis, Vanessa atalanta. eardui, huntera; Lycaen.: Chrysophanus; Papilion.: Euphomales. Eurymus, Heraclides, Jasouiades, Laertias, Pontia ; Iresperi: Atrytone delaware, zabulon, Epareyreus, Limochores taumas, Pholiona eatullus.

Silphium terebinthinaceum.-Bloums July 2-September 80 : $1:$ : visitors observed, August 9-28; Mas, X. 
Birds (1)-Trochil.: Trochilus.

Long-tongued Bees (7)-Ap.: Apis se, fq; Bomb.: Bombus americanorum $\Varangle$, impatiens $\not$, se; Ceratin.: Ceratina $q$, se; Megachil.: Megachile brevis + ; Eucer.: Epimelissodes obliqua + , se, Melissodes coloradensis $0^{\top}$.

Short-tongued Bees (2)-Halict.: Agapostemon radiatus $\hat{\delta}$, Odontalictus $0^{\wedge}$ o, se, fq.

Other Hymenoptera (1) -Sphec.: Sphex procera.

Diptera (2)-Bombyli.: Sparnopolius, Systoechus.

Solidago canadensis $(15: 455-6)$.-Blooms August 1-October 17 ; 218 visitors observed, August 1-October 10; Mis, Y.

Long-tongued Bees (22)-Ap.: Apis se, ab; Bomb.: Bombias

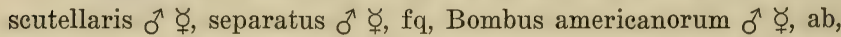
consimilis $\delta$, impatiens $\hat{\sigma} q \not{q}, \mathrm{se}, \mathrm{fq}$, Psithyrus variabilis $q$; Ceratin.: Ceratina $q$, sc; Stelid.: Stelidium o $q$, fq; Megachil.: Trypetini: Neotrypetes productus $\delta^{\pi}$, truncatus $\delta^{\pi}+$, sc, ab; Osmiini : Ashmeadiella $q$, se ; Coelioxyini : Coelioxys sayi $\sigma^{\top}+$; Nomad.: Holonomada placida $\delta^{\Uparrow}$, Nomada vicina $\delta^{\Uparrow}$; Epeol.: Epeolus autumnalis + , pusillus $\delta^{\lambda}+$, Triepeolus pectoralis $\delta^{\lambda}$; Eucer.: Melissodes autumnalis $q$, se, boltoniae $q$, se, nivea $q$, se, simillima $\delta$.

Short-tongued Bees (28)-Halict.: Augochlora viridula $\sigma^{\lambda}$, Chloralictus albipennis $q$, se, versatus $\delta^{\lambda}$, ab, Curtisapis coriacea $q$, se, forbesii $0^{\top} q$, se, Halictus lerouxii $q$, se, Odontalictus $q$, se, Seladonia $\delta$ 옹 sc; Sphecodini: Machaeris stygia $\delta$, Sphecodes heraclei $q$, Sphecodium cressonii $\delta^{\lambda}+$, fq, smilacinae $\delta^{\lambda}$; Prosopid. $\left(\delta^{\lambda}+\right)$ : Prosopis illinoensis fq, pygmaea ab, sayi ab, ziziae sf, $a b$; Collet.: Colletes americanus $\delta^{\lambda}+\mathrm{se}, \mathrm{ab}$, armatus $\delta^{\lambda}+\mathrm{se}, \mathrm{fq}$, compactus + , sc, eulophi $\delta^{\lambda}$; Andren. ( $\left.\delta^{\lambda}+, \mathrm{se}, \mathrm{ab}\right)$ : Andrena nubecula, Pterandrena solidaginis in cop, type; Panurg.: Calliopsis coloradensis $\sigma^{\lambda}+, \mathrm{sc}, \mathrm{fq}$, in cop, Heterosarus $q$, se, Perdita $\sigma^{\lambda}+, \mathrm{sc}, \mathrm{fq}$, in cop, Pseudopanurgus asteris $\hat{\sigma}+$, se, fq, in cop, labrosiformis $\hat{\partial}$, solidaginis $\sigma^{\lambda}+$, se, ab, type.

Other Hymenoptera (81)—Sphec.: Ammobia ichneumonea, pennsylvanica, Isodontia apicalis, Priononyx atrata, thomae, Sceliphron, Sphex vulgaris; Bembic.: Stizus; Scoli.: Elis interrupta, 5-cincta, Scolia bicincta, nobilitata; Philanth.: Cerceris compacta, finitima, fulvipes fq, kennicottii, prominens, Eucerceris fq; Vesp.: Polistes annularis ab, pallipes ab, rubiginosus, variatus ab, Vespula cuneata, germanica; Eumen.: Ancistrocerus campestris, capra ab, eatskillensis, tigris ab, Eumenes fraternus, Leionotus anormis, arvensis, foraminatus, scrophulariae fq, ziziae, Zethus; Crabron.: Anacrabro fq, Clytochrysus, Hypocrabro, Lindenius errans, Lopho- 
crabro, Solenius, Synothyreupus, Nestorrabro 6-maculatus; Oxybel.: Notoglossa amerieana $f q$, frontalis fi, Oxybelus niger, 4-notatus; Pemphredon.: Mimesa; Larr.: Larropsis iq. Tachyspluex acutus; Nyssmo: Pseudoplisus phaleratus; Pompil.: Anoplius illinoensis type, Ceropales bipunctata, elegans, fraterna, iulvipes, Cryptocheilus agenioides type, Episyron fy, Poecilopompilus interruptus, navus, P’ompiloides marginatus, subviolaceus, Prioenemoides fulvicornis, Psammochares relativus; Tiphi.: Tiplia punetata ; Chrysid.: Hedyehrum violaceum ; Chalcid.: Leueospis iq, Perilampus fulvieornis, robertsonii; Euani.: Foenus tarsatorius; Ichneumon.: Ceratosomat, Cremastus retiniae, Lampronota americana, varia, Limneria eurycreontis Metopius polycinctorius; Brucon.: Agathis areolata fi, type, Bracon simplex, vernoniae, chelonus, Iphiaulax ab.

Diptera (62)-Stratiomy.: Nimotelus; Syrph.: Allograpta, Chilosia punetulata, Eristalis bastardi, dimidiatus ab, flavipes, latifrons, tenax, Helophilus latifrons, similis sf, ab, Mesogramma geminata, polita fq, Milesia, Orthoneura nitida si, Paragus bicolor, Platychirus hyperboreus, quadratus, Spilomyia longieornis, quadriiasciata, Syritta, Syrphus americanus, Tropidia mamillata f Xanthorramma emarginata; Empid.: Parempis; Bombyli.: Lxuprosopa decora, fuscipennis, Geron rufipes, Sparnopolius, Toxophora; Conop.: Conops brachyrrhynchus, sylvosus, xanthopareus, Oneomyia, Physoecphala texana, tibialis, Robertsonomyia ; Tachin.: Actia, Amobia distincta type, Apinops, Belvosia unifaseiata, Cistogaster occidua, Euryceromyia robertsonii typc, Grmnosoma, Ililarella aristalis type, Ennyomma clistoides type, nigrifrons type, Phasioclista metalliea type, Ocyptera tenuis, Pachyophthalmus sienatus, Senotainia trilineata, Triehopola plumipes; Sarcopha! .: IIelicobia helicis, Sareophaga cimbicis, hunteri; Musc.: Compsomyia fq, Graphomyia, Morellia, Pseudopyrellia ab, Stomoxys; Anthimm.: Calythea, Phorbia aera; Trypet.: Trypeta humilis.

Lepidoptera (8)-Nymphal.: Anosia, Vanessal rardui; Lyouen.: Chrysophanus; Pupition.: Eurymus ; Ctenuch.: Ścepsis: Nuclu. Carneades, Feltia, Heliothis armiger ab.

Coleoptera (14)-Bruch.: Bruchus obsoletus; Carab.: Callica i: Cerambyce: Cyllene decorus i, robiniae i, fq; Chrysumel. (1): D) iabrotira 12-punetata ab, longieurnis ab, vittata: Corcinell.: Cnecinclla 9-notata, Hippodamea glarialis fq. Megilla f; Lampyr.: Chauliognathus pennsylvanicus ab; Moloch.: Collops: Melo.: Epicauta penusylvaniea $f$, ab; Scarabae.: Euphoria sepulehralis $f$.

Hemiptera (3)-Caps.: Calocoris: Core.: Alydus pilonulus; Lygac.: Lygacus. 
Solidago graminifolia (15: 457-8).-Blooms July 29-September 29 ; 44 visitors observed, September $4-25$; Mis, Y.

Long-tongued Bees (7)-Ap.: Apis sc, ab; Bomb.: Bombias scutellaris $\not{q}$, separatus $\hat{\sigma}$, Bombus americanorum $\hat{\sigma} \hat{\psi}, \mathrm{fq}$, impatiens $\Varangle$, se, fq; Eucer.: Melissodes boltoniae $q$ sc, nivea $q$, se, type.

Short-tongued Bees (4, $q$, se)-Halict.: Chloralictus versatus, Odontalictus; Collet.: Colletes armatus; Andren.: Pterandrena solidaginis.

Other Hymenoptera (14)—Sphec.: Priononyx atrata; Bembic.: Bembix nubillipennis, Megastizus; Scoli.: Elis 5-cincta, Scolia bieincta; Philanth.: Eucerceris; Vesp.: Polistes pallipes, variatus; Eumen.: Ancistrocerus capra ab, eatskillensis, tigris; Crabron.: Hypocrabro, Xestocrabo 6-maculatus; Larr.: Larropsis; Pompil.: Priocnemoides fulvicornis.

Diptera (9)—Syrph.: Helophilus latifrons, similis ab, Sphaerophoria, Spilomyia 4-fasciata; Tachin.: Cistogaster occidua, Paradidyma, Senotainia rubriventris; Musc.: Compsomyia, Pseudopyrellia.

Lepidoptera (8)-Nymphal.: Phyciodes, Vanessa huntera; Lycaen.: Chrysophanus, Everes; Papilion.: Eurymus; Arcti.: Utetheisa; Cramb.: Crambus; Noctu.: Heliothis armiger.

Coleoptera (2)-Lampyr.: Chauliognathus pennsylvanicus ab; Melo.: Epicauta pennsylvanica ab.

Solidago missouriensis (15:454-5).-Blooms June 26-August 22 ; 69 visitors observed, July 21-August 19; Mis, Y.

Long-tongued Bees (6)-Ap.: Apis; Ceratin.: Ceratina ô; Megachil.: Coelioxys 8-dentata $q$; Epeol.: Epeolus bifasciatus $q$; Eucer.: Epimelissodes obliqua + , se, fq, Melissodes boltoniae + , se.

Short-tongued Bees (10)-Halict.: Chloralictus albipennis + , se, sparsus $q$, se, tegularis $q$, se, versatus $\sigma^{1}+$, se, ab, Evylaeus pectoralis $\sigma^{\top} q$, se, Odontalictus $q$, se, ab, Oxystoglossa similis $q$, Seladonia ; Sphecodini: Machaeris stygia $q$; Prosopid.: Prosopis pygmaea ot 9 , ab.

Other Hymenoptera (31)—Sphec.: Ammobia ichneumonea, pennsylvanica, Priononyx atrata, Sphex nigricans, pictipennis, vulgaris; Scoli: : Elis interrupta, 5-cincta ab, Scolia bicincta fq; Philanth.: Cerceris clypeata, compacta, finitima, Eucerceris, Philanthus ventilabris; Vesp. Polistes variatus; Eumen.: Eumenes fraternus, Leionotus anormis, arvensis fq, histrionalis, vagus; Crabron.: Protothyreopus bigeminus, Solenius; Oxybel.: Notoglossa americana, frontalis, Oxybelus 4-notatus; Larr.: Larropsis fq ; Nysson.: Silaon; 
Chrysid.: Hedychrum wiltii ab; Chalcil.: Perilampus ryaneus; Ichneumon.: Ceratosoma fq; Bracon.: Mierodus.

Diftera (17)-syrph.: Mesogramma marginata, Syritta; Lmpid.: Parempis; Bombyli.: Exoprosopa faseipenuis fq; Conop.: Yodion obliquefaseiatum; Turhin.: Alophora aenooventris, Cistogaster aecidua, Gymmoma, Gymnoproopa, Pliorantha magna, pruinosa, Senotainia rubriventris, trilimeata fy. Siphoplagia anom-

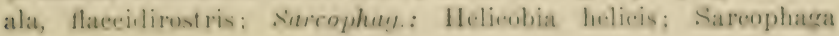
hunteri.

Colcoptera (4) Chrysomel.: Diabrotica 12-punetata 1; Lumpyr.: Chaulinguathus junusylvanieus: Melu.: Ejieauta grenusylranica $\mathrm{f}$, ab, n; lihipiphor.: Myodites.

Hemiptera (1)-Lygate. Lygacus.

July 30,1909 , the following 13 speries and :3: individuals were taken on the flowers:

Lone-tongued Beos (1:1)-Mrgachil.: Coelioxys S-dentata 1.

Shert-tongued Bees (3: 19)-IIalict.: Chloralictus sprarsus 4 , versatus 10; Prosopid.: Prosopis pygmaea 5.

Other Hymenoptera $(5: 5)$ - Sphec.: Sphex vulearis 1 ; Philanth.: Cereeris finitima 1 ; Eumen.: Leionofus histrionalis 1 ; Oxybel.: Notoglossa amerieana $1 ;$ Nysson.: Silaon 1.

Diptera (4:7)-iyrph: Mesogramma marginata 1, Syritta 3; Empid.: Parempis 1; Tachin.: Cistogaster occidua 2.

Solidago nemoralis $(15: 457)$.- Blooms September 9-0)tober 1) : 92 visitors observed. September 19-October 9; Mis, Y.

Long-fungued Bees ( 8$)-A p$.: Apis se, ah; Bomb.: Bombias aurienmus of. Bombus americanorum of $q \delta$. impatiens $\delta$; Ceratin.:

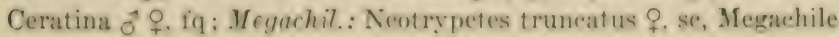
brevis $q$, se; Epeol.: Triepeolus cressonii $q$.

Short-tongued Bees (15)-IIuliel.: Chloralietus illinoensis o, verwatus $\mathcal{C}$ P. se, fq. Evylaeus pectoralis $q$, Odontalictus d, Oxystoeloses similis 9. Paralictus platyparius $9 ;$ P'rosopid.: Prosopis

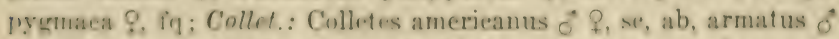
?. se, fy, compatetus P. se. fq. euloplii o. se; Andren.: Andrena nubecula $\&$. s. ah. Pterandrena asteris \&, se: Ponurg.: P'smulopanurgus asteris $q$, se, compositarum $q$.

Other Hymenoptera (24)_Sphe. Splex protipenuis, procera, vulquris: Philanth.: Cereeris clypeata, finitima, kennicottii. Philanthus punetatus: Vesp.: P'olistes anmularis ab, pallipes fy. rubiginosus, variatus fq; Eumen.: Ineistroenrus catskillensis, tigris, J.eionotus anornis, Poraminatus, histrioualis : Crabron.: Prototbyroopus rufifemur. Solenins fq: Orybel.: Notrequesa Prontalis: Pomphre. 
don.: Mimesa; Larr.: Larropsis; Pompil.: Anoplius illinoensis, Ceropales fraterna, fulvipes, Planiceps niger, Pompiloides marginatus; Chrysid.: Gonochrysis; Bracon.: Chelonus.

Diptera (27)-Syrph.: Mesogramma geminata, Sphaerophoria, Spilomyia longicornis, Syritta sf, Syrphus americanus sf, arcuatus; Bombyli.: Sparnopolius, Systropus; Conop.: Oncomyia; Tachin.: Archytas aterrima, Gymnosoma, Pachyophthalmus signatus fq, Polidea, Trichopoda pennipes, Xanthomelana; Sarcophag.: Helicobia helicis, Sarcophaga cimbicis, hunteri; Musc.: Compsomyia, Lucilia caesar, sericata, Musca, Pseudopyrellia, Stomoxys; Oscin.: Chlorops proxima; Trypet.: Trypeta humilis, solaris.

Lepidoptera (6)-Nymphal.: Anosia; Papilion.: Eurema, Eurymus; Arcti.: Utetheisa; Ctenuch.: Scepsis; Noctu.: Heliothis armiger.

Coleoptera (5)-Cerambyc.: Cyllene robiniae f; Chrysomel.: Diabrotica longicornis ; Coccinell.: Coceinella 9-notata f; Lampyr.: Chauliognathus pennsylvanicus; Melo.: Epicauta pennsylvanica f, $\mathrm{ab}$, in cop.

Hemiptera (3)-Caps.: Lygus; Core.: Alydus pilosulus; Lygae.: Lygaeus.

October 4, 1909, the following 17 species and 56 individuals were taken on the flowers:

Long-tongued Bees (1:21)-Ap.: Apis 21.

Short-tongued Bees (2: 10)-Andren.: Andrena nubecula 9; Panurg.: Pseudopanurgus asteris 1.

Other Hymenoptera (4:12)-Vesp.: Polistes annularis 9, variatus 1; Eumen.: Ancistrocerus catskillensis 1, tigris 1.

Diptera (5: 5)—Syrph.: Mesogramma geminata 1; Bombyli.: Sparnopolius 1; Tachin.: Xanthomelana 1; Musc.: Lucilia caesar 1, Pseudopyrellia 1.

Lepidoptera (1:1)-Ctenuch.: Scepsis 1.

Coleoptera (3:6)-Cerambyc.: Cyllene robiniae 1; Chrysomel.: Diabrotica longicornis 3 ; Lampyr.: Chauliognathus pennsylvanicus 2.

Hemiptera (1: 1)-Lygae.: Lygaeus 1.

Solidago rigida.-Blooms August 23-October 7; 52 visitors observed, September 4-25; Mas, Y.

Long-tongued Bees (9)-Ap.: Apis; Bomb.: Bombus ameri-

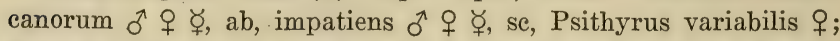
Megachil.: Megachile brevis $q$, se, Xanthosarus $q$; Eucer.: Epimelissodes obliqua $q$, se, Melissodes autumnalis $0^{\lambda} q, \mathrm{se}$, ab, nivea $q$. 
Short-tongued Bees (3)-Malict.: Chloralietus versatus o : Collet.: Colletes amerieanus $q$, se, compactus $\delta$.

Other Hymenoptera (16)-Sphec: Priouonyx atrata, thomae, Sphex procera; Philenth.: Cereeris compacta, prominens; Tesp.: Polistes annularis, pallipes, variatus; Eumen.: Ancistroeerus eapra, tigris fq. Leionotus arvensis, foraminatus, serophulariae; Crabron.: Protothyreopus rufifemur, Solenius; P'umpil.: Ageniella pet iolata.

Diptera (13)-Syrph.: Paragus bicolor, tibialis $\mathrm{f}$. n. Sphacrophoria, Syritta; Bombyli.: Spamopolius ah; Conop.: Stylogaster biamulata: Tachin. Cistogaster oceidua, Linnacmyia, Pachy. ophthalmus signatus, Spallanzania: Sarcophag.: Sarcophaga cimbicis; Musc.: Compsomyia, Pseudopyrellia.

Lepidoptera (7) - Nymphal.: Anosia, Basilarchia arehippus; Lycaen.: Everes, Heodes; Papition.: Eurymus; Hesperi.: Polites; Noctu.: Drasteria erechtea.

Coleoptera (4)-Chrysomrl. (f, n): Diabrotica 12-punctata, longreornis ab; Lampyr.: Chauliognathus pennsylvanicus ab; Melo.: Epicauta pennsylvanica $\mathrm{f}, \mathrm{ab}, \mathrm{n}$.

September $18,25,1909$, the following 29 species and 146 individuals were taken on the flowers:

Long-tongued Bees $(6: 51)-A p .:$ Apis 2; Bomb.: Bombus amerieanorum 35, impatiens 3, Psithyrus variabilis 1; Megachil.: Megachile brevis 1 ; Eucer.: Melissodes autumnalis 9.

Short-tongued Bees (2:2)-Collct.: Colletes americanus 1, compactus 1.

Other Iymenoptera $(9: 17)--$ Sphuc.: Sphex proeera 2 ; Philanth.: Cerceris compacta 1; Vcsp.: Polistes annularis 1, frallipes 1, variatus 2 ; Eumen.: Ancistrocerus capra 2, tigris 5, Leionotus foraminatus 2, serophulariae 1.

Diptera $(5: 22)$-Syrph.: Paragus tibialis 1, Sphaerophoria 2; Bombyli: Sparnopolius 17 ; T'achin.: Spallanzania 1; Musc.: Compsomyia 1.

Leppidoptera (3:4)-Lycuen.: Everes 1, Heodes 1; Voctu.: Drasteria erechtea 2.

Coleofitera $(4: 50 ; 3: 25 \mathrm{n})$-C'hrysomel.: Diabrotica 12-punetata 1. longicornis 11 ; Lampyr.: Chauliognathus pennsylvanieus 25; Melo.: Epicauta pennsylvanica 13, n.

Solidago serotina.-Blooms Aurust 24-Siptember 27: seven visiturs obersel. Auguet 30-September 20; Mas (Mis). Y.

Long-tongued Bees $(3)-$ Bomb.: Bumbus americanorum $\phi$. consimilis $\Varangle ;$ Eucer.: Melissodes simillima $\delta$.

Short-tongued Bes (1) - Panurg, : Perulopanurgus asteris a, se. 
Other Hymenoptera (1)-Crabron.: Xestocrabro 6-maculatus.

Diptera (1)-Syrph.: Spilomyia 4-fasciata.

Coleoptera (1) -Cerambyc.: Cyllene robiniae $\mathrm{f}, \mathrm{n}$.

Solidago speciosa.-Blooms September 12-October 17; nine visitors observed, September 23-October 6; Mas, Y.

Long-tongued Bees (5)-Ap.: Apis fq; Bomb.: Bombias auricomus $q$, Bombus americanorum $\delta \bar{\gamma}$, fq, impatiens $\delta^{\lambda}+$, Psithyrus variabilis .

Other Hymenoptera (1)-Vesp.: Polistes variatus.

Lepidoptera (2)-Nymphal.: Vanessa huntera; Ctenuch.: Scepsis fq.

Coleoptera (1) -Lampyr.: Chauliognathus pennsylvanicus fq.

Solidago ulmifolia.-Blooms August 20-October 7; 52 visitors observed, August 22-September 20; Mis, Y.

Long-tongued Bees (7)-Bomb.: Bombus impatiens $\delta^{7}$; Megachil.: Neotrypetes truncatus $\delta^{\lambda}+$, se, fq; Megachile brevis $\sigma^{\lambda}$; Eucer. ( $($, sc) : Melissodes autumnalis, boltoniae, simillima, trinodis.

Short-tongued Bees (15)-Halict.: Chloralictus sparsus $\delta^{7}$, se, Evylaeus pectoralis + , se; Sphecodini: Sphecodium eressonii $\widehat{\delta}+$; Prosopid.: Prosopis illinoensis $q$, pygmaea $q$, ziziae $\delta^{\lambda} q$; Collet.: Colletes americanus $q$, sc, armatus $\delta \circ$, se; Andren.: Andrena nubecula $\sigma^{\lambda}+$, se, fq, Pterandrena asteris $\delta^{\lambda}+$, se, solidaginis $q$, sc, fq; Panurg. (se) : Heterosarus $q$, Perdita $q$, Pseudopanurgus asteris $\delta q, a b$, compositarum $q$.

Other Hymenoptera (14)-Spliec.: Sphex vulgaris; Philanth.: Cerceris astarte, prominens; Vesp.: Polistes annularis, pallipes; Eumen.: Ancistrocerus campestris, tigris, Eumenes fraternus, Leionotus scrophulariae; Crabron.: Rhopalum scutellatum, Synothyreopus; Larr.: Larropsis; Chrysid.: Hedychrum violaceum; Nysson.: Alyson.

Diptera (15)-Syrph.: Allograpta, Helophilus similis, Spilomyia longicornis, Syritta, Syrphus americanus; Empid.: Parempis; Bombyli.: Anthrax alternata f, Exoprosopa decora, Geron rufipes, Sparnopolius; Tachin.: Archytas aterrima, Ennyomma nigrifrons, Peleteria, Siphona; Musc.: Morellia.

Hemiptera (1)-Caps.: Lygus.

Taraxacum officinale (int.).-Blooms March 23-November 27; 18 visitors observed, March 28-May 28; Mis, Y.

Long-tongued Bees (5)-Ap.: Apis se, fq; Bomb.: Bombus impatiens + ; Megachil.: Ceratosmia $\delta^{\lambda}$, Osmia purnila $\delta^{\lambda}$; Nomad.: Nomada denticulata $\sigma^{\top}$. 
Short-tongued Bees (6)-Uulict. ( $९)$ : Agapostemon radiatus se, Chloralietus versatus fq, Odontalietus se; Andren. $\left(\sigma^{*}\right)$ : Andrena dunningii, Opandrena eressonii, miserabilis fq.

Diptera (6) -Syrph.: Eristalis aeneus; Bombyli.: Bombylius major, pulchellus; Conop.: Myopa vesiculosia Musc: Pseudopyrellia; Anthomy.: Phorbia acra.

Lepidoptera (1)-Nymphal.: Vanessa huntera.

Verbesina helianthoides $(15: 471-2)$.-Blooms June 6-Aurust $10 ; 46$ visitors observed, June 10-July 11; Mas, Y.

Lung-tongued Bees (21)-Ap.: Apis se, fq ; Bomb.: Bombias aurieomus $q$, Bombus americanorum o $\not$; Ceratin.: Ceratina $q$, iq; Megachil.: Trypetini: Neotrypetes truncatus o, se; Osmiini : Ashmeadiclla $q$, se, Hoplitis $q$; Megachilini: Cyphopyga of $q$,

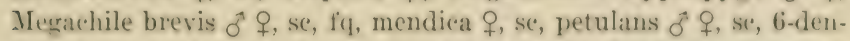
tata $q$, Sayapis polliearis $\delta$, pugnata of $q, \mathrm{se}, \mathrm{fq}$, Xanthosarus of; Coelioxyini: Coelioxys alternata of, 8-dentata of, rufitarsis of; Epeol.: Epeolus bifaseiatus of; Eucer.: Epimelissodes obliqua o, Tetralonia dilecta ㅇ.

Short-tongued Bees (14)_Ilalicl.: Agapostemon viridulus \&, Chloralietus pilosus $q$, se, sparsus $q$, e, n, versatus $q$, se, Curtisap is eoriacea $q$, Evylacus areuafus $q$, Halictus lerouxii $q$, se, Odontalietus of $q$, se. Oxystoglossa pura of, similis $q$, se: Collet.: Colletes eulophi $\$$, se ; Andren.: Pterandrena rudbeckiae $\$$; Panurg.: Calliopsis andreniformis $q$, se, Pseudopanurgus albitarsis of $q$. se.

Other Hymenoptera (4)_Sphec. Sphex nigrieans if, pietipennis, procera fq; Bembic.: Stictiella.

Diptera (4)-Syrph.: Eristalis transversus, Mesngramma marginata $f, n$; Bomloyli.: Anthrax parvienrnis, Systoechus.

Lepidoptera (3)-Nymphal.: Phyeiodes; Hesperi: Thanaos juvenalis; Ctenuch.: Seepsis.

Vernonia fasciculata $(15: 451-2)$.- Blooms July 1-0rtwher 1; 45 visitors observed, July 21-September 24; Mas, R.

Lme-tongued Bers (20)-.1p.: Apis; Bomb.: Bombias auri-

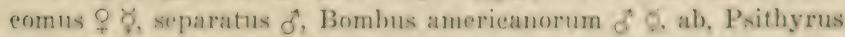
variabilis o \&, fiq: Crratin.: Ceratina \&. se; Megackil.: Neotrypetes tmuneatus f, se; Sayapis sayi of $q$. Xanthosarus $d$ f, se; Eprol.:

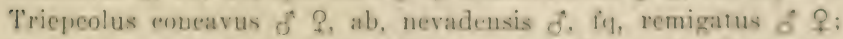
Eucr.: Epimelissodes atripes f, ubliqua of \&. se, ab. Meliksodes agilis $\delta$, bimaculata $\delta$, nivea $\delta$. fq. vernoniae $\delta$ \&. sc, ab, oligolege. type, vernoniana of Q, se, ab, oligolege, type: Emphom.: Emplior Q. 
Short-tongued Bees (6)-Halict.: Agapostemon radiatus on, viridulus $\delta$, Chloralictus pilosus $q$, se, pruinosus $q$, se, versatus ㅇ, c, Odontalictus $\$$, c.

Diptera (3)-Bombyli.: Exoprosopa fasciata ab, Systoechus, Systropus.

Lepidoptera (16)-Nymphal.: Anosia, Argynnis; Lycaen.: Uranotes; Papilion.: Callidryas, Euphoeades, Eurymus ab, Heraclides, Jasoniades, Papilio, Pieris; Hesperi.: Epargyreus, Euphyes metacomet, Limochores manataaqua, taumas fq, Polites; Noctu.: Leucania.

\section{Convolvulaceae}

Convolvulus sepium (13:581).-Blooms May 27-September 21 ; 10 visitors observed, June 16-July 7 ; Ma, W.

Long-tongued Bees (8)-Bomb.: Bombus americanorum $q$; ab ; Ceratin.: Ceratina $\$$; Megachil.: Alcidamea truncata ${ }^{\top}$; Eucer.: Melissodes bimaculata $\delta^{2}$, Peponapis $q$, Tetralonia dilecta $q$, fq; Emphor.: Melitoma ơ $q$; Anthophor.: Anthemoessa $q$.

Short-tongued Bees (1)-Halict.: Agapostemon viridulus $q$.

Coleoptera (1)-Scarabae.: Trichius piger $\mathrm{f}, \mathrm{n}$.

Cuscuta cephalanthi.-Blooms September 15-October 3; one visitor observed, September 15; Mis, W.

Short-tongued Bees (1)-Halict.: Curtisapis coriacea 오.

Cuscuta glomerata.-Blooms July 21-September 17; one visitor observed, September 8, 17; Mis, W.

Short-tongued Bees (1)-Collet.: Colletes eulophi $q$.

Ipomoea hederacea (int).-Blooms July 23-October 26; three visitors observed, July 29-September 21; Ma, R.

Long-tongued Bees (3)-Bomb.: Bombus americanorum $\delta$ ఫ, fq, consimilis ఫ̛; Eucer.: Peponapis $\sigma^{\nearrow}$.

Ipomoea pandurata $(13: 581)$.-Blooms June 24-September 20 ; 18 visitors observed, July 11-September 9 ; Ma, W.

Long-tongued Bees (14)-Ap.: Apis; Bomb.: Bombias auri-

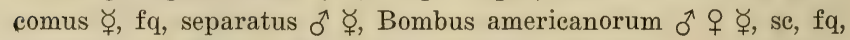
consimilis $\Varangle$; Epeol.: Triepeolus remigatus $q$; Eucer.: Cemolobus ipomoeae $\hat{\sigma} q, \mathrm{se}, \mathrm{ab}$, type, oligolege?, Epimelissodes atripes $q$, obliqua $\sigma^{\lambda}$, Melissodes bimaculata $\delta^{\lambda} q$, se, ab, Peponapis $\delta^{\lambda} q, a b$,

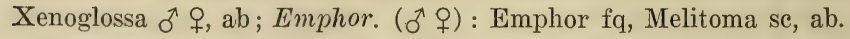

Lepidoptera (4)-Papilion.: Euphoeades, Laertias; Hesperi.: Atrytone zabulon; Sphing.: Sphinx.

Ipomoea purpurea (int).-Blooms June 25-October 26; nine visitors observed, August 16-October 7; Ma, R. 
Birds (1)-Trochil.: 'Trochilus.

Long-tongued Bees (6)-Bumb.: Bombus amerieanorum of. fig, consimilis $q$ \& : Eucer.: Melissodes himatulata $q$. P'epmapis of $q$, Xenoglossa $q$; Emphor:. Melitoma ơ $q$, se, ab.

short-tongued Bees (1) - Ilulict.: Agapostemon viridulus of.

Lepidoptera (1)_Sphing.: Deilephila.

\section{CORNACEAE}

Cornus amomum.-Blooms Ilay 29-July 26; 96 visitors observed, May 29-June 11; Mis, W.

Long-tongued Bees (9) - Ap.: Apis se, fq ; Bomb.: Bombias separatus $q$, Bombus amerieanorum $q$, se; Ceratin.: Ceratinat $f$ q , se, fq: Megachil.: Megachile brevis $q$; Xylocop.: Xylocopa $f$ se; Nomad.: Holonomada affabilis $\hat{\delta}$, fq; Eucer.: Tetralonia rosate of ; Anthophor: : Anthemoessa ơ.

Short-tougued Bees (23)-Malict. (q) : Agapostemon radiatus se, fq, virichulus se, Augochlora fervida, Chloralictus pilosus se, pruinosus, tegularis, versatus se, fq, Curtisapis forbesii, Erylacus peetoralis se, Halietus parallelus, Odontalictus se, Oxystoglosia eonfusa, similis se; Prosopid.: Prosopis illinoensis q, pyomaca of ; Andren.: Andrena platyparia $\hat{o} q$, se, sayi $q$, se, fq. Opandrena eressonii $q$, se, iq, miserabilis $q$, Trachandrena claytoniae $q$, erataegi ㅇ, heraclei $ᄋ$, se, fq, hippotes $q$.

Other Iymenoptera (15)_riphec: Isodontia apicalis, Sphex nigrioans, vulgaris; Philanth.: Corecris finitima; Vesp.: Polistes variatus; Eumen.: Leionotus anormis, arvensis, foraminatus iq. illinoensis, leucomelas, ziziae, Zendalia zenclaloides; Crabrom.: Solenius; Osybel.: Notoglossa americana, frontalis.

Diptera (31)_Syrph.: Allograpta, Chrysogaster nigripes, Eristalis dimidiatus, latifrons, transversus, Mallota cimbiciformis, Mesogramma marginata, Myiolepta nigra, Orthoncura nitida in cop, pirtipemis, Sphaerophoria, Syritta ; Bombyli.: Argyramolo albofasciata 1; Comop.: Zodion fulvifrons; Tachin.: Arohytas analis, aterrima, Belvosia bifasciata, Blepharipezal, Linnaemyia, Phorantha purpuraseress; Sarcophug.: Calliphora erythroeophala, Helieobia helicis, Sarcophaga cimbicis; Musc.: Lucilia eaesar, sericata, Pollenia, Pseudopyrellia; Anthomy.: Anthomyiella, Calythea, Phorthia acra $\mathrm{f} q$, platura $\mathrm{fq}$.

Lepidoptera (16)-Nymphal.: Areymnis, Basilarchia arrhippus, astyanax, Phyciodes, Vanessa atalanta, limntera: Lycun.: Chrysophanus, Cyaniris; Puption.: Herarlides, dasoniades, Laurtias: Ilesferi: Atrytoue zabulun, Epargyreus, Limochores taumas, Polites; C'tenuch.: Scepsis. 
Coleoptera (2)-Curculion.: Centrinus scutellum-album; Mordell.: Mordella marginata in cop.

June 1, 1911, the following 34 species and 97 individuals were taken on the flowers:

Long-tongued Bees (4:21)-Ap.: Apis. 4; Bomb.: Bombus americanorum 6 ; Ceratin.: Ceratina $10 ;$ Xylocop.: Xylocopa 1.

Short-tongued Bees (16:56)-Halict.: Agapostemon radiatus 4, viridulus 13, Chloralictus pilosus 2, tegularis 1, versatus 3, Curtisapis forbesii 1, Evylaeus pectoralis 3, Halictus parallelus 2, Oxystoglossa confusa 1, similis 2; Prosopid.: Prosopis illinoensis 1; Andren.: Andrena platyparia 4, sayi 7, Opandrena cressonii 2, miserabilis 3, Trachandrena heraclei 7 .

Other Hymenoptera (2:5)-Eumen.: Leionotus foraminatus 1; Oxybel.: Notoglossa americana 4.

Diptera (6:7)—Syrph.: Allograpta 1, Chrysogaster nigripes 1, Eristalis transversus 2; Bombyli.: Argyramoeba albofasciata 1; Sarcophag.: Sareophaga cimbicis 1; Anthomy.: Phorbia platura 1.

Lepidoptera (5:7)-Nymphal.: Argynnis 1; Papilion.: Heraclides 1, Jasoniades 3, Laertias 1; Hesperi.: Epargyreus 1.

Coleoptera (1:1)-Mordell.: Mordella marginata 1.

Cornus florida $(10: 232-3)$.-Blooms April 22-May 8; 29 visitors observed on April 23; Mis, W.

Long-tongued Bees (3)-Bomb.: Bombias separatus o; Megachil.: Osmia pumila + , se; Nomad.: Nomada illinoensis $\sigma^{\top}$.

Short-tongued Bees (15) - Halict. (q): Agapostemon radiatus, Chloralictus obscurus se, pilosus se, sparsus sc, fq, versatus, zephyrus, Curtisapis coriacea, Evylaeus foxii se, Oxystoglossa confusa se, pura se, Seladonia se, fq; Andren.: Andrena carlini $q$, Opandrena miserabilis $o^{\lambda} q, \mathrm{se}, \mathrm{fq}$, Trachandrena claytoniae $q, \mathrm{se}, \mathrm{fq}$, rugosa ${ }^{\lambda} q$.

Other Hymenoptera (2)-Vesp.: Polistes pallipes; Eumen.: Leionotus foraminatus.

Diptera (7)—Syrph.: Criorhina umbratilis, Mesogramma marginata; Empid.: Empis humilis fq; Bombyli.: Bombylius major fq, pulchellus; Lonchae.: Lonchaea aberrans.

Lepidoptera (1)-Hesperi.: Thanaos juvenalis.

Coleoptera (1) -Cerambyc.: Molorchus.

Cornus paniculata $(10: 233-5)$.-Blooms May 12-June 24; 82 visitors observed, May 12-June 8; Mis, W.

Long-tongued Bees (7)-Ap.: Apis se, fq; Bomb.: Bombias auricomus $q$, c, Bombus americanorum $q$, c; Ceratin.: Ceratina $\uparrow$ se; Nomad.: Holonomada affabilis + , superba + ; Eucer.: Tetralonia dilecta $\delta^{\pi}$ ㅇ, sc. 
Short-tongued Bees (24)-Ualict. (\$): Agapostemon radiatus se, viridulus se, Chloralietus pilosus, sparsus se, tegularis fif, versatus sp, fq. Evylacus pectoralis se, Oxystoglosat confusa se, similis; Prosopid.: Prosopis erataegi d, eulophi of bygmaea of, sayi f. ziziae of Andren.: Andrena earlini $q$, corni $q$ type, paniculatae $f$, se, platyparia $\mathcal{O} \&$, se, ab, sayi $q$, se, Opandrena eresonii $\&$, se, Trachandrena elaytoniae f. sc, fq, forbesii \&, hippotes ?. rugosa $q$, se.

Other Hymenoptera (13)-Sphec.: Chalybion, Seeliphron; Eumen.: Aneistroeerus campestris, unifasciatus, Eumenes fraternus, Leionotus foraminatus, ziziae, Monohia; Crabron.: Lindenias erraus, Solenius; Oxybel.: Notoglossa americana; P'ompril.: Ceropales fulvipes; Chalcid.: Smicra torvina.

Diptera (32) - Syrph.: Eristalis aeneus, dimidiatus, transser-us, Mallota eimhieiformis, Orthoneura nitida, Pipiza pulehella, Syrphons americanus, Tropidia mamillata; Emprid.: Empis distans, levicula, Parempis; Conop.: Oneomyia; Tachin.: Archytas analis $\mathrm{ff}$, aterrima, Belvosia bifasciata, unifaseiata, Chactophleps, Enphorocera claripennis iq. Exorista enfinis, Frontina frenchii, Paradidmya, Phorantha purpuraseens, Spallanzania; Sarcophag.: IIelicobia helicis, Sarcophaga bullata; Muse.: Graphomyia, Lueilia eacsar, serieata, Morellia; Anthomy.: Anthomyiella, Phorbia acra; Ortal.: Camptoneura.

Lepidoptera (2)-Nymphal.: Vanessa atalanta; P'tpilim.: Iphiclides.

Coleoptera (8)-Cerambyc.: Strangalia; Lampyr.: Poulabrus tomentosus iq]. Telephorus flavipes fq]; Mordell.: Mordella marginata fo. Pentaria; Scurabae.: Euphoria fulgida. Trichius affinis in cop, piger fq.

\section{Cructarerae}

Arabis dentata.-Blooms April 5-May 31; 48 species and 2.54 individuals observed, April 24-May $15 ; \mathrm{Mi}, \mathrm{W}$.

Long-tongued Bees (12:54)-Ap.: Apis se, 34 : Bomb.: Bombias separatus \&, 2, Bombus impatiens 9,3 ; Ceratin.: Ceratina of, 1 , Zaodontomerus 8,2 ; Meguchil.: Osmia atrisutris 9,2 , pumila 8,1 ; Nomad.: Gnathias cuneatus of 9,2 , ovatus $q, 2$, santhoparius $\delta, 2$, Xanthidium lutenlum Q. 1; Eucer.: Tetralmia belfragei of \&. 2.

Short-tongual Bees (25:175) - Halict. 19): Agapostemsil radiatus 3, Augochlora viridula 25, Chloralietus eaeruleus 1, obseurus 2. sparsus se, 7 , tegularis se, 1 , versatus 11 , sephyrus 9. Curtisapis eoriacen 7, Evyleaus foxii si, 27, pectoralis se, 7, 4-maculatus 1 . Halictus lerouxii 2, Oxystoglosen confusa 8, pura 12, simllis se, 6 . 
Seladonia se, 4; Sphecodini : Proteraner ô, 6 ; Prosopid.: Prosopis sayi $\delta, 1$; Andren.: Andrena carlini $q, 1$, geranii $\delta$, 3, nasonii $q$, se, 9, Opandrena cressonii $\delta^{\top}+$, sc, 10 , miserabilis + , 1, personata 주우, 13.

Other Hymenoptera $(2: 2)$-Eumen.: Leionotus foraminatus 1; Sapyg.: Sapyga 1.

Diptera (9:21)—Syrph.: Mesogramma geminata 4, Syrphus americanus f, 6, ribesii f, 5, torvus f, 1; Empid.: Empis humilis 1, nuda 1, Pachymeria 1; Bombyli.: Bombylius major 1; Tachin.: Siphona 1.

Arabis laevigata.-Blooms April 10-May 18; 13 species and 53 individuals observed, April 23, 27, May 5; Mi, W.

Long-tongued Bees (4:8)-Ceratin.: Ceratina + , 4, Zaodontomerus $\delta$, 2; Megachil.: Osmia pumila $q, 1 ;$ Nomad.: Gnathias ovatus $q, 1$.

Short-tongued Bees ( $7: 40$, ㅇ)-Halict.: Chloralictus obseurus 1, versatus se, 9, Evylaeus 4-maculatus 1, Oxystoglossa confusa se, 8 , pura 1; Andren.: Andrena arabis se, 18, type, nasonii se, 2.

Diptera (2:5)—Syrph.: Allograpta f, 1; Tachin.: Siphona 4.

Arabis virginica.-Blooms March 20-May 15; 11 visitors observed, April 20, May 3, 8; Mi, W.

Long-tongued Bees (1)-Nomad.: Gnathias ovatus + .

Short-tongued Bees (9)-Halict. ( $($ ) : Agapostemon radiatus, Chloralictus versatsus, zephyrus, Halictus lerouxii, Oxystoglossa confusa, similis, Seladonia; Andren.: Opandrena cressonii o ; miserabilis $\sigma^{7}$.

Diptera (1)-Anthomy.: Anthomyiella.

Capsella bursa-pastoris (int).-Blooms March 20-December 1; 60 visitors observed, April 16-June 1; Mi, W.

Long-tongued Bees (4)-Ap.: Apis fq; Megachil.: Prochelostoma $\sigma^{\lambda}$; Nomad.: Nomada parva $q$; Eucer.: Tetralonia dilecta $\delta^{\top}$.

Short-tongued Bees (22)-Halict. (q): Chloralictus coreopsis sc, cressonii, sparsus sc, ab, tegularis se, versatus se, zephyrus sc, Curtisapis forbesii sc, Dialictus se, Evylaeus foxii se, fq, pectoralis se, Halictus lerouxii se, Odontalictus, Oxystoglossa similis se, fq, Seladonia se; Prosopid.: Prosopis pygmaea $\sigma^{\hat{*}}$; Andren.: Andrena arabis $q$, se, fq, nasonii $q$, se, Opandrena cressonii $\delta q$, se, fq, miserabilis $\lambda$, personata $\widehat{\delta} q, \mathrm{se}, \mathrm{fq}$, Trachandrena claytoniae $\hat{\delta} q$, se, forbesii $\delta^{\lambda}$.

Other Hymenoptera (1)—Vesp.: Polistes pallipes. 
Diptera (27)-Syrph.: Allograpta, Eristalis dimidiatus sf, meigenii fq, Ifelophilus latifrons, similis, Mallota cimbiciormis, Melanostoma mellinum, Mesogramma geminata, marginata, Sphacrophoria, Syritta st; Bombyli: Ahlriehia sf: Conop.: Oneomyia, Zodion fulvifrons; Tachin.: Apinops, Gonia capitata, Ciymnopresopa, Peleteria, Siphona fq ; Sureophag.: Cynomyia if, Melicobia helieis, Sareophaga simuata; Muse.: Lueilia catsar, sericata, sylvarum fq; Anthomy.: Phorbia, acra, platura.

Lepidoptera (6)-Vymphal.: Chardidyas, Phyeiodes; Papilion.: Pontia; Mesperi: Pholisom eatullus, Polites, Thanaos martialis.

The first 100 individuals taken on the flowers, May 11, 1:3, 15, 17, 1916, showed species and individuals as follows:

Short-tongued Bees (12:63)-Malict.: Chloralintus moopis 1, sparsus 26, tegularis 1, versatus 2, zephyrus 1, Dialietus 1, Erylateus foxii 3, pectoralis 9, Oxystoglossa similis 2; Andren.: Andrena arabis 7 , Opandrena eressonii 1 , personata 9 .

Diptera (13:37) - Syrph. Eristalis meigenii 5, Mallota cimbiciformis 1, Mesogramma geminata 1, marginata 4, Syritta 4 ; Bombyli: Aldrichia 1; Tachin.: Gymnoprosopa 1. Siphema 6; Musc: Lucilia eaesar 2, serieata 1, sylvarum 7 ; Anthomy.: Phorbia acra 3 , platura 1 .

Cardamine bulbosa $(2: 298-9)$.-Blooms April 2.2-1Iay 30:49 visitors observed, May 2-21; Mi, W.

Long-tongued Bees (14)-Bomb.: Bombias auricomus ?; Cerutin.: Ceratina of o. se, fq. Zaodontomerus of; Megachil.: Alei-

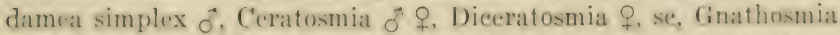

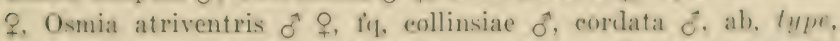
pumila of $q$, se, ab; Nomad.: Holonomada superba of $q$. Nounada denticulata ơ; Eucer.: Tetralonia dilecta ơ 우.

Short-tongued Bees (14)-Mulict. (f): Chloralietus pilusus se, pruinusus se, fq. versatus se, fq. Evylieus pectoralis se. fi. Difontalietus, Oxystoglosa confusa, similis, Scladonia fị; Prosoprid.: Prasopis pygmaca of Andren.: Andrena arahis of se. ah, sayi fo. Iomelissa $q$, Opandrena miserabilis $q$, personata 0 .

Diptera (13)_stratiomy.: Nemotelus; Syrph.: Mesoramma mareinata, Orthoneura pictipennis, Sphaerophoria : Empril.: Empis avida, nuda, otiosa; Bombyli.: Bombylius atricejs fo, major: Conop.: Myopa vesiculoni, Zodion fulvifrons; Muse.: Turilia raesar; Anthomy.: Phorbia aera.

Lepidoptera (8) - Nymphal.: Phyeiodes, Vanessa atalanta: Lycuen.: Everes: Papilion.: Eurymus. Pieris, Xanthidia ; Ilesperi: Epargyreus, Pholisora catullus. 
Dentaria laciniata.-Blooms April 2-May 2; 39 visitors observed, April 2-28; Mi, W.

Long-tongued Bees (19)-Ap.: Apis se, fq.; Bomb. (q) : Bombias separatus, Bombus americanorum, impatiens, ridingsii; Ceratin.: Ceratina $\delta^{\lambda} ;$ Megachil.: Centrosmia $\delta \uparrow$, Ceratosmia $\delta$, Osmia pumila $\delta^{\lambda}$; Nomad.: Gnathias cuneatus $\delta^{\lambda}$, ovatus $\delta^{\lambda}$, Nomada cressonii $\sigma^{\lambda}$, illinoensis $\sigma^{\lambda}$, sayi $\delta^{\lambda} q$, fq, Phor $\sigma^{\lambda}$, Xanthidium dentariae $\delta^{\lambda}$, fq, type, luteoloides $\sigma^{\lambda}$, fq, luteolum $\sigma^{\lambda}, \mathrm{fq} ;$ Eucer.: Tetralonia belfragei $\delta^{\lambda}$ ㅇ.

Short-tongued Bees (14)-Halict. (q): Agapostemon radiatus, Chloralictus pilosus, sparsus se, versatus, Evylaeus foxii, Halictus lerouxii sc, Odontalictus; Andren.: Andrena carlini $\sigma^{\top} q$, fq, pruni $\delta^{\lambda}$, sayi $\delta^{\lambda}+$, fq, tridens $\delta^{\lambda}$, Opandrena cressonii $q$, miserabilis $\delta^{\lambda} q$, Trachandrena rugosa $0^{\lambda}$ ㅇ․

Diptera (3)- Syrph.: Sphaerophoria f, n; Empid.: Empis humilis; Bombyli.: Bombylius major.

Lepidoptera (2)-Lycuen.: Everes; Papilion.: Iphiclides.

Coleoptera (1)-Nitidul.: Cereus $\mathbf{f}, \mathrm{fq}, \mathrm{n}$.

Lepidium virginicum.-Blooms April 12-November 7; 45 visitors observed, May 17-June 9 ; Mi, W.

Long-tongued Bees (2)-Ceratin.: Ceratina o $o$, fq, Zaodontomerus $\delta$, fq.

Short-tongued Bees (15)-Halict. (q) : Agapostemon radiatus, Chloralictus coreopsis, sparsus se, tegularis se, versatus, Evylaeus areuatus, pectoralis sc, ab, Odontalictus, Oxystoglossa similis se, fq, Seladonia; Prosopid.: Prosopis pygmaea $\delta^{\lambda} q$, sayi $q$, ziziae $\delta^{\lambda} q$, fq; Collet.: Colletes eulophi ơ; Andren.: Opandrena personata $q$, se.

Other Hymenoptera (6)-Eumen.: Leionotus clypeatus, histrionalis, illinoensis; Oxybel.: Notoglossa frontalis; Pompil.: Anoplius illinoensis, Psammochares tenebrosus.

Diptera (21)-Stratiomy.: Nemotelus; Syrph.: Eristalis dimidiatus, Helophilus similis, Mesogramma marginata ab, Paragus bicolor, tibialis, Syritta; Conop.: Zodion fulvifrons fq; Tachin.: Apinops, Cistogaster occidua, pallasii, Gymnosoma, Leucostoma atra, Ocyptera tenuis, Phorantha purpurascens; Sarcophag.: Helicobia helicis; Musc.: Lucilia caesar, Pseudopyrellia; Anthomy.: Limnophora, Phorbia acra ab, platura ab.

Lepidoptera (1)-Papilion.: Pontia.

May 20, June 1, 1909, 166 individuals belonging to 34 species were taken on the flowers:

Long-tongued Bees (2: 11)-Ceratin.: Ceratina 5, Zaodontomerus 6. 
Short-tongued Bees (12:35)-Maliet.: Agapostemon radiatus 1, Chloralietus coreopisis 1 , tegularis 1 , versatus 1 , Evylaeus arcuatus 1 , peetoralis 6, Oxystoglossa similis 6 , s.ladonia 4; Prosogrid.: P'rosopis pygmaea 4, sayi 2., ziziae 5; Andren.: Opandrena personata 3.

Other Ilymenoptera (4: 6)-Eumen.: Lrionotus elypuatus 1, histrionalis 3 ; Pompil.: Anoplius illinoensis 1. Psammoehares tenebrosus 1.

Diptera (16: 114)-Stratiom!y.: Nemotelus 1; Symph.: Eristalis dimidiatus 1, Helophilus, similis 1, Mesogramma margiuata 33, Paragus bieolor 1, tibial is 1 ; Conop.: Zodion fulvifrons 6; Tuchin.: Apinops 1, Cistogaster occidua 1, pallasii 1, Oeyptera temuis 1; Sarrophag.: Helicobia helicis 1; Muse.: Pseudopyrellia 1; 1 n. thomy.: Limnophora 1, Phorbia acra 52, platura 12.

Radicula obtusa.-Twenty-three visitors wherved, May 1, 3, 8; Mi, Y.

Long-tongued Bees (3)-.1p.: Apis Iq; Megnehil.: Aleidamea simplex $q$; Nomad.: Nomada illinoensis $q$.

Short-tongued Bees $(11, q)$ - Hulict.: Chloralictus albipennis, pilosus, versatus se, fq, zephyrus, Evylaeus areuatus, Odontalietus, Oxystoglossa confusa, similis $\mathrm{fq}$. Seladonia fq; Andren.: Andrena erythrogastra, Opandrena eressonii.

Other Hymenoptera (3)-Crabron.: Clytoehrysus; Tiphi.: Tiphia clypeata; Ichnoumon.: Metopius terminalis.

Diptera (6)-Syrph.: Sphaerophoria, Syrphus amerieanus; Tachin.: Peleteria ; Sarcophag.: Sareophaga sinuata; Musc.: Lueilia sylvarum; Anthomy.: Limnophora.

Radicula palustris.-Blooms May 6-October $16 ; 6$ species and 22 individuals observed, May 31 , June $3 ;$ Mi, Y.

Short-tongued Bees $(4: 1 \mathrm{~h}, \mathrm{q})$-Haliet.: Chloralietus tegularis se, 2, versatus se, 9, zephyrus 2, Evylaeus pectoralis 5 .

Diptera $(2: 4)$-Syrph.: Mesogramma marginata sf, $3 ; \mathrm{An}$ thomy.: Phorbia platura 1.

Sisymbrium canescens.-Blooms April 13-June 8; 13 vivitors observed, May 2, 3; Mi, Y.

Short-tongued Bees (11)-Haliet. (9): Chloralietus pilesus se',

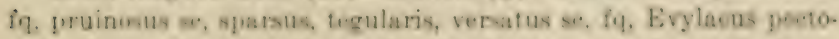
ralis, Oxystoglose similis se. fy. Seladonia oc; Andren.: Andrena nasonii $\delta$, Opandrena cressonii $q$, se, personata $\delta$, se.

Diptera (2) - Techin.: Fymnosoma; Anthamay. : Pherbia anra. 


\section{Cucurbitaceae}

Cucurbita melopepo? (cult).--One visitor observed, July 11, September 21; Ma, Y.

Long-tongued Bees (1)-Eucer.: Xenoglossa ô.

Cucurbita pepo (cult).-Blooms July 3-October 4; 6 visitors observed, July 3-September 21; Ma, Y.

Long-tongued Bees (6)-Ap.: Apis se, fq; Bomb.: Bombus americanorum $\sigma^{\lambda} \not{\psi}$, se, fq, impatiens $\not{\phi} ;$ Eucer.: Melissodes bimaculata $q$, Peponapis $\sigma^{\lambda}+$, se, ab, oligolege, Xenoglossa $\delta^{\wedge} q, \mathrm{se}, \mathrm{ab}$.

Echinocystis lobata.-Blooms July 26-September 27; 21 visitors observed on August 1: Pol, W.

Long-tongued Bees (2)-Ap.: Apis; Eucer.: Melissodes bimaculata q.

Short-tongued Bees (1)-Halict.: Chloralictus versatus $q$, sc.

Other Hymenoptera (3)-Scoli.: Elis 5-cincta, Scolia bicincta; Larr.: Tachytes elongatus.

Diptera (15) -Syrph.: Allograpta ab, Mesogramma geminata, marginata, Milesia, Syritta fq, Syrphus americanus, ribesii; Sarcophag.: Sarcophaga assidua; Musc.: Lucilia eaesar, sericata, Musca, Phormia regina, Pseudopyrellia; Anthomy.: Ophyra, Phorbia acra.

Sicyos angulatus.-Blooms August 18-September 25; 17 visitors observed, August 21-31; Pol, Y.

Long-tongued Bees (3)-Ap.: Apis fq; Bomb.: Bombus impatiens $\not$; Eucer.: Melissodes bimaculata $\circ$, se.

Other Hymenoptera (9)-Sphec.: Ammobia ichneumonea, Chalybion, Sceliphron; Bembic.: Sphecius; Vesp.: Polistes annularis, pallipes fq, rubiginosus, Vespula germanica, maculata.

Diptera (5)—Syrph.: Mesogramma marginata, polita; Tachin.: Winthemia; Musc.: Lucilia caesar, Musca.

\section{Dipsaceae}

Dipsacus sylvestris (int).-Blooms July 1-August 29; 10 visitors observed on July 3, 28; Mas, R.

Long-tongued Bees (2)-Bomb.: Bombus americanorum $\Varangle$; Eucer.: Melissodes bimaculata $ð$.

Short-tongued Bees $(2$, q) -Halict.: Agapostemon radiatus sc, viridulus.

Diptera (1)-Bombyli.: Exoprosopa fasciata fq.

Lepidoptera (5)-Nymphal.: Anosia, Vanessa huntera; Papilion.: Jasoniades, Pontia ; Hesperi.: Thanaos martialis. 


\section{Ebenacear}

Diospyros virginiana.-Blooms May 227-June 2.5; 29 species and 279 individuals observed, May 30-June 2.7; Ma, Y.

On staminate flowers $(17: 127)$ :-

Long-tongued Bees (4:8)-Bomb.: Bombias separatus of, se. 2, Bombus impatiens o 8. se, 4; Coralin.: Ceratina ? se, 1, n; Nomol.: Holonomada affabilis $q, 1$.

Short-tongued Bees $(10: 109, \mathrm{n})$-Hulict.: A rapustruon radiatus $8 . \mathrm{se}, 1$, Augrehlora fervida o, se, 3, viridula of se, 5, Chloralietus sparsus $q, e, 2$, versatus $q, c, 7$, zephyrus $\&, s r, 1$, Curtisapi coriacea ?. se, 3, furbesii \&. e. 1, fuseipennis ? se, s.5, Oxystoglossa similis $q, \mathrm{e}, 1$.

Diptera (3: 10, f, n) -Syrph.: Allograpta 1, Syrphus amerieanụs 1 , ribesii 8 .

On staminate and pistillate flowers $(9: 146)$ :-

Long-tongued Beres (6: 1:35) - Ap.: A pis se, is ; Boml).: Bumbus americanorum \%, 6; Megarhil.: Osmia pumila f. 2. Megachile

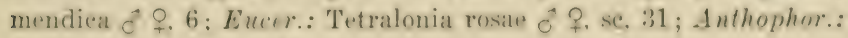
Anthemoessa ${ }^{\top}$ 우, se, 15.

Short-tongued Bees (2: 6, \%, se)-Helict.: Agapostemon viridulus 4, Oxystoglossa confusa 2.

Lepidoptera $(1: 2)-$ IIesperi.: Euphyes verna 2.

On pistillate flowers $(3: 6)$ :-

Long-tongued Bees (1:2)-Bomb.: Bombias auricomus \&. 2.

Short-tongued Bees (1:1)-Halict.: Oxystnglosia pura of 1, n. Lepidoptera (1:3)-Hesperi.: Limochores manataaqua 3, n.

\section{EUPHORBiaceat}

Euphorbia corollata $(9: 74-5)$.-Blooms May 24-Sejtember 27 ; 52 visitors observed, July 6-August 22 ; Pol, W.

Short-tonerued Bees (7)-Halict. (f): Chloralietus pilusus se. sparsus, versatus se. fq. Seladonia; Spheeodini: Spheendes arvensis

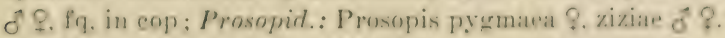

Other Hymenoptera (22)-Sphec.: Prionmys atrata, Seeliphron: Brmbir.: Megastizus; Ścoli.: Elis nemeta fq; Philanth.: Euerereris; Fesp.: Polistes pallipes, variatus; Eumen.: Aneintrocerus campestris. Leionotus dorsalis: Crobron.: Anaerabro. Lindenius errans, Protothyreopus higemims: Orybel. Oxyledus packardii, 4-notatus; Pemphredon.: Mimesa ; Larr.: Larropsis; Pumpal.: Prioenemoides fulvicornis. Pammachares r.lativus; Chrysid.: Hedychrum wiltii, IIolopyga; Thalcil.: Leurospis; Ithneumun.: Ceratosoma. 
Diptera (21)—Syrph.: Allograpta ab, Mesogramma polita, Orthoneura nitida, Paragus bicolor fq, tibialis, Pipiza pulchella, Sphaerophoria ab, Syritta ab; Bombyli.: Anthrax alternata, Exoprosopa fasciata, fascipennis; Tachin.: Alophora aeneoventris, Cistogaster pallasii fq, Cyphocera, Pachyophthalmus signatus, Phorantha purpurascens, Senotainia trilineata fq, Trichopoda pennipes; Sarcophag.: Helicobia helicis; Musc.: Morellia, Pseudopyrellia.

Lepidoptera (1)-Papilion.: Pontia.

Hemiptera (1)-Core.: Chariesterus.

Euphorbia preslii.-Blooms June 30-October 26; 7 visitors observed, August 11, 24; Mi, W.

Short-tongued Bees (1)-Halict.: Chloralictus tegularis $q$, sc.

Other Hymenoptera (3)-Oxybel.: Notoglossa frontalis; Philanth.: Cerceris finitima; Chrysid.: Hedychrum parvum.

Diptera (3)—Syrph.: Mesogramma marginata, Syritta, Bombyli.: Sparnopolius.

\section{Fumariaceae}

Dicentra cucullaria $(2: 125-6)$.-Blooms March 21-May 5; 23 visitors observed, April 9-30; Ma, W.

Long-tongued Bees (14)-Ap.: Apis c, ab, $\mathrm{n} ;$ Bomb. (ㅇ): Bombias auricomus $f q$, separatus $f q$, Bombus americanorum $f q$, consimilis, impatiens, ridingsii; Megachil.: Centrosmia $\widehat{\delta} \stackrel{q}{q} \mathbf{f}$, Ceratosmia $\sigma^{\lambda}$ 웅, Osmia collinsiae $\sigma^{\top}$; Eucer.: Tetralonia belfragei $\sigma^{\lambda}$

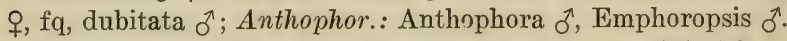

Short-tongued Bees (1)-Andren.: Andrena carlini $\delta$ o.

Diptera (1)-Bombyli.: Bombylius major fq.

Lepidoptera (6, n)-Nymphal.: Anosia, Vanessa atalanta; Papilion.: Iphiclides, Jasoniades, Pieris; Hesperi.: Thanaos martialis.

\section{Gentianaceae}

Frasera carolinensis $(6: 48,8: 142)$.-Blooms May 24-June 22 ; 7 visitors observed, May 30-June 13; Ma, Y.

Long-tongued Bees (4)-Ap.: Apis ab; Bomb.: Bombias separatus $q \varnothing, \mathrm{q}, \mathrm{b}$; Bombus americanorum $q$; Anthophor.: Anthemoessa o $q$, fq.

Short-tongued Bees (1)-Halict.: Curtisapis coriacea + , sc, $\mathbf{n}$.

Other Hymenoptera (1)-Vesp.: Polistes pallipes.

Lepidoptera (1)-Hesperi.: Thorbyes pylades $\mathbf{n}$.

Gentiana andrewsii (13:577-8).-Blooms September 9-October $20 ; 2$ visitors observed, September 23, 29 ; Ma, R. 
Long-tongued Bees (1)-Bomb.: Bombus americanorum of $q \not$. ab.

Coleoptera (1) - Melo.: Epicauta pennsylvaniea gn, $n$.

Gentiana puberula $(8: 139-40)$.- One visitor observed, September $29 ; \mathrm{Ma}, \mathrm{R}$.

Long-tongued Bees (1)-Bomb,: Bombus americanorum of ?.

Sabatia angularis.-Blooms July 11- September 9; ; visitors observed, August 2, 5 ; Ma, R.

Long-tongued Bees (2)-Ceratin.: Ceratina q, se; Megachil.: Megachile brevis ?.

Short-tongued Bees (1)-IIalict.: Oxystoglossa similis $q$, e, n.

\section{Geraniacene}

Geranium carolinianum (6 : 272).-I3looms May 21-July 1:3; 13 visitors observed, May 21, June 10; Mi, R.

Long-tongued Bees (3)-Megachil: Aleidamea simplex of $q, \mathrm{f}_{\mathfrak{1} 1}$, Diceratosmia ; Eucer.: Tetralonia dilecta ㅇ.

Short-tongued Bees (7)-Halict. ( $($, se) : Agapostemon rarliatus, Chloralictus sparsus, tegularis, Evylaeus pectoralis, $\mathrm{f}(\mathrm{l}, \mathrm{Oxy}-$ stoglossa ronfusa fig; Prosopid.: Prosopis ziziae o; Panurg.: Heterosarus $q$, se.

Other Hymenoptera (1)-Eumen.: Leionotus illinoensis.

Diptera (2)-Syrph.: Mesogramma marginata fq; Tachin.: Phorantha purpuraseens.

Geranium maculatum $(2: 299-300)$.-Blooms April 13-June 4; 44 visitors, April 23-May 24; Мa, R.

Long-tongued Bees (24)-Ap.: Apis; Bomb. (q): Bombias separatns, Bombus amerieanorum, eonsimilis, impatiens, ridingsii, Psithyrus variabilis; Cerutin.: Ceratina o $\sigma^{\circ}$; Stelid.: Microst. is $\$, n$; Megachil.: Trypetini: Prochelostoma of o. n; Osmini : Aleidamea simplex $\hat{\delta}$, Ceratosmia $o$, Monilosmia $d$ o. Osmia atriventris $\hat{\delta}$, pumila 9 ; Coelioxyini: Coelioxys sayi $\delta$ \$; Nomud.: Centrias americanns $\delta$, Holonomada superba $\delta$ f Nomada denticulata $q$, Xanthidium luteolum $\$$; Eucer.: Tetralonia belfragei 8 , dilecta $\delta^{7}+$, fq, rosac of; Anthophor.: Anthophora ․

Short-iongued Bees (11)-Ilalict. (2): Agapostemon viridulus, Augochlora viridula, Chloralietus pilosus n, versatus n, Curtisapis coriacea, Halietus lerouxii se, Oxystoglossa similis n; Andren.: Andrena geranii of $\%$. fq, type, illinoensis of, n, Ptilandrena g. maeulati $\delta^{\wedge} q, \mathrm{se}, \mathrm{ab}$, oligolege, type, polemonii $\delta^{\lambda} q, \mathrm{fq}, \mathrm{n}$. 
Diptera (5, n)-Syrph.: Helophilus latifrons f; Empid.: Empis distans fq, humilis, otiosa, Pachymeria ab.

Lepidoptera (4)-Lycaen.: Everes; Papilion.: Eurymus; Hesperi.: Thanaos martialis; Sphing.: Hemaris thysbe.

\section{Gramineae (anemophilous)}

Panicum virgatum.-One visitor observed, July 30.

Short-tongued Bees (1)-Halict.: Chloralictus versatus $\nmid$, c, 5. Poa pratensis.-One visitor observed, June 9.

Short-tongued Bees (1)-Halict.: Chloralictus sparsus $q$, c, 4.

Zea mays.-One visitor observed, September 1.

Long-tongued Bees (1)-Ap.: Apis c, 2.

\section{Hydrophyllaceae}

Ellisia nyctelaea $(6: 49-50)$.-Blooms April 20-June 21; the first 100 individuals, representing 26 species, were taken on May 8-23; Mi, W.

Long-tongued Bees (8:30)—Bomb.: Bombus consimilis ㅇ, 1; Ceratin.: Ceratina $\delta^{\lambda}+, 9$, Zaodontomerus $\sigma^{\lambda}, 8$; Megachil.: Prochelostoma $\delta^{\lambda}+$, 5, Osmia atriventris $\delta^{\lambda}+$, se, 2, pumila $\delta, 1$; Nomad.: Gnathias cuneatus + , 2; Eucer.: Tetralonia dilecta 0 , 2.

Short-tongued Bees (11:55)-Halict. ( $(q)$ : Chloralictus obscurus 7, sparsus se, 4, tegularis 2, versatus 2, zephyrus 1, Evylaeus pectoralis 1, 4-maculatus 10, Oxystoglossa confusa 8, Seladonia se, 7; Andren.: Iomelissa $\uparrow, 1$, Opandrena personata $\delta \uparrow$, se, 12.

Diptera (7:15)—Syrph.: Mesogramma geminata f, 1, marginata f, 4, Pipiza femoralis f, 1, Rhingia 2; Bombyli.: Bombylius major 2, pulchellus 1; Tachin.: Siphona 4.

Hydrophyllum appendiculatum (13:579-80).-Blooms May 2- June 19; 46 visitors observed, May 3-June 19; Ma, R.

Long-tongued Bees (20)-Ap.: Apis sc, ab; Bomb.: Bombias auricomus $q$, separatus $q \not$, se, ab, Bombus americanorum $q$, ab, consimilis $q$, impatiens $q \not$, fq, ridingsii $q$ $\Varangle, \mathrm{se}, \mathrm{ab}$, Psithyrus variabilis $\uparrow$; Ceratin.: Ceratina $q$; Megachil.: Trypetini : Prochelostoma ᄋ, sc ; Osmiini : Alcidamea simplex $\sigma^{\lambda}$, Ceratosmia + , ab, Diceratosmia $q$, se, Hoplitis $\sigma^{\lambda}+$, sc, Osmia pumila + , sc, ab; Megachilini: Megachile brevis $\delta^{\top}$; Nomad.: Holonomada affabilis $ᄋ$; Eucer.: Tetrailonia belfragei $\delta^{\lambda}+$, se, ab, dilecta $\delta^{\lambda}$, fq, rosae $\delta^{\lambda}+$, fq.

Short-tongued Bees (10)-Halict. () : Agapostemon radiatus sc, Augochlora viridula, Chloralictus pilosus, versatus, Curtisapis coriacea sc, ab, Evylaeus pectoralis, 4-maculatus, Oxystoglossa confusa se, ab, Andren.: Andrena earlini $q$, geranii $\sigma^{\lambda} q$, sc, ab. 
Other IIymenoptera (2) - Eumen.: Aneistrocerus tigris, Leionotus foraminatus.

Diptera (10)-Syrph.: Criorhina intersistens, Eristalis flavipus, Thingia sf, ab. Sphegina rufiventris f, n. Xylota chalylea ; Empid.: Empis loripedis, nula ab; Bombyli.: Aldrichia f. n; Conop.: Stylogaster biamulata: Anthomy.: Hy.lenyia inornata.

Lepidoptera (4)-Nymphal:: Charidryas; Hesprri: Amblyseirtes, Atrytone zabulon, Pholisora hayhurstii.

May 1s. 1909, the following 7 species and 22 individuals wre taken on the flowers:

Long-tongued Bees (7:22)-Bomb.: Bombias aurieomus 1, separatus 8. Bombus amerieanorum 2, ennsimilis 1, impatiens 2; Eucer.: 'T'etralonia belfragei 4 , dilecta 4 .

Hydrophyllum virginianum (13:579).-Bloms May 6-.June $1 ; 19$ visitors observed May 8-27; Ma, W.

Long-tongued Bees (12)-Bomb.: Bombias separatus ? ab, Bombus americanorum $\&$ \& $a b$, eonsimilis \&. fq, impatiens \&. fif.

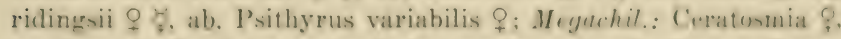
se; Nomal.: Gnathias ovatus $q$, fq; santhoparius of : Euerr.: Tetralonia belfragei $q$, se, dileeta $\delta$, fy; Anthophor.: Anthemoessa $d f$

Short-tongued Bees (3)-IInlict. ( $($ ) : Curtisapis enriacea se, Oxystoglossa confusa $e, n ;$ Andren.: Andrena geranii of \&, se. fq.

Other Iymenoptera (2)-Eumen.: Ancistrocerus tigris, sueking at holes made by Leionotus, Leionot us foraminatus, perforating.

Diptera (2)-syrph.: Rhingia i, n; Bombyli.: Bombylius atriceps.

May 18, 1909, the following 6 sperees and 46 individuals were taken on the flowers:

Lone-tongued Bees $(6: 46)$-Bumb.: Bombias separatus 25, Bombus americanorum 5, consimilis 22, impatiens 2, ridingsii 2; Eucer.: Tetralonia dilecta 7 .

\section{Hypericaceae.}

Hypericum ascyron.-Blooms July 3-August 7 : 2 visitms ob. served, July 5; Ma, Y.

Long-tongued Bies $(2,8, e)-B r m b$.: Bombus auerieanorum 4. consimilis 4 .

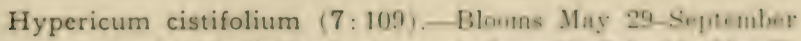

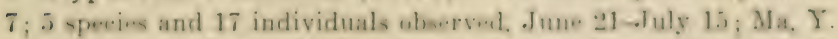

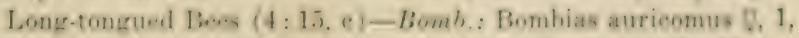

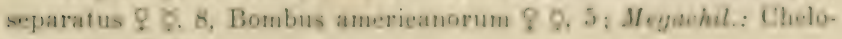
stomoides +1. 
Short-tongued Bees (1:2)-Halict.: Agapostemon viridulus + , c, 2.

Hypericum punctatum.-Blooms June 26-September 7; 3 visitors observed, June 29, July 15, 30; Mi, Y.

Long-tongued Bees (1)—Bomb.: Bombus americanorum $\not$, c.

Short-tongued Bees (2, $q$, c)-Halict.: Chloralictus pilosus, versatus.

\section{IRIDACEAE}

Iris hexagona.-Blooms June $8-20 ; 2$ visitors observed, June $10 ; \mathrm{Ma}, \mathrm{R}$.

Long-tongued Bees (2)-Bomb.: Bombus americanorum $q, 1$; Anthophor.: Anthemoessa $01,1$.

Iris versicolor $(9: 80)$.-Blooms May 17-June 14; 10 visitors observed, May 20-June 5; Ma, R.

Long-tongued Bees (5)-Bomb. (q) : Bombias auricomus, separatus, Bombus americanorum fq ; Eucer.: Tetralonia dilecta $\delta_{0} q$, se, ab, rosae $\sigma^{j}$.

Short-tongued Bees (1)-Halict.: Chloralictus versatus 우, c, n.

Lepidoptera (3, n)-Lycaen.: Chrysophanus; Hesperi.: Limochores taumas, Polites.

Coleoptera (1)-Scarabae.: Trichius piger $n$.

Sisyrinchium angustifolium.--Blooms April 18-June 6; 15 visitors observed, May 10-23; Mi, W.

Long-tongued Bees (4)-Bomb.: Bombias auricomus $q$, separatus $q$; Megachil.: Osmia pumila $q$; Nomad.: Holonomada superba $\sigma^{7}$.

Short-tongued Bees (7, ᄋ)-Halict.: Chloralictus pilosus sc, pruinosus, versatus se, ab, Oxystoglossa confusa se, ab, similis, Seladonia sc, fq; Andren.: Opandrena cressonii sc.

Diptera (4)-Syrph.: Helophilus latifrons, Mesogramma marginata, Sphaerophoria; Bombyli.: Bombylius atriceps.

Sisyrinchium gramineum.-Blooms May 11-July 1; 1 visitor observed, May 28; Mi, R.

Short-tongued Bees (1)-Halict.: Oxystoglossa similis 오 sc.

\section{LABIATAE}

Agastache nepetoides (14:115-6).-Blooms July 24-September 28; 15 visitors observed, August 2-September 2; Mas, Y.

Long-tongued Bees (6)-Ap.: Apis ab; Bomb.: Bombias separatus $\sigma^{\lambda} \not{\phi}, \mathrm{se}, \mathrm{fq}, \mathrm{Bombus}$ americanorum $\delta^{\lambda} \not{\phi}, \mathrm{se}, \mathrm{ab}$, consimilis ${ }^{\lambda}$, fq, impatiens $\not$, sc, ab; Eucer.: Melissodes bimaculata $\delta^{7}$ ㅇ. 
Short-tongued Bues $(3, \mathrm{n})$-Ilalict. (q) : Chloralictus sparsus c, ab, versatus c, Oxystoglossa pura.

Other Hymenoptera (1)-Sphec.: Sphex nigrieans.

Diptera (3)-Syrph. ( 1, n) : Mesogramma geminata, Syritta; Bombyli.: Exoprosopa fusciata.

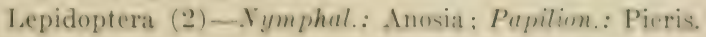

Agastache scrophulariaefolia $(14: 116)$. - Bloms Auenst †September 2s; 5 visiturs observed. Auenst :31. September 1; Mas, R.

lometongued Bees (4) -...1p.: Apis iq : Bomb.: Bombias auri-

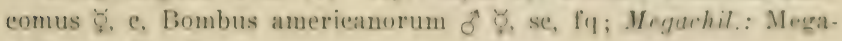
chile mendica ㅇ.

Diptera (1)-Bombyli.: Exoprosopa faseiata fq.

Blephilia ciliata $(14: 113-4)$.-Blnoms IIay 15-July $4 ; 76$ visitors observed, June 1-July 5; Mas, R.

Long-tongued Bees (25)-Ap): Apis ab: Bomb. (q) : Rombias auricomus se, separatus, Bombus amerieanorum se, fq. comsinilis, impatiens, Psithyrus variabilis; Ceratin.: Ceratina f. se, ab; Megachil.: Trypetini: Neotrypetes barbatus \&. se, fq. truncatus ?. sc, ab; Osmini: Alcidamea simplex $\%$, se, Diceratosmia ?. Moplitis o. Osmia distincta $q$; Megachilini: Cyphopyga $d$, fu. Megarhile

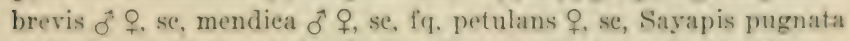

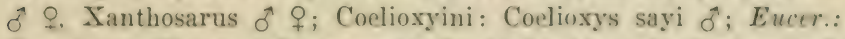
Tetralonia dileeta of + . se, rosale $\delta$; Anthophor.: Anthemoessat of, Clisodon $q$, se.

Short-tongued Bees (17) - Maliet. (Q) : Agapustemon virilulus se, ab, Augochlora fervida e, C'hloralietus pilosus se. sparsus se. versatus se, ah, zephyrus, Curtisapis eoriacea e. Evelarus portoralis, Halietus parallelus se, Odontalietus, Oxystoglossa similis se, fq. Seladonia se: Prosopid. (ㅇ. f, n) : Prosopis lyomam, ziziate: Andren.: Andrena geranii 9 , se. Trachandrena eratargi \&; Ponurg.: Calliopsis andreniformis $\delta$.

Other Hymenoptera (4)-.Sphec.: Priononyx atrata, Sphes nigricans, pietipennis, procera ab.

Diptera (13)-Syrph.: Eristalis dimiliatns, flavijus, transversus fq. Melophilus latifroms. Voluenlla eveta 1 ; Fmpid.: Parempis; Jombyli.: Anthrax parvicomis. Bombylius atriceps ab, Areyramoeba oedipus ?. n, Toxophora; Conop.: Physoeephala tihialis; Tachin.: Archytas aterrima, Epigrimyia polita.

Lepiloptera (1.5)-Nymphal.: Anosia. Areymnis, Charidryas; Pupilion.: Eurymus, Iphiclides, Jasoniades, Pieris, l'ontia ; Hesperi: Achalarus, Epareyreus. Euphyns verua, Limonores manafaaqua, taumas, Pholionra hayhurutii, Therybes pylarlis. 
Coleoptera (2, sf, n)—Scarabae.: Trichius affinis, piger fq.

Blephilia hirsuta (14:114-5).-Blooms June 21-September 3; 83 visitors observed, June 26-July 29 ; Mas, W.

Long-tongued Bees (36)-Ap.: Apis ab; Bomb.: Bombias auricomus $q$, separatus $\delta^{\lambda} \not, f q$, Bombus americanorum $\delta^{\lambda} q \not{q}, \mathrm{fq}$, impatiens $\delta$, ridingsii $\delta$, fq, Psithyrus laboriosus $q$, variabilis $q$; Ceratin.: Ceratina $q$, sc, ab, Zaodontomerus $\delta^{\star}$; Megachil.: Trypetini : Neotrypetes truncatus $q$, sc; Osmiini : Alcidamea simplex $q$; Megachilini : Chelostomoides $\delta^{\lambda}+, f q$, Megachile brevis $\delta^{\lambda} q$, generosa

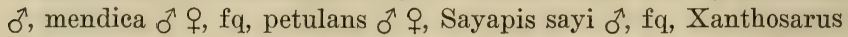
$\delta^{\pi}$; Coelioxyini : Coelioxys 8-dentata $\delta^{\pi}$, rufitarsis $\delta^{\pi}$, sayi $o^{\pi} q, \mathrm{fq}$; Epeol.: Triepeolus concavus ô, concolor ơ , fq, cressonii ô, remigatus + ; Eucer.: Epimelissodes obliqua $0^{\top}+$, Florilegus $q$, Melissodes

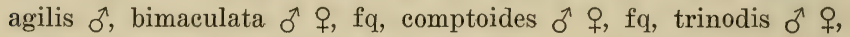
variabilis $\sigma^{\pi} q$, Peponapis $\delta$; Anthophor.: Amegilla $q$, Clisodon co $q$, se.

Short-tongued Bees (13)-Halict.: Agapostemon radiatus $\delta^{\lambda}$, se, $f q$, splendens $\sigma^{\gamma}$, viridulus + , Augochlora viridula $\delta^{\lambda}$, Chloralictus pilosus $q$, sparsus e, ab, n, Evylaeus areuatus $\delta^{\lambda}$, pectoralis + , se, 4-maculatus $q$, c, n, Halictus lerouxii $\delta$ 으. Oxystoglossa confusa $\delta^{1}+$, se, ab, pura $q$; Prosopid.: Prosopis ziziae $q, \mathbf{f}, \mathrm{n}$.

Other Hymenoptera (6) -Sphec.: Sphex nigricans, procera, vulgaris; Scoli.: Elis 5-cineta; Eumen.: Leionotus foraminatus fq; Pompil.: Ceropales fulvipes.

Diptera (16)-Syrph.: Allograpta f, n, Eristalis transversus, Syritta $f$, fq, n, Tropidia albistylum, Xylota fraudulosa $f, n$; Empid.: Parempis; Bombyli.: Anthrax parvicornis, sinuosa f, $\mathrm{n}$, Argyramoeba albofasciata $\mathrm{f}, \mathrm{n}$; Conop.: Oncomyia, Physocephala texana fq, tibialis, Stylogaster neglecta ab, Zodion obliquefasciatum; Tachin.: Archytas analis; Musc.: Stomoxys.

Lepidoptera (12)-Nymphal.: Anosia, Argynnis, Charidryas, Vanessa atalanta, cardui; Papilion.: Eurymus fq, Pieris, Pontia; Hesperi.: Epargyreus, Euphyes metacomet, Polites; Ctenuch.: Scepsis.

Hedeoma pulegioides $(14: 108)$.-Blooms July 21-September $28 ; 8$ visitors observed, August 8,$11 ; \mathrm{Mi}, \mathrm{R}$.

Long-tongued Bees (1) Ceratin.: Ceratina 우.

Short-tongued Bees (6)-Halict.: Chloralictus versatus $q$, sc, tegularis $q$, se, Odontalictus $\sigma^{\lambda}$, Oxystoglossa confusa ${ }_{+}$, similis $\sigma^{\lambda}$ 우, se, fq; Panurg.: Calliopsis andreniformis $\sigma^{\lambda} q, a b$.

Diptera (1)—Syrph.: Mesogramma marginata $\mathrm{f}, \mathrm{n}$. 
Isanthus brachiatus.-Bleoms August 19-Oetober 20; 8 visitors observed, August $29 ; \mathrm{Mi}, \mathrm{R}$.

Short-tongued Bees (3)-Mulict. (우. c) : Chloralictus pilusus, sparsus, versatus.

Leonurus cardiaca (int, 14:123).-Blooms June 4-Uetoluer 1s; 11 visitors observed, June 15-July 1; Ma, R.

Long-tongued Bees (5) - Ap.: Apis ab; Bomb. 185: Bombias aurieomus, Bombus amerimanom; Corutin.: Ceratina o. se, fy; Anthophor:: Anthemoessa of.

Short-tongued Bees (3)-IIalict. (f, c, n): Chloralictus sparsus, versatus ab, Seladonia.

Diptera (3)-Syrph. (f, n) : Allograptil, Mesogranma geminata, Syrphus ribesii.

Lycopus americanus $(14: 103-4)$.-Bloms July $15-$ September 16 ; 140) visitors observed, August 13-September 7; Pol, W.

Long-tongued Bees (13)-Ap.: Apis ab; Bomh.: Bombus

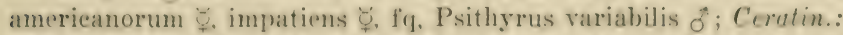
Ceratina $q$, se, fq; Megachil.: Megachilini: Merachile brevis $q$, mendica ô, petulans ô; Coelioxyini : Coelioxys germana $\$$; Epeol.: Triepeolus eoneolor $q$; Eucer.: Mrelissodes emptoides of $q$. nivea $q$, simillima $\delta$.

Short-tongued Bees (17)-Halict.: Agapostemon radiatus of $q$, Augochlora fervida $\sigma^{+} q$, fq, Chloralictus sparsus $q$, se, versatus $q$, se, ab, Curtisapis coriacea $q$, Halietus lerouxii $\delta$, parallelus of, Odontalietus $q$, Oxystoglossa similis $q$, Seladonia of $q$; Prosupid.: Prosopis illinoensis $q$. pygmaea of, ziziae ô $q$; Collet.: Colletes americanus of. latitarsis of; Panurg.: Calliopsis andreniformis $f$. se, Perdita $\delta^{\top}$ ㅇ, in cop.

Other Hymenoptera (36)-s phec: Ammobia ichneumnea fo. pennsylvanica, Isodontia apiealis, Priononyx atrata, thomae, Sceliphron, Sphex graeilis, nigricans ab, pictipennis, procera: Bumbic.: Bicyrtes ventralis, Megastizus fq, Stizus; Scoli.: Elis interrupta fq, 5-cincta fq. Seolia bicineta; Philanth.: Enereeris fq, Philanthus punctatus, ventilabris; Tesp.: Polistes annularis, pallipes, variatus, Vespula maculata; Eumen.: Eumenes fraternus. Leionotns foraminatus, vagus; Larr.: Tachytes aurulentus, elongafus, mandibularis; Pompil.: Ceropales bipunctata, Lophopompilus philadelphieus, Poecilopompilus interruptus, navus, Pompiloides marginatus, Prioen.moides fulvicornis fq: Chrysid.: Heilychrum wiltii.

Diptera (35) - Syrph.: Mesogramma marginata, Orthoneura nitida, Paragus bicolor, tibialis, Sphaerophoria, Syritta ; Empid.: 
Parempis; Bombyli.: Exoprosopa fasciata, fascipennis; Conop.: Conops xanthopareus, Physocephala texana; Tachin.: Archytas analis ab, aterrima ab, hystrix, Cistogaster pallasii, Cyphocera, Distichona auriceps, Gymnosoma, Linnaemyia, Ocyptera lata, tenuis, Peleteria $\mathrm{fq}$, Senotainia rubriventris, Siphoplagia anomala fq, Spallanzania ab, Trichopoda pennipes, Trichophora; Dexi.: Scotiptera; Sarcophag.: Helicobia helicis, Scarcophaga sinuata; Musc.: Compsomyia, Lucilia sericata, sylvarum, Pseudopyrellia; Anthomy.: Limnophora.

Lepidoptera (8)-Nymphal.: Basilarchia archippus, Phyciodes; Lycaen.: Everes; Papilion.: Eurymus; Hesperi.: Ancyloxypha, Limochores taumas, Pholisora catullus; Ctenuch.: Scepsis.

Coleoptera (1) -Lampyr.: Chauliognathus pennsylvanicus ab. On August 22, 24, 27, 29, 1910, the following 51 species and 383 individuals were taken on the flowers:

Long-tongued Bees (3:13)-Ap.: Apis 10; Megachil.: Megachile brevis 2 ; Eucer.: Melissodes comptoides 1.

Short-tongued Bees (3:11)-Halict: Augochlora fervida 1, Chloralictus sparsus 1, versatus 9 .

Other Hymenoptera (17:51) —Sphec.: Ammobia ichneumonea 2 , Priononyx atrata 3 , thomae 2, Sceliphron 1 , Sphex nigricans 9 ; Bembic.: Bicyrtes ventralis 1, Megastizus 6, Stizus 1; Scoli.: Elis 5-cincta 6; Philanth.: Eucerceris 2; Vesp.: Polistes pallipes 3; Larr.: Tachytes aurulentus 1, elongatus 2, mandibularis 1; Pompil.: Lophopompilus philadelphicus 1, Poecilopompilus interruptus 2, Priocnemoides fulvicornis 8 .

Diptera (24:141)—Syrph.: Mesogramma marginata 1, Syritta 2 ; Bombyli.: Exoprosopa fasciata 2; Conop.: Conops xanthopareus 1; Tachin.: Archytas analis 24, aterrima 5, hystrix 1, Cistogaster pallasii 1, Cyphocera 2, Gymnosoma 3, Linnaemyia 1, Ocyptera tenuis 2, Peleteria 5, Senotainia rubriventris 5, Siphoplagia anomala 8, Spallanzania 64, Trichophora 4; Sarcophag.: Helicobia helicis 2, Sarcophaga sinuata 4; Musc.: Compsomyia 1, Lucilia sericata 1, sylvarum 3, Pseudopyrellia 2; Anthomy.: Limnophora 1.

Lepidoptera (3:5)-Nymphal.: Basilarchia archippus 1, Phyciodes 3 ; Hesperi.: Limochores taumas 1.

Coleoptera (1:162)—Lampyr.: Chauliognathus pennsylvanicus 162.

Marrubium vulgare (int, 14:122-3).-Blooms May 21-October 5; 7 visitors observed, June 22, 25, 29, September 9; Ma, W.

Long-tongued Bees (5)-Ap.: Apis ab;Bomb. (ఫ) : Bombias separatus, Bombus americanorum; Ceratin.: Ceratina $q$; Megachil.: Megachile brevis $q$. 
Long-tongued Bees (21) - Ap.: Apis, at holes made by Leionotus, ab, n; Bomb.: Bombias auricomus $\hat{\sigma} q \not{q}, \mathrm{ab}$, separatus $\delta^{\lambda}$

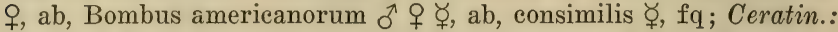
Ceratina $\rho$, e and at holes made by Leionotus, fq, n; Megachil.: Megachilini : Cyphopyga ô, Xanthosarus $q$; Coelioxyini : Coelioxys rufitarsis $q$; Epeol.: Triepeolus concavus $q$, concolor, $q$, nevadensis $\delta$, remigatus $q$; Eucer.: Anthedon $\delta$, ab, Epimelissodes obliqua

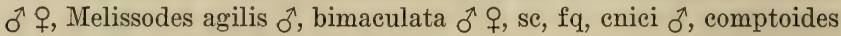
$\tau+$, trinodis $q$, variabilis $q$, se, type.

Short-tongued Bees ( 7 , + , at holes made by Leionotus) Halict.: Agapostemon radiatus, Chloralictus pilosus, versatus, also c, Halictus lerouxii, Odontalictus, Oxystoglossa confusa, Seladonia.

Other Hymenoptera $(3, \mathrm{n})$-Sphec.: Sphex pictipennis, at holes; Eumen.: Leionotus dorsalis, perforating, foraminatus, perforating.

Diptera (6)-Bombyli.: Exoprosopa decora, fasciata ab, Argyramoeba albofasciata f, n, Systoechus fq; Conop.: Physocephala tibialis, Stylogaster biannulata n.

Lepidoptera (23)-Nymphal.: Anosia, Argynnis, Basilarchia archippus, Speyeria, Vanessa atalanta, cardui, huntera; Papilion.: Euphoeades, Eurymus, Heraclides, Laertias ab, Papilio ab, Pontia; Hesperi.: Achalarus, Atrytone delaware, zabulon, Epargyreus, Euphyes metacomet, Pholisora catullus, hayhurstii, Thorybes bathyllus; Sphing.: Hemaris axillaris, thysbe.

Nepeta cataria (int, 14:116-7).-Blooms June 19-November 19; 73 visitors observed, June 26-September 10 ; Mas, R.

Long-tongued Bees (28)-Ap.: Apis ab; Bomb.: Bombias

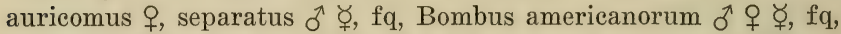
consimilis $\not{\phi}, \mathrm{se}, \mathrm{fq}$, impatiens $\delta^{\lambda} \not{\psi}, \mathrm{fq}$, ridingsii $\sigma^{\lambda}$; Ceratin.: Ceratina + ; Megachil.: Trypetini: Neotrypetes truneatus $q$; Osmiini: Alcidamea simplex $q$; Megachilini : Anthemois $\sigma^{\top} q$, Chelostomoides

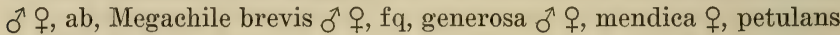
$\uparrow$, Oligotropus $\delta^{\lambda}$, Sayapis pugnata $\delta^{\lambda}$; Coelioxyini: Coelioxys $8-$ dentata $\sigma^{\lambda}$, sayi $\sigma^{\lambda}+$; Nomad.: Centrias americanus $q$; Epeol. $\left({ }^{\Uparrow}\right)$ : Epeolus bifasciatus, Triepeolus concavus, concolor fq, remigatus; Eucer.: Epimelissodes obliqua $\hat{\sigma}$, Melissodes bimaculata $\hat{0}+\mathrm{fq}$, variabilis ${ }^{\lambda}$.

Short-tongued Bees (10)-Halict.: Agapostemon radiatus ô $q$, fq, viridulus $q$, Chloralictus pilosus $\delta$, sparsus $q$, c, versatus $q$, sc, fq, Halictus lerouxii $\hat{\sigma}+$, fq, parallelus $q$, Odontalictus $q$, Seladonia ô $q$; Panurg.: Calliopsis andreniformis $q$.

Other Hymenoptera (11)—Sphec.: Priononyx atrata, Sphex gracilis, nigricans, pictipennis, procera, vulgaris; Scoli.: Elis 5cincta; Eumen.: Leionotus anormis, dorsalis, foraminatus, fulvipes. 
Diptera (12) -Symph.: Eristalis tenax, Ielophilus latifrons, Rhingia, Sphacrophoria, Syritta; Bombyli.: Anthrax fulwohirta, parvieornis; Tuchin.: Arehytas analis, aterima, Cyphocera, Linnaemyia, Ocyptera lata.

Lepidoptera (11) - Nymphal.: Argrnnis, Pliyeiodes, Vanessat

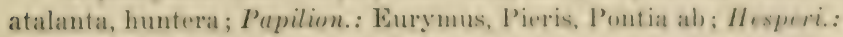
Epargyreus, Pholisora cafullus, Thanatos jurenalis, martialis.

Hemiptera (1) - Lygae.: Lygaeus.

Nepeta hederacea (int, 14:117-8).-Blooms Mareh 2f-Jume 21 ; 22 visitors observed, Mareh 25-May 20; Ma, R.

Long-tongued Bees (12)-Ap.: Apis ab; Bomb. (?): Bombus americanorum ab, eonsimilis, penusylvaniens, ridingsii; Cerutin. Ceratina $\delta{ }^{\circ}$, ab; Megachil.: Alcidamea simplex $\delta$, Osmia collinsiam

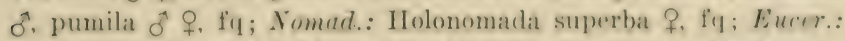
Tetralonia dileeta of $q$, ab; Anthophor.: Anthemoessa of $q$, ab.

Short-tongued Bees (2, \&)-IIulict.: Augochlora viridula, Oxystoglossa confusa.

Diptera (2)—Syrph.: Rhingia ; Bombyli.: Bombylius atriceps ab.

Lepidoptera (6. n)-P'apilion.: Eurymus ab, Pieris; IIesperi.: Atrytone zabulon fq. Pamphila hobomok, Polites; Noctu.: Plusia simplex.

Physostegia virginiana (14:121-2).-Blooms July 1!)-October $10 ; 7$ visitors observed, Aurust 23 -September $22 ; \mathrm{Ma}, \mathrm{R}$.

Birds (1)-Trochil.: Trochilus 2.

Long-tongued Bees (4)-Bomb.: Bombus americanorum of $q$ \% 20. consimilis है. sc, 1; Megachil.: Megachile brevis d. 1 ; Euct.: Melissodes bimaculata $\delta$, 1 .

Lepidoptera $(2, n)-$ Tymphal.: Anosia 1; Papilion.: Eurymus 1.

Prunella vulgaris $(14: 120-1)$.-Bloums July 6-November 19: 23 visitors observed, July 11-Oetober 3 ; Ma, R.

Long-tongued Bees (9)-Bomb.: Bombias separatus d. Dombus americanorum $8, a b$, consimilis 8 impatiens $d 8$, se, fq; Ciretin.: Ceratina f; Meguchil.: Megachile mendiea of; Eucr.: Meliseodes

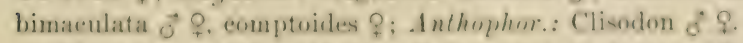

Shurt-tongued Bees (3, \%)-IInlict.: Oxystoglusa confusa, Sieladonia n; Pamurg.: Calliopsis andreniformis.

Other IIymenoptera (1)-Scoli.: Scolia bicincta.

Diptera (2)-Bombyli.: Exoprosopa fasciata; Tuchin.: Arehytas aterrima. 
Lepidoptera (8, n)-Papilion.: Eurymus, Pieris, Pontia; Hesperi.: Ancyloxypha, Atrytone delaware, Limochores taumas, Pholisora hayhurstii, Polites.

Pycnanthemum flexuosum (14: 107).-Blooms June 19-September $12 ; 268$ visitors observed, June 19-August 3; Mis, W.

Long-tongued Bees (44)-Ap.: Apis ab; Bomb.: Bombias auri-

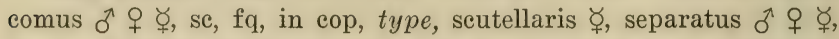
se, fq, Bombus americanorum ô $q \not{q}$, impatiens $q$, $\not$, Psithyrus laboriosus $q$, variabilis $q$; Ceratin.: Ceratina $q$; Stelid.: Stelidium O, type; Megachil.: Osmiini: Alcidamea simplex $q$; Megachilini: Megachile brevis $\delta q, \mathrm{fq}$, generosa $\delta^{\lambda} q$, mendica $\delta q$, ab, petulans

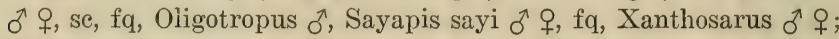
Coelioxyini : Coelioxys alternata $\delta$, germana $\delta q$, modesta $\delta$, 8-den-

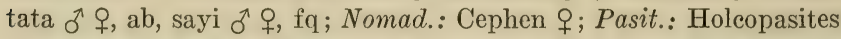
illinoensis $\sigma^{7}+$; Epeol.: Epeolus bifaseiatus $\sigma^{\lambda}$, fq, lectoides + , type, Triepeolus concavus $\delta^{\lambda}+, \mathrm{fq}$, concolor $\delta^{\lambda}+$, ab, cressonii $\delta^{\lambda}$, lunatus $\delta^{\lambda} q, \mathrm{fq}$, nevadensis $\delta^{\lambda}+$, remigatus $\delta^{\lambda}+\mathrm{fq}$, simplex $\delta^{\lambda}$; Melect.: Melecta $\delta$; Eucer.: Anthedon $\delta^{\tau} q$, Epimelissodes illinoensis $q$, obliqua $\delta \tau$, fq, Florilegus $q$, Melissodes agilis $\delta \tau$, bimaculata $\delta$, ab, comptoides $\delta$, cereopsis $q$, variabilis $\delta$.

Short-tongued Bees (38)-Halict.: Agapostemon radiatus $0^{\pi}$, $\mathrm{ab}$, texanus $q$, Augochlora fervida $\delta^{\lambda}+$, se, viridula $\sigma^{\lambda}+$, Chloralictus pilosus $\sigma^{\lambda}$, pruinosus $\delta^{\lambda}$, sparsus $q$, tegularis $q$, se, versatus $\delta^{\lambda} q$, se, fq, Curtisapis forbesii $\delta^{\pi} q, f q$, Evylaeus areuatus $\delta$, pectoralis $\delta^{\lambda} q$, se, Halictus lerouxii $\delta^{\lambda} q$, ab, parallelus $\hat{\delta} q, f q$, Odontalictus

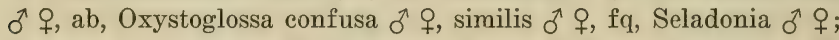
Sphecodini: Dialonia + , Machaeris stygia $0^{\lambda}+{ }^{\circ}, a b$, Proteraner $\delta^{\lambda}$, Sphecodes arvensis $\delta \hat{q}$, ab, heraclei $\delta$, minor $q$, Sphecodium cressonii $q$, pyenanthemi $q$, type; Prosopid.: Prosopis pygmaea $q, \mathrm{fq}$,

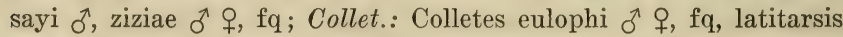

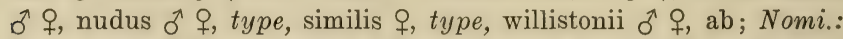
Paranomia of $q, \mathrm{ab} ;$ Andren.: Trachandrena obscura $q$, type, quintilis + , fq, type; Panurg.: Calliopis andreniformis $q$.

Other Hymenoptera (89)-Sphec.: Ammobia ichneumonea fq, nuda, pennsylvanica, Isodontia apicalis, Palmodes, Priononyx atrata $f q$, thomae, Sceliphron, Sphex gracilis in cop, nigricans $f q$, pictipennis, procera; Bembic.: Bembix nubillipennis $f q$, spinolae, Bicyrtes 4-fasciata fq, ventralis, Megastizus, Stictia, Stictiella fq, Stizus; Scoli.: Campsomeris, Elis interrupta, obscura ab, 5-cincta ab, thoracica, Scolia bicincta, nobilitata; Philanth.: Cerceris bicornuta, clypeata, compacta, compar, finitima, fumipennis, robertsonii, rufinoda, Eucerceris, Philanthus punctatus fq, ventilabris fq; 
Vesp.: Polistes annularis, rubiginosus, variatus; Lumen.: Ancistru. cerus campestris, capra, unifaseiatus, Fumenes fraternus in oop, Leionotus anomuis fị, arvensis, elypatus type, dorsalis, foraminatus ab, fulvipes, fundatus, histrionalis, molestus, scrophulariae,

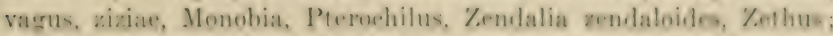

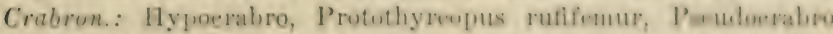
fq. Solenius ab: Ozubel.: Notoglowat americana, emarminata, frontalis. Oxybelus laetus, paekarilii; Larr.: Astanus unientor. Noto. gonidea, Tarhysphex arutus, lelfragei, munlus. Tarhytes aurulen. tus, elongatus ab. harpax, pepticus: Nyssom.: Pourloplisus phaleratus; l'ompil.: Arachnoproctonus, Batimuns. Cemplese rohinsonii, Loplopompilus philadelphieus, Poncilopompilus anvus. Pompiloides americanus, marrinatus, Priocnemojdes fulvicomis 67 ; Chrysid.: Hedyehrum violaceum.

Diptera (54)-Strutiomy.: Glontomyia eincta, Stratiomyia meigenii; Syrph.: Eristalis afneus, dimidiatus, latifrons fo, tomax fi], trausversus, vinetorum fq. Helophilas latifrons, Mallota rimbiciformis, illinoensis type, Mesogramma marrinata ah, Milesia, Orthoneura nitida iq. Sphaerophoria, Spilomyia longiromis, Syritta ab, Tropidia mamillata; Empid.: Parempis ab; Midu.: Mirlas elavatus ab, tibialis: Bombyli.: Anthrax alternata, fulvolirta lq. parvicornis. Argyramomba albofasciata i, n, Exoprosupa emarginata fq, fasciata, fasr.iprnnis ab: (onop.: Conops brachyrryorhus. santhopareus ab, in cop. Oncomyia. Physocophala texana, tibialis ab, Zodion fulvifrons, obliqueiaseiatum in eop; Tarhin.: Alophomacnenventris ah, fumosa, Arehytas analis ab, aterrima, (istoguster oveidua, pullasii iq. Clytionyia. Epierimyia polita, Linnarmyia. Ocyptera lata. teunis, Paradidyma, Phorantha humeralis, Spallanzania. Xantho. melana: Masc.: Compromyia, Phormia terracnovar, P-udopyrellia : Anthomy.: Limnophora.

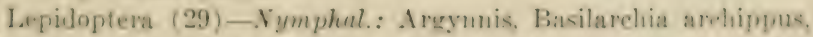
Charidryas, Euptoieta, Hypatus. Jumonia, Nenympha, I'hyeoides. Tanesea atalanfa, cardui, huntera ab; Lycen.: Thrysphanus, Everes, Strymon, Uvanotes; Papilion.: Eurema. Eurymus. Iaertias. Pieris, Pontia, Zerrene: Hesperi.: Epargyreus, Limonoms tamman, Pholisura eatullus, Polites. Thanaos juvenalis, martiulis. Thorylors bathyllus; Ctonuch.: Seepsis.

Coleoptera (9)-Gerambye.: Typoerus sinuatu iq : Curculium :

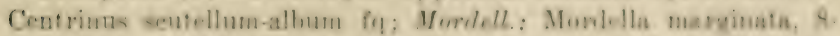
punetata: Rhipilhor.: Rhipiphorus dimidiatus. Alaviponnik, lim. batus ab; Scarabae.: Triehius affinis, piger.

Hemipters (5)-Caps.: Caloworis: Lysus: Lygaens, Oneopeltus fq, Ortholomus; Pcntatom.: Euchistus ietericus. 
Pycnanthemum pilosum (14:106).-Blooms July 5-September $8 ; 113$ visitors observed, July 21-August 29 ; Mis, W.

Long-tongued Bees (17)-Ap.: Apis ab; Bomb.: Bombus americanorum $\Varangle ;$; Cratin.: Ceratina $\$$; Megachil.: Megachilini : Megachile brevis $q$, mendica $\delta^{\lambda}$, petulans $\delta^{\lambda}$, fp, Xanthosarus $\delta^{\lambda}$; Coelioxyini $\left(\circlearrowleft^{\top}\right)$ : Coelioxys germana fq, 8-dentata, sayi; Epeol.: Epeolus bifasciatus $\delta^{\lambda} q, \mathrm{ab}$, Triepeolus concavus $\delta^{\lambda}+, \mathrm{fq}$, remigatus $q, \mathrm{fq}$, simplex $\sigma^{\star}$; Eucer.: Epimelissodes obliqua $\sigma^{\lambda}$, Melissodes agilis $\sigma^{2}$, comptoides $0^{\top}+$, ab.

Short-tongued Bees (16)-Halict.: Agapostemon radiatus ot, ab, Augochlora viridula $\delta^{\lambda} \uparrow, \mathrm{fq}$, Chloralictus pilosus $q$, sparsus $q$, sc, $a b$, versatus $\hat{\sigma} q$, se, ab, zephyrus $q$, Evylaeus areuatus $\hat{\sigma} q$, pectoralis $q$, Halictus lerouxii $\delta^{\lambda}+$, ab, parallelus $\delta^{\lambda} q, \mathrm{fq}$, Odontalictus $\delta^{\lambda}+$ ab, Seladonia $\delta^{\lambda}+, a b$; Sphecodini: Sphecodes arvensis $\delta^{\lambda}$ , fq, Sphecodium cressonii ot; Prosopid.: Prosopis ziziae $\$$; Panurg.: Calliopsis andreniformis $\delta^{\lambda}+$, sc, fq.

Other Hymenoptera (43)-Sphec.: Ammobia ichneumonea, nuda, Isodontia apicalis, Priononyx atrata, Sphex gracilis fq, nigrieans, pictipennis $f q$, procera, vulgaris; Bembic.: Bembix nubillipennis ab, Bicyrtes 4-fasciata, ventralis, Stictia, Stictiella; Scoli.: Elis interrupta, 5-cincta ab, Scolia bicincta; Philanth.: Cerceris compacta, frontata, fumipennis fq, Eucerceris, Philanthus punctatus $\mathrm{fq}$, ventilabris $\mathrm{fq}$; Vesp.: Polistes variatus; Eumen.: Leionotus anormis, arvensis, dorsalis, foraminatus ab, fundatus, histrionalis, molestus $\mathrm{f} q$, scrophulariae, vagus $\mathrm{f} q$, Pterochilus, Zendalia zendaloides type; Crabron.: Anacrabro, Solenius; Oxybel.: Notoglossa frontalis; Larr.: Tachytes aurulentus, elongatus, mandibularis; Pompil.: Priocnemoides fulvicornis; Chrysid.: Hedychrum wiltii.

Diptera (31)-Stratiomy.: Stratiomyia meigenii; Syrph.: Eristalis dimidiatus, tenax, transversus, Syritta ab; Bombyli.: Anthrax fulvohirta, Exoprosopa emarginata, fascipennis ab, Geron rufipes, Systoechus; Conop.: Conops xanthopareus fq, Oncomyia, Physocephala tibialis, Zodion fulvifrons, obliquefasciatum; Tachin.: Archytas analis ab, aterrima, Cistogaster occidua, pallasii, Ocyptera tenuis, Phorantha magna, purpurascens, Senotainia trilineata, Siphona, Spallanzania, Trichopoda pennipes, Trichophora; Sarcophag.: Helicobia helicis; Musc.: Compsomyia, Phormia regina, Stomoxys.

Lepidoptera (4)-Nymphal.: Phyciodes; Papilion.: Pontia; Hesperi.: Epargyreus, Thanaos martialis.

Coleoptera (2)-Rhipiphor.: Rhipiphorus dimidiatus in cop, limbatus. 
Pycnanthemum virginianum (14:104-6).-Bloums July 12September 7 ; 66 visitors observed, July 12-Septrmber 6 ; Mis, W.

Long-tongued Bees $(10)-1$ p.: Apis ab; Bomb.: Bombias scutellaris $\Varangle$. separatus $q$; Meguchil.: Neotrypetes truncatus of Coelioxys S-dentata $q$; Epeol.: Epeolus bifasciatus of Trippeolus coneolor $\sigma^{*}$, fy; Eucer.: Epimelissodes atripes f. Florilegus $\delta$, Melissodes comptoides $\sigma^{\wedge}$.

Short-tongued Boes (15)-Hulict.: Agapostemon radiatus d,

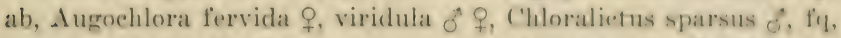
versatus of $o^{+}$ab, Evylaeus arouatus of, pectoralis $q$. Haliotus lerouxii of q. parallelus f. Odontalietus f, Seladonia of f, ab; Sphecodini: Drepanium $q$. Spheredes arvensis of $q$, ab; Nomi.: Paranomia ôे Panurg.: Calliopsis andreniformis of $q$.

Other Hymenoptera (23) - Sphec.: Ammobia nnda, mennsylvaniea, Sphex gracilis, nierricans, pietipennis, proeera ab, vulgaris; Bembie.: Bicyrtes t-fasciata, Menastizus, Stietiella; Senli.: Elis interrupta, J-cincta, Scolia bicincta; Philunth.: Cereris fumipennis, Eucereeris fq; Eumen.: Emmenes fraternus, Leionotus arvensis, dorsalis, foraminatus fq, Zethus; Oxybel.: Notoglossia americana; Larr.: Tachytes aurulentus, elongatus.

Diptera (14) $-S y r p h .:$ Eristalis tenax, Sphaerophoria, Syritta ; Bombyli.: Exoprosopa fasciata, fascipennis ab. Systropus; ('onop).: Oncomyia, Zodion fulvifrons; Tachin.: Alophoral aeneoventris, Arehytas analis ab, Cistogaster oceidna, Spallanzania fq, Triehopoda pennipes; Musc:: Compsomyia fq.

Lepidoptera (2) - Tymphal.: Phyciodes fy: I'apilion.: Pontial.

Coleoptera (1)-Rhipiphor.: Rhipiphorus limbatus.

Hemiptera (1)-Lygae.: Lygaeus.

Scutellaria canescans $(14: 119-20)$.-Blooms July 7-Siejtember 19 ; 13 visitors observed, July 31-August 26; Ma, R.

Long-tongued Beys (6)-Bomb.: Bombias aurieomus by, sepraratus 7 . Bombus americanorum of $b$, se, ah, consimilis $\phi$, se, impatiens $\not$, se; Ceratin.: Ceratina $q, \mathrm{fq}, \mathrm{n}$.

Short-tonerued Bees (:3)-Malict. (9. surking at holes male hy Leionotusi : Chloralictus sparsus, versatus, Osystuglosia eunfusa.

Gther Hymenoptera (1) -Eumen.: Leionotus foraminatus. porforating.

Diptera (1)-Bombyli.: Exoprospa faseiata n.

Lepirloptera (2., и)-Mesperi.: Atrytune vahulon, Epartyrens.

Scutellaria laterifora.-Blooms July $18-$ S.pltemher 28,1 visifur observed, July 20; Ma, R. 
Long-tongued Bees (1)-Eucer.: Melissodes bimaculata ․

Scutellaria parvula $(14: 118-9)$.-Blooms May 8-June $29 ; 16$ visitors observed, May 19-June 3; Ma, R.

Long-tongued Bees (6)-Bomb.: Bombus americanorum $q$; Ceratin.: Ceratina $\delta^{\lambda}+$, ab, Megachil.: Osmiini: Alcidamea simplex ${ }^{\pi}$ ㅇ, sc, ab, Diceratosmia + , Osmia pumila $q$ se; Megachilini : Megachile brevis 0 .

Short-tongued Bees (5)-Halict. ( + ): Chloralictus pilosus, pruinosus fq, type, tegularis fq, Evylaeus pectoralis, Odontalictus.

Diptera (2, f, n)—Syrph.: Sphaerophoria, Syritta.

Lepidoptera $(3, \mathrm{n})$-Hesperi.: Ancyloxypha, Pholisora catullus fq, Polites.

Scutellaria versicolor $(14: 120)$.-Blooms June 7-July $26 ; 10$ visitors observed, June 14-July 10 ; Ma, R.

Long-tongued Bees (7)-Bomb. ( $q$ q) : Bombus americanorum, consimilis se, fq; Ceratin.: Ceratina $q$, se, n; Eucer.: Tetralonia dilecta $q$; Anthophor.: Anthemoessa $\sigma^{\top} q$, se, fq, Anthophora ㅇ, se, Clisodon $q$, sc.

Diptera (1)—Syrph.: Rhingia $\mathrm{f}, \mathrm{n}$.

Lepidoptera $(2, \mathrm{n})$-Papilion.: Euphoeades, Papilio.

Stachys palustris (14:124-5).-Blooms June 2-September 28; 21 visitors observed, June 2-September $10 ; \mathrm{Ma}, \mathrm{R}$.

Long-tongued Bees (10)-Bomb.: Bombias auricomus $\Varangle$, Bombus americanorum $\delta \bar{\gamma}, \mathrm{fq}$, consimilis $\phi$, impatiens $\Varangle$; Ceratin.: Ceratina + , ab; Megachil.: Alcidamea simplex $q$, sc, fq, Megachile brevis + , ab ; Nomad.: Holonomada superba $q$; Eucer.: Melissodes bimaculata $\widehat{0}+, \mathrm{fq}$; Anthophor.: Anthemoessa 0 .

Short-tongued Bees (3)-Halict. () : Agapostemon viridulus, Chloralictus versatus e, $\mathrm{n}$; Panurg.: Calliopsis andreniformis 07 .

Diptera (3)-Syrph. (f, n) : Mesogramma marginata, Syrphus ribesii; Bombyli.: Systoechus.

Lepidoptera (5, $\mathrm{n})$-Papilion.: Iphiclides; Hesperi.: Ancyloxypha, Limochores taumas; Noctu.: Plusia precationis, simplex.

Teucrium canadense (14:101-2).-Blooms June 19-September $7 ; 45$ visitors observed, June 30 -August 19 ; Mas (Ma), R.

Birds (1) - Trochil.: Trochilus.

Long-tongued Bees (28)-Ap.: Apis ab; Bomb.: Bombias scutellaris $\not$, separatus $\delta^{\lambda} q \not{q}, \mathrm{fq}$, Bombus americanorum $\delta^{\lambda} \not, \mathrm{sc}, \mathrm{fq}$, consimilis $\not{q}$, impatiens $\not{\psi}, \mathrm{f} q$, Psithyrus variabilis $q$; Ceratin.: Ceratina + , fq, n; Megachil.: Osmiini: Alcidamea simplex $q$; 
Megachilini: Megachile brevis of $q$, se, fq, mendica + , Sayapis sayi of $q$. Xanthesarus of $q$; Coelinxyini : Coelioxys germana $q$; Epeol: Triepeolus concavus of, eoneolor of ${ }^{\circ}$. fq, simplex of: Eucer.: Epimelissodes obliqua of $q$, Florilegus of $f, \mathrm{se}, \mathrm{fq}$, Melissodes aquilis $d$, bimaculata of $q$, ab, eomptoides of, fq, petalostemonis of, trinorlis ? variabilis $\delta^{*}$, vernoniana $o^{*} q$ : Anthophor.: Amegilla $\delta$. Anthemoessa 우.

Short-tongued Bees (4)-Malict.: Agapostemon viridulus ? Halictus lerouxii of \&. Oxystoglossa confusa o, n. pura f, n.

Other Hymenoptera (1)-Bembic.: Bembix nubillipennis.

Diptera (5)-Bombyli.: Exoprosopa faseiata, fasipennis, Systoechus; Conop.: Physoeephala texana, Stylogaster biannulata.

Lepidoptera (6)-Lycaen.: Everes; Pupilion.: Eurymus, Laertias; Hesperi.: Atrytone delaware, Polites; Sphing.: Hemaris axillaris.

\section{Lauraceae}

Sassafras variifolium $(9: 164-5)$.-Blooms April 27-May 4; 91 visitors observed, April 27-May 4; Pol, Y.

Long-tongued Bees (1)-Megrachil.: Osmia pumila ?.

Short-tongued Bees (16)-IIalict. (q): Chloralictus eressonii, illinoensis, sparsus se, fq, versatus se, zephyrus se, Evylaeus areuatus, Halietus lerouxii; Spheeodini: Sphecodes arvensis, minor type: Andren: Andrena carlini $f$. salictaria $\delta$. Parandrena $\delta$, Pterandrena lauracea + , type, Trachandrena elaytoniae $\delta$, $\mathrm{fq}$. forbesii $q$, hippotes $\delta$.

Other Hymenoptera (12)-Vesp.: Polistes pallipes, variatus; Pompil.: Cryptocheilus conicus; Ichneumon.: Gilypta rufiseutellaris, Idiolespa, Ophion, Pimpla annulipes; Tenthredin.: Dolerus bieolor, חylotoma maeleayi, Macrophya epinota, Monophadnus, Opisthoneura.

Diptera (57)-Bibion.: Bibio femoratns in. pallipes iq simuli.: Simulium; Stratiomy.: Sargus; Syrph.: Brachypalpus frontosus, Chilosia capillata, punetulata fq. Chrysogaster outario, Criorhina umbratilis fq. Eristalis dimidiatus, transversus, Ielophilus similis, Melanostoma obseurum, Mesogramma marginata, Myiolepta strigil. ata, Orthoneura nitida, Platyehirus hyperboreus, quadratus, Psilota, Spharrophoria, Syrphus auerieanus, rib-sii, torsus: Empid.: Empis avila, compta type, otiosa. Rhamphomyia exigua, limhata, mutabilis fq. piligeronis type. priapulus fq, ravilla iq, in eup, type, sordila: Tarhin.: Euphorocera clariponnis, Gonia eapitata, I.innaemyia, Panzeria, Tachina robusta; Sarcophag,: Barttelievía. Cynomyia fq, Helieobia helicis, Sarenpluaga eimbieis. simuata: 
Musc.: Lucilia caesar, Morellia fq, Phormia regina, Pseudopyrellia; Anthomy.: Calythea, Coenosia antica, Homalomyia prostrata, Phorbia acra ab, platura ab; Agromyz.: Agromyza aeneoventris, latipes; Lonchae.: Lonchaea aberrans; Oscin.: Chlorops assimilis; Scatophag.: Scatophaga.

Lepidoptera (1) -Nymphal:: Vanessa huntera.

Coleoptera (3)-Coccinel.: Megilla; Lampyr.: Telephorus bilineatus fq; Oedemer.: Asclera puncticollis.

Hemiptera (1)_Corimelaen.: Corimelaena pulicaria.

LEGUMINOSAE

Amorpha canescens $(3: 80-1)$.-Blooms June 6-July 24; 49 visitors observed, June 12-28; Mis, R.

Long-tongued Bees (10)-Bomb.: Bombias separatus $\not$, Bombus americanorum $q \varnothing$, se, Psithyrus variabilis $q$; Ceratin.: Ceratina $q$; Megachil. (ㅇ) : Trypetini: Neotrypetes truncatus sc; Osmiini: Alcidamea simplex, Hoplitis se, fq; Megachilini: Megachile brevis se; Coelioxyini: Coelioxys 8-dentata, sayi.

Short-tongued Bees (15)-Halict.: Agapostemon texanus of, Chloralictus pilosus $q$, se, sparsus $q$, se, tegularis $q$, versatus $q$, se, fq, Curtisapis forbesii $\delta$, Halictus lerouxii $\sigma^{\lambda}+$, se, fq, parallelus $q$, Oxystoglossa confusa $q$, similis $\sigma^{\tau}+$, se, Seladonia $\sigma^{7}+$, se; Prosopid. $(, f, n)$ : Prosopis pygmaea, ziziae; Collet.: Colletes eulophi $\delta^{\lambda}$ 우, se, fq; Panurg.: Calliopsis andreniformis $q$.

Other Hymenoptera (16) -Sphec.: Isodontia apicalis, Priononyx atrata $\mathrm{fq}$, thomae, Sphex nigricans fq, pictipennis, procera, vulgaris; Bembic.: Bicyrtes ventralis; Scoli.: Elis 5-cincta; Philanth.: Cerceris bicornuta, Philanthus ventilabris; Eumen.: Eumenes fraternus, Leionotus anormis, dorsalis; Larr.: Tachytes elongatus, pepticus.

Diptera (5)-Syrph. (f, n): Paragus bicolor, Sphaerophoria, Tropidia mamillata; Bombyli.: Anthrax sinuosa f, n, Toxophora.

Coleoptera (3, f, n)-Chrysomel.: Diabrotica atripennis, 12punctata; Melo.: Macrobasis.

Amorpha fruticosa.-Blooms May 10-June 4; 9 species and 18 individuals taken on May 27; Mis (Mas), R.

Long-tongued Bees (3:10)-Ceratin.: Ceratina 9 , se, 8 ; Mega-

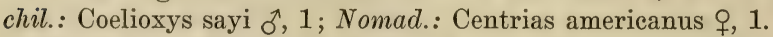

Short-tongued Bees ( $9,6: 8)$-Halict.: Chloralictus sparsus sc, 1, tegularis se, 1, Evylaeus pectoralis se, 3; Prosopid.: Prosopis saniculae 1 , sayi 1 , ziziae 1 . 
Amphicarpa pitcheri $(3: 200-1)$.-Blooms August 16-Sejtember $10 ; 4$ visitors observed, August $22-30 ; \mathrm{Ma}, \mathrm{R}$.

Birds (1)-Trochil.: 'Troehilus.

Long-tongued Bees (3)-Bomb.: Bombus amerieanorum of f, consimilis $\not{+}$, se, impatiens $\not{+}$, se.

Apios tuberosa.-Blooms July 3-August 26 ; 2 visiturs oberved, July 20, August 12; Ma, R.

Long-tongued Bees (2, f)-Megachil.: Megachile brevis se, mendica fq.

Astragalus canadensis (16:162).-Bloums July 3-Augu-t 26; 10 visitors observed, July 7-August 2; Ma, Y.

Long-tongued Bees $(7)-A p$.: Apis; Brmb.: Bombias auricomus $q \not$, se, iq, separatus $\phi$, Bombus americanorum $q \nabla$, se, i., Stelid.: Anthidium psoraleate of $q$, fq, in cop; Megachil.: Anthemois q; Eucer.: Melissodes bimaculata $\delta$.

Short-tongued Bees (1)-Hulict.: Halietus parallelus ?.

Other IIymenoptera (1)-Eumen.: Leionotus fundatus.

Lepidoptera (1)-Hesperi.: Thanaos martialis $\mathrm{n}$.

Astragalus mexicanus $(3: 199,16: 162)$.- Blooms April 15May 12 ; 16 visitors observed, April 19-May 4; Ma, Y.

Long-tongued Bees (11)-Bomb. (o) : Bombias aurieomus se, separatus, Bombus americanorum ab; Megnchil.: Osmia atriventris

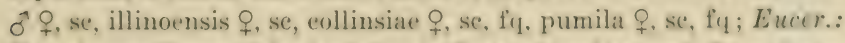
Tetralonia dilecta $\delta$, ab, dubitata $q, \mathrm{sc}, \mathrm{ab}$, robertsonii ${ }^{\circ}, \mathrm{se}$, type; Anthophor.: Anthophora of $q$, se, fq.

Diptera (1)-Bombyli.: Bombylius major.

Lepidoptera (4, n)-P'apilion.: Eurymus, Papilio; IIesperi.: Thanaos icelus, juvenalis.

Baptisia bracteata $(5: 176-7)$.-Bloms April $30-$ May $19 ; 4$ visitors observed, May 1-19; Ma, W.

Long-tongued Bees (4)-Bomb.: Bombias separafus o, Bombus amerieanorum ; Megachil.: Centrosmia of se; Eucer.: Tetralonia dilecta $\delta$ o $q$, fq.

Baptisia leucantha $(3: 79)$.- Blooms Jun 10-September 8 ; 2 visitors observed, July 11 and often; Ma, W.

Long-tongned Bees $(1,-B o m b$.: Bombus americanorum 7 . se, ab.

Lepidoptera (1)-Papilion.: Callidryas n.

Cassia chamaecrista $(3: 202-3)$. - Blooms Jume 28-0ctobur 5 ; 24 visitors observed, July 9-September 15 ; Ma, Y. 
Long-tongued Bees (18, c)-Ap.: Apis fq; Bomb.: Bombias auricomus $q \not$, ab, scutellaris $q \not$, separatus $\not, a b$, Bombus americanorum $\Varangle, a b$, consimilis $\Varangle$, impatiens $q \Varangle ;$ Megachil. ( $q)$ : Megachile brevis fq, c and cutting petals, generosa ab, mendica, petulans, Xanthosarus; Eucer.: (q), Epimelissodes atripes ab, oligolege, Florilegus, Melissodes bimaculata, comptoides ab, variabilis ; Anthophor.: Amegilla $q$, oligolege.

Short-tongued Bees (5,, , csp, $\mathrm{n})$ - Halict.: Agapostemon radiatus, texanus, Augochlora fervida, Chloralictus pilosus, versatus.

Diptera (1)—Syrph.: Milesia fsp, n.

Petiolar nectaries.-On sixteen days, June 29-August 26, the following 67 visitors were observed:-

Ants (4 ఫ)-Formic.: Camponotus castaneus Formica fusca, schaufussi fq, Lasius fq.

Short-tongued Bees (6)-Halict.: Chloralictus sparsus $\delta^{\lambda}$ 우 $a b$, tegularis $\delta^{\lambda}$, versatus $\delta^{\lambda}+, \mathrm{fq}$, zephyrus $\hat{\delta}$, Evylaeus pectoralis $\hat{\delta}$, Oxystoglossa similis 0 .

Other Hymenoptera (43)-Sphec.: Chalybion, Chlorion, Sceliphron; Philanth.: Cerceris kennicottii, rufinoda; Eumen.: Leionotus foraminatus; Crabron.: Lindenius robertsoni type, Rhopalum scutellatum; Oxybel.: Notoglossa americana, frontalis; Pemphredon.: Psen; Larr.: Astatus unicolor, Lyroda, Notogonidea, Tachysphex acutus, belfragei $f q$, mundus, tarsatus, Tachytes maestus, mergus, obscurus, sericatus; Nysson.: Nysson rufiventris, Zanysson aurinotus fq, plesia; Pompil.: Arachnoproctonus, Poecilopompilus navus, Prionenemis, Priocnemoides fulvicornis, terminatus, Psammochares pretiosa, relativus; Tiphi.: Tipha punctata, robertsoni; Mutill. $\left(\delta^{\top}\right)$ : Mutilla hexagona fq, sayi, Sphaerophthalma; Chrysid.: Chrysis intricata, texana, Tetrachrysis montana, Holopyga; Chalcid.: Perilampus cyaneus; Bracon.: Apanteles terminalis.

Diptera (13)—Taban.: Tabanus; Syrph.: Mesogramma marginata, polita, Syritta; Conop.: Oncomyia; Tachin.: Chaetoplagia, Exorista confinis, Senotainia rubriventris, trilineata ; Sarcophag.: Helicobia helicis; Musc.: Pseudopyrellia; Ortal.: Rivellia 4-fasciata; Oscin.: Chlorops assimilis.

Hemiptera (1)-Caps.: Lygus.

Cassia marilandica $(3: 203-4)$.-Blooms July 1-August 26 ; 3 visitors observed, July 21; Ma, Y.

Long-tongued Bees (1)-Bomb.: Bombus americanorum ఫ, c.

Short-tongued Bees (2,, , csp, $n)$-Halict.: Chloralictus sparsus, Oxystoglossa confusa. 
Petiolar nectaries.-Five visitors observed on July 21, 30.

Ants (4, ६)-Formic.: Camponotus hereulaneus 7 , Furmica schaufussi 1, Lasius 2; Myrmic.: Cremastogaster 3.

Coleoptera (1)-Coccinell.: Coceinella 9-notata 1.

Cercis canadensis $(3: 201-2)$.- Blooms Ipril + May 17; 52 visitors observed, April 21-May 5; Mi, R.

Long-tongued Bees (22)-Ap.: Apis se, ab; Bomk.: (f): Bombias aurienus ab, separatus se, Bombus amerieanorum, consimilis, impatiens; Ceratin.: Zaodontomerus of Meguchil. (se): Centrosmia of $q$, Ceratosmia of $f$, ah. Dieeratusmia $f$. Osmia atrivent ris $q$. pumila $q, f q ;$ Nomad.: Gnathias euneatus $\mathcal{d}$. Holonomada affabilis $\$$, Nomada denticulata $\delta$, sayi ${ }^{+}$. Xanthidium luteolvides

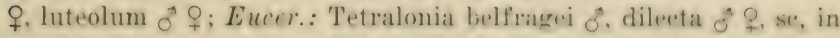
cop, ab) Anthophor.: Anthophora of o. fq. Emphoropsis $\delta$.

Short-tongued Bees (19)_IIulict. (q) : Agapostrmon radiatus, Augochlora viridula, Chloralietus pilosus, sparsus se, ab, versatus sc, fq, zephyrus, Curtisap is coriacea, forhesii, Evylaeus arcuatus, Inalictus lerouxii se, fq, Oxystoglossa eonfusa, pura, Seladonia se, ab): (collet.: Colletes inaequalis of $q$, se, ab) Andren.: Andrena car-

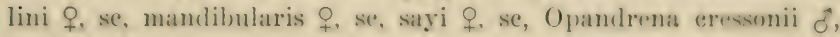
Trachandrena claytoniae $q$, se.

Other Hymenoptera (1)-Vesp.: Polistes variatus.

Diptera (4)-Empid.: Empis otiosa fq. Pachymeria, Rhamphomyia priapulus; Bombyli.: Bombylius major.

Lepidoptera $(5, \mathrm{n})$-Nymphal.: Vanessa atalanta, huntera; Lycaen.: Everes; Hesperi.: Thanaos icelus, juvenalis.

Coleoptera (1)—Cerambyc.: Molorehus n.

Crotalaria sagittalis.-Plooms June 14-Oetober 9: 8 visitors observed, June 16-July 9 ; Ma, Y.

Long-tongued Bees (6)-Bomb.: Bombus americanorum 6 ;

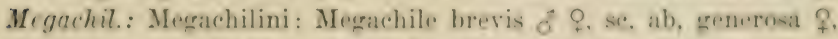
Sarogaster of, se; Coelioxyini : Coelioxys 8-dentata \&, sayi ?.

Lepidoptera (2, n)-Lycaen.: Everes; Papilion.: Pieris.

Desmodium bracteosum (3:83).-Blooms July 11-August 29; 3 visitors observed, August 13, 22; Ma, R.

Long-tomgned Bees (3, e)-Bemb.: Bembus amerieanorum Q: Meguchil.: Mrgachile brevis १; Eucer.: Melissodes bimaeulata 9.

Desmodium canadense $(3: 43)$.- Blooms June 1:-September 7; 6 visitors observed, July 11-August 25; Ma, R. 
Long-tongued Bees (6, c)-Bomb.: Bombias separatus $\Varangle$, Bombus americanorum $\not{\phi}, \mathrm{fq}$; Megachil $(q)$ : Megachile brevis fq, mendica, petulans; Eucer.: Melissodes bimaculata $ᄋ$.

Desmodium canescens.-Blooms July 11-September 7; 1 visitor observed, September 1; Ma, R.

Long-tongued Bees (1)-Megachil.: Megachile brevis ㅇ, c.

Desmodium dillenii (3:83).-Blooms July 30-September 7; 5 visitors observed, August 2-September 2; Ma, R.

Long-tongued Bees (5, c)-Bomb.: Bombus americanorum $\Varangle ;$ Megachil. (q) : Megachile brevis, mendica, petulans; Eucer.: Melissodes bimaculata .

Desmodium grandiflorum.-Blooms June 22-August $17 ; 1$ visitor observed, July 9 ; Mi, W.

Short-tongued Bees (1)-Halict.: Chloralictus versatus + , c.

Desmodium illinoense.-Blooms June 5-August 10; 9 visitors observed, June 30-July 9 ; Ma, R.

Long-tongued Bees (7)-Bomb. (ఫ, c) : Bombias auricomus, Bombus americanorum; Megachil.: Osmiini: Alcidamea simplex ○, e; Megachile brevis $\mathrm{fq}$, mendica, Sarogaster; Coelioxyini: Coelioxys 8-dentata, explor, $n$.

Short-tongued Bees (1)-Halict.: Chloralictus versatus $q$, esp, $n$.

Lepidoptera (1)-Lycaen.: Everes, explor, $n$.

Desmodium marilandicum (3:83).-Blooms August 22-September $3 ; 1$ visitor observed, August 22; Ma, R.

Short-torgued Bees (1)-Panurg.: Calliopsis andreniformis + , c.

Desmodium paniculatum (3: 83).-Blooms July 17-September 23 ; 8 visitors observed, August 7-September $10 ; \mathrm{Ma}, \mathrm{R}$.

Long-tongued Bees $(6, \mathrm{c})-$ Bomb.: Bombus americanorum $\Varangle$, impatiens $\Varangle ;$ Megachil. ( () : Megachile brevis, mendica, petulans; Eucer.: Melissodes bimaculata $q$.

Short-tongued Bees $(2,+$, c)-Panurg.: Calliopsis andreniformis ; Nomi.: Paranomia.

Desmodium sessilifolium (3: 83).-Blooms July 18-September 18; 2 visitors observed, August 14, 15; Ma, R.

Long-tongued Bees (1)-Megachil.: Megachile brevis $q$, c.

Short-tongued Bees (1)-Halict.: Chloralictus versatus $q$, csp, $\mathrm{n}$.

Gleditsia triacanthos.-Blooms May 4-June 5, 13 visitors observed, May 24-June 3; Mis, Y. 
Long-tongued Bees (2) - $.1 p .: A$ pis se, $\mathrm{fq} ;$ Bumb.: Bombias separatus $q$, se.

Short-tongued Bees $(6, q)$ - Haliet.: Agapostemon radiatus se, Chloralietus illinoensis, sparsus; Andren.: Andrena dunningii, Opandrena personata, Trachandrena claytoniae se.

Diptera (5)-Syrph.: Eristalis dimidiatus, Mesngramma geminata. Myiolepta varipes; Empid.: Rhamphomyia mutabilis; Anthomy.: Phorbia platura.

Gymnocladus dioica $(16: 165)$ - - Blooms May 7-June $1 ; 5$ visitors observed, May $10 ; \mathrm{Ma}, \mathrm{W}$.

Birds (1)-Trochil.: Trochilus.

Long-tongued Bees (3)-Bomb.: Bombus americanorum o, fq, impatiens $q$, se ; Eucer.: Tetralonia dilecta $\delta$.

Lepidoptera (1)-Papilion.: Euphoeades.

Lespedeza capitata (10: 231).-Blooms July 23-September 17 ; 4 visitors observed, August 14-26; $\mathrm{Ma}, \mathrm{R}$.

Long-tongued Bees (2)-Bomb.: Bombus amerieanorum $\Varangle ;$ Megachil.: Megachile brevis $q$, se.

Short-tongued Bees (1)-P'unurg.: Calliopsis andreniformis of. Lepidoptera (1)-Papilion.: Eurymus n.

Lespedeza procumbens (10:230).-Blooms July 17-September $21 ; 9$ visitors observed, August 7-25; $\mathrm{Ma}, \mathrm{R}$.

Long-tongrued Pees (4)-Megachil. ( $q$, se) : Megachile brevis, mendica; Pasit.: Holeopasites illinoensis $\delta^{+}$., $\mathrm{f} q$, type; Eucer.: Melissodes bimaculata ㅇ.

Short-tongued Bees (3)-IIalict. ( $($, sc) : Chloralictus versatus, Oxystoglossa confusa ; Panurg.: Calliopsis andreniformis of + .

Diptera (1)-Bombyli.: Systoechus.

Lepidoptera (1)-Lycaen.: Everes n.

Lespedeza virginica $(10: 230-1)$.-Blooms July 22-September 14; 21 visitors observed, August 7-September 7 : Ma. R.

Long-tongrued Bees (11)-Bomh.: Bumbus amrrieanorum of 8. se, fy; Megachil.: Megachilini : Megachile hrevis o \&. se, ab, jetulans ․ se. Xanthrarus 9; Coelioxyini: Coelioxys germana of; Epeol.: Triepenlus eoneavus $?$. simplex $\&$; Euctr.: Melissodes autumnalis $\hat{d}$, comptoides $q$, nivea $\delta$ \% , se; Anthophor.: Amegilla .

Short-tongued Bews (5)-Halict. $\left(\delta^{8}\right)$ : Odontalietus, Oxystoglossa enfusa, similis; Collet.: Colletes americanus $\delta$; Panurg.: Calliopsis andreniformis $\delta^{7}$ ?

Diptera (1)-Anthomy.: Ifylemyia laevis. 
Lepidoptera (4, n)-Lycaen.: Everes fq, Uranotes; Papilion.: Eurymus; Hesperi.: Limochores taumas.

Melilotus alba (6:473-4).-Blooms June 1-October 18; 153 visitors observed, June 20-July 4; Pol (Mis), W.

Long-tongued Bees (23)-Ap.: Apis ab; Bomb.: Bombias auricomus $q$, seutellaris $q$, separatus $q \not$, fq, Bombus americanorum $\uparrow \Varangle, f q$, Psithyrus variabilis $q$; Ceratin.: Ceratina $q$, sc ; Megachil.: Trypetini: Neotrypetes truncatus $q$; Osmiini: Alcidamea simplex + , se; Megachilini: Megachile brevis $\delta^{\lambda}+$, se, fq, petulans $\delta^{\lambda}$, 6-dentata $o^{\pi}$, Xanthosarus $\sigma^{\pi}$; Coelioxyini: Coelioxys 8-dentata $\sigma^{\pi}$, ab, rufitarsis $q$, sayi $q$; Xylocop.: Xylocopa $\sigma^{1}+$; Nomad.: Centrias americanus $q$, Holonomada affabilis $\delta q$, superba $q$, fq; Epeol.: Epeolus bifasciatus $\delta$ \% ab; Eucer.: Tetralonia dilecta $q$; Anthophor.: Anthemoessa ô.

Short-tongued Bees (21)-Halict.: Agapostemon radiatus $q$, viridulus $q$, Chloralictus albipennis $q$, se, pilosus $\delta^{\lambda}$, pruinosus $\delta^{\lambda}$, versatus $\delta^{\lambda} q, \mathrm{sc}, \mathrm{ab}$, Evylaeus areuatus $q$, sc, pectoralis $\delta^{\lambda}$, Halictus lerouxii $\delta^{\lambda}+$, se, parallelus $\delta^{\lambda}+$, Odontalictus $q$, se, Oxystoglossa

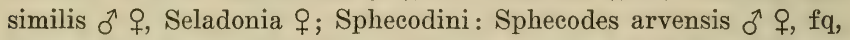
Sphecodium cressonii $q$; Collet.: Colletes eulophi ô $q$, fq, latitarsis

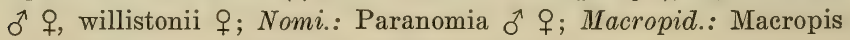
$\widehat{0}$ ㅇ, fq ; Panurg.: Calliopsis andreniformis $\widehat{\gamma}$, $q$, se.

Other Hymenoptera (52)-Sphec.: Ammobia ichneumonea, pennsylvanica, Isodontia apicalis, macrocephala, Priononyx atrata, thomae, Sceliphron, Sphex nigricans, pictipennis fq, procera, vulgaris; Bembic.: Bembix nubillipennis, Bicyrtes ventralis, Stizus fq ; Scoli.: Elis 5-cincta; Philanth.: Cerceris bicornuta, clypeata, compacta, finitima, prominens, Philanthus ventilabris; Vesp.: Polistes rubiginosus fq, variatus; Eumen.: Ancistrocerus tigris, Eumenes belfragei, fraternus, Leionotus anormis fq, arvensis, clypeatus, dorsalis $\mathrm{fq}$, foraminatus $\mathrm{fq}$, fulvipes, histrionalis, megaera, ziziae, Monobia; Crabron.: Solenius fq; Oxybel.: Notoglossa americana; Larr.: Tachytes elongatus ; Pompil.: Anoplius illinoensis, Batizonus, Ceropales bipunctata $\mathrm{f} q$, elegans, fulvipes, Episyron, Lophopompilus atrox, Poecilopompilus navus, Pompiloides americanus, marginatus, Priocnemoides fulvicornis, Psammochares relativus; Tiphi.: Tiphia vulgaris.

Diptera (36)—Taban.: Chrysops striatus; Stratiomy.: Stratiomyia meigenii ; Syrph.: Allograpta, Eristalis aeneus fq, latifrons, Helophilus latifrons, Platychirus quadratus, Sphaerophoria, Syritta, Syrphus americanus; Empid.: Parempis ; Conop.: Conops brachyrrhyncus fq, xanthopareus, Oncomyia, Physocephala texana, Stylo- 
gaster bianmulata, Zodion nanellum; Tachin.: Arehytas analis, aterrima Belvosia bifasciata, Cistogaster oceidua, Cyphocera ab, Euphoroeera elaripennis, Linmaenyia ab, Oeyptera lata, fq. l'arlyophthalmus floridensis, signatus, Sinotainia rubriventris, Spallanzania, Trichophora, Winthemia; Sareopheg,: Helicohia helieis. Sareophaga eimbicis; Muse.: Morellia, Phormia regina, Porulepyrellia.

Lepidoptera (14, n)-.ymphal.: Anosia, Vanessa atalanta, huntera; Lycatn.: Chrysophanus, Cyaniris, Strymon, Iranotes; Papilion.: Callidryas, Iphiclides, Papilio, I'ontiat Heperi.: Epargyreus, Limochores taumas; Sesi.: Sesia 6-fasciata.

Coleoptera (5)-Cerambye. Typoecrus sinnatus: Cureulion.: Centrimus pieumnus, scutellum-allum fq; Mordell.: Mordella marginata; Scarabae.: 'Trichius piger.

Hemiptera (2)-Lygar.: Iygaeus; Pentulom.: Podisus.

Petalostemum candidum.-Blooms June 20-August 30 ; 30 visitors observed, July 8-19; Mis, WV.

Long-tongued bees (4)- 1 p.: Apis; Bomb.: Bombias aurieomus $\not$, seutellaris $\not$, separatus $q \not \gamma, \mathrm{fq}$.

Short-tongued Bees (11)-IIulict.: Agapostemon radiatus f,

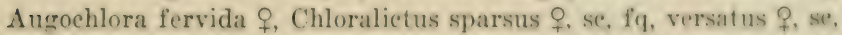

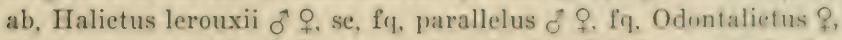
Oxystoglossa eonfusa $q$, se, similis $q$, $f(q$, Seladonia $f$ ?. fq: Prosopid.: Prosopis ziziae $q$.

Other Hymenoptera (7)-Sphre.: Ammobia iehnemunea, Priononyx atrata; Bembic.: Bicyrtes 4-fasciata; Scoli.: Elis interrupta, 5-cincta ab; Philanth.: Cerceris bicormuta, Philanthus vemtilabris.

Diptera (7)-Syrph.: Allograpta, Tropidia quadrata; Mila.: Midas elavatus; Conop.: Conops xanthopareus fq. Zodion namellum : Tachin.: Archytas analis; Musc.: Compsomyia.

Lepidoptera (1)-Lycaen.: Everes.

Petalostemum purpureum (3:81-2),-Blooms Jun 27-August 10 ; 94 visitors observed, July $1-$ August 2 ; Mas, R.

Lone-tongned Beres (37) - Ap.: Apis se, ab; Bumb.: Bombias

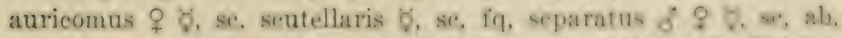
Bombus amerieanorum of $q \nabla . \mathrm{se}, \mathrm{ab}$, impatiens ? of. se. in, l'aithy. rus variabilis f; Ceratin: Ceratinn o, se; Megachil: Trypetini: Neotrypetes truncatus ?. se; Osmiini: Mcidamea simples \&. se, Inoplitis 8. se. fq; Megachilini: Megachile hrevis of Q, se, ah. 6ralendata 8 . Sayapis sayi \&. Xanthosarus of 9. se. Py: Corlioxyini: 
Coelioxys alternata $q$, germana $q, 8$-dentata $\delta^{\lambda} q$, fq, rufitarsis $\sigma^{\lambda} q$, sayi $q$; Xylocop.: Xylocopa $q$; Epeol.: Epeolus bifaseiatus + , Triepeolus concavus $\delta^{\lambda} q, f q$, concolor $\delta^{\lambda} q$, ab, lunatus $\delta^{\lambda}$, remigatus $q$; Eucer.: Epimelissodes obliqua $\delta^{\lambda}+$, se, fq, Melissodes agilis $\delta$, bimaculata $\delta^{\lambda}+\mathrm{sc}, \mathrm{ab}$, comptoides $\widehat{\delta} q, \mathrm{fq}$, petalostemonis $q, \mathrm{se}, \mathrm{fq}$, type, trinodis $\hat{\sigma}$, variabilis $\hat{\delta} q, \mathrm{se}, \mathrm{fq}$, vernoniana $\hat{\sigma} q$, Tetralonia dilecta + , se, Xenoglossodes + ; Anthophor.: Amegilla $\sigma^{\top}$.

Short-tongued Bees (18)-Halict.: Agapostemon radiatus $q$, splendens $q$, texanus $\sigma^{\top} q$, viridulus $q$, se, ab, Augochlora fervida $q$, se, fq, Chloralictus albipennis + , e, fq, pilosus $q$, se, pruinosus $q$, versatus $q$, se, fq, Halictus lerouxii $\delta^{\lambda} q, \mathrm{se}, \mathrm{fq}$, parallelus $\delta^{\lambda} q, \mathrm{se}, \mathrm{ab}$, Odontalictus $q$, se, Oxystoglossa confusa $q$, se, similis $q$, se, ab, Seladonia $\uparrow$, sc, ab; Collet.: Colletes albescens + , se, oligolege, robertsonii $\delta^{\top}+$, sc, oligolege, type; Nomi.: Paranomia ot.

Other Hymenoptera (15)—Sphec.: Ammobia ichneumonea, Priononyx atrata, thomae, Sphex gracilis, nigricans $f q$, pictipennis, procera, vulgaris; Bembic.: Bembix nubillipennis, spinolae; Scoli.: Campsomeris, Elis 5-cincta fq; Philanth.: Cerceris bicornuta, Philanthus ventilabris; Eumen.: Eumenes fraternus.

Diptera (12)—Syrph.: Allograpta, Eristalis latifrons, Helophilus latifrons, Mesogramma marginata, Sphaerophoria, Tropidia quadrata; Bombyli.: Exoprosopa 'fasciata, Systoechus; Conop.: Oncomyia, Physocephala texana, Zodion obliquefasciatum; Sarcophag.: Sareophaga cimbicis.

Lepidoptera (6)-Lycaen.: Everes; Papilion.: Eurymus, Zerene; Hesperi.: Limochores taumas, Pholisora catullus, Polites.

Coleoptera (4)-Cerambyc.: Typocerus sinuatus; Chrysomel.: Diabrotica 12-punctata; Melo.: Epicauta pennsylvanica, trichrus.

Hemiptera (2)-Caps.: Calocoris; Pentatom.: Euschistus ictericus.

Psoralea onobrychis (3:79-80).-Blooms May 27-July 31; 55 visitors observed, June 15-July 16 ; Mas, $R$.

Long-tongued Bees (27)-Ap.: Apis fq; Bomb.: Bombias auricomus $\Varangle$, scutellaris $q$, separatus $q \varnothing$, se, fq, Bombus americanorum

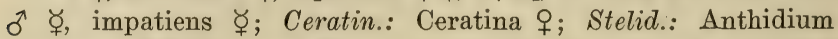
psoraleae $\sigma^{\lambda} q$, fq, type; Megachil.: Osmiini : Alcidamea simplex $q$, sc, Diceratosmia $q$, Osmia atriventris $q$; Megachilini: Chelostom-

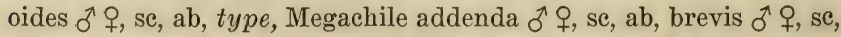

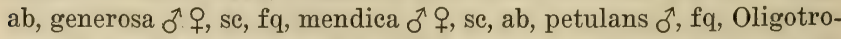
pus $\delta$, ab, Xanthosarus $\delta$; Coelioxyini: Coelioxys germana $\delta^{\lambda}$, modesta $\delta^{\lambda}+$, fq, 8-dentata $\sigma^{\lambda}+$, fq, sayi $\sigma^{\lambda}+$, fq ; Epeol.: Epeolus interruptus $q$, type; Eucer.: Florilegus 0 , Melissodes bimaculata $\delta$, Tetralonia dilecta . 
Short-tongued Bees (8)-IIalict.: Agapostemon radiatus of $f$. se, Curtisapis coriacea of Evylaeus areuatus \&. Halietus lerouxii of. Seladonia of ?, in eop; Collet.: Colletes brevicornis of fq, eulophi $\sigma^{\circ}+\mathrm{se}, \mathrm{ab}$, in eop ; P'unurg.: Calliopsis andrenifurmis $6 \%$ se, ab, in eop.

Other Hymenoptera ( 7$)-S p h e c .:$ Priononyx atrata, thomae, Sphex gracilis; Scoli.: Elis 5-eincta; Eumen.: Leinotus arvensis, dorsalis, Pterochilus.

Diptera (7)—Syrph.: Tropidia mamillata: Bombyli.: Anthrax parvicornis, sinuosa fsp, Bombylius atriceps, Exoprosopa fasciata; Conop.: Physoeephala tibialis, Stylogaster biannulatia.

Lepidoptera (6. n)-Pupilion.: Laertias, Pontia: IIsperi.: Epargyreus, Thanaos juvenalis; $S$ phing.: Hemaris thyshe; Not u.: Schinia.

Robinia pseudacacia (int).-Blooms April 21-May 2s; 11 visitors observed, May 9-25; Ma, R.

Birds (1) - Trochil.: 'Trochilus ab.

Long-tongued Bees $(\bar{T})-A p$.: Apis ; Bomb. ( $q)$ : Bombias auricomus fq, seutellaris, separatus, Bombus americanorum fq, impatiens ab; Eucer.: Tetralonia dilecta .

Lepidoptera (3, n)-Nymphal.: Anosia ; Pupilion.: Iphiclides: Sphing.: Deilephila.

Strophostyles helvola $(3: 199)$.-Blooms July 7 -Oetober 19 ; 8 visitors observed, July $31-\Lambda$ ugust $26 ; \mathrm{M}$ a, $\mathrm{R}$.

Long-tongued Bees ( 8$)-B o m b$.: Bombus americanorum $₹$ :

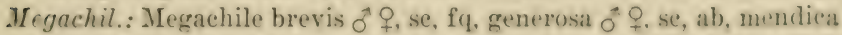
$\delta^{3}+$. petulans $q, \mathrm{se}, \mathrm{fq}$, strophostylis $q, \mathrm{se}, \mathrm{fq}$, oligolege, type, Oligotropus $\delta$, Xanthosarus ․

Estra-floral nectaries. - The following 4.5 species were taken on the extra-floral nectaries, July 31-September 8:

Ints (3. 8)-Formic.: Formica fusea ab, schaufussi, Lasius.

Short-tongued Bees (2, \&)-Halict.: Oxystoglosiat eonfusa; Sphecodini: Sphecodes arvensis.

Other Hymenoptera (23) - Sphec: Chalybion, Seeliphron; Philanth.: Cereeris elypeata, finitima, kennicottii fq. Philanthus punctatus: Vesp.: Polistes variatus. Vespula germanica; Eumen.: Leionotus vagus; Orybel.: Oxybelus 4-notatus: Tryporyl.: Trypoxylon frigidum; Larr.: Astatus unieolor, Tachysphex mundus; Nysson.: Zanyson aurinotus; Pompil.: Agoniella longula, Jophopompilus atrox; Mutill. (d): Mutilla scrupea, Sphaerophthalma; Chrysid.: Hedyehrum parvum, Tetrarhrysis renuata; Brocon.: Agathis vulgaris; Lchne umen.: Diplazon, Limmeria fura. 
Diptera (15)-Syrph.: Mesogramma marginata; Tachin.; Euphorocera claripennis, Paradidyma singularis type; Sarcophag.: Helicobia helicis, 4-setosa, Metoposareophaga, Sarcophaga assidua, hunteri, marginata; Musc.: Pseudopyrellia; Anthomy.: Coenosia antica; Agromyz.: Lobioptera; Drosophil.: Drosophila; Ortal.: Camptoneura, Rivellia 4-fasciata; Seps.: Sepsis.

Coleoptera (1)-Coccinell.: Coccinella 9-notata.

Hemiptera (1)-Caps.: Lygus.

Strophostyles pauciflora.-Blooms July 11-October 15; 3 visitors observed, August 14, September 7, October 3; Ma, R.

Long-tongued Bees (2)-Bomb.: Bombus americanorum לॄ; Megachil.: Megachile brevis .

Short-tongued Bees (1)-Panurg.: Calliopsis andreniformis ㅇ.

Extra-floral nectaries.-Three visitors observed, July 29, September 7 .

Ants (1)-Formic.: Formica schaufussi $\not,, 7$.

Short-tongued Bees (2)-Halict.: Chloralictus sparsus $\sigma^{\lambda}, 2$, versatus $\delta$ $q, 6$.

Stylosanthes biflora $(16: 163-5) .-$ Blooms June 8-September 9 ; 3 visitors observed, July 2-11; Ma, Y.

Long-tongued Bees (1)-Bomb.: Bombus americanorum ఫ్.

Short-tongued Bees (1)-Panurg.: Calliopsis andreniformis o, fq.

Lepidoptera (1)-Papilion.: Eurema n.

Tephrosia virginiana $(3: 82)$.-Blooms June 10-July $2 ; 5$ visitors observed, June 15-29; Ma, W.

Long-tongued Bees (5)-Megachil.: Chelostomoides $q$, sc, Megachile addenda $\sigma^{\top}+, \mathrm{se}, \mathrm{ab}$, brevis $q, \mathrm{se}$, mendica $q$, petulans $\sigma^{\top}+$.

Trifolium hybridum (int).-Blooms May 14-June 25; 16 visitors observed, June 6-18; Mas, W.

Long-tongued Bees (6)-Ap.: Apis se, fq; Megachil. (,$+ \mathrm{sc})$ : Alcidamea simplex fq, Diceratosmia, Osmia cordata; Nomad.: Holonomada affabilis + ; Epeol.: Epeolus interruptus + .

Short-tongued Bees (5)-Halict. ( $(+)$ : Chloralictus versatus se, Odontalictus, Oxystoglossa similis se, Seladonia se; Panurg.: Calliopsis andreniformis $\delta$ ㅇ․

Other Hymenoptera (1)-Sphec.: Priononyx atrata.

Diptera (1)-Conop.: Zodion fulvifrons n.

Lepidoptera $(3, \mathrm{n})$-Nymphal.: Charidryas; Lycaen.: Chrysophanus; Hesperi.: Ancyloxypha. 
Trifolium pratense (int, 5:177-8).-Blowns April 26-Nivember $1 ; 42$ visitors observed, May 21-September 11; Mas, R.

Birds (1)-Trochil.: Trochilus.

Long-tongued Bees (15)-Bomb.: liombias aurieomus \& 8 .

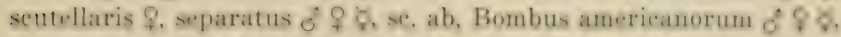
se, ab, eousimilis 8, se, inpatiens \&, rillingsii \&. Psithyrus laborion sus \&, variahilis \&. fy; stelid.: Anthidium psoraleae of \&, ar, fị; Megochil. (8): Osmini: Aleidamea simples, Osmia cordata se; Megaehilini : Megaehile brevis se; Eucer.: Melissodes bimaeulata \&, Tetralonia dileeta of se, fy: Anthophor.: Amegilla ?. Anthemumana 8 \% Anthophora $q$, se.

Short-tongued Bers (2, ㅇ, n)-Maliet.: Inalietus lerouxii f; Panurg.: Calliopsis andreniformis e.

Diptera (1)-Bombyli.: Exoprosopa fasciata.

Lepidoptera (20, n)-Vymphal.: Anosia, Arrynnis, Vane-sa atalanta, eardui, huntera fq : Lycuen.: Everes; Pupilion.: Callidryas, Eurymus, Iferaclides, Iphielides, Laertias, Papilio, Picris, Poutia, Xanthidia; IIesperi.: Epargyreus ab, Limoehores taumas, Polites, Thorybes pylades; Sphing.: Hemaris axillaris.

Trifolium reflexum.-Blooms May 22-June 22; 2 visiturs ob. served, June 13; Mas, W.

Long-tongued Bees (1)-Megachil.: Osmia atriventris \&.

Lepidoptera (1)-Lycaen.: Cyaniris n.

Trifolium repens (int).-Bloums April 23-Oetober 11 ; 80 viitors observed, May 12-July 14; Mas, W.

Long-tongued Bers (33) - Ap.: Apis ab; Bomb.: Bombias s.paratus 5, se, Bombus americanorum $\$ 8$, se, impatims $\$ 7$, ridingsi

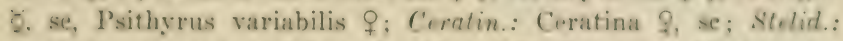
Anthidium psoralene \& \&. sc, fq : Miguchil.: Trypetini : Neotrypetes barbatus d: Osmini : Aledamea simplex of $\&$, se, ab, Direratosmia

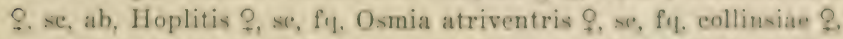
se, cordata \&, distineta \&, se, pumila ?, se, ab; Megachilini : Anthrs mois of. Megachile brevis of q. se. fq. genernsa d, mendiea d;

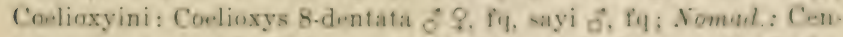
trius americanus ?. Holonomada affabilis of \&. fq. superba ?. fo: Epeol.: Fpeolus bifaseiatus d. interruptus $d$ ? Triepeolns rmiga. tus d; Fuere.: Tetralonia dilecta d, rosae ?; Anthequer: Anthe. moessa of, Anthophora $q$, se.

Short-tongued Bres (15)-Malict: A Aapostemon raAiatus \&, ac, fy, viridulus of, se, Auguehlora fervida ?, virilula of, Chloralietus pilosus 9. se. versatus 9. se. Curtiapis coriacea 9. *. fq. Halietus 
lerouxii ơ + , se, fq, parallelus $q$, se, Odontalictus $q$, se, Oxystoglossa confusa $\sigma^{\lambda} q$, fq, similis $q$, Seladonia $\delta^{\lambda} q$, se, fq ; Andren.: Opandrena cressonii $q$, se ; Panurg.: Calliopsis andreniformis $\sigma^{\lambda}+$, se, fq.

Other Hymenoptera (6)-Sphec.: Priononyx atrata, Sphex nigricans, pictipennis, procera; Bembic.: Microbembex; Scoli.: Campsomeris.

Diptera (9)-Empid.: Parempis; Bombyli.: Bombylius atriceps, Systoechus, Thlipsogaster; Conop.: Physocephala texana, tibialis, Stylogaster biannulata, Zodion fulvifrons; Tachin.: Archytas analis.

Lepidoptera (17, n)-Nymphal.: Charidryas, Euptoieta, Vanessa huntera; Lycaen.: Cyaniris, Everes; Papilion.: Eurymus, Iphiclides, Pieris, Pontia; Hesperi.: Ancyloxypha, Epargyreus, Limochores taumas fq, Pholisora catullus, Polites, Thanaos juvenalis, Thorybes pylades; Noctu.: Plusia simplex.

\section{LiLIACEae}

Asparagus officinalis (int) -7 visitors observed, May 11August $5 ; \mathrm{Ma}, \mathrm{Y}$.

Long-tongued Bees (2)-Ap.: Apis; Megachil.: Anthemois $\delta^{7}$,, se, ab.

Short-tongued Bees $(3$, ㅇ)-Halict.: Chloralictus versatus se, zephyrus sc, Oxystoglossa similis c.

Camassia esculenta $(9: 266-7)$.-Blooms April 22-May 28; 48 visitors observed, April 29-May 24; Mi (Ma), R.

Long-tongued Bees (15)-Ap.: Apis ab; Bomb. (q) : Bombias auricomus, separatus, Bombus americanorum, impatiens; Ceratin.: Ceratina $\delta^{\lambda}$, Zaodontomerus $\delta^{\lambda}$; Megachil.: Osmiini: Ceratosmia $q$, Osmia pumila $\delta^{\lambda}+$; Megachilini : Megachile mendica $\delta$ \% ; Nomad.: Centrias americanus $\delta^{\lambda}$, Holonomada affabilis $q$, superba $\delta$, $f q$; Eucer.: Tetralonia belfragei, 0 , dilecta $\delta$, fq.

Short-tongued Bees $(11, q)$ - Halict.: Agapostemon viridulus, Chloralictus pilosus se, ab, pruinosus se, fq, versatus se, fq, Curtisapis forbesii, Evylaeus pectoralis fq, Halictus lerouxii se, Odontalictus sc, Oxystoglossa confusa, similis, Seladonia sc, ab.

Other Hymenoptera (4)-Vesp.: Polistes variatus; Eumen.: Ancistrocerus tigris, Leionotus clypeatus; Pompil.: Anoplius illinoensis.

Diptera (12)—Syrph.: Eristalis dimidiatus, Orthoneura nitida, pictipennis, Paragus bicolor, Syritta; Tachin.: Linnaemyia; Sarcophag.: Cynomyia, Helicobia helicis; Musc.: Lucilia sericata, Phormia regina; Anthomy.: Phorbia acra, platura. 
Lepidoptera (5) - Vymphal.: Charidryas, Vanessa atalanta, huntera; Pupilion.: Eurymus fq; Hesperi.: Pholisora eatullus.

Coleoptera (1)-Coccinell.: Hippodamea 1.j-manulata.

Erythronium albidum (5:69-71).-Blumus Mareh 17-11ay "2; 28 visitors observed, March $2 \pm$ April $22 ; \mathrm{Mi}, \mathrm{W}$.

Long-tongued Bees (11)-Ap.: Apis se, ab; Bomb.: liombus impatieus 9 ; Ceratin.: ('eratina of Megachil.: Cientrosmia d. Cera-

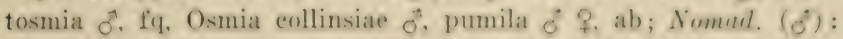
Xanthidium dentariae, luteoloides, luteolum: Euer.: Tetralonia belfragei $\delta$, fq.

short-tongued Bees (9) - Halict. (f) : Chloralietus pilosus, versatus se, type, IIalietus lerouxii, Seladoniat Collet.: Colletes inaequalis $\hat{\delta}$, ab; Andren.: Andrena carlini $\delta$ o, se, ab, erythronii

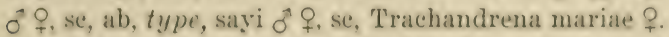

Diptera (4)-Xyrph.: Brachypalpus frontosus f, n. Eristalis dimidiatus; Bombyli.: Bombylius major; Musc.: Pseudopyrellia $\mathrm{fq}, \mathrm{n}$.

Lepidoptera (3)-Papilion.: Eurymus, Pieris; Hesperi.: Thanaos juvenalis.

Hemiptera (1)-Caps.: Lygus n.

Lilium superbum.-Blooms June 15-July 17; :3 visitors observed, July 2 ; Ma, R.

Lepidoptera.-Nymphal.: Anosia, Argynnis; Papilion.: Euphoeades.

Melanthium virginicum $(9: 27: 3-4)$.-Blooms June 12-July 11 ; 30 visitors observed, June 27-July 5 ; Mi, W.

Short-tongued Bees (1) - Halict.: Chloralictus rersatus f. n.

Other Hymenoptera (3) -Sphec.: Ammobia ichnemmonea; Chalcill.: Perilampus triangularis n; Tenthrolin.: Hylutoma humeralis.

Diptera (19) —Stratiomy.: Stratiomyia meigenii; Syrph. (n): Mesogramma marginata, Sphaerophoria, Syritta; Empil.: Parempis n; Tachin.: Archytas aterrima, Cistogaster oceidua, pallasii, Enphorocera elaripennis, Limnatemyia, Paradidyma n. Trichopoda [u-nnipes; Sarcophag.: Sareophaga cimbieis: Musn.: Lueilia carsar, Musea, Pseudopyrellia; Anthomy.: Anthomyiella n, Calythea n; Oscin.: Chlorops mellea $\mathrm{n}$.

Lepidoptera (1)-Lycaen.: Cyaniris $\mathrm{n}$.

Coleoptera (6)-Chrysomel.: Diabrotica atripennis u; Curculion.: Centrinites fq. n; Lampyr.: Photimus: Mordell.: Mordella marginata n, melaena fq. $\mathrm{n}$; Scaruhar.: Trichius piger, fq. 
Nothoscordum bivalve $(9: 266)$.-Blooms April 9-May 18; 19 visitors observed, April 20-May 9; Mi, W.

Long-tongued Bees (9)-Megachil.: Osmia atriventris $\delta^{\star}$; Nomad.: Centrias americanus $\delta$, Gnathias cuneatus $q$, ovatus $\delta q$, fq, Holonomada superba $\widehat{\delta}, \mathrm{fq}$, Nomada cressonii $\delta \hat{~}+$, ab, type, parva $\sigma^{\lambda}$, fq, sayi $q, f q$, Xanthidium luteoloides $\delta^{\top}$.

Short-tongued Bees $(3, q)$ - Halict.: Chloralictus versatus se, fq, Oxystoglossa similis ab; Andren.: Andrena nothoseordi, se, type, oligolege.

Diptera (3) - Syrph.: Mesogramma marginata, Sphaerophoria $\mathrm{fq}$; Bombyli: : Bombylius pulchellus.

Lepidoptera (4)-Lycaen.: Everes; Papilion.: Eurymus, Pieris ; Noctu.: Plusia simplex.

Polygonatum commutatum (9:268-9).-Blooms May 11-June 23; 7 species and 23 individuals observed, May 23-June 9 ; Ma, W.

Birds (1:4)-Trochil:: Trochilus 4.

Long-tongued Bees $(5: 18)-B o m b .:$ Bombus americanorum + , 5, consimilis + , se, 4 ; Ceratin.: Ceratina $q$, se, 2 ; Anthophor.: Anthemoessa $\delta$ \% , sc, 4, Anthophora $q$, sc, 3.

Short-tongued Bees $(1: 1)$-Halict.: Oxystoglossa confusa o, $\mathrm{e}, \mathrm{n}$.

Smilacina racemosa $(9: 270)$._-Blooms May $7-30 ; 21$ species and 44 individuals observed, May 8-23; Mi, W.

Long-tongued Bees $(1: 1)-A p .:$ Apis c, 1.

Short-tongued Bees ( $7: 13)$-Halic. (q): Chloralictus obscurus c, 2, sparsus c, 2, versatus c, 1, zephyrus 1, Evylaeus pectoralis c, 4, 4-maculatus c, 2, Oxystoglossa pura sc, 1.

Diptera (8: 19)-Culic.: Culex nigripes 1; Syrph.: Chilosia cyanescens $\mathrm{f}$, 1, Criorhina decora 1, Volucella vesiculosa 5 ; Empid.: Empis humilis 3, levicula 1; Tachin.: Siphona 4; Musc.: Lucilia caesar 3.

Coleoptera (5: 11)-Anthic.: Corphyra terminalis 1; Bruch.: Bruchus mimus 5; Mordell.: Mordellistena biplagiata 3; Scarabae.: Trichius affinis 1 , Valgus 1 .

Smilacina stellata $(9: 269-70)$.-Blooms April 25-May 12; 16 species and 28 individuals observed, April $30 ; \mathrm{Mi}, \mathrm{W}$.

Long-tongued Bees (2: 2, $\left.\sigma^{1}\right)$-Ceratin.: Zaodontomerus 1; Nomad: Nomada cressonii 1.

Short-tongued Bees $(12: 20$, 우).-Halic.: Augochlora viridula sc, 1, Chloralictus obscurus sc, 1 , smilacinae se, 1, type, sparsus sc, 1, Evylaeus 4-maculatus sc, 5, Halictus lerouxii sc, 1, Oxystoglossa 
eonfusa se, 4, pura se, 1, similis se, 1; Sphroodini: Spherodium smilaeinae 1, tupe: Indren.: Andrena earlini 1, Upandrena rressonii se, 2.

Diptera (2: 6)--Empid.: Empis humilis 5; Bombyli.: Hombylius major 1 .

Smilax herbacea.-1ilooms May 24-July 6; 9 spueies and 19 individuals observed, May 30, June 4, 6, 8; Mi, Y.

On staminate flowers:-

Long-tongued Bees $(1: 1)-\Lambda p .: \Lambda$ pis c, 1 .

Short-tongued Bees (3: 11, q, se)-Maliet.: Chlomalietus versatus 3 , Oxystoglossa confusa 1 , similis 7 .

Diptera (2: 4)-Surcophag.: Sareophaga eimbicis 3; Muse.: Lucilia caesar 1.

Lepidoptera $(1: 1)-$ Nymphal.: Neonympha 1.

Coleoptera (2: 2. f. n)-Cerambuc.: Euderees 1; Scarabue.: Euphoria fulgida 1.

Smilax hispida.-Blonms May 3-June :3; 16 visitors observal, May 17-June 3 ; Mi, Y.

Long-tongued Bees (5) - Ap).: Ap is se; Bomb.: Bombus impatiens Q; Coratin.: Ceratina q. se; Megachil.: Osmia pumila f. se ; Nomad.: Nomada parva $\delta$.

Short-tongued Bees (6. P)-IIalict.: Aerpostemon radiafus $e$. Chloralictus tegularis se, zephyrus e, Evylaeus pectoralis e, Osysto. glossa confusa se; Andren.: Opandrena cressonii se.

Diptera (5)-Syrph. (f): Allograpta. Mesogramma geminata, Syrplus americanus; Empid.: Empis loripedis; Conop.: Conops brachyrrhynchus.

Stenanthium angustifolium.-Blooms Jume 24.July 24; 4 vis. itors observed, July 14, 16; Mi, W.

Short-tongued Bees (2)--Malict.: Chloralietus sparsus $q$ : Prosopid.: Prosopis ziziae $\delta$.

Diptera (2)—Syrph.: Syritta ; Tuchin.: Oryptera lata.

Trillium sessile $(9: 271-3)$.- Blooms April $24-$ May $15 ; 1$ vis. itor observed, April 29, Mi, R.

Coleoptera (1)-Curculion.: Centrinites f, 10.

Uvularia grandiflora $(9: 270-1)$. - Blooms April 12 May $11: 8$ species and 19 individuals ohsorved. April 17-2y; Ma. Y.

Long-tomgued Bews (5:9, 2)-Bomb.: Bombias soparatus 1. Bumbus americanorum 2., impatiens 1, ridingsii t, Megachif : Osmia atriventris 1 . 
Short-tongued Bees (3: 10)-Andren.: Andrena carlini $\sigma^{1}$ ㅇ, sc, 7 , mandibularis + , se, 2 , pruni $\delta, 1$.

\section{LOBELIACEAE}

Lobelia cardinalis (4:68-9).-Blooms August 3-September 15; 6 visitors observed, August 18-September 2; Ma (0), R.

Birds (1) - Trochil.: Trochilus ab.

Long-tongued Bees (1)-Bomb.: Bombus americanorum $\Varangle, f q$, $\mathrm{s}$, through slits, $\mathrm{n}$.

Short-tongued Bees ( + , c, n)-Halict.: Chloralictus versatus; Oxystoglossa confusa.

Lepidoptera (2)-Papilion.: Euphoeades, Laertias.

Lobelia cardinalis $\times$ siphilitica $(4: 69)$. - Two visitors observed, August 21.

Birds (1)-Trochil.: Trochilus.

Long-tongued Bees (1)-Bomb.: Bombus americanorum $\Varangle$.

Lobelia inflata.-Blooms July 7-November $19 ; 2$ visitors observed, August 15; Mi, W.

Short-tongued Bees (2, ) - Halict.: Chloralictus sparsus, Oxystoglossa confusa.

Lobelia leptostachys (4:67-8).-Blooms June 30-August 17; 22 visitors observed, July 3-31; Mas, W.

Long-tongued Bees (14)-Ap.: Apis; Bomb. (૪) : Bombias

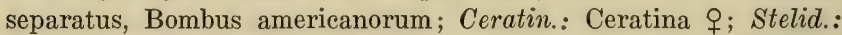
Anthidium psoraleae $\hat{\delta}$ 우 Megachil.: Osmiini: Hoplitis $q$; Megachilini : Chelostomoides $\delta^{\lambda}$, Megachile brevis $\delta^{\lambda}+$, sc, petulans $\sigma^{\lambda}, \mathrm{fq}$, Oligotropus $\delta$; Coelioxyini: Coelioxys 8-dentata $\delta$, rufitarsis $q$; Eucer.: Epimelissodes obliqua 0 , Melissodes bimaculata ${ }^{7}$.

Short-tongued Bees (4)-Halict.: Agapostemon viridulus $q$, Chloralictus pilosus $q, \mathrm{c}, \mathrm{n}$, Oxystoglossa confusa ${ }^{\lambda}+$, Seladonia $\delta$.

Diptera (1)-Bombyli.: Systoechus.

Lepidoptera (3)-Lycaen.: Everes; Hesperi.: Limochores taumas, Thanaos juvenalis.

Lobelia siphilitica $(4: 68)$.-Blooms August 12-October $16 ; 10$ visitors observed, August 21-September 17; Ma, R.

Long-tongued Bees (5)-Bomb.: Bombias separatus + , Bombus americanorum $q \not{q}, \mathrm{se}, \mathrm{ab}$, consimilis $\Varangle, \mathrm{se}$, impatiens $\Varangle, \mathrm{se}$; Anthophor.: Amegilla ㅇ.

Short-tongued Bees $(3,+9, \mathrm{n})$-Halict.: Chloralictus versatus c, Curtisapis coriacea, at slits, Oxystoglossa confusa c.

Lepidoptera (2)-Nymphal.: Anosia; Papilion.: Laertias. 
Lobelia spicata (4:67).-Blooms May 20-Jnne 30; 11 visitors observed, May 31-June 12; Mas, W.

Long-tongued Bees (4, q)-Ceratin.: Ceratina; Megachil.: Aleidamea simplex, Megachile brevis; Eucir.: Tetralunia dilerta.

Lepidoptera (7)-Lycarn.: Chrysuphanus; Papilion.: P'ieris, Pontia; Hesperi.: Aneyloxypha, Limochores taumas, Pholisora eatullus, Polites.

\section{Lyturaceae}

Ammannia coccinea.-Blooms June 25-October 6; 12 species and 21 individuals taken, July 31-August 30; Mi, R.

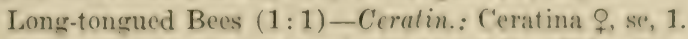

Short-tongued Bees $(5: 12)$ Halict. (q): Chloralietus pilosus se, 2, sparsus 1, versatus se, 7 , Oxystoglossa similis se, 1 ; Panurg.: Calliopsis andreniformis $q, 1$.

Diptera (3)-Syrph.: Mesogramma marginata 1, Syritta 1; Tachin.: Spallanzania 1.

Lepidoptera (3)-Lycacn.: Everes 1; Papilion.: Eurema 1, Pontia 1.

Cuphea petiolata.-Blooms July 15-Oetrober 26; 3 visitors observed, July 29-September 4; Ma, R.

Long-tongued Bees (2)-Bomb.: Bombus amerieanorum oै ab; Eucer.: Melissodes bimaculata ơ $q$, fq.

Short-tongued Bees (1)-IIulict.: Oxystoglossa similis $q$, se, $n$.

Lythrum alatum $(5: 179)$.-Blooms May 27-September $5 ; 55$ visitors observed, June 18-August 28; Mas, R.

Long-tongued Bees (24)-Ap.: $A_{\text {p }}$ is $\mathrm{fq}_{\mathrm{q}} ;$ Bomb.: Bombias se $\mathrm{p}$ aratus $q \varnothing$. Bombus impatiens $\Varangle$, se, fq; Ceratin.: Ceratina $q$; Megachil.: Osmini : Alcidamea simplex $\%$; Merachilini : Merachile

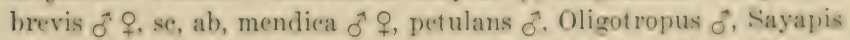
sayi of : Cuelioxyini : Coelioxys modesta $\%$. S-dentata of $\%$. iq ; Epeol.: Epeolus bifasciatus of $q$. Triepeolus concavus de coneolor $d ?$ : Eucer.: Epimelissodes atripes ${ }^{\prime}$, ohliqua d. Florilegus of $f$, Melissorles bimarulata $\hat{d}$, coloradensis of, comptoides of \&. se. fq, type. petalostemonis P. se, variabilis d. Tetralonia dilecta of ?.

Short-tongued Bees ( 7 -Malict.: Agajostemon radiatus of?.

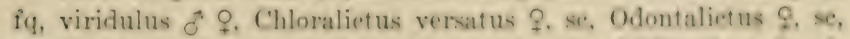
Oxystoglossa confusa of Seladonia $\delta$; Panurg.: Calliopsis andrniformis $q$, se.

Other Hymenoptera (3)-sphec: Priononyx atrata, Sphex nigricans $\mathrm{fq}$, procera. 
Diptera (9)—Syrph.: Eristalis latifrons, Helophilus latifrons f, n, Tropidia mamillata f, n; Bombyli.: Exoprosopa emarginata, fasciata fq, fascipennis, Geron rufipes, Systoechus; Tachin.: Archytas analis.

Lepidoptera (12)-Nymphal.: Charidryas, Vanessa cardui; Lycaen.: Chrysophanus; Papilion.: Eurema, Eurymus fq, Pieris, Pontia fq; Hesperi.: Aneyloxypha, Hesperia, Limochores taumas, Pholisora catullus fq, Polites fq.

\section{Malvaceae}

Abutilon theophrasti (int, 6:269-70).-Blooms June 26-October 14; 23 visitors observed, August 15-September 19; Ma, Y.

Birds (1) - Trochil.: Trochilus.

Long-tongued Bees (15)-Ap.: Apis; Bomb.: Bombias separatus

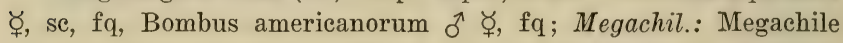
brevis + , Xanthosarus $\rho$, sc ; Eucer.: Epimelissodes obliqua $\sigma^{\lambda}$, Melissodes agilis $\delta$, bimaculata $q$, sc, fq, boltoniae $q$, comptoides $q, \mathrm{sc}$, nivea $q$, sc, petalostemonis $q$, simillima $\delta$, variabilis $q$, se; Anthophor.: Amegilla ㅇ․

Short-tongued Bees $(3, q)$-Hatict.: Chloralictus versatus, c, n, Curtisapis coriacea, Seladonia.

Diptera (1)—Syrph.: Mesogramma marginata $\mathrm{f}, \mathrm{n}$.

Lepidoptera (3)-Papition.: Callidryas, Pieris; Hesperi.: Pholisora eatullus.

Hibiscus lasiocarpos (6:270-1).-Blooms July 20-September 16 ; 8 visitors observed, July 29-September 11 ; Ma, W.

Birds (1) -Trochil.: Trochilus.

Long-tongued Bees (6)-Bomb.: Bombias separatus $q$, Bombus americanorum $\delta^{\uparrow} q \not{q}$, ab ; Megachil.: Megachile brevis $\sigma^{\lambda}$; Eucer.: Melissodes bimaculata $\delta^{\lambda}+$; Emphor.: Emphor $\delta^{\pi}+$, se, ab, Melitoma $\sigma^{\pi}$.

Coleoptera (1)-Scarabae.: Euphoria sepulchralis $\mathrm{f}, \mathrm{n}$.

Hibiscus militaris.-Blooms August 9-31; 3 visitors observed, August 15; Ma, R.

Long-tongued Bees (3)-Bomb.: Bombus americanorum $\Varangle ;$ Emphor.: Emphor ơ 오 se, ab, Melitoma ơ.

Hibiscus trionum (int, 6:271-2).-Blooms June 20-October 15 ; 5 visitors observed, August 28-September 26; Ma, Y.

Long-tongued Bees (3)-Bomb.: Bombias auricomus $q, 2$, Bombus americanorum ô $\Varangle, 3 ;$ Eucer.: Melissodes boltoniae $q, 1$.

Lepidoptera (2)-Papilion.: Eurymus 2, Pieris 1. 


\section{Nymphaeaceae}

Castalia tuberosa (2:123-5).-Blooms May 22-September 18; 12 visitors observed, May 22-September 17; Mi, W.

Short-tongued Bees (8)-Halict. ( 9, c) : Agapostemon radiatus ab, 1 dead, viridulus ab, Chloralictus nymphaearum, Curtisapis coriacea dead, Evylaeus nelumbonis ab, Halictus lerouxii dead, parallelus 1 dead; Prosopid.: Prosopis nelumbonis $q$, $f$.

Diptera (3)-Syrph.: Helophilus divisus $f$, ab, latifrons $f$; Bombyli.: Sparnopolius, dead, n.

Coleoptera (1)-Rhipiphor.: Rhipiphorus limbatus, dead.

Nelumbo lutea (2:297-8).-Blooms June 23-August 31; 32 visitors observed, July 26-August 12 ; Mi, W.

Long-tongued Bees (6)-Ap.: Apis e, ab; Bomb. (n) : Bombias scutellaris $\measuredangle$, expl, separatus $\measuredangle$, expl, Bombus americanorum $\Varangle$, expl, impatiens $q$, dead; Ceratin.: Ceratina $q$, c.

Short-tongued Bees (12)-Halict.: Agapostemon radiatus $q$, c, ab, Chloralictus nymphaearum $q$, c, type, pilosus + , c, ab, sparsus $q$, c, versatus $0^{7} q, f, c, a b$, Evylaeus nelumbonis $q, \mathrm{c}, \mathrm{ab}$, type, Halictus lerouxii $q$, c, parallelus $q$, c, ab, Odontalictus $q$, c, Oxystoglossa confusa + , c, ab; Prosopid. ( $(, \mathrm{f})$ : Prosopis nelumbonis, type, ziziae.

Other Hymenoptera (2, expl, n)-Scoli.: Elis interrupta, Scolia bicincta.

Diptera (8)—Syrph. (f) : Mesogramma marginata, Orthoneura nitida, Pipiza pulchella ab, Sphaerophoria, Syritta, Syrphus ribesii; Musc.: Pseudopyrellia, dead, n; Ephydr.: Notiphila f, fq.

Coleoptera (4, f)-Cerambyc.: Typocerus badius; Chrysomel.: Diabrotica 12-punctata ab; Coccinell.: Hippodamea 13-punctata, Megilla.

Nymphaea advena $(2: 122-3)$.-Blooms May 19-August $20 ; 2$ visitors observed, August 20; Mi, Y.

Short-tongued Bees (1)-Halict.: Evylaeus nelumbonis $q$, c.

Diptera (1)—Syrph.: Helophilus divisus.

\section{Oleaceae}

Fraxinus americanus (anemophilous).-One visitor observed, April 19.

Long-tongued Bees (1)-Ap.: Apis e, ab.

Onagracene

- Circaea lutetiana (5:273).-Blooms June 20-July 26; 18 species and 94 individuals, June 25-July $10 ; \mathrm{Mi}$, W. 
Long-tongued Bees $(2: 6)-B o m b$.: Bombus americanorum 8 , 1; Ceratin.: Ceratina 9 , se, 5.

Short-tongued Bees $(9: 86)$ - Malict.: Augorhlora viridula P, se, 1 , Chloralietus obseurus + , se, 1 , sparsus $\$$, 1, versatus ? se, 30, Curtisapis fuscipennis $q$. se, 14, Evylaeus pectinatus 9. se, 1. peetoralis $q, 1,4$-maeulatus of $_{f}, \mathrm{se}, 22$, Oxystoglossa confusa $\&, \mathrm{sp}, 15$.

Other Hymenoptera $(1: 1)$-Chalcid.: Smiora debilis.

Diptera (6:11)—Syrph.: Allograpta 1, Mesogramma geminata 5, marginata 1, Baceha fuseipenuis 1 ; Bombyli. (f, n): Authrax sinuosa 2, Argyramoeba oedipus 1.

Gaura biennis $(5: 273)$.-Blooms July 2-Oetober 24; 7 visitors observed, August 23-September 10; Ma, W.

Long-tongued Bees (5)-1p.: Apis se; Bomb.: Bombus ameri-

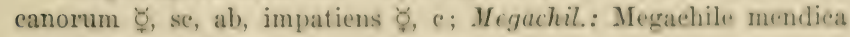
ㅇ, e ; Eucer.: Melissodes bimaculata $q$, se.

Short-tongued Bees (1)-Malict.: Chlomalietus versatus \&, $r, \mathrm{n}$. Diptera (1)—Syrph.: Syrphus americanus $\mathrm{f}, \mathrm{n}$.

Ludvigia alternifolia (5: 271).-Blooms July 11-August 2:3; 17 species and 40 individuals taken on August :3, \&: Mi. Y.

Long-tongued Bees $(3: 4)$-Bomb.: Bombus americanorum $\forall$, 1; Megachil.: Megachile brevis,+ 1 , petulans $\widehat{\delta}, 2$.

Short-tongued Bees (10:32)-Halict. (f): Chloralietus jilosus se, 6, pruinosus 2, sparsus c, 1, versatus se, 13, Halietus parallelus se, 1 , Odontalictus se, 4 , Oxystoglossal confusa 1 , pura 1 , similis se, 1 , Seladonia 2.

Other Hymenoptera (2:2)-Philunth.: Philanthus ventilabris 1; Crabron.: Anacrabro 1.

Diptera (1:1)-Mycetophil.: Sciara atrata 1, n.

Lepidoptera (1:1)-Lycaen.: Everes 1.

Ludvigia polycarpa $(5: 272)$. - Eightern speeies and 59 imdividuals, August $3-19 ;$ Mi (Pol), Y.

Short-tongued Bees $(5: 40)$-Walict.: Chloralietus eressuii ?. 1, versatus ㅇ. se, 36, zephyrus ?, 1, Evylarus peetoralis d, 1, 0xystoglossa similis +1 .

Other Hymenoptera (6:8)-Scoli.: Elis interrupta 1: Phi. lanth.: Cerceris compacta 1: Vesp.: Polistes pallipes 1, variatus 1; Pompril.: Lopghopompilus atrox 2, philadelphious 2.

Diptera (7:11)—Syrph.: Mesogramma marginata 4, polita 1; Tochin.: Archytas analis 1, Belrosia unifaseiata 1, Spallanaania 1; Surophay.: Sareophaca sinuata 2; Anthomy.: Limnophora 1. 
Oenothera biennis $(5: 272)$.-Blooms July 20-October $16 ; 10$ visitors observed, August 13-September 2; Ma, Y.

Birds (1) - Trochil.: Trochilus fq.

Long-tongued Bees (6)-Ap.: Apis se, fq; Bomb.: Bombus americanorum $\delta$ ఫ, sc, fq; Megachil.: Megachile mendica + , c; Eucer.: Anthedon $\delta \uparrow$, sc, ab, oligolege, Epimelissodes obliqua $q$, c, Melissodes bimaculata $q$, c.

Short-tongued Bees $(2, q, \mathrm{e})$-IIalict.: Agapostemon viridulus; Nomi.: Paranomia.

Lepidoptera (1)—Sphing.: Deilephila.

Oenothera fruticosa $(5: 272-3)$.-Blooms May 24-June 29; 28 visitors observed, May 28-June 25; Ma, Y.

Long-tongued Bees (7)-Bomb.: Bombus americanorum + sc,

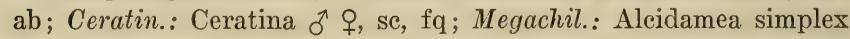
ㅇ, e, Cyphopyga $\delta^{\lambda}+$, se, ab, Megachile brevis $\sigma^{\lambda}+$, Sayapis pollicaris $q$; Eucer.: Tetralonia dilecta $q$, sc.

Short-tongued Bees (9, ㅇ, c, n)-Halict.: Agapostemon viridulus ab, Chloralictus albipennis, versatus, Evylaeus pectoralis, Halictus lerouxii, parallelus, Odontalictus, Oxystoglossa confusa fq, Seladonia.

Diptera (6, f, n)-Syrph.: Eristalis dimidiatus, latifrons, Sphaerophoria, Syrphus americanus, Tropidia mamillata; Tachin.: Cistogaster pallasii.

Lepidoptera (4)-Papilion.: Laertias, Pontia ; Hesperi.: Limochores taumas, Polites fq.

Coleoptera (2, f, n)-Chrysomel.: Diabrotica 12-punctata; Curculion.: Centrinus seutellum-album ab.

\section{OrChidaCeae}

Cypripedium parviflorum pubescens.-Blooms May $7-30 ; 2$ visitors observed, May 15, 17; Ma, Y.

Long-tongued Bees (2, n)-Ceratin.: Ceratina $q$, 1, Zaodontomerus $\sigma^{\lambda}, 1$.

Habenaria leucophaea $(6: 53-4)$.-Blooms June 12-July $12 ; 1$ visitor observed, July 2 ; Ma (S), W.

Lepidoptera (1)—Sphing.: Chaerocampa 1.

Liparis liliifolia.-Blooms May 17-June 20; 2 visitors observed, June $3 ; \mathrm{Mi}, \mathbf{R}$.

Diptera (1)-Anthomy.: Hylemyia juvenalis n.

Hemiptera (1) -Caps.: Lopidea $\mathrm{n}$. 
Orchis spectabilis $(6: 52-3)$.-Bloums May $6-19 ; 2$ visitors observed, May 13; Ma, R.

Long-tongued Bees (2)-Bomb.: Bombias srparatus ?. Bombus americanorum q.

Spiranthes gracilis $(6: 51-2)$.- Bloums August 27 - netober 7 ; 2 visitors observed, September 3,$4 ; \mathrm{Ma}, \mathrm{W}$.

Long-tongued Bees (1)-Bomb.: Bombus amerieanurum of.

Short-tongued Bees (1) -l'anurg.: Calliopsis androniformis?

\section{Orobanchaceae}

Orobanche unifora.-Bluoms April 27-May 28: 1 visitor observed, May $16 ;$ Ma, W.

Long-tongued Bees (1)-Bomb.: Bombus americunorum ?.

\section{Oxalidaceas}

Oxalis corniculata.-Blooms April 14-July $7 ; 18$ visitors oh. served, May 22-June 10; Mi, Y.

Long-tongued Bees $(4)-1 p$. : Apis; Cralin.: Ceratina of $f$; Megachil.: Aleidamea simplex of, Osmia pumila $q$, se.

Short-tongued Bees $(9,+$, se $)$ - Ialict.: Chloralietus albipennis, pilosus fq, pruinosus fq. tegularis, versatus, Erylateus foxii, pertoralis, Odontalietus, Seladonia.

Diptera (1) - Syrph.: Mesogramma marginata sf.

Lepidoptera (4)-Nymphal.: Phyciodes fq; Papilion.: Pioris. Pontia; Hesperi.: Pholisora catullus.

Oxalis stricta.-Blooms June 1-November 7 ; 12 visitors observed, June 18, August 4, 22, October 9; $\mathrm{Mi}, \mathrm{Y}$.

Long-tongued Bees (2)-Bomb.: Bombus anerieanorum 5: Eucer:: Melissodes bimaculata 우.

Short-tongued Bees (2, \&, se)-IIalict.: Chloralietus versatus; Panurg.: Calliopsis andreniformis.

Diptera (5)-Syrph. (f, n) : Mesogramma mareiuata, Syritta: Emprid.: Parempis; Bombyli.: Sparnopolius, Thlipsngauter.

Lepidoptera (3)-P'upilion.: Pieris; Ilsperi.: Pholisura eatul. lus, hayhurstii.

Oxalis violacea $(6: 272-3)$.- Blooms $\Lambda$ pril fi-June 10$): 31$ vis. itors observed, April 23-May 17; Mi (Ma), R.

Long-tongued Bees (12)-Ap.: Apis: Bomb,: Bombias auri. comus \&. Bombus anerieanorum \&: Ceralin.: Ceratina of \&. fif. Zaodontomerus de fq: Megachil.: Monilosmia of, Usmia pumila ?. fq ; Nomad.: Centrias amerieamus of. Holonomada superha 6 8. fiq. 
Nomada cressonii $\sigma^{\lambda}$, parva $\hat{\delta} q$, fq, type; Eucer.: Tetralonia dilecta $\delta$ q, fq.

Short-tongued Bees (14)-Halict. (q): Agapostemon radiatus, viridulus, Chloralictus albipennis, pilosus, se, ab, versatus, Curtisapis forbesii, Evylaeus pectoralis, Halictus lerouxii, sc, ab, Odontalictus se, Oxystoglossa confusa ab, Seladonia sc, ab; Andren.: Iomelissa $q$, Opandrena cressonii $q$, personata $\sigma^{\uparrow}+$.

Diptera (1)—Bombyli.: Bombylius pulchellus.

Lepidoptera (3)-Nymphal.: Phyciodes; Papilion.: Eurymus; Hesperi.: Thanaos brizo.

Coleoptera (1)-Buprest.: Acmaeodera culta f, n.

Papaveraceae

Sanguinaria canadensis $(5: 175-6)$.-Blooms March 16-April 30 ; 11 visitors observed, April 11-14; Mi, W.

Long-tongued Bees (3)-Ap.: Apis e, ab; Ceratin.: Zaodontomerus $\sigma^{\pi}$; Megachil.: Ceratosmia $\delta^{\top}$.

Short-tongued Bees (4)-Halict. () : Chloralictus sparsus, zephyrus, Evylaeus foxii; Andren.: Andrena carlini ơ $q$, fq.

Diptera (3)-Syrph.: Syrphus americanus f; Bombyli.: Bombylius major; Tachin.: Gonia capitata.

Coleoptera (1)-Oedemer.: Asclera ruficollis f, fq.

\section{Passifloraceae}

Passiflora lutea.-Blooms July 5-September 26; 6 visitors observed, July 8-September 9 ; Mi, W.

Short-tongued Bees (4)-Halict. ( $q$, c, n) : Chloralictus pruinosus 1, versatus 2; Collet.: Colletes latitarsis $0 \hat{q}$, ab ; Panurg.: Anthemurgus passiflorae $\delta$, sc, ab, type, oligolege.

Other Hymenoptera (2)-Eumen.: Eumenes fraternus 1; Crabron.: Anacrabro 1.

\section{Pedaltaceae}

Martynia louisiana (5:65).-Blooms July 12-September 14; 1 visitor observed, September $3 ; \mathrm{Ma}, \mathrm{R}$.

Long-tongued Bees (1)—Bomb.: Bombus americanorum $\Varangle$.

\section{Phrymaceae}

Phryma leptostachya $(5: 68)$.-Blooms July 3 -September $3 ; 2$ visitors observed, July 27, August 8; Mi, R.

Long-tongued Bees (1)-Ceratin.: Ceratina 우.

Short-tongued Bees (1)-Halict.: Oxystoglossa confusa 0 .

\section{Phytolaccaceae}

Phytolacca decandra $(5: 68-9)$.-Blooms June 14-October 15; 12 visitors observed, July 17, 23; Mis, W. 
Long-tongued Bees (1) - Ap.: Apis.

Short-tongued Bees (5) - Mulint.: Chloraliesus sparsus of \&, so, ab, versatus $f$, se, ah, aphyrus $f$. Oduntalietus o. Seladonia 6 ?

Other Hymenoptera (2)-Vesp.: Polistes pallipes: Pompril.: Prioenemoides fulvieornis.

Diptera (4)-Siyrh.: Meogranma geminata, Syrita ab: Empid.: Parempis; Tachin.: Arehytas analis.

\section{Plantaginaceaf (anemophilous)}

Plantago major.-Three visitors observed, June 23-July 17.

Long-tongued Bees (1)-Bomb.: Bombus anerieanorum V, $\ulcorner, 1$.

Short-tongued Bees $(2, ?$ e) - Halict.: Chloralietus versatus 11 , Oxystoglossa similis 1 .

\section{Poremoniaceate}

Phlox divaricata $(8: 143,13: 578)$.-Blooms April :-Jume 2; 25 visitors observed, April 25-May 23; Ma, R.

Long-tongued Bees $(8)-B u m b$. ( $(7)$ : Bumbias separatus, Bombus amerieanorum ab, eonsimilis, impatiens, ridingsii, Psithyrus variabilis: Meguchil.: Osmia corlata d; Eucer.: Tetralunia dileeta ơ $q, \mathrm{ab}$.

Diptera (1) - Bombyli.: Bombylius atriceps.

lepidoptera (16) - Nymphal.: Anosia; Paprition.: Euphoisules, Eurymus, Heraclides, Iphiclides, Jasoniades, Laertias, Papilio; Hesperi.: Atrytone zabulon ab, Epargyreus, Limochores taumas, Thanaos icelus, Thorybes bathyllus; Sphing.: Deilephila. Hemaris thysbe; Noctu.: Plusia simplex.

April 29, May 6, 11, 18, 20, 1909, the following 9 speeies and 6: individuals were taken on the flowers:

Long-tongued Bees $(6: 56)-$ Bomb.: Bombias separatus 1, Bombus amerieanorum 7 , consimilis 1 , ridingsii 1 , Psithyrus variabilis 3; Eucer.: T'etralonia dileeta 43.

Lepidoptera (3:6)-Pupition.: Eurymus 1, Iphieliares 1; IIesperi.: Atrytone zabulon 4.

Phlox glaberrima $(8: 142)$.-Blooms May 28-August $6 ; 8$ vis. itors observed, May 28-July 18; Ma, R.

Lepidoptera (7)-Nymphul.: Amosia: Papilion: Eurymus. Heraclides, Laertias. Papilin; IIesperi.: Polites; Clemuch. Serpois.

Diptera (1) -Syrph.: Syrphus amerieanus $\mathrm{f}, \mathrm{n}$.

Phlox pilosa $(8: 142-3)$.-Blooms April 27-June 29: 20 vis. itors observed, May \&-June 8; Ma, R.

long-tongund Beos $(7)$-Bomb. (9): Bombias aurieomus bq, separatus, Bombus americanorum, impatiens: Nomaif.: Holmomacia 
superba $q$; Eucer.: Tetralonia dilecta $\delta$ \% , ab ; Anthophor.: Anthophora ㅇ.

Diptera (1)-Bombyli.: Bombylius atriceps.

Lepidoptera (12)-Nymphal.: Phyciodes, Vanessa huntera; Lycaen.: Chrysophanus; Papilion.: Euphoeades, Eurymus ab, Jasoniades, Papilio; Hesperi.: Epargyreus, Polites fq, Thorybes bathyllus fq, pylades; Noctu.: Plusia simplex fq.

May 16, 1911, 6 species and 48 individuals were taken on the flowers, as follows:

Long-tongued Bees $(2: 21)$-Bomb.: Bombias auricomus 1; Eucer.: Tetralonia dilecta 20.

Lepidoptera (4:27)-Papition.: Eurymus 10; Hesperi.: Epargyreus 2, Polites 8, Thorybes bathyllus 7 .

Polemonium reptans (13:578-9).-Blooms April 12-May 24; 50 visitors observed, April 14-May 20; Ma, R.

Long-tongued Bees (21)-Ap.: Apis sc, ab; Bomb. (q) : Bombias auricomus, separatus $f q$, Bombus americanorum ab, consimilis, impatiens $f q$, ridingsii, Psithyrus variabilis; Ceratin.: Ceratina $\delta$ + , ab, Zaodontomerus $\delta^{\top}$; Megachil.: Alcidamea simplex ô, Ceratosmia $\delta^{\lambda}+$, Diceratosmia $q$, se, fq, Osmia atriventris $\delta$, pumila $\delta$ q, se, ab; Nomad.: Gnathias ovatus $q$, xanthoparius $\hat{\delta}$, fq, Holonomada affabilis $\delta^{\top}$; Eucer.: Tetralonia belfragei $\delta^{\top} \uparrow$, se, ab, dilecta ô, fq; Anthophor.: Anthophora $\sigma^{\top}$.

Short-tongued Bees (17)-Halict. (q): Agapostemon radiatus, Chloralictus coreopsis se, obseurus, pilosus se, versatus, Curtisapis coriacea se, Halictus lerouxii se, Oxystoglossa confusa se, ab, pura se, fq, similis; Andren.: Andrena carlini $q$, geranii $\delta$, fq, nasonii o, e, pruni $q$, sayi $q$, se, Ptilandrena g. maculati $\delta^{\lambda}$, polemonii $\delta^{\lambda}+$, sc, ab, type, oligolege.

Diptera (5)-Syrph.: Mesogramma marginata f, n, Pipiza femoralis f, n, Rhingia sf; Empid.: Pachymeria; Bombyli.: Bombylius major.

Lepidoptera $(5, \mathrm{n})$-Papilion.: Eurymus fq; Hesperi.: Thanaos brizo, juvenalis fq; Sphing.: Hemaris thysbe; Noctu.: Plusia simplex fq.

Coleoptera (2, f, $\mathrm{n})$-Anthic.: Corphyra terminalis ; Coccinell.: Megilla.

April 14, 21, 28, 1910, the following 30 species and 133 individuals were taken on the flowers:

Long-tongued Bees (15:90)-Ap.: Apis 26;Bomb.: Bombias auricomus 3, separatus 6, Bombus americanorum 5, impatiens 5, ridingsii 1 , Psithyrus variabilis 1 ; Ceratin.: Ceratina 3 , Zaodon- 
tomerus 1; Megachil.: feratosmia 1, Dieeratosmia 7, Osmia atriventris 3, pumila 6; Euct.: Tetralnnia beliragei 17, dileeta 5.

Short-tonerued Bees (9:21)-Malicl.: Agaposiomon maliatus 1, Chloralietus coreopsis 1 , pilosns 3, versatus 1, Cumisapis moriarea 2. Oxystuglossa pura 6, similis 2: Andren.: Andrena pruni 1, Ptilandrena polemonii 4.

Diptera (2:2)-Syrph.: Pipiza iomoralis 1: Bombyli.: Bumbylius major 1 .

Lepiduptera (4:20)-P'upilion.: Furymus 5: Ilesperi.: Thamans juvenalis 6; Sphing.: Hemaris thysbe 1; Noctu.: P'lusia simplex 8 .

\section{POLYGALACEAE}

Polygala sanguinea.-Blooms June s-August 27; 4 visitors observed, August 26, 27; Mas, R.

Long-tomgued Bees (2, f)-Eprol.: Triupeolus ercestuii ; Eucer.: Melissodes nivea iq.

Short-tungued Bees (1)-Panurg.: Calliopsis andreniformis $\mathcal{C}$ ㅇ, se, $f$ q.

Diptera (1)-Bombyli.: Systocehus.

\section{Polygonaceate}

Polygonum acre.-Flnoms June 24-Oetober 226; 10 visiturs observed, August 26-September 16; Mis, W.

Long-tongued Bees (1)-Ap.: Apis.

Short-tungued Bees (1) - Maliel.: Augochlora virisula f.

Wther Hymenuptera (5) - Visp.: Polistes pallipes; Eumrn.: Ancistrocerus eapra, Eumenes fratemus: Crubron.: Xestoerabro 6-maculatus; Nysson.: Pseudoplisus divisus.

Diptera (3)-Syrph.: Platychirus quadratus, Syritta: Tarhin.: Archytas aterrima fq.

Polygonum amphibium.-Ploums Anrmst 2--0etuber :20: 1 vis. itor observed, August 30; Mis, IR.

Other Hymenoptera-Vesp.: Vespula germanica.

Polygonum hydropiper.- IBlooms . July 26 - lwober 6 : 2 vivitors observed, September 16 ; Mis, W.

Short-tongued Bees-IIalict.: Halietus lerouxii $q$.

Other Hymenoptera-Vesp.: Polistes variatus.

Diptera-T'achin.: 'Tachina mella.

Polygonum hydropiperoides $(9: 72-3)$. - Itoms July 11 - -

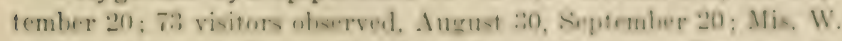

Long-tongued Bees (4) $-A$ p.: Apis; Ceratin.: Ceratina Megachil.: Megachile brevis $\hat{\delta}$, mendica ㅇ. 
Short-tongued Bees (10)-Halict.: Agapostemon radiatus $\hat{\sigma} q$, Curtisapis coriacea $\hat{\delta}, f q$, Halictus lerouxii $q$, Seladonia $q$; Prosopid.: Prosopis pygmaea $\delta$; Collet.: Colletes americanus $\delta^{\lambda} q$, fq, armatus $\delta^{\lambda}$, ab, eulophi $\delta^{\lambda}$, latitarsis $q ;$ Andren.: Pterandrena solidaginis .

Other Hymenoptera (39)-Sphec.: Ammobia ichneumonea, Isodontia apicalis, Priononyx atrata, thomae, Sceliphron; Scoli.: Elis 5-cincta; Philanth.: Cerceris fumipennis, kennicottii, Eucerceris, Philanthus punctatus, ventilabris; Vesp.: Polistes pallipes, variatus; Eumen.: Aneistrocerus capra, Leionotus arvensis, dorsalis; Crabron.: Anacrabro, Synothyreopus, Xestocrabro texanus, trifasciatus; Oxybel.: Notoglossa americana, Oxybelus 4-notatus; Larr.: Astatus unicolor, Larropsis fq, Tachytes aurulentus; Nysson.: Pseudoplisus phaleratus; Pompil.: Batizonus, Ceropales fraterna, Episyron, Lophopompilus philadelphicus, Poecilopompilus interruptus, Priocnemoides fulvicornis; Tiphi.: Tiphia clypeata; Mutill.: Sphaerophthalma; Chrysid.: Chrysis texana, Hedychrum violaceum, wiltii, Holopyga, Tetrachrysis nitidula.

Diptera (16)_Syrph.: Eristalis bastardi, flavipes, Paragus tibialis, Syritta, Tropidia quadrata fq; Bombyli.: Systoechus; Conop.: Conops brachyrrhynchus; Tachin.: Archytas analis, Chaetogaedia analis; Sarcophag.: Helicobia helicis, 4-setosa, Sarcophaga hunteri; Musc.: Compsomyia, Phormia regina, Pseudopyrellia; Anthomy.: Coenosia antica.

Coleoptera (4)-Chrysomel.: Disonycha; Coccinell.: Coccinella 9-notata; Curculion.: Listronotus; Lampyr.: Chauliognathus pennsylvanicus.

Polygonum lapathifolium.-Blooms June 22-September 19; 18 species and 28 individuals observed, August 22, 1910; Mis, W.

Long-tongued Bees (1:1)-Bomb.: Psithyrus variabilis $\delta$, 1 .

Short-tongued Bees (3:3, ᄋ)-Halict.: Augochlora fervida 1, Chloralictus pilosus 1, Halictus parallelus sc, 1 .

Other Hymenoptera $(9: 16)$-Scoli.: Elis interrupta 1, 5-cincta 5, Scolia bicincta 1; Philanth.: Eucerceris 3, Philanthus punctatus 2, ventilabris 1; Vesp.: Polistes variatus 1; Larr.: Astatus unicolor 1; Chrysid.: Hedychrum wiltii 1.

Diptera (3:3)-Tachin.: Gymnosoma 1, Trichophora 1; Anthomy:: Phorbia platura 1.

Lepidoptera (1:1)-Nymphal.: Phyciodes 1.

Hemiptera $(1: 4)$-Lygae.: Lygaeus 4.

Polygonum pennsylvanicum $(9: 72)$.-Blooms June 20-October 26; 87 visitors observed, August 8-September 22; Mis, W. 
Long-tongued Bees $(10)-4$ p.: Apis so, ab: Ismb.: Bombias separatus of, Bombus amerieanoram of 8 , ah, impatiens of $\%$, ah: Meguchil.: Megachile brevis of $q$, petulaus of ; Eucul.: Epeolus pusil-

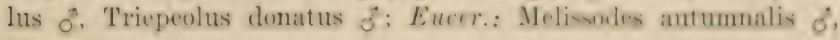
bimaculata $q$.

Short-tongued Bees (11)-Maliet: Aeapostruon raliatus of, Augochlora viridula o, Chlomalietus pilosus \&. sparsus q. rersatus

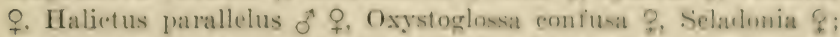
Audren.: Pteramblrena asteris o, solidaginis ?: Panury.: Calliopsis andreniformis $\hat{\delta}$ 요 fq.

Other Hymenoptera (29)_óphe.: ('hlorion, Priononyx atmata, Sphex nigricans; Bembic.: Biryrtes rentralis: Scoli.: Elis 5-cineta i1. Seolia bieineta; Philunth.: Cereris elypeata, fulvipes, Euerceris: Vesp.: Polistes annularis, pallipes, rubiginosus, variatus, Vespula cumeata fq, erermaniea ab: Eumen.: Aneist rocerus eampes. tris, eapra fu, tigris fq; Crubron.: Solenius, Xestoerahro 6-maculatus: Larr.: Larropsis, Taehytes aurulentus, chongatus: Pompil.: Anoplius illinoensis, Lophopompilus philarelphicus, Planierps niger, Pompiloides amerieanus, Priocnemoides fulvieornis; Ichneumon.: Ceratosoma.

Diptera (2s)-Syrph.: Eristalis aeneus, tenax, transwersus, Mesogramma marginata, polita, Milesia, Sphacrophoria, Syritta, Syrphus amerieanus id, torvus, Tropidia quadrata; Bombyli.: Sparnopolius; Tachin.: Archytas analis, aterrima ab, Pelvosia unifasciata, Chaetogaedia analis, Cistogaster pallasii, Linnaemyia. Paradidyma, Senotainia rubriventris, Siphoplacria anomala, Spallanzania; Sarcophag.: Helicobia 4-setosa, Sarophlata cimbieis: Musc: Compsomyia, Graphomyia, Phormia regina, Psemlopyrellia.

Lepidoptera (8) - Vymphal.: Vanessa atalinta: Lycatn.: Chrysophanus; Papition.: Eurymus, Pieris, Pontia: IIsperi.: Limochores taumas; C'tenuch.: Seepsis; Noctu.: Helinthis armiger.

Coleoptera (1)-Lampyr.: Chauliognathus pennsylvanious.

Angust 29, 1910, the following 11 speeies and 22 indiviluals were taken on the flowers:

Long-tongued Bees $(3: 10)-4$ p.: Apis 6; Megarhil.: Megarhile brevis 3 , petulans 1 .

Short-tongued Bees (1:2)-IIalict.: Haliotus parallelus 2.

Other Hymenoptera $(4: 7)$-Bembie, Bieyrtes ventralis 1 ; Scoli.: Elis 5-cineta 4; Philunth.: Eucereeris 1: Ichneumen.: Ceratosoma 1.

Diptera (3:3)-Syrph.: Sphacrophoria 1; Tachin.: Semotainia rubriventris 1 , Spallanzania 1. 
Polygonum persicaria (int).-Blooms May 10-October 26; 13 visitors observed, August 4-September 15; Mis, W.

Long-tongued Bees (1)-Bomb.: Bombus impatiens ô ఫ్ .

Short-tongued Bees (4)-Halict.: Chloralietus sparsus $\delta$ 의, se, $f q$, versatus $q$, fq, zephyrus $q$, Halietus lerouxii $\delta$.

Other Hymenoptera (4)-Philanth.: Cerceris finitima; Vesp.: Polistes annularis; Crabron.: Solenius; Oxybel.: Notoglossa frontalis.

Diptera (3)-Syrph.: Syritta ab; Bombyli.: Anthrax alternata f; Anthomy.: Phorbia acra.

Lepidoptera (1)-Papition.: Pontia.

Polygonum scandens.-Blooms July 10-October 1; 64 species and 395 individuals, August 22-September 15; Mis, W.

Long-tongued Bees (2:5)-Ceratin.: Ceratina + , 1 ; Nomad.: Nomada vicina $\delta^{\lambda}, 4$.

Short-tongued Bees (10:169)-Hatict.: Chloralictus sparsus ${ }^{\top}$, 144, versatus $\delta+\%, 3$, zephyrus $\delta, 9$; Sphecodini: Machaeris stygia $\delta, 3$; Prosopid.: Prosopis pygmaea $\delta, 1$, saniculae $\delta, 1$, ziziae $q, 1$; Collet.: Colletes americanus 01,1 ; Andren.: Pterandrena solidaginis , 1 ; Panurg.: Pseudopanurgus compositarum $\delta$ $~+5$.

Other Hymenoptera (22: 42)—Sphec.: Sphex gracilis 2, vulgaris 1 ; Philanth.: Cerceris finitima 1 , fumipennis 1 , Philanthus punctatus 1; Vesp.: Vespula germanica 1; Eumen.: Ancistrocerus tigris 2, Eumenes fraternus 1, Leionotus perennis 1, scrophulariae 3; Crabron.: Anacrabro 1, Lindenius errans 2, Rhopalum scutellatum 1; Oxybel.: Notoglossa americana 2, frontalis 4; Larr.: Astatus bicolor 1, Larropsis 3; Pompil.: Lophopompilus atrox 1; Chrysid.: Hedychrum violaceum 2; Chalcid.: Leucospis 1, Perilampus cyaneus 2 , platygaster 8 .

Diptera (25:74)-Mycetophil.: Sciara atrata 19; Syrph.: Milesia 3, Syritta 2; Tachin.: Actia 2, Archytas analis 1, aterrima 3, Cyphocera 4, Exorista confinis 1, Hypostena dunningii 1, Linnaemyia 1, Pachyophthalmus signatus 1 , Paradidyma 1 , Senotainia trilineata 5, Siphoplagia flaccidirostris 1 , Sturmia phyciodis 1 , Trichopoda pennipes 7, Trichophora 1, Xanthomelana 2; Sarcophag.: Helicobia helicis 5, Sarcophaga assidua 5, eimbicis 3; Musc.: Lucilia eaesar 1, Musca 1, Pseudopyrellia 2; Ephydr.: Ochthera, pred, $\mathbf{n}, 1$.

Lepidoptera (4:4)-Nymphal.: Vanessa atalanta 1; Lycaen.: Everes 1; Papilion.: Pieris 1; Hesperi.: Limochores taumas 1.

Coleoptera (1:1) -Lampyr.: Chauliognathus pennsylvanicus 1 . 


\section{Pontedemacear.}

Pontederia cordata.- Bloms July 1 - Septemher 15; 2 visitors observed, August 8, September 15; Ma (Mas), R.

Long-tongued Bees (1)-Eucer.: F'lorilegus of.

Lepidoptera (1)-Papilion.: Eurymus.

Portulacaceaz.

Claytonia virginica $2: 177-4)$. - Elooms March 16-May 24: 103 visitors observed, April 8-May 11; Mi, W.

long-tongued Bers (21) - Apr: Apis se, ab; Bonb. (9) : Isumbias auricomus. Bombus amerieanorum, ensimilis, impatiens: Cratin.: Ceratina of 9. Zaodontomerus o: Megachil.: Ceratemia of 8. Fi, Osmia at riventris of pumila $\mathcal{E}$ \&: Nomul.: Tnathias euneatus $\mathcal{S}$.

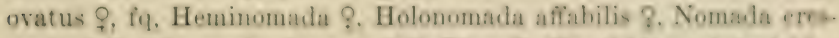

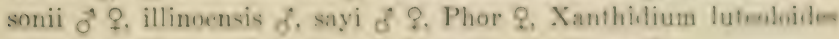
$\delta$ o type; Eucer.: Tetralonia belfragei of $q$, dilecta $q$.

Short-tongued Bees (37)-Llalict. (8): Agapostemon ratlatus se. splendens, Augochlora viriduln, Chloralietus ermosonii, illinuensis, pilosns, sparsus, versatus se. $\mathcal{f}$, zephyrus, Curtisapis eoriaces se. Evylueus areuatus, foxii, peetoralis, truncatus, Halietus lerwuxii se, ab, Odontalietus, Oxystuglosea confusa dy, type, similis, Sela. donia se, ab; Spheerolini : Sphecenles arvensis: Collet.: Colletes inasequalis of q: Andren.: Andrena arabis ?. carlini of $q$, fu. dun.

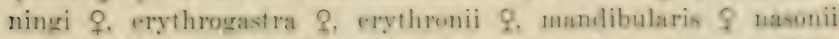
Q. pruni Q. sayi $\delta$ Q. Opandrena cresonii $\delta$ Q se. iq. miserabilis $d$ q. se. fq. personata $\delta$, iq. Ptilandrena erigeniae $d$ \&. se, ab,

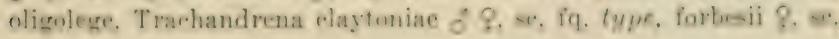
fq, rugosa $\delta$.

Diptera (35)-Syrph.: Allograpta, Eristalis dimiliatus, lati. frons. Ifelophilus similis. Melanostoma chesurum. Mesorramms geminata. marginata. Paragus bicolor fq. Rhingia, Sphaerophoria, Syritta, Syrphus amerimanus, arenatus, ribesii, torvus: Limpiel.: Empis humilis, nuda, wiosa: Bumbyli.: Bombylins major fą: Conop.: Myopa sesirulosa: Sarcophag.: Calliphora erythroepghala, Cynomyia, Helicolia lielieis, 4-setosa, Sareoghana sinuata: Mfoke: Lueilia sylvarum, Phormia reina. Psoudopyrellis; Anthmay: Comosia anties, Limnophors. Phorhis sem. platurs: Scatophay, Seatophaga; Seps.: Prochyliza, Sepsis.

Lepidaptera (9)-Nymphal.: Phyciodes. Vanees abalana: Lycaen.: Everes: Papilion.: Furymus, Iphielides. Pieris. Poutis: IIesperi.: Thanas icelus, juvenalis.

Coleoptera (1)-Coccinell.: Megilla i, $n$. 
May 5, 6, 9, 10, 1916, the first 100 individuals, representing 27 species, taken on the flowers are as follows:

Long-tongued Bees (4:10)-Ap.: Apis 4; Bomb.: Bombus consimilis 1; Megachil.: Osmia pumila 2; Nomad.: Gnathias ovatus 3.

Short-tongued Bees (15:81) - Halict.: Chloralictus pilosus 5, sparsus 3, versatus 5, zephyrus 2, Evylaeus foxii 1, truncatus 1, Odontalictus 5; Andren.: Andrena arabis 1, nasonii 3, Opandrena cressonii 2, miserabilis 3 , personata 5 , Ptilandrena erigeniae 39; Trachandrena claytoniae 2, forbesii 4.

Diptera (8: 9)-Syrph.: Mesogramma geminata 1; Conop.: Myopa vesiculosa 1; Sarcophag.: Helicobia helicis 1, Sarcophaga sinuata 1; Musc.: Lucilia sylvarum 2; Anthomy.: Limnophora 1, Phorbia acra 1, platura 1 .

Portulaca oleracea (int).-Blooms June 12-October $3 ; 3$ visitors observed, August 11; Mi, Y.

Short-tongued Bees (1)-Panurg.: Calliopsis andreniformis $q$.

Diptera (1)—Syrph.: Syritta.

Coleoptera (1)-Malach.: Collops.

\section{Primulaceae}

Dodecatheon meadia (8: 104-6).-Blooms April 23-May 30; 9 species and 38 individuals observed, May 2-23; Ma, W.

Long-tongued Bees $(6: 35)-B o m b$. (q): Bombias auricomus 4, separatus 4, Bombus americanorum 21, impatiens 2; Eucer.: Tetralonia dilecta $\delta^{\lambda}, 3 ;$ Anthophor.: Anthophora + , se, 1.

Short-tongued Bees $(2: 2$, $q, \mathrm{c}, \mathrm{n})$-Halict.: Augochlora viridula 1, Oxystoglossa confusa 1.

Lepidoptera (1)-Papilion.: Eurymus 1.

Samolus floribundus.-Blooms June 15-October 8; 1 visitor observed, September 9 ; Mi, W.

Diptera (1)-Empid.: Parempis 1.

Steironema ciliatum (8:106-8).-Blooms June 11-August 6; 2 visitors observed, July 1, 16, 18; Mi, Y.

Short-tongued Bees (2:8)-Halict.: Chloralictus versatus + , cf, 1; Macropid.: Macropis 07 , ef, 7.

Steironema lanceolatum $(6: 47)$.-Blooms June 10-July $15 ; 1$ visitor observed, June 12-July 1; Mi, Y.

Short-tongued Bees (1)-Macropid.: Macropis steironematis $\delta$ o, ef, ab, type.

Steironema quadriflorum (6:48).-Blooms June 4-August 23; 2 visitors observed, July 6,$10 ; \mathrm{Mi}, \mathrm{Y}$. 
Short-tongued Bees $(2, f, e)$-IIalict.: Chloralictus versatus: Macropid.: Macropis, ab.

\section{Ranuncolaceae}

Actea alba $(10: 229)$,-Blooms May 3-23; 3 vicitors olserves!. May 8,$9 ; \mathrm{Mi}, \mathrm{W}$.

Short-tongued Bees (3, 9)-Malict.: Chloraliutus zephyrus c, Evylaeus pectoralis e, ab, Oxystoglossa confusa f.

Anemonella thalictroides.-Blooms April 2-May 2.2 : 21 vicitors observed, April 2-28; Mi, W.

Long-tongued Bees (5)-Ap.: Apis; Crrutin.: Ceratina 6 ? in cop; Meguchil.: Dieeratosmia o. se, Osmia pumila \&. c; Nomud.: Gnathias ovatus ?.

Short-tongued Bees (11)-Malict. (f) : Chlnralietus sparsus sf, versatus sf. fq. Curtisapis coriacea, Evylacus peetoralis, IIalictus lerouxii, Oxystoglossa eonfusa sf, Seladonia sf, fq ; Collet.: Colletres inaequalis os; Andren.: Andreua carlini ${ }^{\circ}$ Opandrena eressunii $q$. Trachandrena claytoniae $\delta$.

Diptera (4) - Syrph.: Brachypalpus frontosus, Eristalis dimidiatus, Sphaerophoria f: Bombyli.: Bombylius major fq.

Colcoptera (1)-Nitidul.: Cereus fq.

Aquilegia canadensis.-Bloms April 30-July 5: 1 visitor observed, May 12; Ma (O), R.

Birds (1)-Trochil.: Trochilus.

Clematis pitcheri $(7: 105)$.-Blooms June 3-July $31: 4$ visitors observed, June 22-July 7 ; Ma, R.

Long-tongued Bees (3)-Bomb.: Bombias auriomus 8 . se, Bombus americanorum $q \not$, se, ensimilis $q$.

Diptera (1) -Syrph.: Volucella vesiculosa.

Clematis virginiana $(7: 10: 3-5)$.-Blooms June 2:-August 16 ; 60 visitors observed, July 21-30; Pol (Mis), W.

On staminate flowers:-

Long-tongued Bees $(2)-A$ p.: Apis; Bomb.: Bombus impatiens ఫ̧, se.

Short-tongued Bers (9)-IIolict.: Agapostemon radiatus of Chloralietus illinowsis $\delta$, fq, sparsus of $P$, se, ah, versatus $\mathscr{Q} ?$, se,

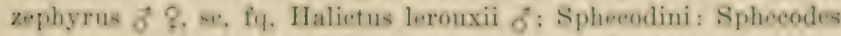

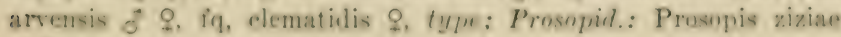
ơㅇ.

Other Hymenoptera (10)-Sphec: Ammobia ichneumonea, Isorlontia apicalis, Seeliphron; Scoli.: Seolin bicineta; Eumen.: 
Ancistrocerus tigris, Leionotus foraminatus; Crabron.: Lindenius errans, Solenius; Oxybel.: Oxybelus 4-notatus; Nysson.: Nysson plagiatus.

Diptera (37)-Stratiomy.: Stratiomyia unilimbata; Syrph.: Allograpta, Eristalis transversus, Mesogramma marginata, Platychirus quadratus, Sphaerophoria, Syritta ab; Conop.: Oncomyia; Tachin.: Archytas analis, aterrima, Belvosia unifasciata, Linnaemyia, Ennyomma globosa, Pachyophthalmus signatus fq, Phorantha purpurascens, Senotainia trilineata ab, Siphona, Sturmia phyciodis; Sarcophag.: Calliphora erythrocephala, Helicobia helicis; Musc.: Compsomyia ab, Graphomyia, Lucilia sericata, Musea ab, Protocalliphora, Pseudopyrellia ; Anthomy.: Calythea, Homalomyia canicularis, prostrata, Limnophora, Phorbia acra fq, platura; Agromyz.: Lobioptera; Seps.: Nemopoda, Piophila, Prochyliza, Sepsis.

Lepidoptera (1)-Lycaen.: Cyaniris.

Hemiptera (1)-Caps.: Lopidea.

Delphinium tricorne $(8: 120-2)$.-Blooms April 23-June 7; 21 visitors observed, May 4-15.

Birds (1)-Trochil.: Trochilus.

Long-tongued Bees (9)-Bomb. (q): Bombias auricomus ab, separatus, Bombus americanorum ab, consimilis, impatiens; Ceratin.: Ceratina $9, \mathrm{n}$; Eucer.: Tetralonia dilecta $\delta^{\lambda}$ ㅇ, se, ab, in cop; Anthophor:: Anthemoessa of, Anthophora + , se.

Short-tongued Bees $(2$, o, c, n) - Halict.: Agapostemon radiatus, Halictus lerouxii.

Lepidoptera (8, n)-Nymphal.: Anosia; Papilion.: Euphoeades, Eurymus, Jasoniades, Papilio; Hesperi.: Atrytone zabulon, Epargyreus; Sphing.: Deilephila.

Hepatica acutiloba (16:153).--Blooms March 18-April 27; 18 visitors observed, March 27-April $19 ; \mathrm{Mi}, \mathrm{W}$.

Long-tongued Bees (2)-Ap.: Apis sc, fq; Ceratin.: Zaodontomerus 07 .

Short-tongued Bees (9)-Halict.: Chloralictus sparsus $q$, versatus 9 ; Collet.: Colletes inaequalis $\delta^{\lambda}$; Andren.: Andrena carlini $\delta$, erythronii $\delta$, mandibularis $\delta$, tridens $\delta$, type, Opandrena miserabilis $q$, Trachandrena rugosa $\delta$, $f q$.

Diptera (7)—Syrph.: Brachypalpus frontosus sf, fq, Eristalis dimidiatus sf, Syrphus americanus f; Bombyli.: Bombylius major fq ; Tachin.: Gonia capitata ; Musc.: Pseudopyrellia fq ; Anthomy.: Phorbia platura. 
Isopyrum biternatum $(5: 173)$ - Blooms Mareh 20-May 2: 62 visitors observed, March 26-April 30 ; Mi, W.

Long-tongued Bees (7)-Ap.: Apis se, fiq; Bomb.: Bombus americanorum q: Ceratin.: Ceratina \&, Zaodontomerns of: Mrge. chil.: Osmia pumila of $q$; Nomad.: Nomada sayi $\{9$; Eucer.: Trtra. lonia belfragei of ?

Short-tongued Bees (25)-Molict. (8): Agapostemon radiatus, Chloralietus eaeruleus, eressnii, obseurus, pilosus, sparsus sp, ver. satus se, ab, zephyrus ab. Curtisapis coriacea, forbenii, Evylaeus foxii, pectoralis, 4-maculatus, Halietus lerouxii so, Oxyatogloma eonfusa se, fq, pura, Seladonia se, ab; Collet.: Colletes inargunlis d: Andren.: Andrena earlini of \&. fq. sayi $f$ \&. fy. Opandrona

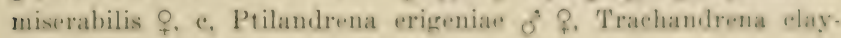
toniae $\delta^{\lambda}$, forbesii $q$, rugosa $\hat{\delta} q$, fq.

Other Hymenoptera (1)-Sapyg.: Sapyga.

Diptera (14) - Syrph.: Chilosia capillata sf. Eristalis dimiliatus, Helophilus similis, Melanostoma obseurum, Mesngramena gemi. nata, marginata $\mathrm{f}, \mathrm{n}$, Sphaerophoria $\mathrm{f}, \mathrm{n}$, Syrphus americanus sf, ribesii, Xylota fraudulosa; Empid.: Empis nuda ; Bombyli.: Bembylius major ab; Tuehin.: Gonia eapitata; Muse.: Pserudopyrellia.

Coleoptera (4, $\mathrm{f}, \mathrm{n})$-Anthic.: Corphyra terminalis; Chrysomel: Diabrotica vittata; Coccinell.: Megilla; Ocdemer:: Aselera rufi. collis.

Hemiptera (1)-Caps.: Lygus.

Myosurus minimus.-Plooms April 7-May 17; 1 visitor wh. served, Mray 8; Mi, Y.

Short-tongued Bees (1)-IIalict.: Chloralietus zephyrus \&. 1.

Ranunculus abortivus (7:108-9).-Blooms Mareh 81-Jume 9: 24 visitors observed, April 8-May 11; Mi, Y.

Long-tongued Bees (4)-Ap.: Apis; Bomb.: Pumbus atneri. canorum \&; Ceratin.: Ceratina d; Nomud.: Gnatlias ovatus ?.

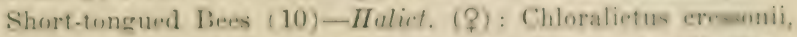
pilosus, sparsus se, fq, versatus, zephyrus, Evylarus peetoralis, 1 sy. stoglosa ennfusa, similis, Seladonia; Andren.: Gpandrena personata $\delta$, f(q.

Diptera (B) $\rightarrow$ syrph.: Chilesia petulea, Mesogramma maryiuata fq. Paragus bienlor $\mathrm{fq}$. Pipiza nigribarba, Sphaceptioria fiq. Turhin.: Apinops, Siphona fq: Surcophag.: Helieobia heliris

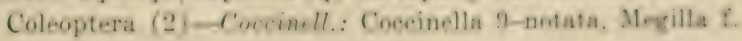

Ranunculus fascicularis $(7: 107-8)$. - Howms Mareh 21-May $20 ; 45$ visitors observed, April 11-May 5; Mi, Y. 
Long-tongued Bees (9)-Ap. Apis; Ceratin.: Ceratina $0^{1} q, \mathrm{fq}$, in cop, Zaodontomerus $\delta^{\tau}$; Megachil.: Osmia illinoensis $\delta$, type,

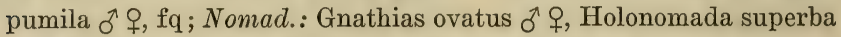
$\delta^{\top}+$, fq, Nomada sayi $\delta^{\top}$; Eucer.: Tetralonia dilecta $\delta^{\top}$.

Short-tongued Bees (15)-Halict. (q) : Agapostemon radiatus se, fq, Chloralictus pilosus, pruinosus se, sparsus, versatus sc, fq, Curtisapis coriacea, Evylaeus pectoralis se, Halictus lerouxii, Odontalictus se, Oxystoglossa confusa se, fq, Paralictus simplex type, Seladonia se ; Andren.: Iomelissa on, Opandrena cressonii $q$, se, miserabilis $\sigma^{\top}$.

Diptera (18)—Syrph.: Chilosia capillata, Eristalis transversus f, Melanostoma mellinum, Mesogramma geminata fq, marginata sf, $\mathrm{fq}$, Orthoneura pictipennis, Paragus bicolor, Pipiza femoralis $\mathrm{fq}$, Sphaerophoria sf, Syrphus americanus, arcuatus, Xylota fraudulosa; Bombyli.: Bombylius major; Tachin.: Gonia capitata; Sarcophag.: Sarcophaga cimbicis; Musc.: Pseudopyrellia; Anthomy.: Coenosia antica, Phorbia acra fq.

Lepidoptera (2)-Papilion.: Eurymus; Hesperi.: Thanaos juvenalis.

Coleoptera (1)-Oedemer.: Asclera ruficollis $\mathrm{f}, \mathrm{n}$.

Ranunculus recurvatus.-Blooms April 30-May 21; 5 visitors observed, May 10, 11, Mi, Y.

Long-tongued Bees (1)-Nomad.: Nomada parva $0^{1}$ ㅇ․

Short-tongued Bees (4)-Halict. (q): Chloralictus obscurus, Oxystoglossa confusa, similis ; Andren.: Opandrena cressonii $\delta$.

Ranunculus septentrionalis $(7: 105-7)$.-Blooms April 8-May 24; 52 visitors observed, April 12-May 7; Mi, Y.

Long-tongued Bees (7)-Ceratin.: Ceratina $\delta^{\lambda} q$, sc, ab, Zaodontomerus $\delta^{\lambda}$; Megachil.: Ceratosmia + , se, Diceratosmia + , c, Monilosmia $\sigma^{\lambda}$, Osmia pumila $\sigma^{\star} \rho, \mathrm{sc}, \mathrm{ab} ;$ Eucer.: Tetralonia belfragei $\delta^{\lambda}$.

Short-tongued Bees (16)-Halict. (q): Chloralictus obscurus sc, fq, pilosus se, sparsus, zephyrus, Curtisapis coriacea se, fq, Evylaeus pectoralis se, 4-maculatus fq, Halictus lerouxii se, Odontalictus se, Oxystoglossa confusa sc, ab, pura, Seladonia sc; Sphecodini: Proteraner ranunculi ô, type; Andren.: Opandrena cressonii $q$, sc, personata $\delta^{\lambda}$, Ptilandrena polemonii $\delta^{\wedge} q$, ab.

Diptera (19)—Syrph.: Chilosia capillata ab, Melanostoma obscurum, Mesogramma geminata, Pipiza femoralis, nigribarba, Sphaerophoria, Syrphus americanus, arcuatus, ribesii, torvus; Bombyli.: Bombylius major, pulchellus; Tachin.: Siphona; Musc.: Morellia; Anthomy.: Anthomyia radicum, pluvialis, Hylemyia dilatata $\mathrm{fq}$, Mydaea diruta, Phorbia platura. 
Lepidoptera (31-Hesperi.: Thanaces brizo, jusemalis, Thery hess bathyllus.

Coleoptera (7)-Anthie.: Corphyma terminalis sf; Carah.: Lebia n: Chrysomel.: Diabrotica vittata f. n: Coccinell: Megilla f, $n$; (curculion. in) : Ceutrinus prrseillus f. Thiogtethus subealvus: Oedemer.: Asclera rufieollis $\mathrm{f}, \mathrm{n}$.

Thalictrum dasycarpum (anemophilous). - One visitor observed, June 3.

Long-tongued Bees (1)-A p.: $\Lambda$ pis $\mathrm{e}, \mathrm{fq}$.

\section{RHAMNACEAE}

Ceanothus americanus $(2: 303-4)$. - Bloorns May 27-July 2; 121 visitors observed, June 16-July 4; Pol, W.

Long-tongued Bees (6)-Bomb.: Pombias auricomus o. e, separatus $q \not$, se ; Cerutin.: Ceratina of; Meguchil.: Nentrypetes irun-

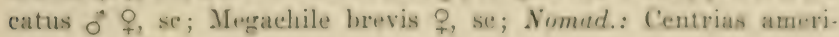
eanus ㅇ.

Short-tongued Bees (18)-Halict.: Chloralietus sparsus o. s.

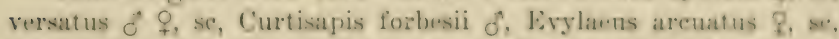
type, pectoralis o. se, truncatus $q$, se. Oxystoglossa confusa f. se. Seladonia f. se; Spheeodini: Spheredes minor $\$$. Sphecolium eressonii of $q$; Prosopud.: Prosupis pygmaea of q, zixiar of q; Collet.: Colletes eulophi $\mathcal{C}^{\circ}$, willistonii $\& ;$ Andren.: Andrena platyparia \&.

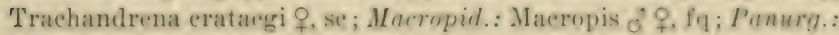
Calliopsis andreniformis $\delta^{\top}$.

Other Hymenoptera (35)-Sphec.: Isodontia apiealis. Soeliphron, Sphex nigricans; Scoli.: Elis 5-eincta; Philanth.: Cereeris elypeata, eompacta, enmpar, finitima, robertsonii, rufinoda; Vesp.: Polistes variatus; Eumen.: Ancistrocerus tigris, Eumenes fraternus. Leionotus dorsalis, foraminatus, fulvipes, fundatus, illinesnsis, ziziae; Crubron.: Solenius; Oxybel.: Notoglosa amerieana, Proulalis, Oxybelus packardii. 4-notatus; Larr. : Tachysphex acutus, belfrarei : Pompil.: Anoplius illinoensis, Ceropales robinsonii, Poneilopompilus navus, Pompiloides marginatus, Psammochares relativus, tenelow. sus; Chrysid.: Hedyehrum violaceum; Chalcil.: Leueospis: Tenthredin.: Hylotoma humeralis.

Diptera (42)-Mycetophil.: Sciara atrata; Stratimy: Xenut.lus: Syrph.: Allograpta, Mesugramma geminata, maryinata, Orthoneura nitida, Parasus bieolor, Sphaerophoria, Syritta, Syr. phus americanus, Tropidia mamillnta, quadrata; Emyral: Par. empis: Bombyli: Anthrax simuosa: Conop.: Conops braebyr. rhymehus, Oncomyia, Stylogaster biemulata, Zolion folvifous: 
Tachin.: Archytas analis, aterrima, Cistogaster occidua, Exorista confinis, Linnaemyia, Ocyptera tenuis, Pachyophthalmus signatus, Paradidyma, Phorantha purpurascens, Siphona, Spallanzania, Trichophora, Xanthomelana; Sarcophag.: Helicobia helicis, Sarcophaga sinuata; Musc.: Lucilia caesar, Morellia, Musca, Phormia regina, Pseudopyrellia; Anthomy.: Limnophora, Phorbia acra; Seps.: Sepsis; Trypet.: Trypeta humilis.

Lepidoptera (2)-Lycaen.: Thecla calanus, sylvinus.

Coleoptera (14)-Cerambyc.: Typocerus sinuatus; Chrysomel.: Diabrotica atripennis, 12-punctata, Pachybrachys; Curculion.: Centrinites, Centrinus picumnus, scutellum-album; Dermest.: Cryptorhopalum haemorrhoidale; Elater.: Limonius; Lampyr.: Telephorus scitulus; Malach.: Attalus; Mordell.: Mordella marginata; Oedemer.: Oxacis; Scarabae.: Trichius piger.

Hemiptera (4)-Caps.: Calocoris, Lygus ; Cyan.: Canthophorus ; Lygae.: Lygaeus.

Rhamnus lanceolata $(9: 157-9)$.-Blooms April 23-May 14; 51 visitors observed on May 14 ; Mis, Y.

Long-tongued Bees (4)-Ap.: Apis; Bomb.: Bombus americanorum $q$; Ceratin.: Ceratina $\delta$; Nomad.: Gnathias euneatus $q$.

Short-tongued Bees (23)-Halict. (q): Agapostemon radiatus fq, Augochlora viridula, Chloralictus illinoensis, pilosus se, fq, pruinosus se, sparsus se, ab, versatus se, fq, zephyrus se, Curtisapis forbesii se, Evylaeus areuatus se, foxii se, fq, Halictus lerouxii se, Oxystoglossa confusa se, fq, pura sc, Seladonia se, ab; Sphecodini : Sphecodium cressonii ; Andren.: Andrena erythrogastra ô, mandibularis $q$, nasonii $q$, se, type, Opandrena eressonii $\delta^{\lambda}+$, sc, ab, miserabilis $\delta$, personata $\delta$ o $q, \mathrm{sc}, \mathrm{fq}$, Trachandrena erataegi $\delta$.

Other Hymenoptera (3)-Eumen.: Ancistrocerus tigris, Eumenes fraternus; Tenthredin.: Dolerus arvensis.

Diptera (21) - Syrph.: Allograpta fq, Helophilus similis, Mesogramma geminata ab, marginata, Orthoneura nitida, Pipiza femoralis, Sphaerophoria fq, Syritta, Syrphus americanus, ribesii, Xanthogramma felix; Empid.: Rhamphomyia priapulus; T'achin.: Peleteria; Sarcophag.: Cynomyia, Sarcophaga cimbicis, sinuata; Musc.: Lucilia eaesar, sericata; Anthomy.: Phorbia acra fq, platura fq ; Scatophag.: Scatophaga.

Rosaceae

Agrimonia striata.-Blooms July 3-September 7; 1 visitor observed, July 24 ; Mi, Y.

Short-tongued Bees (1)_Halict.: Chloralictus versatus + , sc. 


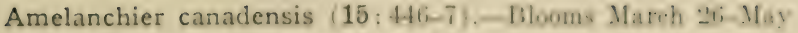
$1 ; 51$ visitors observed, April 10-22; Mis, W.

Long-tongued Bees (6) $-A$ p.: Apis se, ab: Bomb: Bombin

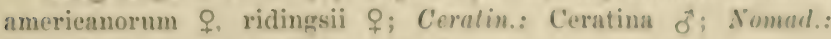
Nomada denticulata 0 , illinoensis of.

Short-tongued Bees (27)-Malict. (P): Agugnstomen tadietus. Chloralietus eacruleus type, eressonii, pilosus so, fa, spanous. versatus se, ah, zephyrus ah, Evylaens arenatus so, P. foril, Ilalintus lerouxii, Seladonia se ; Collel.: Culletes inaequalis of P. Pa: Anderne: Andrena earlini $\delta$ \% erythrogastra $\delta \&$. fq. erythronii $\delta$. man.

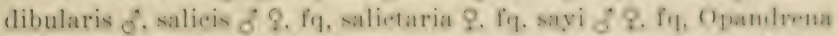

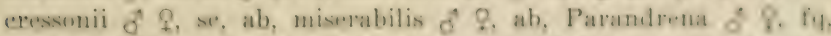
Trachandrena elaytoniae $\delta$ 9. fig. forbesii of ?. fq, tyme, hippotes $\delta \mathcal{*}+$, mariae $\delta^{7}$, rugosa $\delta^{*}$.

Other Hymenoptera (1) - Tenthredin.: Dolerus arvensis.

Diptera (17) $\rightarrow$ Syrph.: Brachypalpus frontosus, Eristalin aeneus, dimidiatus $\mathrm{fq}$, transversus, Helophilus similis, Meneramma geminata, marginata, Platyehirus quadratus, Sphaerofduria. Syrphus amerieanus fy: Bombyli.: Bombylius major: Tochin.: Euphoroera daripennis. Gonia eapitata: Norcophag: Cynonyia fin; Musc.: Psomdopyrellia; Anthomy.: Hammonyia; Seatophay.: Seatophaga $\mathrm{f} q$.

Aruncus sylvester $(15: 437 ; 16: 16.5-9)$.-Bluoms . May 24-June 20; 78 visitors observed, June $4-20 ;$ Mis, W.

On staminate flowers (43):-

Long-tongued Bees (3)-Megurhil.: Neotrypeters truncatus of: Nomad.: Ceutrias amerieanus 9 . Holonomada affabilis $\overrightarrow{6}$.

Short-tongued Bees (6)-Ilulict: Chloralietus versatus 8:

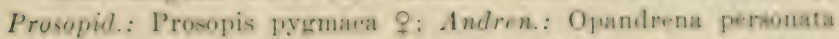
9. sorntina d. Trachandrena uula P. se, spiraeana \&, sc, ab, two , oligolege.

Other Ifymenoptera (3)-Eumen.: Eumenes frateruus: Crobron.: Anacrabro; Oxybel.: Notoglossa frontalis.

Dipura $(7) \rightarrow$ Syrph.: Chilosia pallipes, Chryogesaser nigriges. Spherina rufiventris; Empid.: Empis distans fy: Conep.: Zodion nanellum; Agromye.: Phytonyza palpalis type; Oscin.: Siphonella einerea.

Coleoptera (23)-Bruch.: Bruchus hibisci; Cerambyc: A.maeops direota. I.eptura exigua. vittata, Typocerus badlus, luguhris: Chrysomil. : Dienycha: Curculion, Centrinus pienunus

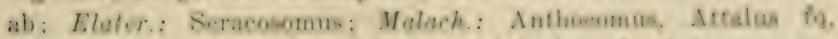
Melyris fị: Mordell.: Mowlellistena biplagiata bi. Intes, ornata, 
tosta, Pentaria fq; Nitidul.: Epurea labilis, truncatella; Oedermer.: Asclera puncticollis; Scarabae.: Trichius affinis, piger, Valgus.

Hemiptera (1)-Caps.: Lopidea.

On staminate and pistillate flowers (19) :-

Short-tongued Bees (10)-Halict.: Chloralictus sparsus $\delta^{\pi} q$,

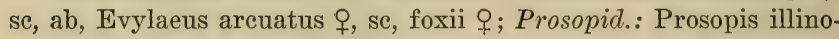
ensis $\sigma^{\lambda}+\mathrm{fq}$, saniculae $\delta^{\lambda}+\mathrm{fq}$, sayi $\delta^{\lambda}+$, ab, ziziae $q$, fq ; Andren. (q) : Andrena platyparia, Opandrena cressonii, Trachandrena crataegi, sc, ab.

Coleoptera (9)-Cerambyc.: Euderces ab, Leptura pubera; Dermest.: Anthrenus musaeorum ab, Cryptorhopalum haemorrhoidale fq, triste ab, Orphilus ab; Mordell.: Mordella marginata ab. Mordellistena aspersa fq, pubescens.

On pistillate flowers (16) :-

Long-tongued Bees (4)-Ceratin.: Ceratina + , Zaodontomerus 07 ; Nomad.: Gnathias cuneatus $q$, ovatus $q$.

Short-tongued Bees (7, \&)-Halict.: Agapostemon radiatus, Chloralictus tegularis, Curtisapis coriacea, Evylaeus pectoralis, Oxystoglossa confusa, pura; Andren.: Andrena pruni.

Other Hymenoptera (1)-Crabron.: Blepharipus.

Diptera (3)-Syrph.: Allograpta, Syrphus ribesii; Tachin.: Epigrimyia floridensis.

Coleoptera (1)-Cerambyc.: Molorchus.

The following 35 species and 70 individuals were taken on the flowers, June 8 and 11:

Long-tongued Bees (4:5)—Ceratin.: Ceratina 2, Zaodontomerus 1; Nomad.: Gnathias cuneatus 1, ovatus 1.

Short-tongued Bees (14:42)-Halict.: Agapostemon radiatus 1, Chloralictus sparsus 7, tegularis 1, Curtisapis coriacea 1, Evylaeus arcuatus 1, foxii 1, pectoralis 2, Oxystoglossa confusa 1, pura 1; Prosopid.: Prosopis illinoensis 3, saniculae 4, sayi 7, ziziae 2; Andren.: Andrena platyparia 1, pruni 1, Opandrena cressonii 1, Trachandrena crataegi 5.

Other Hymenoptera (1:1)-Crabron.: Blepharipus 1.

Diptera (3:3)-Syrph.: Allograpta 1, Syrphus ribesii 1; Tachin.: Epigrimyia floridensis 1.

Coleoptera (10:19)-Cerambyc.: Euderces 1, Leptura pubera 1, Molorchus 2; Dermest.: Anthrenus musaeorum 1, Cryptorhopalum haemorrhoidale 3 , triste 7 , Orphilus 1 ; Mordell.: Mordella marginata 1, Mordellistena aspersa 1, pubescens 1 .

Crataegus coccinea? (15:444-5).-Blooms April 13-May 22; 56 visitors observed, May 4-12; Mis, W. 
Long-tongued Bees (5) - Ap.: Apis se, fy: Bumb.: Bumbus americanorum o. fq, impations \&; Cerutin.: Crratina $\vec{\sigma}$; Nomol.: Centrias americanus $\delta$.

Short-tongued Bees (15)-Maliet. (f): (Chloralietus sparsus, versatus, zephyrus fq, Halietus lerouxii, Odontalictus, Seladonia

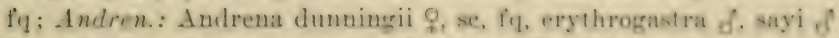
9. se, fq. Opandrena eressonii of ? miserabilis of Q, se, ah, persomata $\therefore$. fq. Trachandrena elaytoniate $\delta$. fq, rrataeri \& 9 ab, type, hippotes of.

Other Hymenoptera (3)-Vespl: Pulistes pallipes, fq. variatus; Tiphi.: 'Tiphia elypeata, type.

Diptera (2.)-Stratiomy.: Mirrochrysa; Syrph.: Eristalis aenens, dimiliatus, Helophilus similis, Malluta cimbieiformis, Mesugramma geminata. Myiolepta strigilata fq. P'ipiza nisribarba, Psilota, Syrphus torvus; Empil.: Empis avida type. Thamplomyia priapulus, sordida ; Bombyli.: Bombylius major, pulelellus ; Conop.: Mropa vesioulosa ; Tuhin.: Gonia eapitata; Sarcopha!g.: Calliphora erythrocephala, Cymomyia, Sareophaga sinuata; M/sc.: Compsomyia, Lurilia enesar, Morellia, Phormia regina, Psoudopyrellia fy.

Lepidoptera (2)-Nymphul.: Anosia, Vanessa huntera.

Coleoptera (6) - Corambuc.: Molorehus fq, in cop; Chrysomel.: Diabrotiea vittata; Cocein ll.: Coecinella sangninea, Hippodamea 15-malata: Oidemer.: Aselera puncticullis; Scarabat.: Euphoria fulgida.

Crataegus crus-galli $(15: 445-6)$.-Blooms May $1-J u m$ 1; 75 visitors observed, May 17-29; Mis, W.

Long-tougued Bees (9)-Ap.: Apis se, ab; Bumb. (q) : Bombins aurieonus, separatus, Bombus amerieanorum, impatiens; Coratin.: Ceratina of; Megachil.: Prochelostoma d. Anthemois d; Eucer.: Tetralonia dilecta $\widehat{\sigma}$.

Short-tongued Bees (26)-Malict. (f, se) : Chlorulietus pilious ah. sparsus ab, tegularis $\mathrm{fq}$, versatus ab, zephyrus. Evylaeus foxii fı. peetoralis, Oxystoglnssa similis fị, Selndonia ab; Prosmpil.: Prosupis erataegi $\delta$, illinoensis $\delta$, pygmaea $\delta 9$, ah, sanieulae $d$, ziziae o 8 , ab; Collet.: Colletes inaequalis 9. se; Andren.: Andrena dunningii 9. se, erythrogastra \%, mandibnlaris 9. se, sayi \%. sre Opandrena eresonii, of $\mathcal{F}$, se, fy, miserabilis of 9 . se. ah, imsumata of ?. se, ab. Parandrena 9. Trachandrena daytoniate o. se. fo, erataegi $\sigma^{\circ}$ ㅇ, sc, ab, forbesii 오, se.

Other Hymeunptera (10)-Vesp.: Polistes pallipes; Kumen.: Ancistroemsis eapra, tigris, Eumenes frateruus fq, Leionotus anormis. foraminatus, Symmorphus ibbilis, walshianus: Crobron.: Rhopalum seutellatum; Pemphredon.: Cemonus. 
Diptera (23)-Stratiomy.: Stratiomyia quaternaria; Syrph.: Didea, Eristalis dimidiatus, latifrons, transversus, Helophilus similis, Mallota cimbiciformis, Mesogramma geminata, Orthoneura nitida, Syritta, Volucella vesiculosa, Xanthogramma emarginata; Empid.: Empis distans, Rhamphomyia mutabilis; Bombyli.: Bombylius major; Sarcophag.: Cynomyia, Helicobia 4-setosa; Musc.: Morellia, Phormia regina, terraenovae; Anthomy.: Phorbia acra, platura; Ortal.: Camptoneura.

Lepidoptera (3)-Nymphal.: Anosia; Lycaen.: Cyaniris; Papilion.: Laertias.

Coleoptera (4)-Buprest.: Acmaeodera culta; Cerambyc.: Molorchus; Cler.: Enoplium; Scarabae.: Euphoria fulgida.

Crataegus mollis $(15: 443-4)$.-Blooms April 10-May 17; 73 visitors observed, April 27-May 9; Mis, W.

Long-tongued Bees (4)-Ap.: Apis se, ab; Bomb.: Bombus americanorum + ; Ceratin.: Ceratina $\delta \delta^{\top}$ Eucer.: Tetralonia dilecta $\widehat{\sigma}$ ㅇ.

Short-tongued Bees (28)-Halict. (q): Augochlora viridula, Chloralictus cressonii, pilosus sc, sparsus se, ab, versatus, zephyrus sc, ab, Evylaeus areuatus se, foxii sc, pectoralis se, fq, Halictus lerouxii sc, fq, Oxystoglossa confusa sc, Seladonia se, fq; Collet.: Colletes inaequalis $\delta^{\lambda}+$; Andren.: Andrena dunningii $q$, se, ab, mandibularis $q$, se, nasonii $\delta$, pruni $q$, se, sayi $\delta$, se, fq, Opandrena eressonii $\delta^{\top} q$, sc, ab, miserabilis $\delta^{\top} q$, fq, Trachandrena clay-

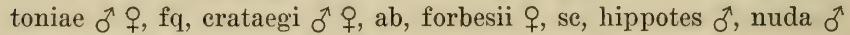
o, rugosa of, fq.

Other Hymenoptera (4)-Vesp.: Polistes pallipes, variatus, Vespula germanica; Eumen.: Ancistrocerus tigris.

Diptera (30)-Syrph.: Brachypalpus frontosus, Chilosia punctulata, Chrysogaster ontario, Criorhina umbratilis, Eristalis dimidiatus, Helophilus similis, Myiolepta strigilata fq, Orthoneura pietipennis, Pipiza nigribarba, Psilota, Syrphus americanus, Xylota fraudulosa; Empid.: Empis avida, humilis, nuda, otiosa, Rhamphomyia piligeronis, priapulus $f q$, sordida $f q$, in cop, tersa; Bombyli.: Bombylius major; Conop.: Myopa vesiculosa fq, in cop; Tachin.: Alophora grandis, cotype; Sarcophag.: Calliphora erythrocephala, vomitoria, Cynomyia; Musc.: Morella, Phormia regina, Pseudopyrellia; Anthomy.: Phorbia platura.

Lepidoptera (1)-Nymphal.: Polygonia interrogationis.

Coleoptera (6)-Cerambyc.: Molorchus; Chrysomel.: Diabrotica vittata, Disonycha; Cler.: Enoplium; Oedemer.: Asclera puncticollis; Scarabae.: Euphoria fulgida. 
Fragaria virginiana illinoensis $(15: 440)-$ Blooms April $1:$ June 11; 57 visitors observed, April 25-May 15; Mi, W.

Long-tongued Bees (12)-Ap.: Apis; Bomb.: Bombus im-

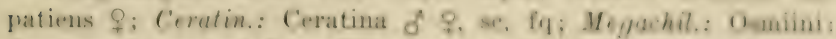
Osmia atriventris $\mathcal{E} \&$, se, illinoensis $\mathcal{f}$, pumila $\mathcal{Q}$, se: Comlioxyini: Coelioxys 8-dentata of Nomod.: Gnathias ovafus \&. Holonomaln superha of Nomala illinoensis \&; Melect.: Bombemelecta 9; Reen. 'Tetralonia dileeta of.

Short-tongued bees (15)-Mulivt. (9): Chloralietus pilosus se, fq, tegularis, versatus se, fq, Evylaens pertoralis se. fy, Odon talietus se, Oxystuglossa confusa se, pura se, similis se, ab, Seladonia se, ab: Sphecodini : Machaeris styeria; Prosopul. : Prosopis pramaea q. riziae \&: Andren.: (\&. se) : Indrena nasonii fy. Opamirena cressonii, 'Trachandrena forbesii.

Other Hymenoptera (1)-Vesp.: Polistes variatus.

Diptera (21) - Stratiomy.: Nemotelus: Syrph.: Eristulis trans. versus, Helophilus similis, Mrengramma marginata, Orthoneura pietipennis fig. Paragus bieolor fig. Spharrophoria fig. Tropulia mamillata; Empid.: Empis humilis, Thamphomyia friapulus: Bombyli.: Bombylius pulehellus; Conop.: Myopa vesienlesn. Zndion fulvifrons; Tuchin.: Gymnosoma, Peleteria; Surcophag.: Helimblia helicis, Sareophaga sinuata fy; Musc.: Lueilia caesar, sylyarum; Anthomy.: Phorbia acra, platura.

Lepidoptera (7)-Nymphal.: Phyeiodes; Lyearn.: Everes; Papiliom.: Eurynus, Pieris; Hesperi.: Pholisora catullus. Thanaos juvenalis; Noctu.: Plusia simplex.

Hemiptera (1)-Nab.: Coriseus.

Geum canadense $(15: 439)$,-Blooms June 15-Si.ptember 27; 22 visitors observed, July $6-11$; Mi, W.

Long-tongued Bees (1)-Pasit.: Ioleopasites illinw-usis ?

Short-tongued Bees (9)-Halict.: Chloralictus pilosns 8 . pruinosus \&, se, sparsus of, se, tegularis \&, versatus \&. so. Osystu.

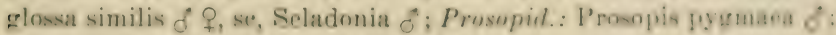
Panury.: Calliopsis andreniformis $\delta$.

Other Hymenoptera (6)-Philauth.: Cereris entuata: Eumen.: Eumenes fraternus, Leionotus histrionalis, siaiae; Cre bron.: Solenius; Chrysid.: Hedyehrum violaceum.

Diptera (3)-syrph.: Mesogramma marginata; Techin : ()eyp. tera dosiades; Sarcophag.: Helicobia helieis.

Coleuptera (2)-Cerambye. Euderees: Mondall. Mardella man ginata.

Hemiptern (1)-Corimelacn.: Corimelaena puliearia. 
Geum vernum $(15: 439-40)$.-Blooms April 12-May 24; the first 100 individuals, belonging to 18 species, were taken May 1-15; Mi, Y.

Long-tongued Bees (2:17)-Ceratin.: Zaodontomerus ơ, 1; Nomad.: Nomada parva ô. + , 16.

Short-tongued Bees (13:67)—Halict. (q): Chloralictus sparsus 7, tegularis 1, versatus 4, Evylaeus foxii 1, pectoralis 3, Oxystoglossa confusa se, 3, similis se, 6, Seladonia 3; Sphecodini: Sphecodium cressonii 1 ; Prosopid. $\left(\sigma^{\lambda}\right)$ : Prosopis pygmaea 2 , sayi 1 , ziziae 2 ; Andren.: Opandrena personata $\delta$ $\%, 33$.

Diptera (3:16)-Syrph.: Mesogramma geminata 2, Paragus bicolor 3; Tachin.: Siphona 11.

Gillenia stipulacea $(16: 169-70)$.-Blooms June 3-July 7; 25 visitors observed, June 15-30; Ma, W.

Long-tongued Bees (11)-Ceratin.: Ceratina + , se, ab, Zaodontomerus $\hat{\sigma}^{\lambda}$; Megachil.: Trypetini: Neotrypetes truncatus $\delta^{\lambda}+$, se, fq; Osmiini: Alcidamea simplex $\hat{\delta} q, \mathrm{sc}, \mathrm{ab}$, truncata $q$, se, Leucosmia $q$, se, Osmia atriventris $q$, distincta $q$, se, ab; Megachilini : Megachile addenda $q$, se; Nomad.: Holonomada affabilis $q$; Anthophor.: Anthemoessa of.

Short-tongued Bees (6)-Halict.: Chloralictus sparsus $q$, c, fq, $n$, versatus $q$, se, Evylaeus pectoralis $\delta$, se, 4 -maculatus $q$; Panurg.: Calliopsis andreniformis $\delta$, Heterosarus $\delta$, $q$, se, ab.

Diptera (4)-Syrph. (f, n) : Baccha fuscipennis, Pipiza nigribarba; Bombyli.: Bombylius atriceps; Conop.: Stylogaster biannulata $\mathrm{fq}$.

Lepidoptera (4)-Papilion.: Euphoeades, Laertias; Hesperi.: Thorybes bathyllus, pylades.

Potentilla canadensis (15:441).-Blooms April 23-June 17; 30 visitors observed, May 14-June 2; Mi, Y.

Long-tongued Bees (8)-Bomb.: Bombus americanorum $q$; Ceratin.: Ceratina $\sigma^{\lambda} \uparrow, \mathrm{fq}$, Zaodontomerus $\sigma^{\lambda}$; Stelid.: Microstelis $\widehat{\delta}$; Megachil.: Alcidamea simplex $\widehat{\delta}$, Osmia pumila + ; Nomad.: Centrias americanus $\hat{\delta}$, Nomada parva $\hat{\delta}$.

Short-tongued Bees (10)-Halict. () : Chloralictus versatus, Evylaeus pectoralis, Odontalictus, Oxystoglossa confusa se, ab, similis sc, ab, Seladonia; Sphecodini: Proteraner, Sphecodium cressonii; Prosopid.: Prosopis ziziae $\delta^{\dagger}$; Andren.: Opandrena personata $\hat{0}+$, ab.

Other Hymenoptera (4)-Eumen.: Leionotus anormis, clypeatus fq, histrionalis, ziziae. 
Diptera (7)-Syrph.: Paragus tibialis, Syritta: Tachin.:

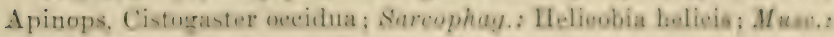
Lueilia eaesar; Anthomy.: Phorbia platura.

Lepidoptera (1)-Nymphal.: Phyeiodes.

Potentilla monspeliensis norvegica.-Blooms May 19 Xoven. ber $19 ; 23$ visitors observed, July 25,27 ; Mi, Y.

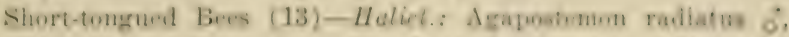

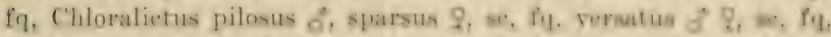
Evylacus pectoralis \&. Odontaliotus of. Oxgstogloma similis ?. to.

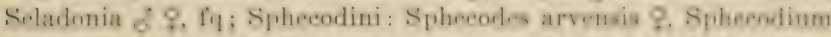

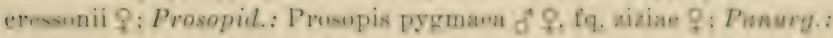
Calliopsis andreniformis $\delta$.

Other IIrmenoptera (1) - Orybel.: Notnglean frontalis fon.

Diptrra (8)-Syrph.: Mesogramua geminuta, uarginata. Sphavephoria fq. Syritta: Conop.: Oneusyia; Tsehin.: Gymmoprosopa; Sarcophag.: Helicobia helicis; Anthomy,: Phorlia sem.

Lepidoptera (1)-Lycacn.: Everes.

Prunus americana $(15: 4: 35-6),-$ Blooms Mareh 31-May $5 ; 76$ visitors observed, April 17-29; Mis, W.

Long-tongued Bees (14) - Ap.: Apis se. ab: Bomb.; Bombus americanorum of, impations of: Ceratin.: Coratina ob, ab, Zandontomerus d. iq: Nomml.: Ginathins ovatus of Heminnmala $\mathcal{Z} Q$, iq Holonomada superha $d$. Nomala dentirulata $\delta$. illinowensis of 8 . Phor of. Xanthidium luteoloides 8, luteolum ?: Eucr.: Tomblenis dilecta $\sigma^{+}$o, ab.

Shart-tongued Bees (26)-Holiet (Q): Agapontemon radiatus, Chloralietus pilosus sc, ab, jruinosus se, ab, sparsus, tocularis, ver. satus so, ab, zephyrus se. Fvylaeus arenatus, 1falietus lerousii $=$. Seladonia se; Sphrendini: Spheeodes arvensis; Prosopil.: Prompin

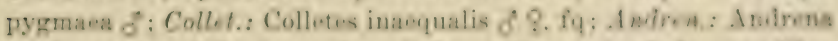
erythrogastra of . ab, erythronii 8 , se, illinoensis $d$, Pr, mandihe

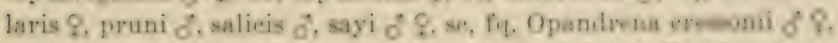
sa, ab, mis rabilis $\delta$ Q $\mathrm{se}$, ab, Parandrena $\delta$, ab. Trachindrens claytoniae $\delta$ o $q, \mathrm{se}, \mathrm{ab}$, forbesii $\delta$, hippotes of $q, \mathrm{se}, \mathrm{fq}$.

Other Ilymenopiera (3)-Vup. Polistes palliges, saviatua: Ichnoumon.: Bathycetes.

Diptera (23) - Syrph.: Brachypelpus froniosus, Ciryoucater onturio, Eriatalis dimiliatus, Helephilus nimilis. Mesosmetoms geminata, marginata ab. Orthoneura nitias, Platycbiros hyser. boreus. Spharerophoria, Syrphos amerisanes, tervas: Remple: Empis otiose fq, Rhampihemyin prinyulas; Rembylh: Bombylles 
major; Conop.: Myopa vesiculosa; Tachin.: Euphorocera claripennis, Gonia capitata fq; Sarcophag.: Cynomyia fq; Musc.: Phormia regina, Pseudopyrellia; Anthomy.: Anthomyiella, Phorbia platura ab; Oscin.: Chlorops versicolor.

Lepidoptera (7)-Nymphal.: Anosia, Vanessa atalanta, huntera; Papilion.: Jasoniades, Pontia fq; Hesperi.: Thanaos juvenalis; Noctu.: Plusia simplex.

Coleoptera (3)-Cerambyc.: Molorchus; Chrysomel.: Orsodachna; Scarabae.: Valgus.

Prunus serotina (15:436-7).-Blooms April 9-May 23; 64 visitors observed, April 9-May 18; Mis, W.

Long-tongued Bees (7)-Ap.: Apis se, ab; Bomb. (q) : Bombias separatus, Bombus americanorum fq, impatiens; Ceratin.: Zaodontomerus $\delta^{\lambda}$; Nomad.: Nomada illinoensis $q$; Eucer.: TetraIonia dilecta $\delta$.

Short-tongued Bees (23)-Halict.: Agapostemon radiatus ô, fq, Augochlora viridula $q$, Chloralictus cressonii + , se, pilosus $q$, sc, sparsus + , se, fq, versatus $q$, se, fq, zephyrus + , sc, Curtisapis coriacea $q$, sc, fq, Halictus lerouxii $q$, sc, fq, Oxystoglossa similis $q$, sc, fq, Seladonia $q$, sc; Collet.: Colletes inaequalis $q$; Andren.: Andrena erythrogastra $q$, pruni $q$, sc, fq, type, sayi $q$, sc, Opandrena cressonii $\delta q$, se fq, miserabilis $q$, se, ab, Trachandrena claytoniae $q$, sc, fq, crataegi $\delta^{\lambda}$, forbesii $q$, se, hippotes $\delta^{\lambda}$, nuda + , se, rugosa $\sigma^{2}$.

Other Hymenoptera (2)-Vesp.: Polistes pallipes; Tenthredin.: Dolerus sericeus.

Diptera (29)-Bibion.: Seatopse notata fq; Simuli.: Simulium; Stratiomy.: Stratiomyia quaternaria; Syrph.: Brachyopa, Eristalis aeneus fq, dimidiatus, Mallota cimbiciformis, Myiolepta strigilata, Orthoneura nitida, pictipennis, Pipiza nigribarba, Sphaerophoria, Sphegina rufiventris, Syritta, Syrphus ribesii; Empid.: Rhamphomyia sordida; Conop.: Myopa vesiculosa; Tachin.: Gonia capitata, Siphona; Sarcophag.: Calliphora erythrocephala, Cynomyia, Sareophaga cimbicis; Musc.: Lucilia caesar, sericata, Phormia regina, Pseudopyrellia; Anthomy.: Phorbia acra, platura; Scatophag.: Seatophaga.

Lepidoptera (2)-Nymphal.: Anosia, Vanessa huntera.

Coleoptera (1)-Cerambyc.: Molorehus.

Extra-floral nectaries.-April 25, 1894, the following insects were observed sucking:

Short-tongued Bees (6)-Halict. ( $q)$ : Chloralictus illinoensis, sparsus, versatus; Andren.: Andrena erythrogastra $q$, illinoensis $\sigma^{\lambda}$, Opandrena miserabilis ㅇ․ 
Other Hymenoptera $(2, \not ̧)-F o r m i c .:$ Formica fusca; Myrmic.: Cremastogaster.

Diptera (2)—Syrph.: Criorhina umbratilis; Sarcophag.: Cynomyia.

Pyrus coronaria (15:442-3).-Blooms April 10-May 23; 9 visitors observed, May 2-12; Ma, R.

Long-tongued Bees (7)-Ap.: Apis fq;Bomb. (q) : Bombias auricomus, separatus fq, Bombus americanorum ab, impatiens ab; Megachil.: Ceratosmia + ; Eucer.: Tetralonia dilecta $\delta$, fq.

Lepidoptera (2)-Nymphal.: Anosia; Hesperi.: Thanaos juvenalis.

Rosa humilis (15:441-2).-Blooms May 15-July 29 ; 27 visitors observed, May 22-June 20 ; Ma, R.

Long-tongued Bees (8, c)-Bomb.: Bombias separatus $q \not$, Bombus americanorum $q$, ab, impatiens $q$; Ceratin.: Ceratina $q$; Megachil.: Megachile brevis $q$; Eucer.: Tetralonia dilecta $q$, ab; Anthophor.: Anthemoessa $q$, ab, Clisodon $q$.

Short-tongued Bees (6, $q$, c)-Halict.: Agapostemon radiatus, viridulus, Augochlora fervida, Chloralictus versatus, Oxystoglossa confusa ; Andren.: Opandrena cressonii.

Diptera (11, f)-Syrph.: Allograpta n, Mallota cimbiciformis, posticata, Mesogramma geminata $n$, Baccha fuscipennis $n$, Rhingia $\mathrm{n}$, Syrphus americanus n, Volucella vesiculosa fq, n, Xylota chalybea $\mathrm{n}$, fraudulosa $\mathrm{n}$; Bombyli.: Anthrax sinuosa.

Coleoptera (2, f)-Chrysomel.: Diabrotica 12-punctata n; Scarabae.: Trichius piger ab.

Rosa setigera (15:442).-Blooms June 9-July 21; 35 visitors observed, June 14-July 7 ; Ma, R.

Long-tongued Bees (12, c)-Ap.: Apis fq; Bomb.: Bombias auricomus $q \not$, separatus $q \not{\gamma}, \mathrm{ab}$, Bombus americanorum $q \not, \mathrm{fq}$, impatiens $\not$; Megachil. ( $q$ ): Osmia pumila, Megachile addenda, mendica fq, Xanthosarus; Eucer.: Tetralonia dilecta $q$, fq, rosae q, type, Anthophor.: Anthemoessa $q$, ab.

Short-tongued Bees (6, $q, \mathrm{c})$-Halict.: Agapostemon radiatus, viridulus fq, Augochlora viridula, Chloralictus versatus, Curtisapis coriacea fq, Evylaeus arcuatus.

Diptera (16, f)-Syrph.: Allograpta n, Eristalis transversus, Mallota cimbiciformis fq, posticata, Milesia fq, Myiolepta nigra $n$, Pipiza pistica n, pulchella n, Syritta n, Syrphus americanus fq, n, Temnostoma $\mathrm{n}$, Xylota angustiventris $\mathrm{n}$, chalybea $\mathrm{n}$; Bombyli.: Anthrax sinuosa, Argyramoeba albofasciata, oedipus.

Coleoptera $(1, \mathrm{f})$-Scarabae.: Trichius piger. 
Rubus canadensis.-Blooms May 2-June 29; 48 visitors observed, May 10-26; Mi, W.

Long-tongued Bees (16)-Ap.: Apis fq; Bomb.: Bombus americanorum + , ab; Ceratin.: Ceratina o $o^{\lambda}$; Megachil.: Osmiini: Alcidamea simplex $\delta^{\lambda}+$, se, Ceratosmia $q$, se, Hoplitis $\widehat{\jmath}+$, se, Osmia atriventris $q$, se, cordata $q$, pumila $q$, se, fq; Megachilini : Megachile brevis $\sigma^{\lambda}$, mendica $\sigma^{\lambda}$; Coelioxyini : Coelioxys sayi $\sigma^{\lambda} q$; Nomad.: Centrias americanus $q$, Holonomada superba $o^{\lambda}+$, fq; Eucer.: Tetralonia dilecta $\delta$ $q$, fq, rosae $\delta$ ㅇ․

Short-tongued Bees (15)-Halict. ( $q)$ : Agapostemon viridulus sc, Chloralictus versatus se, Evylaeus pectoralis sc, Odontalictus, Oxystoglossa similis se; Andren.: Andrena carlini $q$, nasonii $q$, se, pruni $q$, se, Opandrena cressonii $q$, se, personata $\delta$, Trachandrena claytoniae $q$, se, crataegi $q$, se, forbesii $q$, hippotes $q$, se, nuda o se.

Other Hymenoptera (2)-Eumen.: Leionotus foraminatus, scrophulariae.

Diptera (6)—Syrph.: Eristalis dimidiatus, latifrons, Tropidia quadrata; Bombyli.: Bombylius atriceps fq; Conop.: Zodion fulvifrons; Sarcophag.: Helicobia helicis.

Lepidoptera (9)-Nymphal.: Charidryas; Papilion.: Eurymus; Hesperi.: Atrytone zabulon, Epargyreus, Limochores taumas, Pholisora catullus, hayhurstii, Thanaos juvenalis, Thorybes pylades.

Rubus occidentalis $(15: 437-8)$.-Blooms May 3-June 9; 22 visitors observed, May 12-29; Mi, W.

Long-tongued Bees (10)-Ap.: Apis fq; Bomb.: Bombias separatus $q$, Bombus americanorum $q ;$ Megachil.: Alcidamea simplex $\delta$, Osmia pumila + , Megachile brevis $q$; Nomad.: Gnathias ovatus $q$, Holonomada affabilis $\delta$, fq, superba $\delta^{\tau}+$, fq ; Eucer.: Tetralonia dilecta $\sigma \times$ ㅇ.

Short-tongued Bees $(5$, 우)-Halict.: Odontalictus, Oxystoglossa confusa se, Seladonia; Andren.: Andrena carlini se, Opandrena cressonii.

Other Hymenoptera (2)-Eumen.: Leionotus anormis; Crabron.: Solenius.

Diptera (1)-Tachin.: Archytas aterrima.

Lepidoptera (4)-Nymphal.: Charidryas; Hesperi.: Limochores taumas, Pholisora catullus, Polites.

Rubus villosus (15 : 438-9).-Blooms May 2-July 1; 89 visitors observed, May 12-June 16; Mi, W.

Long-tongued Bees (23)-Ap.: Apis sc, ab; Bomb. (q): Bombias auricomus, separatus se, Bombus americanorum se, fq, im- 
patiens, Psithyrus variabilis; Ceratin.: Ceratina $\delta$, se, $f q$; Megachil.: Osmiini : Alcidamea simplex $\sigma^{\wedge} \uparrow, \mathrm{se}$, Ceratosmia $q, \mathrm{se}, \mathrm{fq}$, Hoplitis $q$, se, Osmia pumila $q$, se; Megachilini: Anthemois $\delta^{7}$, Megachile brevis $\delta$, mendica ' $q$; Coelioxyini: Coelioxys 8-dentata $\sigma^{\lambda}$, sayi $\delta^{\lambda}$; Nomad.: Gnathias ovatus $q$, Holonomada affabilis $\delta^{\lambda} q$, fq, superba $\delta^{\lambda}+$, fq, Nomada sayi $q$; Eucer.: Tetralonia dilecta $\delta$, $q$, in cop, rosae $\delta$; Anthophor.: Anthemoessa $q$.

Short-tongued Bees (27)-Halict. ( $(9)$ : Agapostemon radiatus, Augochlora fervida se, viridula se, Chloralictus sparsus c, n, versatus e, n, Curtisapis forbesii se, Evylaeus pectoralis se, fq, Halictus lerouxii, Odontalictus se, Oxystoglossa confusa sc, similis sc, Paralictus simplex, Seladonia sc, fq; Sphecodini: Sphecodes arvensis, Sphecodium cressonii; Prosopid.: Prosopis pygmaea $q$; Collet.: Colletes aestivalis 0 , inaequalis $q$, sc; Andren. ( $q)$ : Andrena carlini, dunningii, mandibularis, sayi se, Opandrena personata se, Trachandrena claytoniae, crataegi se, forbesii se, nuda.

Other Hymenoptera (7)-Sphec.: Isodontia apicalis, Sphex nigricans; Eumen.: Leionotus arvensis, histrionalis, vagus, ziziae; Crabron.: Solenius.

Diptera (16)—Syrph.: Didea f, n, Eristalis dimidiatus f, n, Mesogramma marginata $f, n$, Paragus bicolor n, Sphaerophoria, Syritta f, Syrphus ribesii f, n, Tropidia mamillata, Volucella vesiculosa fq; Empid.: Empis distans; Bombyli.: Aldrichia f, fq, n, Bombylius atriceps; Conop.: Zodion fulvifrons; Tachin.: Archytas aterrima, Ocyptera tenuis, Spallanzania.

Lepidoptera (13)-Nymphal.: Anosia, Charidryas, Vanessa atalanta; Lycaen.: Chrysophanus; Papilion.: Eurymus, Pontia; Hesperi.: Atrytone zabulon, Epargyreus, Euphyes metacomet, Limochores taumas, Polites $\mathrm{f} q$, Thanaos juvenalis; Ctenuch.: Scepsis.

Coleoptera (3)-Scarabae.: Euphorea fulgida f, n, sepulchralis $f, n$, Trichius piger sf.

\section{RUBIACEAE}

Cephalanthus occidentalis $(4: 65-7)$.-Blooms June 27-August 25; 77 visitors observed, July 5-August 17; Mas, W.

Long-tongued Bees (25)-Ap.: Apis ab; Bomb.: Bombias auricomus $q \not \succ, f q$, scutellaris $\not$, separatus $\delta q \not \phi, a b$, Bombus ameri-

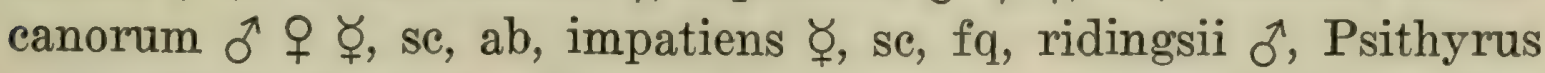
variabilis $q$; Ceratin.: Ceratina $q$; Megachil.: Megachile brevis $\delta^{\lambda} q$, fq, mendica $q$, c, petulans $q, 6$-dentata $o^{\top} q$, Sayapis sayi $q$; Nomad.: Cephen $q ;$ Epeol. ( $\delta^{\top}$ ㅇ) : Triepeolus coneavus, concolor fq, lunatus; Eucer.: Epimelissodes obliqua $\delta^{\lambda}+$, sc, fq, Florilegus + , Melissodes 
bimaculata $\delta$, petalostemonis $q$, variabilis $q$, se, Peponapis $\delta$; Emphor.: Emphor ㅇ.

Short-tongued Bees (6)-Halict.: Agapostemon radiatus ô, texanus $q$, viridulus $q$, Halictus lerouxii $q$, Odontalictus $q$; Prosopid.: Prosopis ziziae $q, f, \mathrm{n}$.

Other Hymenoptera (4)—Sphec.: Sphex nigricans; Scoli.: Elis 5-cincta; Eumen.: Leionotus arvensis; Pompil.: Prioenemoides fulvicornis.

Diptera (10)-Syrph.: Eristalis latifrons, tenax ab, Sphaerophoria, Syritta, Volucella evecta, vesiculosa; Conop.: Conops brachyrrhynchus, Physocephala texana, tibialis; Musc.: Musca.

Lepidoptera (29)-Nymphal.: Anosia ab, Argynnis, Basilarchia archippus, Cercyonis, Phyciodes ab, Vanessa atalanta ab, cardui, huntera; Lycaen.: Chrysophanus ab, Cyaniris, Everes, Uranotes; Papilion.: Euphoeades, Eurymus, Laertias, Papilio ab, Pieris, Pontia, Zerene; Hesperi.: Atalopedes, Atrytone delaware, zabulon, Epargyreus ab, Limochores taumas ab, Polites, Thanaos juvenalis, Thorybes bathyllus; Arcti.: Utetheisa; Ctenuch.: Scepsis.

Coleoptera (f, n)-Coccinell.: Hippodamea 15-maculata; Scarabae.: Trichius piger.

Hemiptera (1)-Lygae.: Oncopeltus.

July 9, 1913, the following 74 individuals, of 21 species, were taken on the flowers :

Long-tongued Bees (10:56)-Ap.: Apis 32; Megachil.: Megachile brevis 5, petulans 2, 6-dentata 1, Sapapis sayi 1; Epeol.: Triepeolus coneavus 3, concolor 5, lunatus 2; Eucer.: Epimelissodes obliqua 2, Melissodes bimaculata 3 .

Short-tongued Bees $(1: 1)$-Halict.: Halictus lerouxii 1.

Other Hymenoptera (3:4)-Sphec.: Sphex nigricans 2; Scoli.: Elis 5-cincta 1; Eumen.: Leionotus arvensis 1.

Diptera (1:1)-Conop.: Physocephala texana 1.

Lepidoptera (6:12)-Nymphal.: Argynnis 1, Phyciodes 1; Lycaen.: Chrysophanus 1; Papilion.: Eurymus 2, Pontia 6; Hesperi.: Thorybes bathyllus 1 .

Galium aperine.-Blooms May 4-June 22; 1 visitor observed, May $15 ; \mathrm{Mi}, \mathrm{W}$.

Short-tongued Bees (1)-Halict.: Chloralictus obscurus q.

Galium trifidum.-Blooms May 22-July $9 ; 8$ visitors observed, June 26 ; Mi, W.

Short-tongued Bees (1)-Prosopid.: Prosopis pygmaea $\sigma^{7}$.

Diptera (7)—Syrph.: Mesogramma marginata, Pipiza pubescens, Syritta; Tachin.: Cistogaster occidua, Euphorocera claripennis; Sarcophag.: Helicobia helicis; Anthomy.: Coenosia antica. 
Houstonia purpurea calycosa (5: 275).-Bloums May 18-July 4; 37 visitors observed, May 25-June 12; Mi, W.

Long-tongued Bees (9) - Ip.: Apis; Bimbe: Rombias separatus $q$; Cerulin.: Ceratina of $\mathcal{f}$, se, ab; Mrguchil.: Neotrypetes productus of, type, Aleidamea simplex of; Nomad. (8): Centrias amerieanus, Holonomada affabilis, superba; Eucre.: Tetralonia rosae $\delta$.

Short-tongued Bers $(9)-M a l i r t .(9)$ : Chloralietus albipennis se, pilosus se, versatus se. Evylaeus pectoralis, Odontalietus, Oxysto. glusia confusa se, similis se. Seladonia ; Panurg.: Calliopsis andreni. formis $\delta \circ$, sc, $a b$.

Diptera (9) - Syrph.: Eristalis dimidiatus, Mesugramua uargi. nata, Paragus bieolor, tibialis, Sphasrophoria se, ab, Syritta; Bombyli.: Bombylins atriceps; Tochin.: Arrhytas analis, Spallanzania.

Lepidoptera $(T)-N y m p h a l$ : Charidryas fq: Lycuen.: Chrysos. phanus $\mathrm{f}_{\mathrm{q}}$; Papilion.: Pirris, Pontia; Hesperi.: Aneylexypha ab. Pholisora eatullus, Polites.

Coleoptera (3)-Curculion. (i. n) : Centrinites, Centrimus sm. tellum-album; Scarabae.: Trichius piger sf, ab.

\section{Rutaceas:}

Ptelea trifoliata $(9: 15,-7)$ - Blooms May $7-0$ une 23; tis vis. itors observed, May 22-June 8 ; Mis, W.

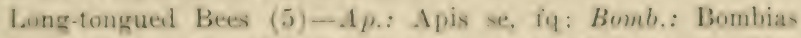
auricomus 8. se: Ceratin.: Ceratina 8. se. ab: Nomed.: Ginathias ovatus $q$; Eucer.: Tetralonia dilecta + , se.

Short-tongued Bees (2s)-IIalict. (9) : Agapostemon radiatus. viridulus, Chloralietus eressonii se, pilosns, sparsus se, tegularis so. versatus se, Curtisapis eoriacea, Halietus lerouxii, Odontalirtus. Uxystoglonsa ronfusa: Spleeodini: Drepanium fq : Prosopid: Promopis sayi $\delta$, ziziat. $\delta$ : Collet.: Colletes eulophi $\delta$, inarugualis 8. se: Andren.: Aulrena platyparia of $\mathrm{f}$. se, Opandwna eresonii ?. miserabilis $q$ se, $\mathrm{fq}$, serotina $q$, se, $\mathrm{fq}$, Trachandrena claytoniac $q$, crataegi $\mathcal{q}, \mathrm{sc}$, forbesii $q$, heraclei $\mathcal{q}, \mathrm{sc}$, hippotes $ᄋ$ se, ab, type, nuda $q$, se, fq, rugosa $q$, spiraeana ${ }^{\circ}$.

other IIymenoptera $(14) \rightarrow$ Sphec: Splex rulgaris; Scoli: Campsomeris; Philanth: Cereeris compar: Eumen.: Aneistraerus tigris, unifusciatus, Eumenes frateruus, L-ionotus elypeatus, foraminatus, scrophularias, ziziae; Crobron. Solenins; Orybel: Notoglossa amerieana, inornata; Chalcil.: Leueospis.

Diptera 19)-struliomy. stratomyia meimaic; syrph: Mat. lus eimbiciformis, Mryoleme nigm, Sphaerophiarin, Syriba, Volu.

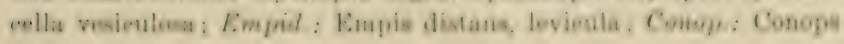


brachyrrhynchus, Dalmannia, Myopa vesiculosa ; Tachin.: Archytas analis, aterrima, Linnaemyia, Trichopoda pennipes; Sarcophag.: Helicobia helicis; Musc.: Pseudopyrellia; Anthomy.: Anthomyiella, Phorbia platura fq.

Lepidoptera (2)-Nymphal.: Neonympha; Agarist.: Alypia.

May 27, 1911, the following 22 species and 44 individuals were taken on the flowers:

Long-tongued Bees $(4: 21)-A p .:$ Apis $6 ; B o m b .:$ Bombias auricomus 1 ; Ceratin.: Ceratina 13 ; Eucer.: Tetralonia dilecta 1.

Short-tongued Bees (11: 16) - Halict.: Agapostemon viridulus 1, Chloralictus sparsus 3, tegularis 1; Prosopid.: Prosopis ziziae 1; Collet.: Colletes eulophi 1; Andren.: Opandrena cressonii 1, miserabilis 2, serotina 1, Trachandrena crataegi 2, heraclei 2, hippotes 1.

Other Hymenoptera (4:4)-Sphec.: Sphex vulgaris 1; Philanth.: Cerceris compar 1; Crabron.: Solenius 1; Oxybel.: Notoglossa americana 1 .

Diptera (3: 3)-Syrph.: Sphaerophoria 1, Syritta 1; Empid.: Empis levicula 1.

Zanthoxylum americanum (7:109-11).-Blooms March 31April 28; 59 visitors observed, April 9-25; Mis, Y.

On staminate flowers (7):--

Short-tongued Bees $(2$, १)-Halict.: Chloralictus cressonii, Curtisapis coriacea.

Other Hymenoptera (3)-Vesp.: Polistes pallipes; Pompil.: Cryptocheilus conicus; Chrysid.: Holochrysis hilaris.

Diptera (1)—Syrph.: Brachypalpus frontosus.

Coleoptera (1)-Oedemer.: Asclera puncticollis.

On staminate and pistillate flowers (16):-

Long-tongued Bees (4, ab)-Ap.: Apis se; Megachil.: Osmia pumila $\sigma^{\star}$; Nomad.: Gnathias cuneatus $0^{\wedge}$ o, type, Xanthidium luteolum $0^{\lambda}$ ㅇ.

Short-tongued Bees (10)-Halict. (o) : Chloralictus sparsus sc, $a b$, versatus sc, ab, Oxystoglossa confusa; Collet.: Colletes inaequalis $\hat{\sigma} q, a b$, in cop; Andren.: Andrena sayi $\hat{\delta}$, ab, Opandrena cressonii $\delta \tau, a b$, miserabilis $\delta q, a b$, Trachandrena claytoniae $\delta \tau$, $\mathrm{ab}$, forbesii $\sigma^{\lambda}$ \% , fq, rugosa $\delta^{\lambda}$, fq.

Diptera (2)-Syrph.: Mesogramma geminata ab; Tachin.: Gonia capitata ab.

On pistillate flowers (36):-

Long-tongued Bees (8)-Ceratin.: Zaodontomerus $\sigma^{\lambda}$; Megachil.: Ceratosmia $\delta^{\lambda}$ ㅇ, ab; Nomad.: Gnathias ovatus $\delta^{\lambda}$, fq, Nomada cres- 


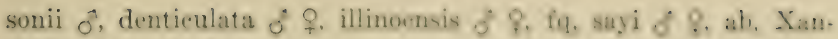
thidium luteoloides 0 .

Short-tongued Bees (12)-IIalict. (8): Augiolhlare viridula, Chloralietus caeruleus iq, zphyrus fq, Evylaeus areastus, foxii fq. Ilalietus lerouxii, Odontalietus, Paralietus platyparins; Andren. Andrena mandibularis of pruni $\delta$ \&. iq, Opaudrua dubia $\delta$. 14 . 'T'rachandrena hippotes $\delta$.

Diptera (15) - Syrph.: Eristalis dimidiatus, Heloghilus similis. Orthoneura nitida, Sphacrophoria, Syrphus americanus, Xaurhogranma Celix; Conop.: Myopa vesieulna; Tachin.: Arehyias analie. Gonia exul ab, Phorichacta, Phyto: Surcophay.: Helieobia 4 netian; Muse.: Phormia regina, Pscudopyrellin fiq; Scatophag, : Scatophage.

Lepidoptera (1)-Noctu.: Plusia simplex.

\section{SAlicicede}

Populus deltoides (anemophilous).-One visitor observed, April 14.

Long-tongued Bees (1) - A p.: Apis c, ab.

Populus tremuloides (anemophilous),-One visitor observed, April 6.

Long-tongued Bees (1)-Ap.: A pis c, ab.

Salix amygdaloides.-Blooms April 4-May 9; 97 visitors observed, April 10-28; Mis, Y.

On staminate flowers:

Long-tongued Bees (4)-Cerutin.: Ceratina of, Zaodoutomerus $\delta$; Nomad. Heminomada $q$, Nomada sayi $\delta$.

Short-tongued Bees (13)-Ilalict. (\$): Chloralietus illinomsis. Evylaeus arenatus se, fq, truneatus se, Oxystogloma coufusa se; Spheeodini: Spheendium eressnnii; Collet.: Colletes innequalis of

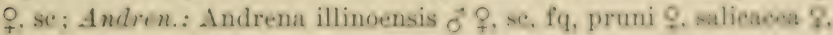
se, salietaria ${ }^{\circ}$ se, Opandrena personata of. Trachandrena erabargi oิ, nuda $q$, se.

Other Hymenoptera (2)--Visp.: Vespula grrmanics; Pompdi.: Cryptocheilus conicus.

Diptera (b) -Syrph.: Chryongaster ontario, Helophilus lati. frons. Psilota, Tropidia uauillata, Sylota fraudulos: Einmd.: Rhamphomyia irregularis: Bombyli.: Bombylius major: Mus: Morellia.

On staminate and pistillate flowers:

Long-tongued Pose $(7)-A p$ : Apis se, ab; Nomad: Cinabias

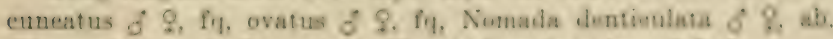
illinowsis of 9 , ab, Phor $\delta$, ab. Xanthidium lucolem of \&, fo. 
Short-tongued Bees (23)-Halict. (q) : Agapostemon radiatus, Chloralictus pilosus sc, fq, sparsus sc, ab, versatus sc, ab, zephyrus sc, ab, Evylaeus foxii sc, ab, pectoralis se, fq, Halictus lerouxii se, ab, Odontalictus, Oxystoglossa pura, Seladonia sc, fq; Andren. ( $\delta^{7}$ ㅇ) : Andrena dunningii, erythrogastra sc, ab, mandibularis sc, salicis se, sayi fq, Opandrena cressonii se, ab, in cop, miserabilis se, ab, Parandrena se, ab, Trachandrena claytoniae se, fq, forbesii sc, fq, hippotes sc, fq, mariae sc, fq.

Diptera (7)—Syrph.: Brachypalpus frontosus, Chilosia punctulata, Sphaerophoria; Empid: : Rhamphomyia limbata fq, priapulus fq ; Conop.: Myopa vesiculosa ab; Musc.: Pseudopyrellia.

On pistillate flowers:

Long-tongued Bees (3)-Bomb.: Bombus americanorum $q$; Megachil.: Osmia pumila $\delta^{\lambda}$; Nomad.: Nomada salicis $\sigma^{\lambda}$, type.

Short-tongued Bees (6)-Halict. ( $($ ) : Chloralictus eressonii, tegularis fq, Curtisapis forbesii; Andren. ( $\left.\sigma^{\top}\right)$ : Andrena carlini, erythronii, Trachandrena rugosa.

Other Hymenoptera (5)-Vesp.: Vespula germanica; Pompil.: Cryptocheilus conicus; Ichneumon.: Glypta rufiscutellaris fq ; Tenthredin.: Dolerus arvensis, Macrophya epinota, pleuricincta, Macroxyela.

Diptera (18)-Syrph.: Eristalis dimidiatus, Melanostoma obscurum, Mesogramma marginata, Platychirus quadratus, Syrphus americanus, torvus; Empid.: Rhamphomyia gilvipilosa; Conop.: Myopa pilosa ab, in cop; Tachin.: Gonia capitata, exul; Sarcophag: Cynomyia; Anthomy.: Coenosia antennalis, Hyetodosia, Mydaea fusca, Phorbia acra, platura fq; Scatophag.: Seatophaga; Seps.: Sepsis.

Coleoptera (1)-Chrysomel.: Orsodachna.

Salix cordata (9:76-7).-Blooms March 18-April 30; 123 visitors observed, March 25-April 29; Mis, Y.

On staminate flowers:

Long-tongued Bees (6)-Megachil.: Osmia pumila $\widehat{\sigma}+$, ab; Nomad.: Nomada denticulata $\delta^{\lambda}$, type, illinoensis $\sigma^{\top}+$, fq, type, sayi $\delta$, Xanthidium luteoloides $\delta$, luteolum or $q, \mathrm{ab}$.

Short-tongued Bees (16)-Halict. (q): Augochlora viridula, Chloralictus illinoensis, tegularis, Odontalictus, Oxystoglossa confusa $\mathrm{fq}$, similis type; Andren.: Andrena dunningii $\delta$, erythro-

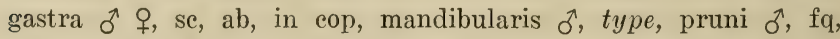

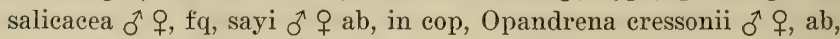
dubia $\delta$, type, Trachandrena hippotes $\delta$, fq, nuda $q$. 
Other Ilymenoptera 15 ) - Visp.: Polistes ruhiginesus; Pronpal: Cryptocheilus emieus: Tenthedin.: Dolerus bicolor. Nematus luteotergum, vertebratus.

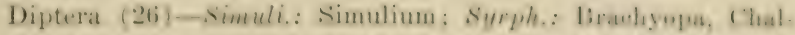
comyia, Chrysogaster ontario, Eristalis aeneus ab, dimidatue ab.

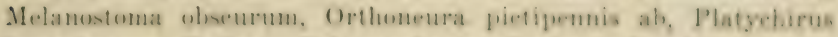
lyperboreus, Psilota, Suharrophoria, Syrita. Syrphus tarvos. Tropidia mamillata, Xylota fraudulosa: Empil. Empis otoona. Rhamplomyia priapulus: ('omop.: Myoja vesirulnan; Tachin: Gonia exul; Sorcophag.: Helieobia 4-setesal: Anthemen: Hyete dosia; Lonchoe.: Lonchaea aberrans, parvicornis; Osin.: Elachi. ptera eostata; Sciomys.: Siomyna, Tetanoera; Seps.: Proeliyliza

Coleoptera (3)-('hrysumel.: Galerura fy, Lina ab; lledemer : Asclera puncticollis fq.

On staminate and pistillate flowers:

Long-tongued Bees (7) -...Ap.: Apis se, ab: Ceralin.: Crratiua d. fq: Megachil.: Ceratosmia of ab: Nomad. $(8 \%)$ : Gnathias pune atus ab, ovatus ab, type. Nomada simplex fy. type. Phor integer ab, type.

Short-tongued Bees $(23)-$ Malict.: Arapostemon radiatus ?. Chloralietus pilosus o. fq. sparsus f, se, ab, versatus \&, fq. zephyrus f. ab, Curtisapis forbesii f, so, Evylateus arruatus \&, foxii \&. se, ah. type. Halictus lerouxii \&. fy. Paralietus platyparius \& Q. S.eladonia Q. fq: Spheeodini : Spheeodes arvensis 8. fq; Collet.: Colletes in.

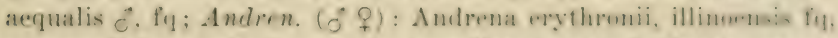
type, salieis se. ab, in cop, type, salietaria se, ab, in cope, type. Opandrena miserabilis ab, in cop. Parandrena ab, in cop, Trachand. rena claytoniae ab, forbesii $\mathrm{f}(\mathrm{s}$, mariac se, ab, in cop, t!ppe, rugosa $\mathrm{fq}$.

Other Hymenoptera (1)-Tenthratin.: Dolerus arvensis ab.

Diptera (14)-Syrph.: lirachypalpus frontosus ah, rileyi ab, Eristalis flavipes, Helnphilus similis, Platyohirus quadratus ab, Syrphus americamus ab, Xylota fraululona ab; Iinpid. Tham. phomyia gilvipilosa fq. type: Comop.: Myopa pilusa fy: Tanhon.: Conia capitata ab: Sarcophey.: Cynomyia ab: Mans: Proudon pyrellia ab: Anthomy.: Thorbin platura fo: Solopheg: Seam. phaga ab.

Coleoptera (1)-C'hrysomel.: Orsodachna ab.

On pistillute flowers:

Long-tongued Bees (1)-Ceratin.: Zaodontomerus ơ, fy.

Short-tongued Bees $(6, q)$-IIalict.: Chloralictus cressinnii,

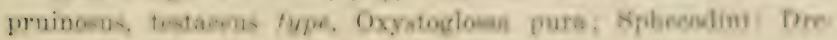
panium fq; Andron.: Andrena earlini. 
Other Hymenoptera (1)-Vesp.: Polistes variatus.

Diptera (11)—Syrph.: Eristalis transversus, Merapioidus; Tachin.: Euphorocera cinerea, claripennis, Panzeria, Tachina robusta; Sarcophag.: Calliphora erythrocephala; Musc.: Myospila, Pollenia; Oscin.: Oscinis coxendix; Seps.: Sepsis.

Lepidoptera (1)-Nymphal.: Vanessa atalanta.

Hemiptera (1)—Caps.: Lygus.

Salix humilis $(9: 77-8)$.-Blooms March 18-April 28; 67 visitors observed, March 21-April 21; Mis, Y.

On staminate flowers:

Long-tongued Bees (3)-Nomad.: Gnathias ovatus $q$, Nomada sayi $\widehat{\sigma}+$, Xanthidium dentariae $\widehat{\sigma}$.

Short-tongued Bees (13)-Halict. (): Chloralictus pilosus, pruinosus, sparsus, zephyrus, Evylaeus foxii, Oxystoglossa confusa; Andren.: Andrena illinoensis 0 , mandibularis $q$, salicacea $q$, type, sayi $\delta^{\lambda}$, Trachandrena elaytoniae $\widehat{\delta} q$, fq, hippotes $q$, mariae $\delta$, se.

Other Hymenoptera (1)-Chrysid.: Holochrysis hilaris.

Diptera (4)-Syrph.: Eristalis aeneus, transversus, Orthoneura nitida; Conop.: Myopa vesiculosa.

Lepidoptera (1)-Nymphal.: Euvanessa.

Coleoptera (2)-Coccinell.: Hippodamea parenthesis ; Lampyr.: Ellychnia.

Hemiptera (2)—Caps.: Lygus ab; Pentatom.: Euschistus variolarius.

On staminate and pistillate flowers:

Long-tongued Bees (2)-Ap.: Apis sc, ab; Megachil.: Ceratosmia $0^{7}$.

Short-tongued Bees (12)-Halict. (q): Chloralictus versatus, fq, Curtisapis forbesii, Evylaeus arcuatus se, fq, Halictus lerouxii ab; Collet.: Colletes inaequalis $\delta \uparrow, a b$; Andren.: Andrena carlini

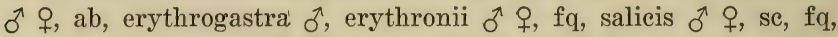
Opandrena cressonii $\delta^{\lambda}, \mathrm{fq}$, miserabilis $\delta^{\lambda} q$, fq, Trachandrena forbesii $0^{\top}+$, fq.

Diptera (12)—Syrph.: Brachypalpus frontosus ab, Eristalis dimidiatus ab, Helophilus similis ab, Sphaerophoria, Syrphus americanus; Tachin.: Gonia capitata ab; Sarcophag.: Cynomyia ab; Musc.: Phormia regina, Pseudopyrellia ab; Anthomy.: Phorbia platura ab; Scatophag.: Scatophaga ab; Seps.: Sepsis ab.

Coleoptera (1)-Chrysomel.: Orsodachna ab.

On pistillate flowers:

Long-tongued Bees (2)-Bomb.: Bombus impatiens q; Ceratin.: Ceratina $\hat{\sigma}, \mathrm{fq}$. 
Short-tongued Bews (4)-Hulict. (9)-Agapontenum mailinfus, Seladonia; Sphecodini: Sphecodes arvensis; Andres.: Audrwa illinoensis of.

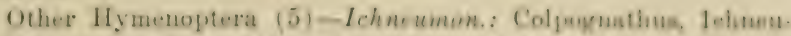
mon funestus; Tenthredin.: Dolerus arvensis ab, bicolor ab, novisous.

Diptera (2)-Syrph.: Eristalis latifrons; Anthomy: Ifycio. dosia.

Hemiptera (1)-Lygae.: Lygaeus.

Salix longifolia.-Bloms April 26-August 11: 119 viutors observed on staminate flowers, April 26-July 15; Mis, Y.

Iong-tongued Bees (16) - Ip.: Apis: Bomb.: Bombins amri.

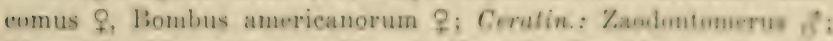
Meguchil: Megachile mendicat of; Nomud.: Gnathias wunestus ?. fq, ovatus of \&, ab, Heminomada of 9 , fq. Holonomadn superta of.

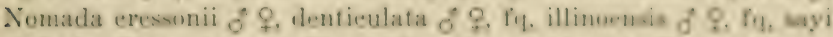

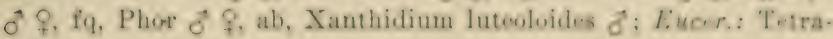
lonia dilecta $q$.

Short-tongued Bees (43)-Halict.: A frapostomon raliatus 9. se, splendens ?. Chloralietus cresonii ?, nymphuearum ?. pilosus o q. sparsus \&, se. fq, tegularis \&, se, versatus \&, sc. fq, mphyru

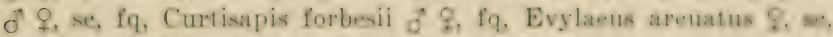

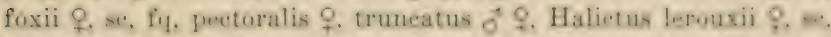
Odontalietus \&, se, Oxystoglosa enfusa of ?. purn \&. similis 8, wo. Seladonia \&. se; Sphemelini: Sphenendes arvensis ?, minor Q. Sphecodium eressunii f; Prosopial.: Prowopis illinoensis 9. Pọ. pyomens

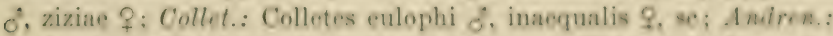
Andrena earlini \&, erythrogastra of \&, se, ab, illinoensis $q, a, f_{1}$,

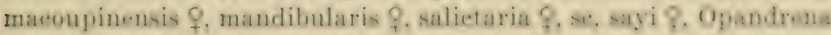
crestonii of \&. sc. fq, miserahilis of f, se, personate $d$, Parnodrena

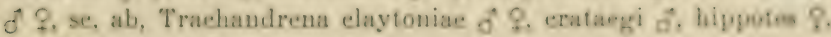
se, ab, mariae ơ $q$, se, fq.

Other IIymenoptern (10) - Sphec: Sphes nigrions: Srobl: Elis S-eineta; Philanth.: Cereris finitima, Ihilanthus puovtatum $f_{4}$ : Vesp.: Polistes pallipes, variatus: Crabren.: Lindenins orrans: Orybel.: Notoglosa frontalis, Oxyhelus packarili; TCAmenemen: Pimpla notanda.

Diptera (47)-Bibion: Libio femoratos, pellipas; Bimulf: Simulium; Taken,: Chrysops striatus; Strakamy: Nenenthes: Syrph.: Allograpta, Bacesha auriuota, Chiliela copillata, punotelate, Chryeochlamys. Chrysogater nieripes. Erotalis dimidlatus

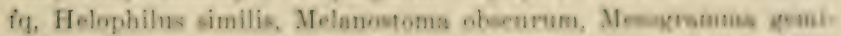

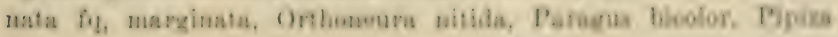


pulchella, Platychirus quadratus, Sphaerophoria fq, Syritta, Syrphus americanus; Empid.: Empis avida, distans, Parempis, Rhamphomyia limbata fq, priapulus fq, Tachydromia; Bombyli.: Bombylius major; Conop.: Myopa pilosa, vesiculosa, Zodion fulvifrons; Tachin.: Euphorocera claripennis, Gonia capitata, Linnaemyia, Peleteria, Phorantha humeralis; Sarcophag.: Cynomyia fq, Helicobia helicis, Sarcophaga cimbicis, sinuata; Musc.: Pseudopyrellia; Anthomy.: Coenosia "antica, Phorbia platura fq; Ortal.: Chaetopsis; Scatophag.: Scatophaga.

Lepidoptera (1)-Lycaen.: Eupsyche.

Coleoptera (2, fq)-Chrysomel.: Diabrotica vittata; Coccinell.: Megilla in cop.

Salix nigra.-Blooms April 22-May 29; 97 visitors observed, April 22-May 29; Mis, Y.

On staminate flowers:

Long-tongued Bees (14)-Ap.: Apis se, fq; Bomb.: Bombias separatus $q$, Bombus americanorum $q$; Ceratin.: Ceratina ot, Zaodontomerus $\delta$; Megachil.: Ceratosmia + , se; Nomad.: Gnathias

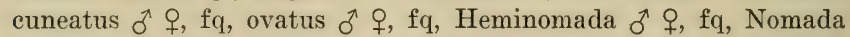
cressonii $\delta \hat{q}$, denticulata $\hat{\delta} q$, illinoensis $\delta \hat{q}$, fq, sayi $\sigma^{\lambda} q$, Phor 오.

Short-tongued Bees (43)-Halict. (q):-Agapostemon radiatus se, Augochlora viridula, Chloralictus pilosus se, fq, pruinosus sc, sparsus se, ab, tegularis se, versatus se, ab, zephyrus sc, ab, Curtisapis forbesii se, Evylaeus arcuatus sc, foxii se, fq, pectoralis se, 4-maculatus, Halictus lerouxii se, Odontalictus se, Oxystoglossa confusa se, fq, similis fq, Paralictus cephalicus, Seladonia se; Sphecodini: Machaeris stygia; Collet.: Colletes inaequalis $q$; Andren.: Andrena carlini $q$, dunningii $q$, se, erythrogastra $o^{\lambda}+$, se, ab, illinoensis $\delta^{\lambda} q, \mathrm{se}, \mathrm{fq}$, macoupinensis $q$, se, type, mandibularis $q$, se,

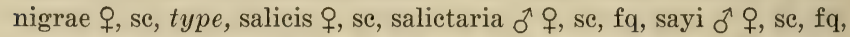
Opandrena cressonii $\delta^{\pi}+, \mathrm{sc}, \mathrm{ab}$, dubia $\delta^{\lambda}$, miserabilis $\delta^{\lambda}+, \mathrm{sc}, \mathrm{ab}$, personata $\delta^{\lambda}$, Parandrena $\delta^{\lambda}+$, se, ab, Trachandrena claytoniae $q$, sc, crataegi $\delta^{\lambda}+$, sc, ab, forbesii $\sigma^{\lambda}$, heraclei $q$, sc, hippotes $q$, se, nuda $q$, se, rugosa $q$, se.

Other Hymenoptera (3)-Eumen.: Ancistrocerus tigris; Chrysid.: Holochrysis hilaris; Bracon.: Microdus fq.

Diptera (32)-Bibion.: Bibio albipennis, pallipes; Syrph.: Allograpta, Chilosia capillata, punctulata, Chrysogaster nigripes, Eristalis aeneus, dimidiatus, Helophilus similis, Mesogramma marginata, Psilota, Sphaerophoria, Syritta, Syrphus americanus, ribesii; Empid.: Rhamphomyia limbata, priapulus fq, sordida; Bombyli.: 


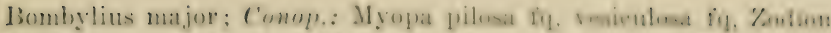
fulvifons: Twhin.: Arohytas analis. Civmosoms. Hypostone

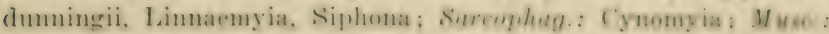

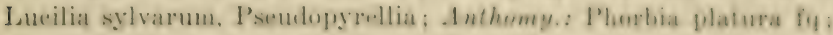
Scatophag.: Seatophaga.

Lepidoptera (2)-Nymphal.: Vanessa huntera; I'upilion.: Eurymus.

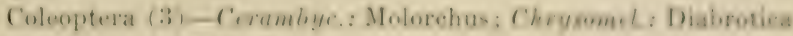
vittata; Lampyr.: Ellychnia.

\section{SANTAIACI:AI:}

Comandra umbellata $(6: 50-1)$. Lilonms April 14 . Ium 6; 41 visitors observed, May 1-23; Pol, W.

Long-tongued Bews (4)-.1p.: Apis: Megachil.: Onin pumbla Q: Nomal.: Holonomadat superba of f $_{\text {: Eucer.: Tetralunia dileeta }}$.

Short-tongued Bees (8. f)-Mulit.: Chloralietus alhip-nuis. terularis, versatus. Halietus lerouxii, Sphecolini: Spheomirs arvert. sis; Amlren.: Andrena siyi, Opandrena miwrabilis, Trachamirema mariae.

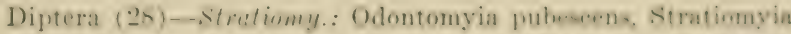
quaternaria; Syrph.: Eristalis dimidiatus. Helophilus latifrum. Sphaterophoria ab, Syritta, Tropiclia manillata, Voluwella vese culosa: Tarhin.: Conia eapitata. Peleteria. Tachina robusta

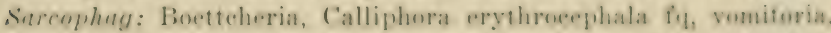
Cynomyia ab, Sareophaga cimbieis ab, simuata; Muse.: Cirapho myia. Lueilia eaesar ab, sericata fy, sylvarum ab, Myospila, Phormia regina fq. Pseudopyrellia: . Anthomy.: Coenosia antiea, I imouphera ab, Phorbia platura ab; Sciomyz.: Seiomyza.

Lepidoptera (2)-Lyaten. Cranotes: Papilonn. Iphielides

Coleoptera (2)-Coccinell.: Mecrilln; Lempyr.: Telephuvus bilineatus.

\section{SAPIND.ICE:AE}

Aesculus glabra $(16: 160)$.-Blooms April 7-May 18, 9 vintors observed, April 21-May 11; Ma, I.

Long-tongued Bees (9)-Bomb. (q): Bombias aurieomus fy. coparatus, Bumbus americanorum fig, impatiens: Megorhd: C'en. tromia $\delta$ ?. s\%. fq, O-mia cordata $\delta$ : Eveer: Tetralonia helfrapei of $q$, se, f(g, in eop), dilecta + ; Anthophor.: Anthophora \&. se.

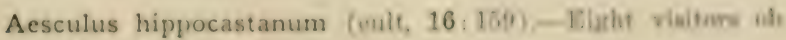
served, May 29; Ma, W.

Birds (1)-Trochil.: 'Trochilus. 
Long-tongued Bees (7)-Bomb. (q): Bombias auricomus ab, seutellaris, separatus ab, Bombus americanorum ab, impatiens $a b$;

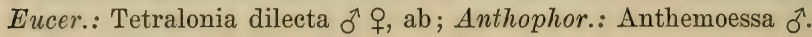

\section{Saxifragaceae}

Heuchera hispida (5:178-9).-Blooms May 4-July 4; 6 visitors observed, May 8-June 23; Mi, Y.

Long-tongued Bees (1)-Bomb.: Bombus americanorum $q$.

Short-tongued Bees (5)-Halict. ( $(+)$ : Chloralictus pruinosus, versatus se, fq, Oxystoglossa confusa se, similis se, fq; Collet.: Colletes aestivalis $\delta^{\lambda}+$, sc, ab, oligolege.

Hydrangea arborescens $(5: 269-70)$.-Blooms June 21-August 2 ; 34 visitors observed, June 27, 30; Mis, W.

Long-tongued Bees (4)-Bomb.: Bombias separatus $\Varangle, c, a b$, Bombus americanorum ${ }^{\circ}$, c; Ceratin.: Ceratina $\uparrow$, se; Megachil.: Neotrypetes truncatus $q$, c.

Short-tongued Bees (8)-Halict.: Chloralictus sparsus ${ }^{\text {, }}$ sc, $\mathrm{ab}$, versatus $q, \mathrm{se}, \mathrm{ab}$, Evylaeus areuatus $q$, se, pectoralis $\sigma^{\top} q$, se, truncatus $q$, sc, Oxystoglossa pura $q$, se, Seladonia $q$, c; Prosopid.: Prosopis ziziae $\delta^{\lambda} q, \mathrm{sf}, \mathrm{ab}$.

Other Hymenoptera (1)-Crabron.: Solenius.

Diptera (14)—Syrph. (sf) : Allograpta, Eristalis tenax, Mesogramma geminata, Paragus tibialis, Sphaerophoria, Syritta, Syrphus americanus; Empid.: Parempis ab; Conop.: Oncomyia fq, Stylogaster biannulata; Tachin.: Archytas analis; Musc.: Graphomyia, Musca, Pseudopyrellia.

Lepidoptera (2)-Hesperi.: Epargyreus; Pyromorph.: Harrisina.

Coleoptera (5)-Cerambyc.: Euderces sf, Typocerus velutinus; Mordell.: Mordella marginata ab, Mordellistena ornata, pubescens ab.

Philadelphus grandiflorus (cult, 5:270).-Twenty-one species and 160 individuals; May 29-June 26; Mi, W.

Long-tongued Bees (2:67)-Bomb.: Bombus americanorum ㅇ, 1; Megachil.: Prochelostoma philadelphi $\delta^{\lambda}+$, sc, in cop, 66, type.

Short-tongued Bees (8:58, q)-Halict.: Chloralictus sparsus sc, 32, versatus se, 5, zephyrus 1; Prosopid.: Prosopis ziziae 2; Andren.: Opandrena cressonii se, 1, personata 4, Trachandrena claytoniae se, 9 , erataegi se, 4 .

Other Hymenoptera (2:4)-Eumen.: Ancistrocerus tigris 1, Leionotus foraminatus 3 . 
Diptera (8: 27)-Bibion.: Dilophns obewulus 2: Muke: Luwdia catesar 1, sericata 1. Pollenia 2, Psoudopyrellia 2; Anthomy : Limnophora 1 , Phorbia aera 17 , platura 1.

Lepidoptera (2:4)-Papilion.: Jasoniades 1; Merperi: Epar. gyreus 3.

Ribes gracile $(5: 270-1),-$ Blooms Mareh 30-May 16; 34 vin. itors observed, April 4-29; $\mathrm{Ma}, \mathrm{W}$.

Long-tongued Bees (18) $-A_{\gamma}$.: Apis se, ab, n; Bomb. (9) : Bombias auricomus ab, suparatus, Bombus amerieanorum ab, consimilis, inuatiens ab, ridingsii; Meguehil. (d, n) : Ceratomis, Osmia pumila; Nomud. (n) ; Gnathias euneatus d, ovatus $\delta$, Nomanda dentieulata $\delta$. illinonsis of sayi $\delta$ ? T. Xanthidium lutoolotdes $\delta$. luteolum of \&. fig; Eueer.: Tertralonia dileeta of ab; Anthegphor: Anthophora $\delta$.

Short-tongued Bees $(17, \mathrm{n}\}$-IIUlict. (Q) : Agrapostemon radis. tus, Anguehlora viridula, Chloralietus eaeruleus, eremonil, sparsus sc, zephyrus, Curtisapis euriacea, Evylaens foxii sf, ab, Hallotus lerouxii sf. Oxystoglossa confusa: Collet.: Colletes aretivalis o \&:

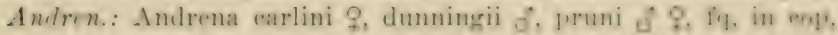
sayi $\delta^{\top}+$, ab, Trachandrena claytoniae $\delta$, rugosa $\delta$.

Diptera (1)-Bombyli.: Bombylius major $\mathrm{n}$.

Lopideptera (2)-Lyeaen.: Cyaniris n; Pupilion.: Tphielides.

\section{Scropiumariaceate}

Castilleja coccinea $(13: 595)$ - - Blnoms April 27 Jume $10 ; 1$ visitor observed, May 5, 19; Ma (O), R.

Birds (1)-Trochil.: Trochilus.

Collinsia verna $(13: 545-90)$.-Blooms April 21-June 1; 26 visitors observed, April 21-May 8; Ma, R.

Long-tongued Bees (18)-Ap.: Apis sc. ab; Bomb. (8) : Bombias auricomus, scutellaris, separatus, Lombus amerieansanu, im. patiens, ridingsii; Cerclin.: Ceratina of \&. fic, Zandentamerus of: Megachil. $(\delta$ \& sce) : Ceratosmia ab. Diecratomin ab, Gowin atri. ventris ab, eollinsiae fy, type, pumila ab; Nomed.: Nomala way $\xi$ :

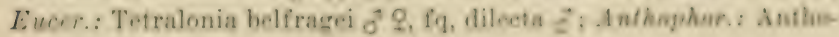
phora $\sigma^{\rightarrow}$ ?

Short-tongued Bees (1)-IInlict.: Halictus lerouxii o. c.

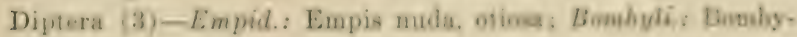
lius major.

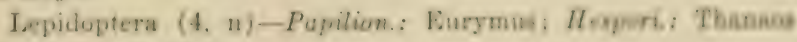
brizo, icelus, persius. 
Gerardia auriculata (13:597).-Blooms August 26-September $28 ; 7$ visitors observed, August 30-September 12; Ma, R.

Long-tongued Bees (6)-Ap.: Apis; Bomb. (ఫ) : Bombias auricomus, Bombus americanorum sc, ab, impatiens sc; Eucer.: Melissodes boltoniae $q$, c; Megachil.: Megachile brevis $q$, se.

Short-tongued Bees (1)-Halict.: Chloralictus versatus $q$, esp.

Gerardia grandiflora (13:594-5).-Blooms July 29-September 20 ; 11 visitors observed, August 8-September 14; Ma, Y.

Birds (1)-Trochil.: Trochilus.

Long-tongued Bees (7)-Bomb.: Bombias auricomus $\Varangle$, c, separatus $\Varangle, c$, Bombus americanorum $\delta^{\Uparrow} q \not{q}, \mathrm{se}, \mathrm{ab}$, consimilis $\not{q}$, impatiens $\Varangle$, sc; Megachil.: Megachile mendica ${ }^{2}$, c; Eucer.: Melissodes bimaculata $\widehat{\gamma}$, + , se.

Short-tongued Bees $(2, q, \mathrm{n})$ : Halict.: Chloralictus versatus csp, Curtisapis forbesii + , c.

Diptera (1)—Syrph.: Pipiza nigribarba $f, n$.

Gerardia purpurea (13:595-6).-Blooms August 30-September 29 ; 5 visitors observed, September $4-26$; Ma, R.

Long-tongued Bees (5, sc)-Bomb.: Bombus americanorum ठ 우 ఛ, ab, impatiens $\Varangle ;$ Megachil.: Megachile brevis $q$; Eucer.: Melissodes boltoniae $q$, nivea $q$.

Gerardia tenuifolia (13:596-7).-Blooms August 19-October 16 ; 17 visitors observed, August 26-September 23; Ma, R.

Long-tongued Bees (10)-Ap.: Apis se, ab; Bomb. (sc) : Bombias scutellaris $\Varangle$, separatus $\Varangle$, Bombus americanorum $q \not{q}, \mathrm{ab}$, impatiens $\not$, ab; Ceratin.: Ceratina $q$; Eucer.: Melissodes boltoniae q, c; Megachil. (q) : Megachile brevis se and cutting petals, fq, mendica e, Xanthosarus c.

Short-tongued Bees (3)-Halict.: Chloralictus versatus $q$, esp, $\mathrm{n}$, Oxystoglossa confusa $\hat{\sigma}, \mathrm{n} ;$ Panurg.: Calliopsis andreniformis $\delta^{7}$, se, ab, in cop.

Diptera (1) -Syrph.: Syrphus americanus fsp, n.

Lepidoptera (2, n)-Papilion.: Eurymus, Pieris.

Coleoptera (1)-Melo.: Pyrota mylabrina gn, n.

Gratiola virginiana (13:592).-Blooms May 11-July 17 ; 1 visitor observed, June 2; Mi, W.

Short-tongued Bees (1)-Halict.: Chloralictus versatus $q$, sc, ab.

Ilysanthes dubia.-Blooms June 19-September 1; 10 species and 18 individuals observed, July 31, August 3, 5, 6; Mi, W. 
Short-tongued Bees (5: 12)-Haliet.: Chimalietus oberurus 8.

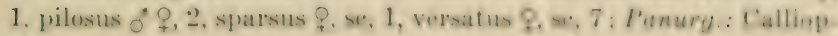
sis andreniformis $q, 1$.

Lepidoptera (5:6)-Lyeaen.: Everes 1; P'apilion.: Eurema 2, Eurymus 1; Mespuri.: Aneyloxypha 1, Pholisova hayhursil 1.

Linaria vulgaris (iut, 13 : 555).-Blooms May 1-thetober 24, 12 visitors observed, June 25-Oetober 10; Ma, I.

Long-tongued Bees (6)-Ap.: Apis se, n; Bomb.: Bombus americanorum \& \& \&. se, ab, consimilis 8 , impations b, so: $Y_{6}$. gachil.: Aleidamea simplex + , se, n, Megachile brevis $q, e, n$.

Short-tongued bees $(1)$-Malid.: Agapostemon virdulus 8. u.

Lepideptera (5, n)-Nymphal.: Vanrea eardni; Popilone: Eurymus, P'ieris; Hesperi.: Limochores tammas, Thanaus juwenalis

Mimulus alatus $(8: 145)$.-Blooms July $1: 3-$ September $5: 1$ visitor observed, August 3; MIa, R.

Long-tongued Bees $(1)$-Bomb.: Bombus amerieanorum $Q$.

Mimulus ringens $(8: 147-8)$.-Blooms July 11 - Septemher 7 : 2 visitors observed, August 1,$13 ;$ Ma, R.

Long-tongued Bees $(2)-B o m b .:$ Bombus amerieanorum 9 o. consimilis $\not$.

Pentstemon hirsutus $(13: 591-2$ ).-Blomms May 1-June $7: 27$ visitors observed, May $1-$ June $3 ; \mathrm{Ma}, \mathrm{W}$.

Long-tongued Bees (21)-Bomb. (P): Bombias auriumus Pq, s.paratus, Bombus amerieanorum $f y$, consimilis. impatiens; Crutin.: Ceratina 9; Megachil.: Aleidamea simplex of 8. $s$, fiq. Centrosmia q. se. Ceratosmia \&. Osmia atriventris d \&. so, fig, enl.

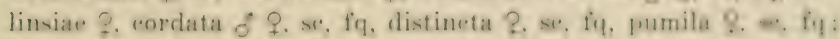

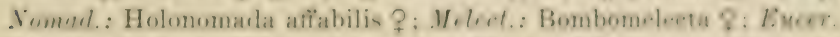

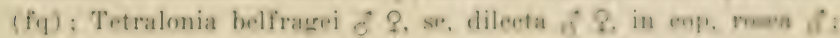

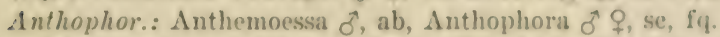

Other Iyzuenoptera 11 -Eumin.: I. ionotus foraminatos, per forating.

Diptera (1)—Bombyli.: Bombylins atriceps.

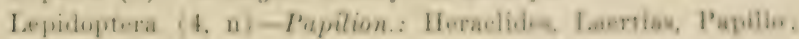
IIesperi.: Atrytone zabulon.

Pentstemon laevigatus $(13: 590-1)$ - Mlown Ms) 16-July $9:$ 30 visitors observed, May 28-June 26; Ma, W.

Birds (1)-Trochil.: Troehilus.

Long-tongued Bees $(17)-A p .: \Lambda_{p}$ is; Bomb.: Bombias auri-

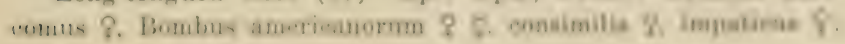


Ceratin.: Ceratina $ᄋ$, c, n; Megachil.: Osmiini : Alcidamea simplex $\delta^{\lambda}+$, se, ab, Monilosmia $q$, Osmia distincta $q$, se, fq, pumila $q$;

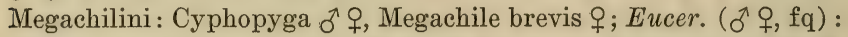
Tetralonia dilecta, rosae; Anthophor. (sc): Anthemoessa 0 \% $\mathrm{fq}$, Anthophora $q$, fq, Clisodon $q$.

Short-tongued Bees (5)-Halict. ( $(9, \mathrm{c}, \mathrm{n})$ : Agapostemon viridulus, Chloralictus pilosus, pruinosus, versatus, Curtisapis coriacea.

Other Hymenoptera (2, n)-Eumen.: Leionotus foraminatus, perforating, anormis, at holes.

Diptera (1)—Syrph.: Mesogramma marginata fsp, n.

Lepidoptera $(3, \mathrm{n})-$ Papilion.: Euphoeades, Eurymus; Sphing.: Deilephila.

Coleoptera (1)—Scarabae.: Trichius piger $\mathrm{f}, \mathrm{n}$.

Scrophularia marilandica (13:586-8).-Blooms July 8-October 26 ; 39 visitors observed, July 12-September 19; Mi, R.

Birds (1)-Trochil.: Trochilus fq.

Long-tongued Bees (7)-Ap.: Apis ab; Bomb.: Bombus consimilis $\sigma^{\top} \not$, ab, impatiens $\not$, se, ab; Ceratin.: Ceratina ${ }_{+}$; Megachil.: Anthemois $\delta$, Cyphopyga + ; Eucer.: Melissodes bimaculata o $q$, fq.

Short-tongued Bees (9)-Halict.: Agapostemon viridulus $\sigma^{\pi}$, Augochlora viridula $\delta^{\lambda}$, Chloralictus sparsus $\delta \rho, f q$, versatus $\delta^{\lambda}+$, sc, ab, zephyrus $\delta$, ab, Curtisapis coriacea $\delta^{\lambda}+$, sc, Halictus lerouxii $q$, sc, Oxystoglossa confusa $\delta^{\uparrow}+$, se, fq, Seladonia $\delta^{1} q$ sc.

Other Hymenoptera (17)—Sphec.: Sphex vulgaris; Philanth.: Philanthus punctatus; Vesp.: Polistes annularis, pallipes, rubiginosus, variatus, Vespula cuneata, germanica ab, maculata $a b$; Eumen.: Ancistrocerus campestris, Eumenes fraternus fq, Leionotus anormis, foraminatus, scrophulariae, ziziae, Monobia, Zethus.

Diptera (3)—Syrph. (f, n) : Mesogramma marginata, polita; Tachin.: Siphona.

Lepidoptera (2)-Lycaen.: Everes; Ctenuch.: Scepsis.

August 6,14 , the following 8 species and 26 individuals were taken on the flowers :-

Short-tongued Bees (3:21)-Halict.: Chloralictus sparsus 5, versatus 7 , zephyrus 9 .

Other Hymenoptera (4:4)-Vesp.: Polistes annularis 1, pallipes 1, variatus 1, Vespula maculata 1 .

Diptera (1)-Tachin.: Siphona 1.

Seymeria macrophylla (13:593-4).-Blooms July 13-August $22 ; 9$ visitors observed, July 28-August 8; Ma, Y. 
Long-tongued Bees (6) -1 p.: $\Lambda$ pis; Romb. (8) : Isombius anon. icanorum se, ab, consimilis sa, ah, impatiens, ridingdi wo; $I$ iscer: Melissodes bimaculata $\sigma^{7}$.

Diptera (1)-Syrph.: Milesia f, n.

Lepidoptera (2, n)-Papilion.: Pieris; Hesperi.: Atrytone zabulon fq.

Verbascum thapsus (int, $13: 583-4$ ),-Blooms June 4-0etobe? $26 ; 16$ visitors observed, June 27 -August $15 ;$ Мa, Y.

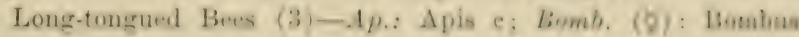
amerieanorum e, consimilis se.

Short-tongued Bees (6)-Malict. (9, e): Agapmetemon wirilulus,

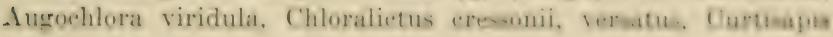
coriacea, Evylaeus pectoralis.

Diptera $(7, f, n)-$ Syrph.: Allograpta, Mesngramma gesninata, marginata, Pipizal nigriharba, Syritta, Syrphus amerweanus: Anthomy.: Phorbia platura.

Veronica arvensis (int),-Blooms April 12-June 12: 3 visiton observed, May 3, 19; Mi, R.

Short-tongued Bees (2)-Hulict. (q): Chlovalictus seratus 3, Oxystoglossa similis 1.

Diptera (1)—Syrph.: Mesogramma marginata 4.

Veronica peregrina.-Blooms Amil 9-Jume 20: 13 visitom ob. served, May $3-8 ; \mathrm{Mi}, \mathrm{W}$.

Short-tongued Bees $(8,9)$-Hulict: Chloralietus pilnsus, juruinosus, sparsus, versatus fiq, zephyrus. Oxystoglowa similis fo, Seladonia fq; Andren.: Opandrena cressonii.

Diptera (4)—Syrph.: Mesogramma marginata, Sphaeroglawia : Anthomy.: Phorbia acra; Oscin.: Oscinis soror.

Hemiptera (1)-Corimelaen.: Corimelaena puliearia.

Veronica virginica (13:592-3).-Blows Juse 20-Augast 27: 42 visitors observed, July 7 -August 11; Mis, W.

Long-tongued Boes $(9)-.1$ p.: Apis ab; Bamb. (6): Barahue am.rieanorum, consiuilis; Crrutim. Coratina O. we Megechef.: Trypetini: Neotrypetes truncatus ?. e; Omini: Alefiames aim.

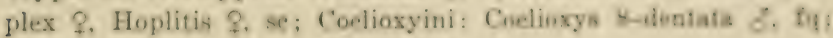
Eucer.: Melissodes bimaculata ơ $q$, f $q$.

Short-tongued Bees (13)-Haliet.: Agapontemon tesauma \&. viridulus of \&. Angochlora fervida \&. Chlowalictus pilomus \&. wpanos

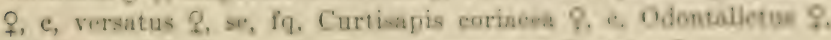
Selardonia of $q$ : Prosopicl. Prosopis pyemeen \&, niziae? 
Other Hymenoptera (4)-Sphec.: Ammobia ichneumonea, Sphex nigricans, procera; Bembic.: Stictia.

Diptera (8)-Syrph.: Eristalis aeneus sf, tenax sf, transversus f, n, Mesogramma marginata f, n, Syritta; Bombyli.: Exoprosopa fasciata; Conop.: Oncomyia; Tachin.: Archytas analis.

Lepidoptera (7)-Nymphal.: Vanessa huntera; Lycaen.: Chrysophanus, Everes; Papilion.: Pieris; Ctenuch.: Scepsis; Pyraust.: Loxostege, Nomophila.

Hemiptera (1)—Lygae.: Lygaeus.

\section{SolanACEAE}

Datura stramonium (int).-Blooms June 4-October 26; 2 visitors observed, June 25, 27; Ma, W.

Lepidoptera (2)_Sphing.: Philampelus, Protoparce.

Datura tatula (int, 13:582).-Blooms May 26-October 28; 10 visitors observed, July 13-October 28; Ma, W.

Birds (1)-Trochil.: Trochilus.

Long-tongued Bees (1)-Ap.: Apis c, ab, n.

Short-tongued Bees (1)-Halict.: Chloralictus versatus $q, \mathrm{c}, \mathrm{n}$.

Diptera (4, f, n)—Syrph.: Allograpta, Mesogramma marginata, Rhingia, Syrphus ribesii.

Lepidoptera (2)—Sphing.: Deilephila, Protoparce.

Coleoptera (1)-Chrysomel.: Diabrotica 12-puctata f, n.

Physalis lanceolata $(8: 146)$.-Blooms May $12-$ October $3 ; 5$ visitors observed, May 12-September 29 ; Mi, Y.

Long-tongued Bees (1)-Anthophor. Anthophora q.

Short-tongued Bees (4)-Halict.: Chloralictus versatus o, c; Collet.: Colletes latitarsis $\delta^{\wedge} q$, sc, ab ; willistonii, $\sigma^{\lambda} q, \mathrm{sc}, \mathrm{fq}$, type; Panurg.: Zaperdita $\sigma^{\lambda}+$, se, ab.

Physalis subglabrata $(8: 146-7)$.-Blooms July 12-September 27 ; 1 visitor observed, July 27; Mi, Y.

Short-tongued Bees (1) -Collet.: Colletes latitarsis o, sc.

Physalis virginiana $(8: 146)$.-Blooms June 7 -October $4 ; 5$ visitors observed, June 19-August 1; Mi, Y.

Short-tongued Bees (5)-Halict. ( $q$, sc) : Evylaeus pectinatus, Oxystoglossa confusa; Collet.: Colletes latitarsis ot $q$, sc, ab, type, willistonii + , se ; Panurg.: Zaperdita ot $q$, se.

Solanum carolinense $(13: 582)$._-Blooms June 4-October $7 ; 3$ visitors observed, June 25-August 29 ; Ma, W.

Long-tongued Bees (3, ఫ, c)-Bomb.: Bombias seutellaris, Bombus americanorum, impatiens. 
Solanum nigrum (13:582).-Blooms June 6-Detober 20 ; 2 vis itors observed, August 29, September 3; Ma, W.

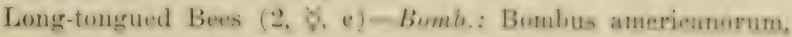
impatiens.

Solanum rostratum (int).-Blooms ofuly 9- Oetoler 4; 1 vinitor observed, July 23 ; M a, Y.

Long-tongued Bees (1)-Bomb,: Bombns americanorum 6. e.

\section{Stapiyluacha:}

Staphylea trifolia $(14: 302-3)$.-Blooms April 23-May 12: :21 visitors observed, April 23-May 11; Mi, W.

Lone-tongued Bees $(4)-A$ p.: Apis sa, ab; Romb. $(8, a b)$ : Bombus amerieanorum, consimilis, impatiens.

Short-tongue Bees (9)-Halict. (q): Curtisapis coriacea, Evylaeus 1-maculatus, Scladonia; Collet.: Colletes inaequalis of:

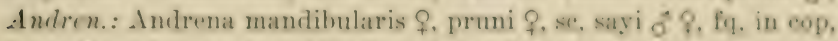
type, Opamirena miserabilis \&. Trachandrena nuila 오. fupe.

Other Hymenoptera (1)-Vesp.: Vespula maeulata.

Diptera $(4)$ Syrph.: Eristalis flavipes f: Empid.: Empis otion ab, Pachymeria; Bombyli.: Bombylius major.

Lepidoptera (1)-Hesperi.: 'Thanaos juvenalis.

Coleoptera (2)-Corambyc.: Molorehus; Scarabat: Euphoris sepulchralis.

Hemiptera (1)—Caps.: Lygus.

\section{Thymetafaceat}

Dirca palustris $(0: 7:-4)$. - Blooms Mareh $18-\Lambda$ pril $: 0$; 11 vis. itors observed, Mareh 21; Mi, Y.

Lone-tongued Bees $(4.8)$-Coralin.: Ceratina, Zaodontomerus; Megachil.: Ceratosmia; Nomad.: Gnathias cuneatus.

Short-tongned Bees (6)-Muliet. (Q): Chloralietus smilameiuses. versitus, zephyrus, Oxystoglossa pura: Collet.: Colletes innegualis ơ; Andren.: Trachandrena rugosa $\delta^{*}$.

Lepidoptera (1)-Nymphal.: Euvanessa.

\section{THIACEak}

Tilia americana.-Blooms Jume 4.July 12; 29 ageries and 180 individuals observed, July 2, 6, 7; Mi, Y.

Long-tonguid Bees $(2: 37)-1$ p.: Apih 36: Bamb: Thmina americanorum $\not{q}, 1$.

Short-tougued Bees $\{11: 61\}$-Maliet.: Aucoehlore viridali

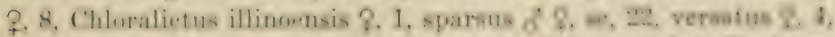

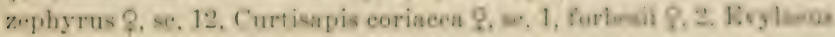


pectoralis $\delta^{\lambda}+, 2$, Halictus lerouxii $q, 1$, Oxystoglossa confusa $q, 1$. Other Hymenoptera (3:3)-Sphec.: Ammobia ichneumonea 1; Scoli.: Elis 5-cincta 1; Eumen.: Leionotus foraminatus 1.

Diptera (11:27) -Syrph.: Allograpta 2, Orthoneura nitida 1, Syrphus americanus 2; Tachin.: Archytas aterrima 1, Peleteria 1; Sarcophag.: Calliphora erythrocephala 1; Musc.: Graphomyia 1, Lucilia caesar 4, Phormia regina 1, Pseudopyrellia 11; Anthomy.: Phorbia acra 2.

Lepidoptera $(1: 1)$-Ctenuch.: Scepsis 1.

Coleoptera (1:1)-Malach.: Anthocomus 1.

\section{UMBELLIFERAE}

Anethum graveolens (Mis, Y: cult).-July 12-16, 1920, 1000 individuals taken on the flowers showed:

Long-tongued Bees (2)-Ap. 1, Megachil. 1.

Short-tongued Bees (635)-Halict. $\delta^{\lambda}+$, mostly $q$ sc., 585, Prosopid. $\delta^{\top}$ ㅇ, 50 .

Other Hymenoptera (43)-Oxybel. 19, Cercer. 12, Scoli. 4, Bracon. 3, Larr. 2, Eumen. 1, Nysson. 1, Sphec. 1.

Diptera (292)-Syrph. 246, Musc. 27, Sarcophag. 7, Anthomy. 4, Tachin. 4, Oscin. 2, Mycetophil. 1, Seps. 1.

Hemiptera (25)-Caps.

Coleoptera (3)-Fam. ? 1, Mordell. 2.

Chaerophyllum procumbens (12:459-60).-Blooms April 6May $26 ; 49$ visitors observed, April 16-May 4; Pol, W.

Long-tongued Bees (4)-Megachil.: Ceratosmia $q$, Osmia pumila o, Nomad.: Heminomada ${ }^{\lambda}$, Nomada sayi t type. $^{2}$

Short-tongued Bees (12)_Halict. (ㅇ): Chloralictus cressonii, obscurus, sparsus, versatus, Evylaeus 4-maculatus type; Halictus lerouxii fq, Oxystoglossa confusa, pura; Sphecondini: Proteraner $\delta^{\top}$; Andren.: Andrena carlini $q$, nasonii $q$, se, Opandrena ziziae $\delta^{7}$.

Other Hymenoptera (4)-Chalcid.: Chrysocharis oscinidis; Ichneumon.: Gambrus albonotatus, apicatus, Pimpla marginata.

Diptera (23)-Simuli.: Simulium; Syrph.: Chilosia petulca, Mesogramma geminata in cop, marginata, Orthoneura nitida, Paragus bicolor, tibials, Pipiza femoralis, nigribarba, Psilota, Sphaerophoria, Syrphus americanus, torvus, ribesii; Phor.: Trineura; Sarcophag.: Helicobia helicis; Musc.: Myospila, Pseudopyrellia; Anthomy.: Phorbia acra; Seps.: Sepsis; Oscin.: Elachiptera costata, Hippelates flavipes, Ossinis soror.

Coleoptera (4)-Chrysomel.: Diabrotica vittata, Glyptina, Mantura; Anthic.: Corphyra terminalis. 
Ilemiptera (2)-Corim lown.: Corimelarna muliearia, Intoralis.

Cicuta maculata $(12: 459-60)$ - Blooms Jume 2-Septerulape $16 ; 283$ visitors observed, July 8-September 8 ; Pol, W.

Long-tongued Fees (7)-Megachil.: Mesachile hrovie d. getulans of. Sayapis pugnata $q ;$ Bemb.: Rombias auricomus of, Rombea impatiens of; $A$ p.: Apis; Eucer.. Melissodes bimaculata of

Short-tongued Bers (22)-Prosopil.: Prosopin illinownis of P.

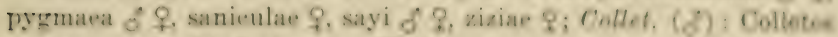
armatus, eulophi, latitarsis; Heliet.: Agapustemen radiatus of $\mathrm{S}$. viriclulus of Augoehlora fervida 8 , viridula 9 . Ohloraletus suman

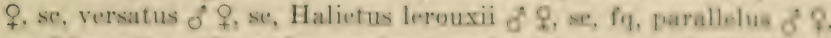
se, Odontalietus \&, se. Oxystoglosa confusu q, so, pura q. Seladonia of $q$; Sphecodini $(\mathcal{G}$ \&) : Machacris stygia. Sphecodes arveusis fog.

Other Ilymenoptera (147)-Crabron.: Anaeralion fo. Flytochrysus, Ectemnius, Hypocrabro, Lindenius errans Pụ. Proteliy. reopus rufifemur. Pseudoerabro fq. Solenius, Xestocrabro 6-mave. latus, texanus, trifaselatus; Oryblel.: Notoglosa ameriesna fọ, eressonii type. emarginata, frontalis $\mathrm{f}_{\mathrm{f}}$, inormata type. Osyledus lactus, niger, packardii fq. 4-notatus fq; Trypoxyl.: Tryposylou tridentatum; Philunth.: Cereeris bienrnuta, elypeata, comparta fo, finitima, fumipennis, kennieottii, Eureweris, Philanthus punetatus; Prmphredon.: Psen; Sphec.: Ammobia ichneumonea, peuncylvanies. Chalybion, Chlorion fy. Isodentia apiealis, macroeephala, Prionenys atrata, thomae. Seeliphron ah. Sphex gracilis, nigrieaus, vulgaris: Larr.: Astatus bicolor, unicolor fq, Larra, Larropsis, Lyroda fo. Notogonidea in. Tachysphex acutus, belfragei fig, tarsans, ter. minatus, Tachytes aurulentus $f y$, elongatus fy, mamlifularis, obseurus, peptiens, sericatus; Bembic.: Bieyrtes 4-fasiata. Jhembix spinolar, Megastizus fig. in cop, Sphecins fy, Stizus: Nysum. Hegn. lisus atricornis, simillimus, Nysson plagiatus, Zanymon aurusus. Pseudoplisus phaleratus fq : Pompil.: Ageniella longulas, Anoplins illinoensis, Arachnoprortonus, Batizonus, Ceropalms higunetata, clegans, fraterna, fulvipes, robinsonii, Cryptochellus fulgifrons. Epysiron. Lophopompilus at rox, philadelphiens fq, Pocollogompilas interruptus, navus fq. Poupiloides amerieanus. Primueusaldes fulvicornis fq. terminatus, unifasciatus, Pammoediares refativus fig, ten brosus, Sophropompilus: Tesp.: Polistes anmularis, falliprs.

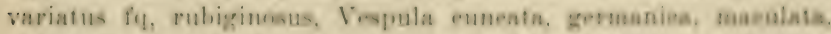

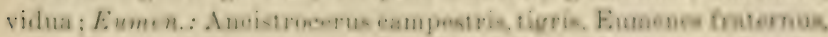
Leionotus anormis, arvensis, donalis fq, foraminatus fq. 4 socus

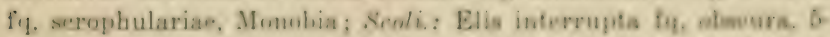

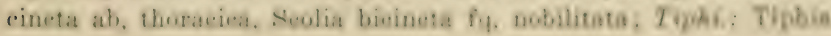


illinoensis type, transversa; Mutill. ( $\left.\delta^{\top}\right)$ : Pseudomethoca, Sphaerophthalma, Mutilla hexagona, sayi; Formic.: Formica fusea $\delta^{\star}$; Chrysid.: Gonochrysis, Hedychridium, Hedychrum wiltii, Holopyga fq, Monochrysis, Notozus, Tetrachrysis caerulans, montana, venusta ; Figit.: Eucoilidia canadensis, Figites 5-lineata, Psilodora erythropus, impatiens; Chalcid.: Chalcis, Bruchophagus, Leucospis, Perilampus triangularis, cyaneus; Evani.: Foenus tarsatorius; Ichneumon.: Ichneumon jucundus; Bracon.: Bassus, Agathis vulgaris, Orgilus, Bracon vernoniae, Microgaster, Iphiaulax.

Diptera (83)-Mycetophil.: Eugnoriste, Sciara atrata; Bibion.: Seatopse pulicaria; Taban.: Chrysops striatus, Tabanus; Stratiomy.: Nemotelus, Stratiomyia meigenii, unilimbata; Mida.: Midas clavatus, tibialis; Bombyli.: Exoprosopa fascipennis; Scenopin.: Scenopinus; Syrph.: Allograpta, Eristalis aeneus, flavipes, latifrons, tenax, Mallota illinoensis, Mesogramma geminata, marginata, polita, Orthoneura nitida in cop, Paragus bicolor fq, tibialis, Pipiza pulchella, Platychirus quadratus, Sphaerophoria fq, Syritta ab, Tropidia quadrata; Conop.: Conops brachyrrhynchus, xanthopareus, Oncomyia, Zodion nanellum; Tachin.: Alophora aeneoventris, Archytas analis, aterrima, hystrix, Paradidyma, Trichophora, Ennyomma clistoides, Clytiomyia, Exorista confinis, pyste, Cistogaster oceidua $f q$, pallasii, Gymnosoma, Belvosia bifasciata, unifasciata, Linnaemyia fq, Opsidia, Pachyophthalmus signatus (aurifrons fq, type), Phasioclista, Phorantha pruinosa, purpurascens $\mathrm{fq}$, type, Euphorocera claripennis $\mathrm{fq}$, Senotainia rubriventris $\mathrm{fq}$, trilineata $f q$, Spallanzania, Sturmia nigrita, Tachina rustica, Trichopoda lanipes, pennipes, Winthemia ; Sarcophag.: Helicobia helicis, Sarcophaga assidua, cimbicis, hunteri, sinuata ; Musc.: Compsomyia fq, Graphomyia, Morellia, Musca fq, Phormia regina, Pseudopyrellia ab, Stomoxys; Anthomy.: Calythea, Limnophora, Phorbia acra, Hylemyia laevis; Trypet.: Euaresta aequalis; Seps.: Sepsis; Oscin.: Hippelates plebejus; Agromyz.: Agromyza latipes.

Lepidoptera (8)-Nymphal.: Argynnis, Basilarchia archippus, Hypatus, Phyciodes; Lycaen.: Everes; Papilion.: Eurymus, Iphiclides; Ctenuch.: Scepsis.

Coleoptera (14)-Coccinell.: Coccinella 9-notata, Scymnus consobrinus; Cerambyc.: Batyle, Euderces; Chrysomel.: Diabrotica atripennis, 12-punctata; Bruch.: Bruchus bivulneratus; Mordell.: Mordella marginata, melaena, scutellaris; Melo.: Epicauta trichrus; Rhipiphor.: Myodites; Curculion.: Centrinus picumnus, scutellumalbum.

Hemiptera (2)-Corimelaen.: Corimelaena pulicaria ; Phymat.: Phymata, pred. 
Cryptotaenia canadensis $(12: 459-60)$.-Blooms June 1-July 17 ; 112 visitors observed, June 8-July 9; Pol, W.

Long-tongued Bees (2)-Megachil.: Neotrypetes truncatus of $q$, se; Ceratin.: Ceratina ㅇ.

Short-tongued Bees (28)-P'rosopid.: Prosopis pygmana of $q$.

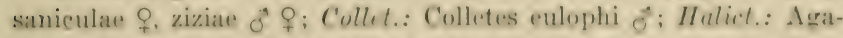
postemon radiatus of $q$. Chloralietus cacruleus de cressonii $\rho_{\text {g }}$ illino-

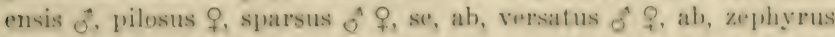
$\hat{o}$ ㅇ. Evylatus foxii of peetinatus o, pectoralis of. fq, truncatus of type. Halietus lerouxii q. Odontalietus o. Oxystoglossa confusat $q$, pura of $q . \mathrm{fq}$, Seladonia of $q$. fq; Spheeodini: Mareharris stygia of, Spheodes arvensis $q$. Sphecodium eressonii of \&. fq; Andren. (\&): Andrena platyparia, Opandrena serotina se, Trachandrena spiraeana; Panurg.: Calliopsis andreniformis $\delta$.

Other IIymenoptera (19)-C'rabron.: Anaerabro, Clytochrysus, Solenius; Oxybel.: Oxybelus niger, t-notatus, Philanth.: Cereeris astarte, elypeata; Pompil.: Ceropales fraterna, Pseudagenia; Eumen.: Ancistrocerus tigris, Leionotus peremis, Symmorphus debilis; Chrysid.: Hedyehrum parvum, violaceum; Evani.: Foenus incertus, tarsatorius; Ichneumon.: Lissonota tricineta, Polyblastus, Polysphineta.

Diptera (43)-Bibion.: Seatopse pulicaria ; Le pt.: Hilarimorpha mikii, type: Bombyli.: Anthrax sinuosa, Geron calvus; Empid.: Empis loripedis, Parempis; Syrph.: Allograpta, Eristalis transversus, Mesogramma geminata fq, marginata, Orthoneura nitida, Paragus bicolor ab, tibialis fq. Pipiza femoralis, nigribarba, Syritta fy. Syrphus americanus, ribesii fq, Volueella vesieulosa, Xanthogramma emarginata; Conop.: Oneomyia, Zodion fulvifrons, nanellum; Tuchin.: Cistogaster occidua, pallasii, Linnaemyia, Metopia, Oeyptera tenuis, Siphona fq, Xanthomelana; Musc.: Pseudopyrellia; Anthomy.: Phorbia acra, platura; Scatophay.: Cordylura; Supromyz.: Sapromyza vulgaris; Seps.: Sepsis; Drosophil.: DrosoJhila; Oscin.: Chlorops proxima, Elachiptera flavida, Hippelates flavipes, Oscinis trigramma, Siphonella inquilina.

Coleoptera (19)-Coccinell.: Seymnus terminatus; Dermerst.: Cryptorhopalum triste; Lampyr.: Podabrus brumnieollis; Moluch.: Anthowmus; Cerambye: Euderees, Typocerus lugubris: Chrysomel.: Diabrotica 12-punctata; Bruch.: Bruchus bivulneratus, exiguus, mimus fq. museulus; Mordell.: Mordella marginata, triloba, Mordellistena limbalis, puheseens, Pentaria; Rihymchit.: Eugnamptus; Ourculion.: Apion, Centrinus pieumnus.

Hemiptera (1)-Corimelacn.: Corimelaena lateralis. 
Erigenia bulbosa (12:159-60).-Blooms March 15-April 21; 63 visitors observed, March 20-April 21; Mis, W.

Long-tongued Bees (7)-Megachil. $\left(\sigma^{\lambda}\right)$ : Ceratosmia, Osmia atriventris, pumila; Ceratin.: Ceratina $\delta$, fq, Zaodontomerus $\delta$; Bomb.: Bombus consimilis $q$; Ap.: Apis sc, ab.

Short-tongued Bees (22)-Collet.: Colletes inaequalis $\delta q, \mathrm{fq}$; Halict. (q) : Agapostemon radiatus, Augochlora viridula, Chloralictus pilosus, sparsus, versatus, Curtisapis forbesii type, Evylaeus foxii, Halictus lerouxii, Oxystoglossa confusa, Seladonia; Andren.:

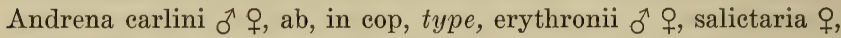
sayi $\delta$, Opandrena miserabilis $\delta$, Parandrena $\delta$ of Ptilandrena erigeniae $\delta q, f q$, type, Trachandrena forbesii $q$, mariae $\delta \uparrow$, nuda $q$, rugosa $\delta$, $f q$, in cop, type.

Other Hymenoptera (1)—Chrysid.: Holochrysis hilaris.

Diptera (29)-Tipul.: Geranomyia; Stratiomy.: Odontomyia pubescens; Bombyli.: Bombylius major; Syrph.: Brachypalpus frontosus, Chilosia capillata, Eristalis dimidiatus ab, latifrons, Helophilus similis, Melanostoma obscurum fq, Mesogramma geminata, marginata, Platychirus hyperboreus, Syritta, Syrphus americanus; Tachin.: Epalpus, Euphorocera claripennis, Gonia capitata ab; Sarcophag.: Cynomyia, Helicobia 4-setosa; Musc.: Phormia regina, Pseudopyrellia; Anthomy.: Phorbia acra, platura, Hammomyia; Lonchae.: Lonchaea aberrans, parvicornis; Scatophag.: Seatophaga; Oscin.: Elachiptera costata, Oscinis coxendix.

Lepidoptera (2)-Papilion.: Pontia; Noctu.: Plusia simplex.

Coleoptera (2)-Chrysomel.: Diabrotica vittata; Oedemer.: Asclera ruficollis.

Eryngium yuccifolium (12:459-60).-Blooms July 7-August $30 ; 182$ visitors observed, July 7-August 12 ; Pol, W.

Long-tongued Bees (13)-Megachil.: Megachilini: Megachile brevis $\delta \hat{\gamma}$, sc, mendica $\delta$, petulans $\delta$, Coelioxyini : Coelioxys octodentata $\sigma^{\lambda}+$, in cop, sayi $\sigma^{\lambda}$; Ceratin.: Ceratina $\sigma^{\lambda}$ 우 Bomb.: Bombias separatus $\delta^{\lambda} \not{\psi}$, Bombus americanorum $q$, impatiens $\Varangle ; A p .:$ Apis; Nomad.: Cephen $q$; Epeol.: Epeolus bifasciatus ${ }^{\jmath}$, Triepeolus lunatus +.

Short-tongued Bees (20)-Prosopid. ( $\overbrace{}^{\uparrow} q)$ : Prosopis pygmaea, ziziae; Collet.: Colletes speciosus + , type; Halict.: Agapostemon radiatus $\delta^{\lambda}+$, Augochlora fervida $q$, Chloralictus pilosus $q$, sparsus $q$, se, versatus $q$, se, Curtisapis forbesii $q$, Evylaeus areuatus $q$, pectoralis $q$, Halictus lerouxii $\widehat{\sigma} q$, parallelus $\widehat{0} q$, Odontalictus + , Seladonia $\delta^{1}$; Sphecodini : Machaeris stygia $q$, Sphecodes arvensis $\delta$, in cop, Sphecodium cressonii $q$; Nomi.: Paranomia $q$; Panurg.: Calliopsis andreniformis $\delta$. 
Other Ilymenoptera (5s)-Cirabron. Protothymogus rntifemur, Pseudoerabo; Oxybel.: Notorglosa americana, frontalis, ovyluelus 4-notatus; Philanth.: Cereeris bieornuta, fumipennis promineus, raui, Eucerceris, ah, Philanthus punetatus, ventilabris: Sphec: Ammobia ichneumonea, moda, penusylvaniea, Isulontia apiealis, Priononyx atrata, thomae, Sphex qrarilis, nigrieans. pietipenas in cop, vulgaris; Larr.: Astatus unicolor, Tawhysples belfragel, Tachytes aurulentus, distinetus, elongatus: Bembers: Rivorios 4. fasciata, ventralis, Bembix nubillipennis, spinolse, Mevantazus. Stizus; Pompril.: Araehnoproctumus, Coropules elegens, Iophopompilus atrox, philadelphicus. Poceilopompilos nasus. P'ung. iloides americanus, in eop. Prioenemoldes fulvicornis: Vup.: Polistes variatus; Eumen.: Ancistromerus eampurstris. Fumens belfragei, fraternus, Leionotus anormis in cop, arvensis, dowalis fy, foraminatus, vagus, Monobia; scoli.: Flis interrupta, oberura, 5. eineta, thoraciea type, Seolia bieineta, nohilitata; Mutill.: Mutilla hexagona ơ ; Chalcid.: Leucospis.

Diptera (59) -Tuban.: Tabanus; stratiem?!: Xemotolus, Odontomyia eineta; Midu.: Mislas clavatus, tibialis: Pombyli.: Anthras alternata, fulvohirta, Exoprosopa decora, fastiata, faseipennis, Geron rufipes, Systoechus; Syrph.: Allograpta, Eristalis aenems. latifrons, tenax, transversus, Mesegramma geninata, marginata, polita, Milesia, Orthoneura nitida, Paragus tibialis, Sphaepophoria, Syritta; Conop.: Conops brachyrrhynehus, xanthopareus, Oncomyia, Physocephala texana, tibialis, Zodion fulvifrons, manellum, obliquefasciatum; Tachin.: Nophora aeneoventris, Pumma, Archytas analis, Belvosia bifaseiata, Cistogaster oecidua, pallasi, Gymnosoma, Leskia, Oeyptera lata, tenuis. Phorantha asteris hrmeralis, magna, pruinosa, Senotainia rubriventris, frilineata, Spal. lanzania, Trichopoda pennipes, Trichophora; Simophag.: Bareo. phagra assidua ; Musc.: Compsomyia, Protoenlliphora, Peculozyrel. lia; Anthomy.: Coenosia antiea, Lispa uliginosa, Phortia aera.

Lepidoptera (21)-Wymphal: Anosia, Basilarehia archupros, Cereyonis, Charidryas, Phyeiodes. Speyeria: Lyoen: Chrzos. phanus, Everes, Cranotes; Papilion.: Euphueades, Luryous. Laertia, Pontia; Mesperi: l,imouhores tamuas, Pholisera catullos. Polites; Sesi.: Sesia sexfasciata; Clenuch.: Seopeis; Ardi: Lite. theisa ; Noctu.: Acontia, Spragueia.

Coleoptera (8)-Scurubue: Euphoria sepulehralis. Triobios piger; Chrysomel.: Cryptocephalus: Mrlo, Nemognarho, Iynota

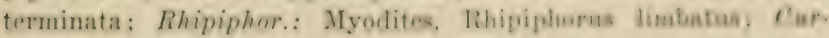
culion.: Centrinus seutellum-album.

Hemiptera (3)-Core.: Alydus eurinus; Leygur: : Lyganus, Oncopeltus. 
Eulophus americanus $(12: 459-60)$.-Blooms May 12-June 15; 130 visitors observed, May 28-June 15; Pol, W.

Long-tongued Bees (1)-Ceratin.: Zaodontomerus ô?,

Short-tongued Bees (21) -Prosopid.: Prosopis eulophi $\delta^{\wedge} q, \mathrm{fq}$, type, illinoensis $\delta^{\lambda}$, pygmaea $\delta^{\lambda}+$, saniculae $\delta^{\lambda}+$, sayi $\delta^{\lambda}+$, thaspii $q$,

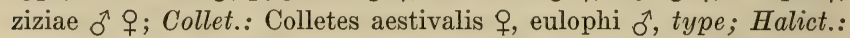
Agapostemon radiatus $q$, Chloralictus pruinosus $q$, se, sparsus $\delta^{\lambda} q$, sc, fq, versatus $q$, Evylaeus arcuatus $q$, pectoralis $q$, sc, Halictus lerouxii $q$, sc, parallelus $q$, Oxystoglossa confusa $q$; Andren.: Opandrena personata + , Trachandrena crataegi $q$, sc; Panurg.: Heterosarus $q$, se.

Other Hymenoptera (30)-Crabron.: Pseudocrabro, Solenius, Xestocrabro 6-maculatus, texanus; Oxybel.: Notoglossa americana, frontalis, Oxybelus packardii; Philanth.: Cerceris clypeata, compar; Sphec.: Chalybion, Sceliphron; Pompil.: Lophopompilus philadelphicus, Planiceps niger, Poecilopompilus navus, Psammochares tenebrosus; Vesp.: Polistes variatus; Eumen.: Eumenes fraternus, Leionotus foraminatus, ziziae; Thynn.: Methoca; Chrysid.: Hedychrum violaceum, wiltii, Holopyga, Tetrachrysis eaerulans; Figit.: Psilodora impatiens, Xyalosema; Chalcid.: Chalcis, Perilampus cyaneus, fulvicornis; Ichneumon.: Cryptus exulans.

Diptera (59)-Chironom.: Ceratopogon fusculus fq, websteri; Mycetophil.: Sciara atrata; Bibion.: Scatopse pulicaria fq; Stratiomy.: Stratiomyia meigenii; Bombyli.: Argyramoeba albofasciata; Empid.: Empis distans ; Syrph.: Allograpta, Eristalis tenax, transversus, Mesogramma geminata, marginata fq, Orthoneura nitida, Paragus bicolor, tibialis, Sphaerophoria, Syritta ab, Syrphus americanus, ribesii; Conop.: Oncomyia, Zodion fulvifrons, nanellum; Tachin.: Belvosia unifasciata, Biomyia georgiae (Type Masicera sordicolor Tn.), Cistogaster occidua, pallasi, Euphorocera claripennis, Exorista confinis, Ocyptera tenuis, Pachyophthalmus signatus fq, Paradidyma, Spallanzania, Trichopoda pennipes, Viviania; Sarcophag.: Helicobia helicis fq, 4-setosa, Sarcophaga cimbicis, hunteri; Musc.: Graphomyia, Morellia fq, Myospila, Phormia regina, Pseudopyrellia; Anthomy.: Calythea, Limnophora fq, Phorbia acra, platura; Ortal.: Rivellia pallida, viridulans; Seps.: Nemopoda, Sepsis fq; Oscin.: Chlorops approximatinervis, mellea, proxima, Hippelates flavipes, plebejus, pusio, Oscinis coxendix, soror.

Coleoptera (7)-Hister.: Hister subrotundus; Lampyr.: Podabrus tomentosus; Cerambyc.: Euderces; Chrysomel.: Haltica; Mordell.: Mordella marginata fq, Mordellistena limbalis; Curculion.: Centrinites. 
Hemiptera (2)-Lygae.: Lygaeus; Caps.: Lygus.

Heracleum lanatum $(12: 459-60)$. - Blooms May 6i-June 17: 274 visitors observed, May 8-June 17; Pol, W.

Long-tongued Boes (7)-Meguhil.: Aluidumen simples \& 8 : Cerutin.: Ceratiua of $q$ se; $A$ p.: A pis se, fq : Nomed. : Henimumada f. Ilolonomada superba $d$, Nomada illinoensis ?. Phor 8; Rucer.: Tetralonia dileeta 우.

Short-tongued Bers (35)-Prosopid.: Prosopis illimoesuis of 8 .

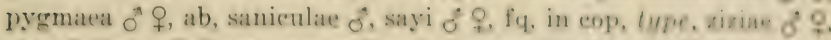

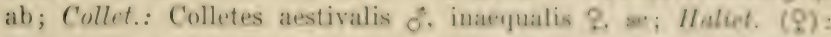
Agapostemon radiatus se, (hhloralietus pilosus so. fo, ngarous oc. versatus se, fq, Evylaeus peetoralis se, fq, Halietus lermusif, peral. lelus se, Oxystoglossa confusa, Seladonia; Sphecodini : Drepanium o, $\mathrm{fq}$, Machaeris illinoensis of, type, stygia + . Proteraner \&. Sphe-

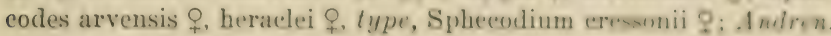
(q) : Andrena carlini, erythrogastra, pruni, sayi se. Opandrena miserahilis se, $\mathrm{fq}$, ressonii se, Parandrena, Ptilandrena gr. maculati. Trachandrena elaytoniae se, fq, erataegi, forbesii sc, hr.raclei so, type, nuda se, fq, rugosa se, spiraeana.

Other Hymenoptera (67)-Crabon.: Clytochrysus fạ. Lopbocrabro, Eetemnius, Hypocrabro, Protothyreopus rufifemur, Pseudocrabro $\mathrm{fq}$, Rhopalum pedicellatum, scufellatum, Sulenins fq, Testocrabro 6-maculatus, texamus; Orybul.: Notoglossa americana, Oxybelus niger, 4-notatus fq; Sphee: Chalybion. Chlorion, Seeliphron, Sphex vulgaris; Larr.: Notogonidea, Tachysphes aeutus; Pompil.: Anoplius illinoensis, virginiensis, Ceropales fraterna. Episyron. Planiceps feralis, Pompiloides marginatus, subviolaceus. Psammochares scelestus, tenebrosus; Vesp.: Polistes palligues fiq, variatus $\mathrm{fq}$, rubiginosus, Vespula germanica, maculata: Lumen. Ancistroeerus tigris, unifasciatus, Enmenes fraternus. Tcionotus anormis, clypeatus, foraminatus, illinoensis, megaera, exvophulariae, Symmorphus dehilis, walshianus; Tiphi: Tiphin elypeats. inornata, vulgaris ab, type; Chrysid.: Holopyesa: Rigit.: Tineoila, Euerilidia; Chalcid.: Inaltiehella ornata, Elasmus, Perilamgnus ful. vicornis; Evani.: Foenus tarsaturius; Iehneumon: Iobneunon flavizonatus, jueundus, fuuestus, longulus, Lampronota amerieans, Plyggadeuon vulgaris. Porizon eonotracheli, Trogus vulpinas, Try. phon affinis, Metopius terminalis; Bracon.: Mfierobracon: Tra thredin.: Dolerus aprilis.

Diptera (137)-Myctophil.: Seiara atrata fy, insuataus:

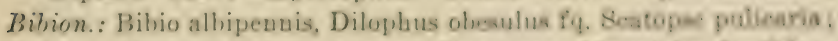
Taban. Chrysops indus, striatus ; Strutiomy.: Xemetelus fug Blan. 
tomyia cincta, Stratiomyia apicula, discalis, meigenii fq, quaternaria; Bombyli.: Anthrax alternata, Bombylius atriceps, Toxophora; Empid.: Empis distans fq, levieula fq, type, Pachymeria, Rhamphomyia fumosa, mutabilis $f q$, priapulus ab, rava, Tachydromia; Syrph.: Allograpta, Ceria, Chilosia petulea, Chrysogaster nigripes, Criorhina decora, umbratilis, Didea, Eristalis bastardi, dimidiatus $\mathrm{fq}$, transversus $\mathrm{fq}$, Helophilus similis, Mallota cimbiciformis fq, posticata, Mesogramma marginata, Myiolepta nigra, strigilata, Orthoneura nitida, pictipennis, Paragus bicolor, Pipiza femoralis, festiva, nigribarba, pulchella, Pterallastes, Sphaerophoria, Syritta, Syrphus americanus, ribesii, torvus, Temnostoma trifasciata type, Tropidia quadrata, Xylota bicolor; Conop.: Oncomyia, Zodion fulvifrons, nanellum; Tachin.: Alophora aeneoventris, fumosa, Archytas analis, aterrima, Belvosia unifasciata, Blepharipeza, Brachycoma sarcophagina type, Chaetogaedia analis, Cistogaster occidua, pallasi, Cryptomeigenia, Euphorocera claripennis, Gonia eapitata, Gymnochaeta, Gymnosoma, Hilarella, Hyalomyodes, Hyalurgus, Leucostoma atra, neomexicana, nigricornis, Pachyophthalmus signatus fq, Peleteria, Phorantha humeralis type, pruinosa type, purpurascens, robertsonii type, Polidea americana type, Senotainia trilineata $\mathrm{fq}$, Sturmia fraudulenta $\mathrm{fq}$, phyciodis, Tachina robusta, Trichopoda pennipes; Sarcophag.: Cynomyia, Helicobia helicis fq, 4-setosa, Sareophaga aldrichi, assidua, bullata, cimbicis, dux sarracenioides, impar, utilis; Musc.: Graphomyia fq, Lucilia caesar ab, sericata fq, sylvarum, Morellia fq, Phormia regina fq, Pseudopyrellia ab; Anthomy.: Anthomyiella $\mathrm{fq}$, Calythea, Limnophora, Phorbia acra fq, platura $f q$; Seps.: Sepsis; Oscin.: Chlorops mellea, proxima ab, Hippelates flavipes, plebejus, pusio $\mathrm{fq}$, Oscinis coxendix, trigramma, Siphonella cinerea, inquilina, Agromyz.: Agromyza latipes, Lobioptera.

Lepidoptera (7)-Nymphal.: Basilarchia archippus, Cercyonis, Charidryas, Phyciodes; Papilion.: Iphiclides, Laertias; Hesperi.: Polites.

Coleoptera (16)-Dermest.: Anthrenus castaneae, Cryptorhopalum haemorrhoidale, Orphilus; Lampyr.: Telephorus flavipes; Malach.: Anthocomus; Scarabae.: Euphoria sepulchralis, Trichius affinis, piger; Cerambyc.: Euderces; Chrysomel.: Diabrotica 12 punetata, vittata; Mordell.: Mordella marginata, Mordellistena biplagiata fq; Anthic.: Corphyra labiata; Melo.: Macrobasis; Curculion.: Centrinites..

Hemiptera (2)-Corimelaen.: Corimelaena pulicaria; Caps.: Phytocoris. 
Osmorrhiza longistylis $(12: 452,459-60),-B$ coms A Ant 21 May 23; 71 visitors observed, Mny 4-23; Mis, W.

Loug-tongued Bers (8)-Megachal,; Ceratomia 8. Dineratumuha

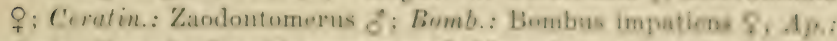
Apis: Nomad.: Gnathias cuneatus 9. Nomada parva of, myo 8

Short-tongued Bees (24)-Prosopid.: Prowopis illinonsis 6.

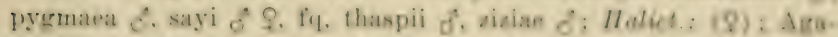
postemon radiatus se, splendens. Ausochlova viridula, Chloraliedus eaerulens, obseurus se, type, pilosus se, sparsus se, versatus se, Curtisapis coriacea se, furhesii se, Evylaens purtoralis so, ab, t maculatus se, ab. Haliefus brouxii. Uxysteglemen confons ab, gure. Seladonia: Spheeolini: Proteraner o: Andreas: Andrens povand q, sayi $q$.

Other Hymenoptera (4)-Eumen.: Aneistreerus tikcris. A.u menes fraternus; Chrysid.: Holochrysis hilaria; Evoni.: Foenus tarsatorius.

Diptera (27)-Bumbyli: Alihiehia, Bombylius major: Jimpud: Empis distans ab, humilis type. loripedis: Surph.: Allograpes. Chilesia eyanescens ffy. petulea, Mesugramma geminata, murcinats. Paragus hieolor, tibialis, Pipiza Pemoralis, Rhingia, Spluswoplonea, Sphegina campanulata type, rufiventris, Syritta ab, Syrphus ribesii; Compp.: Myepa vesieulosa ; Tarhin.: Gymnosmua, Peleteria. Siphona fil: Anthomy.: Ilylemyia inornata, Phorbia platura: Pall.; Chyliza; Oscin.: Siphonella oseinina.

Lepiloptera (2)-P'apilion.: Eurymus; Hesperi: Thasus martialis.

Coleoptera (5)-Bruch.: Bruchus minus: Mordell.: Mordel. listena biplagiata; Anthic.: Corplayra torminalis: Curculuan: Idiostethus subealvus, tubulatus.

Hemiptera (1)-Caps.: Lygus.

Oxypolis rigidior $(12: 459-60)$.-Blons July 31-8eptentes $8 ; 157$ visitors observed, Augrust $14-$ September $8 ;$ Pol, W.

Long-tongued Bees (1)-Ap.: Apis.

Short-toneura Beos (6)-Prosogril (ó 9 ) : Prompis pyomaen. ziziae: Hulict.: Chloralietus sparsus \&, se. reratus of \$, nc. Ifallecus lerouxii ; Sphecodini: Sphecodes arvensis of.

Other Hymenoptera (73)-Crubrun.: Lindesilus omsus, Bol. enius, Xintorabo texanus, trifusoiarun: Orybet: Xotaglems

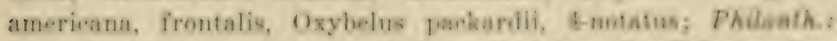

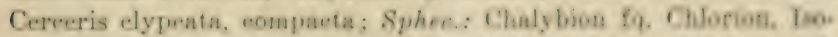
dontia apicalis. Priononyx atrata, Sceliphron; Lorr: Asians unh

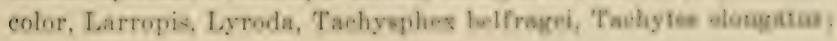


Bembic.: Stizus; Nysson.: Hoplisus simillimus, Pseudoplisus phaleratus; Pompil.: Arachnoproctonus, Cryptocheilus fulgifrons, Lophopompilus philadelphicus, Priocnemoides fulvicornis, Psammochares relativus, Sophropompilus; Vesp.: Polistes variatus, rubiginosus; Eumen.: Leionotus foraminatus, fundatus, histrionalis type, ziziae; Scoli.: Elis 5-cincta, Scolia bicincta; Tiphi.: Tiphia clypeata, punctata type, transversa; Myrmos.: Myrmosa unicolor; Mutill. (ơ): Sphaerophthalma, Mutilla hexagona; Chrysid.: Hedychrum violaceum, Holopyga, Tetrachrysis caerulans, venusta; Figit.: Aspicera, Cothonaspis, Figites impatiens fq, Xyalosema; Chalcid.: Eutrichosoma, Bruchophagus, Leucopsis, Perilampus fulvicornis, hyalinus; Ichneumon.: Ichneumon flavizonatus, Ceratosoma ab; Bracon.: Microdus simillimus, Agathis areolata, Chelonus, Urosigalphus, Apanteles crassicornis, Toxoneuron abdominale, Macrodyctium, Microplitis croceipes, gortynae, Monogonogastra, Iphiaulax, Opius fuscipennis.

Diptera (53)-Mycetophil.: Sciara atrata; Empid.: Parempis; Syrph.: Allograpta, Mesogramma geminata, marginata, polita, Paragus bicolor, Spilomyia longicornis, Syritta, Syrphus ribesii; Pipuncul.: Chalarus; Conop.: Conops brachyrrhynchus, Zodion nanellum; Tachin.: Alophora aeneoventris, Belvosia unifasciata, Cistogaster occidua, Clytiomyia, Epigrimyia geniculata type, Exorista confinis, pyste, Euphorocera claripennis, Gymnosoma, Hypostena indecisa type, Ocyptera dosiades, Pachyophthalmus signatus, Phorantha pupurascens, Senotainia rubriventris, trilineata, Spallanzania, Trichopoda pennipes; Sarcophag.: Helicobia helicis, 4-setosa, Sarcophaga assidua, cimbicis; Musc.: Lucilia sericata, sylvarum, Morellia, Musca, Pseudopyrellia; Anthomy.: Anthomyiella, Limnophora, Lispa consanguinea, Phorbia platura; Trypet.: Euaresta bella; Ephydr.: Ochthera; Oscin.: Hippelates flavipes $\mathrm{fq}$, plebejus, pusio, Oscinis coxendix, trigramma, Siphonella cinerea; Agromyz.: Agromyza latipes, Leucopis.

Lepidoptera (3)-Nymphal.: Basilarchia archippus; Lycaen.: Eupsyche; Hesperi.: Limochores taumas.

Coleoptera (16)-Coccinell.: Coccinella sanguinea, Hippodamea convergens; Latridi.: Corticaria ; Lampyr.: Chauliognathus pennsylvanicus; Scarabae.: Euphoria sepulchralis; Chrysomel.: Diabrotica atripennis, 12-punctata, Luperaltica; Bruch.: Bruchus cruentatus; Mordell.: Mordella melaena, Mordellistena limbalis; Melo.: Epicauta cinerea, pennsylvanica, Pyrota germari; Rhipiphor.: Rhipiphorus limbatus; Curculion.: Centrinus picumnus.

Hemiptera (4)-Lygae.: Oncopeltus; Caps.: Lygus, Plagiognathus; Acanthi.: Triphleps. 
Neuroptera (1)-Chrysop.: Chrysopa.

Aug. 20, 1909, insects taken as they cane showed the following 30 species and 85 individuals:

Short-tongued Bees $(1: 3)$ - Dulict: Chloralietus versutue 3.

Other Hymenoptera (15:30)-Crabrun.: Sulenins 1, Xeata. erabro texanus 3 ; Orybel.: Notoglosa frontalis 1 ; Aphec. Chaly. bion 15, Priononyx atrata 1, Sueliphrou 4; Larr.: Iyroda 1: Nus. son.: IIoplisus simillimus 1. Pseudoplisus phateratus 2; Pempal: Lophopompilus philadelphiens 2. P'rionemoides fulviooros \&; Vesp.: Polistes rubiginosus 1, variatus 2; Tiphi.: Tiphia elypoata 1; Chrysid. 'Tetrachrysis caerulans 1.

Diptera (14:44) - Syrph.: Spilomyia longicornis 1, Syritta 5, Syrphus ribesii 1; Techin.: Alephora aeneoventris 1, Relvosia uni. fasciata 9, Exoristal pyste 1, Oeyptera dosiades 1, Pachyophthalume signatus 4, Spallanzania 2; Sarcophag.: Helicobia helieis 7 , Sarcophaga assidua 4, eimbieis 5; Muse.: Morrllia 2; Anthomy.: An. thomyiella 1.

Pastinaca sativa (int, $12: 459-60)$. - Bhoms May 1.1-Auruat 10 ; 298 visitors observed, June 2-July 9; Pol, Y.

Long-tongued Bees (7)-Megurhil.: Megarhile potulaus $9:$ Ceratin.: Ceratina . . se; Bomb.: Bombias separatus $\forall$, Bombus amerieanorum $\&$ \&. impatiens 9 ; Ap.: Apis; Anthophor: Andiemoessa $\sigma^{7}$.

Short-tongued Bees (2.) -Prosopid.: Prosopis illinoensis of ?. type, pygmaea of sayi $\delta$, ziziae of $q$; Collet.: Colletes hreviconis

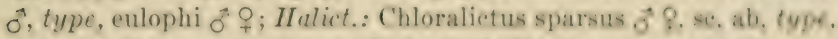
versatus of f. se, Curtisapis forbesii of. Evylaens areuatus of pee. toralis $q$, se, Halietus parallelus f. Oxystoglosan confusa o. Sola. donia of; Spheeodini: Sphecodes arvensis of ? ab. clematidie of. Sphecodinm eressonii of $\$$; Andren.: Andrena platyparia \&, promi

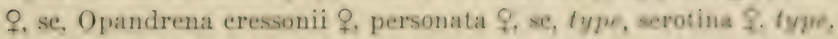
Trachandrena erataegi $q$, hippotes $q$, nuda $q$, sc.

Other Ilymenoptera (94)-Crabron.: Clylochrysus, Retemnins, Hypoerabro, Protothyreopus rufifemur, Psondimerabo, Solenius fy. Xestoerabro 6-maculatus, trifasciatus; Oxybel.: Notogloens amer6. cana, enarginata, frontalis type. inornata, Oxyiolus 4.miatos; Trypoxyl.: Trypoxylon elavatum; Philanth.: Crvervin clgyreta, comparta, fumipmnis, kennicottii, Philanthus punctatue: Sphe: Ammobia ichneumonea. pennsylvaniea, Chalybion. Imdentia apicalis, Seeliphrou, Sphes uigrieans, vulgaris; Lare. Avtatus unieolor. Lyroda; Nysson.: Pseudoplisus phaleratus: Pompil.: Anoplles ill.

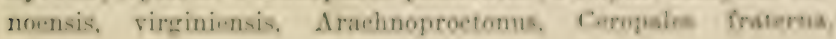


Lophopompilus atrox, philadelphicus, Poecilopompilus navus, Pompiloides americanus fq, divisus, marginatus, subviolaceus, Psammochares tenebrosus; Vesp.: Polistes pallipes, Vespula maculata; Eumen.: Ancistrocerus campestris, tigris, unifasciatus, Eumenes fraternus, Leionotus foraminatus fq, fulvipes, scrophulariae, ziziae, Monohia, Symmorphus debilis, walshianus, Zethus; Scoli.: Elis 5cincta; Tiphi.: Tiphia vulgaris; Mutill. (ठ): Sphaerophthalma, Mutilla hexagona; Chrysid.: Hedychrum violaceum, Holopyga fq, Tetrachrysis nortonii; Figit.: Aspicera, Psilodora impatiens; Chalcid.: Leucospis, Perilampus triangularis, cyaneus, platygaster, Pachyneuron ; Evani.: Foenus incertus, tarsatorius, Hyptia; Ichneumon.: Agrothereutes limatus, Ichneumon flavizonatus, jucundus, succinctus, Colpognathus, Cryptus rufoannulatus, Eiphosoma, Glypta simplicipes, Hemiteles, Porizon conotracheli, Proclitus, Trogus, Metopius terminalis; Bracon.: Orgilus, Euphorus; Cardiochiles, Habrobracon, Microplitis gortynae, Iphiaulax; Tenthredin.: Hylotoma humeralis ; Scelion.: Teleas.

Diptera (114)-Mycetophil.: Sciara atrata, vulgaris; Bibion.: Scatopse pulicaria; Taban.: Chrysops striatus, Therioplectes; Stratiomy.: Allognosta fuscitarsis, Stratiomyia meigenii; Bombyli.: Anthrax sinuosa, Argyramoeba oedipus; Scenopin.: Scenopinus; Empid.: Empis distans, Parempis; Syrph.: Allograpta fq, Baccha aurinota, fuscipennis, Didea, Eristalis aeneus, dimidiatus, flavipes, transversus, Helophilus latifrons, similis, Mallota cimbiciformis, Mesogramma geminata, marginata fq, Orthoneura nitida in cop, Paragus bicolor, tibialis, Pipiza festiva, nigribarba, pulchella, Platychirus hyperboreus, quadratus, Sphaerophoria, Syritta fq, Syrphus arcuatus, ribesii, Tropidia quadrata; Conop.: Conops brachyrrhynchus, Myopa vesiculosa, Zodion nanellum; Tachin.: Araba, Belvosia unifasciata fq, Biomyia, Blepharipeza, Brachycoma intermedia type, Ceratomyiella, Chaetoplagia, Cistogaster occidua, Clytiomyia, Cryptomeigenia, Distichona varia, Ennyomma globosa, Eumyotheria illinoensis type, Euphorocera claripennis, Exorista confinis fq, nigripalpis, Frontina aletiae, frenchii, Gymnosoma, Gymnoprosopa clarifrons type, Linnaemyia fq, Macquartia, Ocyptera tenuis, Pachyopthalmus signatus, floridensis, Phorantha purpurascens, Senotainia rubriventris, trilineata, Spallanzania, Sturmia inquinata, Tachina mella, rustica, Trichopoda pennipes fq, Trichophora, Winthemia fq, Xanthomelana; Sarcophag.: Helicobia helicis fq, 4-setosa, Sarcophaga alcedo, assidua, cimbicis, haemorrhoidalis, hunteri, sinuata; Musc.: Calliphora erythrocephala, Compsomyia fq, Graphomyia, Lucilia caesar, Morellia fq, Musca, Myospila, Phormia regina, Protocalliphora, Pseudopyrellia; Anthomy.: Calythea, 
Fyetolosia, Limnophora, Ophyra fin, Phorbia arre fiq Orful: Camptoneura, Rivellia viridulans, Seoptera; Sagramys: Sapromyza vulgaris; Seps.: Sepsis; Oscin.: Chlorops asomilin, uelles. proxima, Hippelates flavipes, pusio, Mrromyza, Owinis noror , Ayromyz.: Lobioptera.

Lepidoptera (9) -Nymphal.: Anosia, Basilarehia antyanas. Hypatus; Lycarn.: Chrysophanus, Cyaniris, Theola ealanus, He. peri: Limochores taumas; Sesi.: Siesia pietipos; Pyromorph : Mar risina.

Coleoptera (42)-Ciecrinell.: Coecinella 9-notata, sanguines. Hippodamea parenthesis, convergens, Megilla ; Dermest.; Anthrans castaneate, Attagenus fq. Cryptorhopalum haemorthoidale; Nifulul: Carpophilus; Elater.: Agriotes; Buprest.: Agrilus diffirilis, coms: Lumpyr.: Calopteron, Chaulingnathus marginatus, Pliotinus, I'oda. brus rugulesus, tomentosus. Pyractomena, Telephorus dlavipes: Maluch.: Anthoemus, Attalus; Scarebae.: Euphoria fulgida, smul. ehralis, Trichius piger; Cerambye.: Aemanops direeta, Callenusys fuscipennis, sanguinicollis, Euderees. Typocerus sinuatus, velutinos; Chrysomel.: Anomoea, Diabrotiea vittata, Pachybrachys, Tri. rhabda: Milinelry.: Noihus; Mordell.: Mordella marginsta. melaena, s-punctati, Mordellistena ormata, Pentaria: Melo: Epi. eauta cinerea, Maerobasis.

Itemiptera (6)-Corimeleen.: Corimelaena puliearia fy : Beryt.: Corizus: Lygue: Lyqaeus: Cups.: Lygus. Phytuouris, Placrogna. thus.

Neuroptera (1)-Chrysop.: Chrysopa.

Polytaenia nuttallii $(12: 459-60)$.- Blooms May :-June 17; 7h visitors observed, May 9-June 8; Mis, Y.

Long-tongued Bees (4)-Megurhil: Coelioxys s-dentata of. Bumb.: Dombus americanorum $9:$ Ap. Apis: Nomed.: Nomada illinoensis .

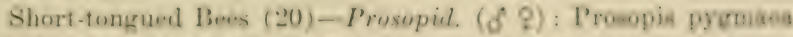

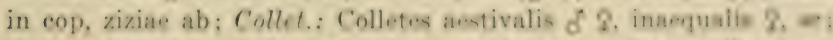
IIalict (2): Augnehlora fervida, viridula, Chloralietus jilieun m. fruinusus se, sparsus se. tegularis se, versatus so, Evylaeno peetoralis. Odontalictus se, Oxystoglosat similis se, SolaAnnin s.; An.

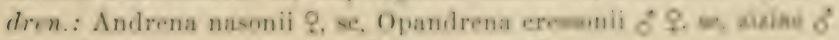
$\uparrow$, se, Trachandrena crataegi $ᄋ$, se, forbesii $q$.

Other Hymenoptera (20)-Crobron,: Ectemnims, Hypueralim, Pseudoerabro, Solenius; Sphee.: Seeliphren; Verp.: Polist-s pallijes, variafus; Eumen.: Ancistrowerus eaupestris, Eumenes froternus, Leionotus anormis, elypeatuk, foraminatus, bisiramalic. 
illinoensis, scrophulariae, vagus, ziziae, Symmorphus walshianus; Tiphi.: Tiphia inornata; Chalcid.: Eurytoma.

Diptera (17)—Strotiomy.: Allognosta obscuriventris, Nemotelus, Stratiomyia apicula; Syrph.: Allograpta, Mesogramma geminata, marginata, Paragus bicolor, tibialis, Sphaerophoria, Tropidia mamillata; Conop.: Oncomyia, Zodion fulvifrons; Tachin.: Cistogaster occidua, pallasi, Leucostoma atra, nigricornis, Linnaemyia, Phorantha purpurascens; Musc.: Pseudopyrellia; Anthomy.: Phorbia acra ab, platura; Ortal.: Rivellia pallida; Oscin.: Chlorops proxima, Hippelates flavipes; Agromyz.: Leucopis, Lobioptera.

Coleoptera (1)-Curculion.: Centrinites.

Hemiptera (3)-Beryt.: Corizus; Pentatom.: Euschistus ictericus; Caps. : Lygus.

May 20, 25, 26, 1910, insects taken as they came to the flowers showed the following 37 species and 294 individuals:

Long-tongued Bees (2:5)-Bomb.: Bombus americanorum 1; Ap. Apis 4.

Short-tongued Bees $(12: 126)$-Prosopid.: Prosopis ziziae 3 ; Collet.: Colletes aestivalis 18; Halict.: Augochlora fervida 4, viridula 2, Chloralictus versatus 18, Evylaeus pectoralis 1, Oxystoglossa similis 9, Seladonia 5; Andren.: Opandrena cressonii 9, ziziae 45, Trachandrena crataegi 11, forbesii 1 .

Other Hymenoptera $(9: 32)$-Crabron.: Hypocrabro 1, Solenius 2; Vesp.: Polistes variatus 15; Eumen.: Eumenes fraternus 1, Leionotus foraminatus 4, histrionalis 4, illinoensis 1, ziziae 2, Symmorphus walshianus 2 .

Diptera (12:127)—Stratiomy.: Nemotelus 3, Stratiomyia apicula 2; Syrph.: Mesogramma geminata 1, marginata 11, Paragus bicolor 1, tibialis 1; Conop.: Oncomyia 1, Zodion fulvifrons 1; Musc.: Pseudopyrellia 2; Anthomy.: Phorbia acra 99, platura 4; Agromyz.: Lobioptera 1.

Hemiptera (2:4)-Beryt. Corizus 3; Pentatom.: Euschistus ictericus 1.

Sanicula canadensis.-Blooms May 30-July 20; four visitors observed, June 15, 17; Mis, W.

Short-tongued Bees $(3: 3)$-Halict. ( $(q)$ : Chloralictus versatus 1, Evylaeus 4-maculatus 1; Prosopid.: Prosopis saniculae $q, 1$.

Diptera (1:2)—Syrph.: Mesogramma geminata 2.

Sanicula marilandica.-Blooms May 6-June 5; 31 visitors observed, May 14, 16, 25 ; Mis, Y.

Short-tongued Bees (14)-Halict. ( $($ ) : Augochlora viridula, Chloralictus sparsus, tegularis, Curtisapis coriacea, Evylaeus foxii, 
Oxystoglossa confusa; Prosopul.: I'rosopis ryormava 6 . eaniumlae

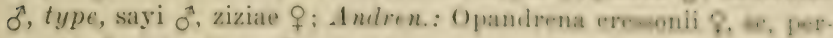
sonata $\sigma^{*}$, ziziae $q$, se, Trachandrena rugosa $q$, se.

Other Irmenoptera (3)-Eumen.: Aneistrocorma tipris sym. morphus debilis, Bracon.: Braeon ashmeadi type.

Diptera (12)-Mycetophil.: Sciara atrata: Rinpil.: Ibruchy. stoma robertsonii type, Empis distans, nula ; Syrph.: Meroramma geminata, marginata; Tachin.: Siphoma; Muse.: Myospila; An. thomy.: Inomalomyia prostrata; Supromyz.: Saprumyzu Lunpipennis; Heteroneur.: Clusia; Oscin.: Siphonella oscinina.

Hemiptera (2)-Corimcluen.: Corimularna pulicaria; Coms.: Lopidea.

Sium cicutaefolium $(12: 459-60)$.-Blooms July 19 Siptem. ber 7 ; 220 visitors olserved, July 20-September 1: Pul, W.

Long-tongued Bees (4)-Bomb. (8. c) : Bombus americanomum. impatiens; Ap.: Apis; Eucer.: Melissodes agilis $\delta^{\star}$.

Short-tongued Pees (17)-Prosopid.: Prosopis pyguma of sayi o, ziziae $q$; Collet.: Colletes americamus of, eulophi $\delta$ : Ilatiet.: Chloralietus illinoensis $\hat{\delta} \mathcal{q}$. sparsus $q$, versatus of $\mathrm{R}$, se, zeplyyrus $\widehat{\sigma}$ ․ Curtisapis coriacea ô $q$, Evylaeus arruatus ó, trumeatus ?. Halietus lerouxii ơ, Odontalictus $q$, Seladonia $q$; Spheeodini : Machaeris stygia $\delta \rightarrow$ fq, type, Sphecodes arvensis $\delta$.

Other IIymenoptera (97)-Orabron.: Clytochrysus fy, E.temnius fq, Lindenius errans $\mathrm{fq}$, Solenius, Xestoerahro 6-maeulatus, trifasciatus; Oxybel.: Notoglossa americana fq, emarginata, frontalis $\mathrm{fq}$, Oxybelus niger type, packardii, 4-notatus fy: Tryporyl.: Trypoxylon clavatum; Philanth.: Cereeris hicornuta, elypata, ceho. finitima, kennieottii, prominens, Philanthus punetatus; Spher: Chalybion, Chlorion, Isodontia apiealis, Priononyx thomae, Sceliphron, Sphex gracilis, vulgaris; Larr.: Larropsis, Lyroda, Noto. gonidea f(1, Tachysphex terminatus, Tachytes elongatus; Riembir: : Megastizus; Nysson.: Hoplisoides, Nysson plagiatus, Zanywoon plesia, Pseudoplisus phaleratus; Pompil.: Ageniella aroepta, longula, Ceropales fraterna, Episyron, Lophopumpilus atrus, phal. adelphicus, Pompiloides marginatus, subviolaceus. Psammombars relativus, tenebrosus; Vesp.: Polistes pallipes, variatus, Vespula germanica; Eumen.: Ancistrocerus eampestris, tigris, Eumeus fraternus, Leionotus foraminatus, serophulariae, zizian. Monobis: Scoli.: Elis interrupta, 5-cincta, Scolia bicineta; Tiphi: Tighia punctata; Bethyl.: Goniozus; Thynn.: Mefluesa ; Myrmus.: Myruoes parvula type; Mutill.: Sphaerophthalma of Chrusid.: Holechryols verticalis, Holopyga, Tetrachrysis earrulans; Figit.: Euenils mel. 
lipes fq, Eucoilidia, Figites impatiens, Psilodora erythropus, impatiens, Trirhoptrasema, Xyalosema ; Chalcid.: Chalcis, Haltichella ornata, xanticles, Smiera debilis; Elasmus, Bruchophagus, Eurytoma, Leucospis, Perilampus cyaneus, Eutelus, Syntomopus; Ichneumon.: Agrothereutes mundus, Ichneumon flavizonatus, Cremastus retiniae, Cryptus persimilis, Limneria eurycreontis; Bracon.: Chelonus; Apanteles illinoensis, terminalis, Microplitis ceratomiae, Iphiaulax.

Diptera (74)—Mycetophil.: Sciara atrata; Taban.: Chrysops striatus; Stratiomy.: Stratiomyia meigenii; Bombyli.: Anthrax alternata, Exoprosopa fasciata, fascipennis, Sparnopolius, Toxophora; Empid.: Parempis; Syrph.: Allograpta, Eristalis aeneus, tenax, Mesogramma geminata, marginata, polita, Orthoneura nitida, Paragus tibialis, Pipiza pulchella, Platychirus quadratus, Syritta, Syrphus ribesii, Tropidia albistylum, quadrata; Tachin.: Belvosia unifasciata, Chaetogaedia analis, Clytiomyia, Distichona varia, Ennyomma globosa, Euphorocera claripennis, Frontina aletiae, Gymnosoma, Hypostena variabilis, Leucostoma atra, Linnaemyia, Pachyophthalmus signatus, floridensis, Panzeria fq, Phasiociista, Phorantha purpurascens $\mathrm{fq}$, Senotainia rubriventris, trilineata $\mathrm{fq}$, Spallanzania, Sturmia distincta, Trichopoda pennipes; Sarcophag.: Helicobia helicis, 4-setosa, Sarcophaga assidua fq, haemorrhoidalis; Musc.: Compsomyia, Graphomyia, Lucilia sericata, Morellia, Musca, Phormia regina, Protocalliphora, Pseudopyrellia, Stomoxyz; Anthomy.: Calythea, Coenosia fuscopunctata, Homalomyia prostrata, Limnophora, Ophyra, Phorbia acra; Ortal.: Euxesta; Trypet.: Plagiotoma, Seps.: Themira; Ephydr.: Ochthera, predaceous; Oscin.: Chlorops proxima, Hippelates flavipes, pusio, Meromyza, Siphonella cinerea, Agromyz.: Agromyza latipes.

Lepidoptera (5)-Nymphal.: Anosia, Hypatus; Lycaen.: Cyaniris, Eupsyche; Papilion.: Pieris.

Coleoptera (1)-Coccinell.: Coceinella sanguinea, Megilla; Dermest.: Attagenus; Lampyr.: Chauliognathus pennsylvanicus, Ditemnus, Photinus; Malach.: Collops; Scarabae.: Euphoria sepulchralis; Chrysomel.: Diabrotica 12-punctata, longicornis, vittata; Bruch.: Bruchus discoideus, hibisci, obtectus; Mordell.: Mordella marginata, melaena, 8-punctata; Melo.: Epicauta pennsylvanica; Curculion.: Centrinus picumnus.

Hemiptera (4)-Corimelaen.: Corimelaena pulicaria; Lygae.: Lygaeus; Caps.: Lopidea fq; Acanthi.: Triphleps.

Taenidia integerrima (12:459-60).-Blooms April 27-June 14 ; 113 visitors observed, May 10-June 3 ; Mis, Y. 
Long-tongued Bies (14)-Meguchil: Meliames simples 8.

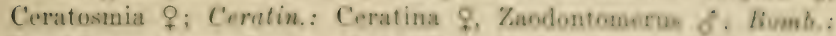
Bombias separatus \&; $A$ p.: Apis; Nomad. Centrias americanue of \&. Gnathias cuneatus ․ ovatus \&. Holonomala affabilis P. Numseds

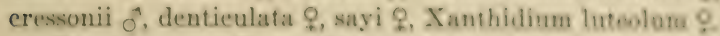

Short-tongued Bees (33)-Prosupil.s Prompris illinaenais 28.8 .

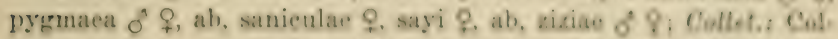
letes aestivalis of; Halict. (9): Augoehlora Pervidh, Dhloralletua nymphaearum, sparsus se, fq, tegularis, versatus $=$, zephyros, Evylaeus foxii se, peetoralis, 4-maculatus se. Odentalietus fo. Ory. stoglossa confusa, pura, similis se, ab, Seladonin fog, Sphocodini: Machaeris stygia \&. Proteraner of, Sphecodes arvensis \&. Spliesodium pirupiuellae o, type; Andren.: Andrena nasonii $\delta$ \&. so, ab, Opan-

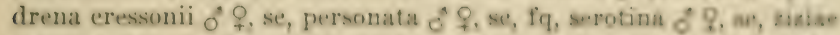

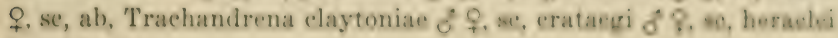
ㅇ, nuda + .

Other Hymenoptera (21)-Crubron: Clytochrysus, Solenius, Xestoerabro trifasciatus; Sphec.: Chalybion, Sceliphron. Spbex vul. garis; Pompil.: Anoplius illinoensis; Eumm. : A beistrocerus theris, Eumenes fraternus, Leionotus anormis, clypeatus, foraminatus, his. trionalis, illinoensis, serophularine, Symmorphus debilis; Tiphi: Tiphia elypeata; Chrysid.: Chrysis intrieata; Eveni.: Fenus in. certus; Ichneumon.: Angitia, Pharsalia.

Diptera (34)-Stratiomy.: Nemotelus; Bombyli.: Anthras sinn. osa f, Toxophora; Empid.: Empis levieula, Parempis; Byrgh.: Baccha fuscipennis, Chilosia petulea, Mesogramma geminata, marginata, Orthoneura nitida, Sphaerophoria, Syritta fol, Syrphus amerieanus, ribesii ; Conop.: Oneomyia, Zodion fulvifrons, Tuchis.: Cistogaster oecidua, Leueostoma nigrieornis, Limnarmyin, Deyptera lata, tenuis, Peleteria, Trichophora; Sarcophagy.: Malleabis helieis, Muse.: Lueilia serieata, sylvarum : Anthumy.: Coenoeis antiea : Ortul.: Rivellia pallida: Sapromyr.: Sapromyen volearlo;

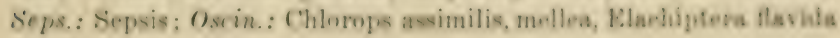
$\mathrm{fq}$, Hippelates plebejus.

Lepidoptera (1)-Nymphal.: Charidryas.

Coleoptera (9)-Mister.: Hister subrotunilus; Meluah : Antbo. eomus: Chrysomil.: Bahia; Bruch.: Bruehus bivolnerates bibseci. Mordell. Mordella marginata, Mordellintena aperas; Curculum. Centrinites, Limnobaris.

Hemiptera (1)-Corimelaen.: Corimelaena lateralis.

Thaspium aureum atropurpurem $(12: 444)$ - (Heums May 9July 1; one visitor observed, June 9; Mis, R.

Diptera.-Empid.: Empis loripedis. 
Thaspium aureum trifoliatum (12:459-60).-Blooms May 13July 18; 60 visitors observed, June 5-17; Mis, Y.

Long-tongued Bees (4)-Megachil.: Trypetini: Neotrypetes truncatus $\sigma^{\lambda}$, Coelioxyini : Coelioxys sayi $\sigma^{\lambda}$; Nomad.: Nomada denticulata + , sayi $q$.

Short-tongued Bees (21)-Prosopid.: Prosopis crataegi $\delta^{\lambda}$, illi-

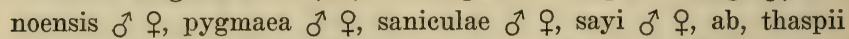
+ , type, ziziae $\delta^{\lambda}+$, fq; Collet.: Colletes eulophi $\delta^{\lambda}$; Halict. (q): Augochlora viridula se, Chloralictus sparsus se, tegularis se, Evylaeus pectoralis se, Halictus lerouxii, Oxystoglossa pura, similis se; Sphecodini : Proteraner $\delta^{\lambda}$; Andren.: Andrena earlini + , Opandrena cressonii + , se, personata $\hat{\delta}$, ziziae + , sc, ab; Panurg.: Heterosarus ㅇ.

Other Hymenoptera (10)-Crabron.: Pseudocrabro, Solenius; Sphec.: Sphex vulgaris; Eumen.: Ancistrocerus unifasciatus, Eumenes fraternus, Leionotus foraminatus, fulvipes, scrophulariae, Zendalia acolhuus; Evani.: Foenus tarsatorius.

Diptera (20)-Empid.: Empis loripedis; Syrph.: Allograpta, Baccha fuscipennis, Mesogramma geminata, marginata, Paragus bicolor, tibialis, Sphaerophoria, Syritta, Volucella vesiculosa, Xanthogramma flavipes; Conop.: Oncomyia, Zodion nanellum; Tachin.: Cistogaster occidua, Exorista confinis, Leskiomima prima, Siphona; Sarcophag.: Helicobia helicis; Musc.: Phormia regina; Seps.: Sepsis.

Lepidoptera (1)-Lycaen.: Cyaniris.

Coleoptera (2)-Cerambyc.: Euderces; Bruch.: Bruchus cruentatus.

Hemiptera (2)-Lygae.: Lygaeus; Caps.: Lopidea.

Zizia aurea (12:451-2, 459-60).-Blooms April 18-June 9; 179 visitors observed, May 3-26; Mis, Y.

Long-tongued Bees.(19)-Stelid.: Microstelis $\widehat{\jmath}$, Megachil.: Alcidamea simplex $\sigma^{\top}+$, Osmia atriventris $q$, se, pumila $q$; Ceratin.: Ceratina $0^{\lambda} q, \mathrm{ab} ;$ Bomb. ( $\left.q\right)$ : Bombias auricomus, scutellaris, separatus, Bombus americanorum, consimilis, impatiens; Ap.: Apis se ; Nomad.: Centrias americanus $\delta$, Gnathias ovatus $q$, Holonomada affabilis $q$, superba $\delta^{\top}+$, Nomada denticulata $\delta^{\lambda}+$, sayi $\delta^{\lambda}+$; Eucer.: Tetralonia dilecta $\delta^{\wedge}$..

Short-tongued Bees (42)-Prosopid.: ( $\left.\sigma^{1} \uparrow\right)$; Prosopis pygmaea in cop, sayi, ziziae fq, type; Collet.: Colletes aestivalis $\sigma^{\top}+$, ab, inaequalis $q$; Halict. ( $q)$ : Agapostemon radiatus, Augochlora fervida, viridula, Chloralictus albipennis se, type, coreopsis, pilosus sc, fq, pruinosus se, sparsus sc, fq, tegularis sc, type, versatus sc, ab, 
zephyrus, Curtisapis coriaces, forbesii, Evglaeus foxis, p-mandis se, 4-maculatus, Halietus lerouxii fq, parallelus, Odontulictur $=$, fo Oxystoglossa confusa se, fiq, pura, similis se, ab, Pavaliens wloty. parius type, simplex, Seladonia se, ab: Sphecodini: Protevauser of?

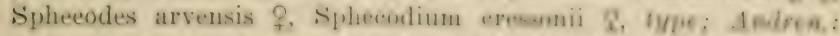
Andrena illinoensis \&, nasonii $\delta$, sayi $\&$, Opaudreus exemuil $\delta$ \&.

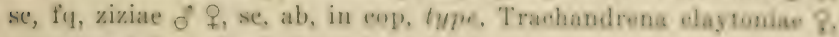
crataegi $\sigma^{*} q$, se, fq, forbesii $q$, nuda $q$.

Other Iymenoptera (32)-Crabron: Clytirchrysns, E temuim $\mathrm{f}_{\mathrm{q}}$. Pseudoerabro, Solenius ab, Xestocrabro 6-maculatus, tessuos, trifasciatus fy; Osybel.: Oxybelus 4-notatus; Pewpel: Anoptlos illinoensis, Episyron, Psammochares tenebrosus; Vespi.: Probians variatus; Eumen.: Ancistrocerus eampestris, tieris. Eumenes fraternus, Leionotus anormis $\mathrm{fq}$, elypeatus fq, forminatus his trionalis, illinoensis, megaera, perennis, serophulurian, vagus, siaine ab, Symmorphus walshianus: Tiphi.: Tiphia clypeata; Chrysid.: Gonochrysis; Figit.: Eucoilidia; Ichneumon.: Cremastus magogos. Exyston ab; Tenthredin.: Pseudosiobla.

Diptera (65)-Mycelophil.: Sciara atrata; Bibien.: Bibio alb. pennis; Simuli.: Simulium; Stratiomy.: Allognosta oho-uriventris. Nemotelus; Bombyli.: Aldriehia; Empid.: Empis distans, Lovijedie; Syrph.: Chilosia petulea, Chrysogaster ontario, Eristalis dimidiatus. latifrons, Helophilus latifrons, similis, Mecogramma marginata. Orthoneura pietipennis, Paragus biecolor, tibialis, Sphaerughuria. Sphegina rufiventris, Syritta, Syrphus ribesii. Tropidia mamilleta; Conop.: Oncomyia, Zodion fulvifrons; Tuchin.: Alophora arsomwn. tris, Apinops, Ceratomyiella enuiea type, Cistugaster awidun ab, pallasi, Leueostuma atra tyye, Limnemyia, Oeyptera lata. Paehy. ophthalmus signatus, Phorantha magna, purpurnsoms, Sipbonas, Sturmia nigrita type, Tachina rustica, Trichognols peuniyes: Sarcophag.: Helicobia helicis, Sareophaga siuuata; Mune: Lueliha caesar, sylvarum, Morellia, Phormia regina, Psocdopyrellis: Anthomy.: Calythea, Cnenosia lacteipennis, Hylemyis insmusta ab, Phorbia acra ah, cinerella, platura; Orful.: Rivellia jallids; Sopu: Sepsis; Oscin.: Chlorops arsimilis, proxime, palveres, Hipnelates flavipes, plebejus, Meromyza, Oscinis sorur, Sighumella einems: Agromyz.: Agromyza aeneoventris.

Lepidoptera (5)-Nymphal.: Phyciodes: Lycaen.: Rivern. Heodes: Papilion. : Eurymus: Nochu.: Plusia simples.

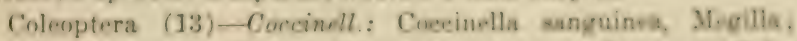
Erotyl.: Languria: Hister.: Hister americenus: Lampyr.: Tele-

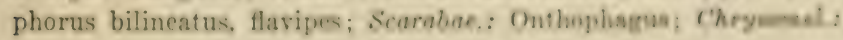


Ceratoma, Diabrotica 12-punctata, vittata ; Curculion.: Centrinites, Centrinus picumnus; Cerambyc.: Acmaeops nigripennis.

Hemiptera (3)-Pentatom.: Euschistus ictericus ; Caps.: Lygus, Plagiognathus.

May 10, 1910, the following 65 species and 300 individuals were taken as they came:

Long-tongued Bees $(4: 8)$-Bomb.: Bombias separatus 1, Bombus americanorum 1; Ap.: Apis 5, Nomad.: Holonomada superba 1.

Short-tongued Bees (22:189)-Prosopid.: Prosopis pygmaea 5, ziziae 1; Collet.: Colletes aestivalis 18; Halict.: Agapostemon radiatus 3 , Augochlora fervida 1, viridula 1, Chloralictus coreopsis 1, pilosus 6, tegularis 1, versatus 34, Evylaeus pectoralis 1, Halictus lerouxii 11, parellelus 1 , Odontalictus 13 , Oxystoglossa confusa 5, similis 17, Seladonia 15; Andren.: Opandrena cressonii 13, ziziae 27, Trachandrena claytoniae 1, crataegi 13, forbesii 1 .

Other Hymenoptera (14:27)-Crabron.: Clytochrysus 1, Ectemnius 1, Solenius 6, Xestocrabro texanus 1; Vesp.: Polistes variatus 2; Eumen.: Ancistrocerus tigris 2, Eumenes fraternus 1. Leionotus anormis 2 , clypeatus 2 , foraminatus 3 , histrionalis 2 , megaera 1; Tiphi.: Tiphia clypeata 1; Ichneumon.: Exyston abdominalis 2 .

Diptera (21: 69)-Bibion.: Bibio albipennis 6; Syrph.: Eristalis latifrons 1, Helophilus latifrons 2, Mesogramma marginata 4, Orthoneura pictipennis 3, Paragus bicolor 1 , tibialis 3, Sphaerophoria 4, Sphegina rufiventris 1, Syritta 1; Conop.: Zodion fulvifrons 4; Tachin.: Cistogaster occidua 5, pallasi 5, Siphona 2, Tachina, rustica 1; Sarcophag.: Helicobia helicis 1; Musc.: Lucilia caesar 1, sylvarum 5, Pseudopyrellia 1; Anthomy.: Hylemyia inornata 1 , Phorbia acra 17.

Lepidoptera (3:6)-Nymphal.: Phyciodes 1; Lycaen.: Heodes 2 ; Noctu.: Plusia simplex 3.

Hemiptera (1:1)-Pentatom.: Euschistus ictericus 1.

\section{URTICACEAe (anemophilous)}

Ulmus americana.-One visitor observed, March 26.

Long-tongued Bees (1)-Ap.: Apis c, ab.

Ulmus fulva.-One visitor observed, March 23, April 9.

Long-tongued Bees (1)-Ap.: Apis c, ab.

\section{VALERIANACEAE}

Valerianella radiata.-Blooms May 12-June 11; 24 species and 104 individuals taken on eight days, May 12-June 6 ; Mis, W. 
Long-tongued Bees (5: 1:3)-Bomb.: Tombus comsimilis \&. 1: Ceratin.: Ceratina of 7, Zaodontomerus of, 2; Shelid.: Mermetelis ơ +2 ; Megachil.: Osmia pumila $q, 1$.

Short-tongued Bees $(q, 5: 60)$ - Huliet.: Evylanus pecturals $=$ : 1, Odontalictus 1, Oxystoglossa similis 55: Prosopil.: Preopis pygmaea 1 , ziziae 2.

Other Hymenoptera (4:7)-Lumen.; Leionotus elypatan 1. illinoensis 3 , vagus 1 , Zendalia neolhuus 2.

Diptera $(9: 23)$ - Syrph.: Mesogramma marginata 5, Paramas tibialis 2, Sphaerophoria 1: Bombyli.: Tosophora 2: Tackin: Apinops 2, Cistogaster occidua 1, pallasii 8; Surcophe\%.: Ielieolia helieis 1; Anthomy.: Phorbia acra 1.

Lepidoptera (1:1)-Nymphal.: Charidryas 1.

\section{Verbinacear}

Lantana camara (eult).-Ten visitors observel. September 24-5; Mas, Y.

Birds (1) - Trochil.: Trochilus.

Long-tongued Bees (1)-Boml).: Bombus ameriennorum d.

Lepidoptera (8)-Nymphal.: Anosia; Papiliun.: Eurymus fq. Heraclides, Pieris fq, Pontia ; IIesperi.: Aneylexypha, Epareyreus: Noctu.: Plusia simplex.

Lippia lanceolata.-Blooms June 22-September 15; 56 species and 375 individuals observed, June 28-.eptember 11; Mis, W:

Long-tongued Bees (7:65)-Bomb.: Bombus amerieanorum $\varnothing$. 5; Megachil.: Megachilini : Megachile brevis of 9.32 ; Contioxyini: Coelinxys 8-dentata of 9,$3 ;$ Epeol.: Triepeolus enneolor \& 9.16 , lunatus \%, 2; Eucer.: Florilegus o f, 6, Melissodes antumnalis o. 1.

Short-tongued Bees (12:78)-Malict.: Apapostemon viridulas ㅇ, 1, Augochlora fervida $q, 4$, Chloralietus nymphasarum d. 1 , pilosus $\delta q, 3$, tegularis $q$, se, 12 , Evylaens pentoralis $q, a, 7$, Odon-

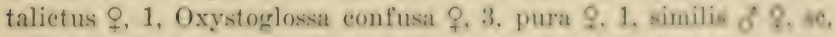
42, Seladonia 9,1 ; Panurg. : Calliopsis andreniformis $\delta 8,=2$.

Other Hymenoptera $(7: 27)$-Spher.: Priononys atrata 1. Sphex nigricans 11, pietipennis 3, vulgaris 5; Bembic: Biogrtes ventralis 4 ; Eumen.: Leionotus anormis 2, elypeatus 1.

Diptera (21: 114)-Syrph.: Eristalis latifrons 2. Helophiles latifrons 1, similis 2, Mesugramma marginata 13, Orthogeurs nitlda 1, Paragus bicolor 2, Sphacrophoria 3, Syritia 1, Tropqulim qquaimata 1; Bombyli.: Exoprosopa fasciata 7, Pascipmnis 11. Syntosehun 7: Conop.: Conops brachyrrhynehus 4; Twehin.: Apinope 1. Arehytas anil is 19. Ocyptera dosiades 1 . tenuis 7, Pachyophothaimus fluphat sis 1 , Siphoplagia anomala 6, Spallanzania 19, Triehoghore 8. 
Lepidoptera (9: 91)-Nymphal.: Phyciodes 40; Lycaen.: Chrysophanus 3, Everes 3; Papilion.: Eurema 1, Eurymus 1; Hesperi.: Ancyloxypha 21, Hylephila 1, Limochores taumas 17, Polites 5.

Verbena angustifolia.-Blooms May 22-July 18; 7 visitors observed, June 21-July 11; Mas, R.

Long-tongued Bees (4)-Ceratin.: Ceratina o ; Megachil.: Megachilini: Megachile brevis $\sigma^{\lambda} q, \mathrm{fq}$; Coelioxyini: Coelioxys 8-dentata ; Epeol.: Argyroselenis minimus $q$ type.

Short-tongued Bees (1)-Halict.: Oxystoglossa similis ô.

Other Hymenoptera (1)—Sphec.: Sphex pictipennis.

Lepidoptera (1)-Hesperi.: Pholisora catullus.

Verbena bracteosa.-Blooms June 6-September 24; 4 visitors observed, June 30, September 18; Mis, R.

Short-tongued Bees (2, ㅇ)--Halict.: Chloralictus tegularis; Panurg.: Calliopsis andreniformis.

Lepidoptera (2)-Lycaen.: Everes; Hesperi.: Hesperia.

Verbena hastata $(5: 67-8)$.-Blooms June 29-September 27; 69 visitors observed, July 12-September 18; Mas, R.

Long-tongued Bees (30)-Ap.: Apis ab; Bomb.: Bombias auricomus $\sigma^{\lambda}$, Bombus americanorum $\sigma^{\lambda} \not{\phi}, \mathrm{fq}$, impatiens $\sigma^{\lambda} \not{\phi}, \mathrm{se}$, Psithyrus laboriosus $\delta^{\top}$; Ceratin.: Ceratina $q$; Megachil.: Megachilini: Megacile brevis $\sigma^{\lambda} q$, petulans $q$, Xanthosarus $\sigma^{\lambda}$; Coelioxyini $\left(\sigma^{\lambda} q\right.$, fq) : Coelioxys 8-dentata, sayi; Epeol.: Epeolus bifasciatus ô $q$, fq, Triepeolus concavus $\delta^{\lambda}$, concolor $\delta^{\lambda} q$, fq, cressonii $\delta^{\lambda} q$, fq, helianthi

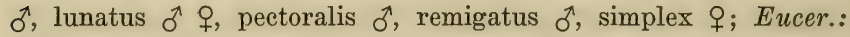
Epimelissodes atripes + , Melissodes agilis $\delta$, autumnalis $\delta$, bimaculata $\delta$, coloradensis $\delta$, comptoides $\delta$, nivea $\delta$, similima $\delta$, $\mathrm{fq}$, trinodis $\sigma^{\lambda}, \mathrm{fq}$.

Short-tongued Bees (11)-Halict.: Agapostemon radiatus $\delta$, Augochlora fervida $\delta$, Chloralictus pruinosus $\delta$, zephyrus $\delta$, Curtisapis coriacea $\delta^{\lambda}$, Halictus lerouxii $\delta \hat{\gamma}$, Odontalictus + , Oxystoglossa confusa $0^{\pi}$, ab, Seladonia $\delta^{\pi}+$, ab; Panurg.: Calliopsis andreniformis $q$, se, Verbenapis $\delta^{\top}+$, se, fq.

Other Hymenoptera (9)-Sphec.: Ammobia ichneumonea, Prionoyx atrata, Sphex gracilis $f q$, in cop, nigricans, pictipennis, procera; Bembic.: Bembix nubillipennis; Scoli.: Scolia bicincta; Vesp.: Vespula maculata.

Diptera (11)-Bombyli:: Exoprosopa fasciata ab, fascipennis, Geron rufipes, Sparnopolius, Systoechus fq, Systropus fq; Conop.: Physocephala texana fq, tibialis, Stylogaster biannulata fq; Tachin.: Archytas analis; Sarcophag.: Helicobia 4-setosa. 
Lepidoptera (6)-Papilion.: Eurymus, Pontia; Hesperi : An. cyloxypha, Epargyreus, Pholisora eatullus; Ctwnuch.: Sompin.

Coleoptera (1)-Lampyr.: Chauliognathus jumalvanious fọ. Hemiptera (1)-Caps.: Calocoris.

Verbena stricta $(5: 67)$.- Blooms June 5-Siptember $20,1: 0$ visitors observed, June 19-September 15; Mas, $R$.

Long-tongued Bees (59)-Ap.: Apis ab; Romb.; Bombias auri.

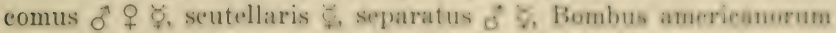

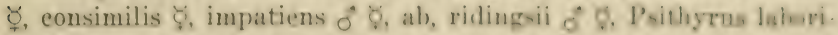

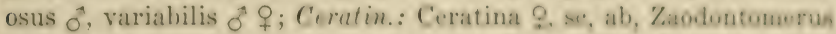
of; Stelid.: Anthidium psoraleae ob, Dianthidium of, tupe; Hege. chil.: Osmiini: Aleidamea simplex \&. se, Asloumadiella P: Mexa. ehilini $(\hat{\sigma} q)$ : Chelostomoides, Megachile brevis ah. gemoroan fo, mendiea ab, petulans ab, 6-dentata, Oligotropus ab, Sayapis mai ab, Xanthosarus fy; Coelioxyini: Coeliosys alteruata ó, fup. armuns

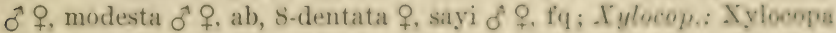

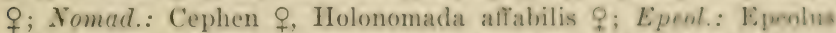
bifasciatus $\delta$, Triepeolus eoneavus of $ᄋ$, fq, roneculor $\delta$ ? , ah, ap.

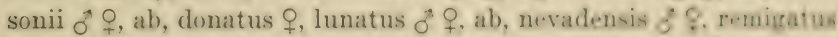

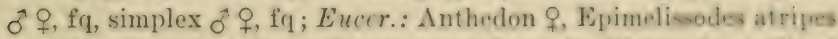

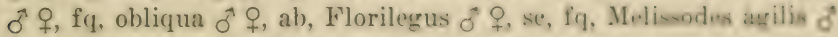
$q$, $\mathrm{f}(\mathrm{p}$, bimaculata $\hat{\sigma} \mathrm{q}$, ab, in cop, eoloradensis $\overrightarrow{0}$, comptoid. $\vec{\delta}$. $\mathrm{fq}$, coreopsis $q$, petalostemonis $q$, simillima $q$, trinodis $\delta$, variabilis

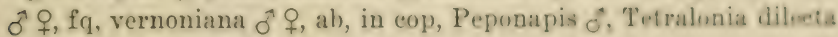
우 Emphor.: Emphor ơ.

Short-tongued Bees (11) - II alict.: Aqapostemon radiatus of ?. $\mathrm{fq}$, splendens $\hat{\delta}$, texanus of $q$, viridulus of $q$, fq. Halietus levouxii

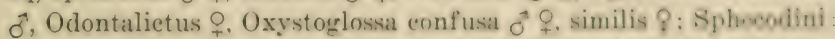
Sphecodes minor $q$; Panurg.: Calliopsis andreniformis 8 , Verhenapis $\sigma^{7}$, se, $\mathrm{fq}$, in cop.

Other Hymenoptera (5)-Sphec.: Sphex nigricans, procers, vulgaris; Bembic.: Bembix spinolae; Scoli.: Flis J-eineta.

Diptera (15) -Syrph.: Baceha elavata, Eristalis twuax: Nimer strin.: Rhynehocephalus fq; Bombyli.: Anthrax parvicornis ah, Bombylius atriceps, Exoprosopa fasciata ab. fascipennis, therun rufipes ab, Systoechus fq; Conop).: Physocephala texana, tubialis. Stylogaster biannulata $\mathrm{fq}_{\mathrm{q}}$, neglecta, Zodion fulvifrons, oblinuefos. ciatum.

Lepidoptera (30)-Nymphal.: Anosia, Argyonis, Eupteieta. Phyciodes, Vanessa huntera; Lyeuen. : Cyaniris, Evures, Urannies, Papilion.: Callidryas, Euphoeades, Eurems, Eurymons, Iphididos. Laertias, Pieris, Pontia ab; IIsperi. Anrylnsyphas. Acalopoles. 
Atrytone delaware ab, Epargyreus fq, Hesperia, Limochores taumas ab, Pholisora catullus, hayhurstii fq, Polites, Thanaos juvenalis, martialis, persius, Thorybes bathyllus; Sphing.: Hemaris axillaris.

On nine days, July 10-August 7, 1913, 58 species and 450 individuals were taken on the flowers, as follows:-

Long-tongued Bees (24:285)-Ap.: Apis 12;Bomb.: Bombias separatus 1; Ceratin.: Ceratina 25, Zaodontomerus 1; Megachil.: Megachilini : Megachile brevis 38, generosa 1, mendica 8, petulans 4, Oligotropus 2, Sapapis sayi 11; Coelioxyini: Coelioxys alternata 3, modesta 15, sayi 5; Epeol.: Triepeolus concavus 1, concolor 72, cressonii 8, lunatus 5, remigatus 1, simplex 2; Eucer.: Epimelissodes obliqua 8 , Melissodes bimaculata 32 , coloradensis 1 , comptoides 2, vernoniana 27.

Short-tongued Bees (5:12)-Halict:. Agapostemon radiatus 3, viridulus 2, Oxystoglossa confusa 3, similis 3; Panurg.: Verbenapis 1.

Other Hymenoptera (4:22)-Sphec.: Sphex nigricans 3, procera 17, vulgaris 1 ; Bembic.: Bembix spinolae 1.

Diptera (7:28)-Bombyli.: Anthrax parvicornis 1, Exoprosopa fasciata 17, fascipennis 1 , Geron rufipes 3 , Systoechus 3 ; Conop.: Stylogaster neglecta 2, Zodion fulvifrons 1.

Lepidoptera (18:103)-Nymphal.: Argynnis 1, Phyciodes 2, Vanessa huntera 2; Lycaen.: Cyaniris 1, Everes 2; Papilion.: Eurema 2, Laertias 3, Pontia 7; Hesperi.: Ancyloxypha 2, Atrytone delaware 62, Epargyreus 5, Hesperia 1, Limochores taumas 1, Pholisora hayhurstii 7, Polites 1, Thanaos martialis 1, Thorybes bathyllus 1; Sphing.: Hemaris axillaris 2.

Verbena urticaefolia (5:68).--Blooms June 19-October 5; 59 visitors observed, July 11-September 2; Mis, W.

Long-tongued Bees (13)-Ap.: Apis; Bomb.: Bombus americanorum $\sigma^{\star} \Varangle$, impatiens $\Varangle$, Ceratin.: Ceratina + , sc; Megachil.: Trypetini: Neotrypetes truncatus $\$$; Megachilini: Megachile brevis $\delta^{\lambda} q$, mendica $\delta^{\lambda} q$; Coelioxyini: Coelioxys sayi $\delta^{\lambda} q$, fq; Nomad.: Cephen $q$; Epeol: Epeolus bifasciatus $\delta$ 웅 Triepeolus concolor $\delta^{\lambda}$ , cressonii $\delta^{\top}$; Eucer.: Melissodes bimaculata ․

Short-tongued Bees (15)-Halict.: Agapostemon radiatus $\delta^{\pi}$ 우 fq, Augochlora fervida $\delta^{\lambda}+$, viridula $q$, Chloralictus pilosus $\delta^{\lambda}+$, versatus $q$, Evylaeus nelumbonis $q$, pectoralis $q$, Halictus lerouxii

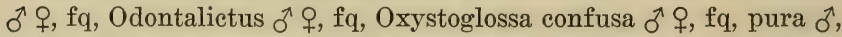

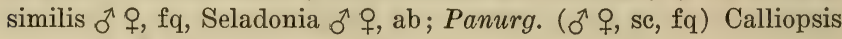
andreniformis, Verbenapis in cop. 
Other Hymenoptera (4) - Sphec.: Splex graeilis in ens, vul. garis; Scoli.: Elis 5-eineta, Seolia bieineta.

Diptera (21)-Syrph.: Baceha clavata, Mesogramma geminats. Sphaerophoria, Syritta fq; Empid.: Parempis; Bumbyli.: Anthras fulvohirta, Exoprosopa faseipenuis, Geron rufips, Systropus Toxophora; Conop.: Oneomyia, citylugaster neglesta, Zodion obliquefasciatum; Tachin.: Arehytas analis, Wistognanter costulun, Epigrimyia genieulata fy, l'horantha humeralis, siphona, spallam. zania, Trichophora fq; Musc.: Compsomyia.

Lepidoptera (6)-Nymphal.: Pligeiodes; Lyaten.: Everes fi. Uranotes; Papilion.: Eurema, Pieris, Pontia.

\section{VIOLACEAE}

Hybanthus concolor $(16: 156-7)$.- Blonms April :30-May 30 : 1 visitor observed, May $7 ; \mathrm{Mi}, \mathrm{Y}$.

Short-tongued Bees (1) - Halict.: Oxystoglosia confuka ?.

Viola cucullata (2:173).--Mlooms Mareh 17-May 21; 27 vis. itors observed, April 9-May 21; Ma, R. Inseets not reveninp an the flowers are marked $\mathrm{n}$.

Long-tongued Bees (14) $-A p$.: Apis; Bomb. (2, n): Bumbias auricomus, separatus, Bombus americanorum, impatiens: Geratin.: Ceratina ô, n; Megachil.: Centrosmia q. Crratosmin de P. Oamis

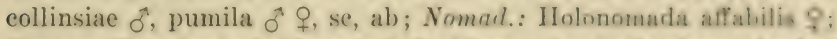
Eucer.: Tetralonia belfragei of $q$, dileeta $\delta$; Anthophor.: Antho. phora $\delta^{\top}, \mathbf{n}$.

Short-tongued Bees (5) - Mulict. ( 9 ) : Chloralietus errannii n, type, Evylaeus foxii, Seladonia n; Andren.: Andrena carlini?. Iomelissa violae $\widehat{\gamma}+$, sc, ab, type.

Diptera (2, n)-Bombyli.: Bombylius major, pulehellus.

Lepidoptera (6) - Vymphal.: Vanessa huntera: Fonitann.: Eurymus, Pieris; Hesperi.: Thanaos brizo, juvenalis, marialis.

Viola pedata $(2: 17+5)$.- Blooms April 1:-May $27: 14$ vivitor observed, April 19-May 20; Ma, R.

Long-tongued Bees (6)-Ap.: Aris: Bumb. (8): IBembia separatus, Bombus amerimanorum ab, impatiens: Tucer.: Torss. lonia dilecta ơ $0^{+}$Anthophor.: Anthophora $q$, se, ab.

Diptera (1)-Syrph.: Eristalis latifrons fsp, n.

Lepidoptera (7)-Nymphal.: Vanessa eardui : Popdion : Kury. mus, Pieris; Hesperi.: Thanaos brizo ab, irdus, juvenalie: Nowta. : Plusia simplex.

Viola pedatifida.-Blonms A pril 17-May 20: 1 visitor whemer, May 15; Ma, R. 
Long-tongued Bees (1)-Eucer.: Tetralonia dilecta $\delta^{\gamma}$.

Viola pubescens $(2: 172-3)$.-Blooms April 9-May 7; 22 visitors observed, April 16-30; Mi, Y.

Long-tongued Bees (7)-Bomb.: Bombus americanorum $q$, $\mathrm{n}$; Ceratin.: Ceratina $q$; Megachil.: Osmia atriventris + , sc, ab, pumila $\widehat{\jmath} \uparrow$, se, ab, in cop; Nomad.: Nomada sayi $q$; Eucer.: Tetralonia belfragei $q$; Anthophor.: Anthophora .

Short-tongued Bees (7, \&)-Halict.: Chloralictus pilosus sc, sparsus sc, versatus, Curtisapis coriacea, Oxystoglossa confusa se, ab, Seladonia; Andren.: Andrena carlini sc.

Diptera (5)—Syrph. (fsp, n) : Melanostoma obseurum, Mesogramma marginata, Paragus tibialis; Bombyli.: Bombylius major ab; Tachin.: Gonia capitata.

Lepidoptera (3)-Papilion.: Eurymus; Hesperi.: Thanaos juvenalis, martialis.

Viola sagittata.-Blooms April 15-May 24; 3 visitors observed, April 25-29; Ma, $\mathrm{R}$.

Long-tongued Bees (1)-Megachil.: Osmia pumila $\delta^{\lambda}, 1$.

Short-tongued Bees (1)-Andren.: Iomelissa $\sigma^{\pi}+$, sc, 4.

Lepidoptera (1)-Papilion.: Eurymus 1.

Viola striata (2:174).-Blooms April 12-June 8; 19 visitors observed, April 16-May 20; Ma, W. Visitors not reversing are marked $n$.

Long-tongued Bees (13)-Ap. Apis $\mathrm{n} ;$ Bomb. ( $(q, \mathrm{n})$ : Bombias auricomus, separatus, Bombus americanorum, consimilis, impatiens; Ceratin.: Zaodontomerus on n ; Megachil. (sc) : Diceratosmia $q$, Osmia atriventris $q$, collinsiae $\delta \uparrow$, fq, pumila $q$, fq ; Eucer.: Tetralonia belfragei $\sigma^{\top}+, f q, n$, dilecta $\delta, n$.

Short-tongued Bees (3, q)-Halict.: Curtisapis coriacea; $A n$ dren.: Andrena carlini, Iomelissa se, fq.

Diptera (1)-Bombyli: Bombylius major $\mathrm{n}$.

Lepidoptera (2, n)-Papilion.: Eurymus; Hesperi.: Thanaos persius.

\section{VitACEAE}

Psedera quinquefolia.-Blooms June 13-July 27; 1 visitor observed, June 22, July 27; Mas, Y.

Long-tongued Bees (1)-Megachil.: Megachile-mendica $\delta^{\lambda}$, sc, ab.

Vitis cordifolia.-Blooms June 5-22; 3 visitors observed, June 15; Mas, $\mathrm{Y}$. 


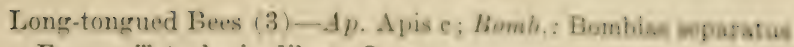
o, e; Eucer.: 'T'etralonia dilecta ㅇ․

Vitis vulpina.-Blooms May 12-June 1; 3 vivion oteornot. May 16-29; Mas, $Y$.

Long-tongued Boes (3. ‥ c)-Bomh.: Pombias wparatus, Bhom bus americanorum, impatiens.

\section{Flowers axd Ixsects Paprens Citen}

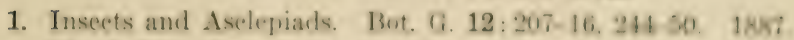

2. Fls. I-III. Bot. G. 14:120-6, 172-8, 297-304. 1859.

3. Fls. IV, V. Bot. G. 15: 79-84, 199-204. 1890.

4. Fls. VI. Bot. G. $16: 65-71.1891$.

5. Fls. VII-IX. Bot. G. 17:65-71, 173-9, 269-76. 1892.

6. Fls. X, XI. Bot. G. 18:47-54, 267-74. 1893.

7. Fls. XII. Bot. G. 19:103-12. 1894.

8. Fls. XIII, XIV. Bot. G. $20: 104-10,139-49.1895$.

9. Fls. XT-XVII. Bot. F. $21: 72-81.266-74: 22: 164-65,1596$. 10. Fls. XVIII. Bot. G. $25: 229-45.1898$.

11. Fls. XIX. Bot. G. $28: 27-45.1899$.

12. Fls. Umbelliferae. St. L. A. 5:449-60, 1890.

13. Fls. Aselep. to Seroph. St. L. A. 5:569-98. 1891.

14. Fls. Labiatae. St. L. A. 6:101-31. 1892.

15. Fls. Rosaceae and Compositae. St. I. A. 6:435-80. 1 ke4.

16. Fls. Contributions. St. I. A. 7:151-79. 1896. 






UNIVERSITY OF ILLINOIS-URBANA 